\title{
Neutrino masses and mixing: evidence and implications
}

\author{
M. C. Gonzalez-Garcia \\ Theory Division, CERN, CH-1211, Geneva 23, Switzerland; \\ Instituto de Física Corpuscular, Universitat de València-C.S.I.C, Edificio Institutos de \\ Paterna, Apt. 22085, 46071 València, Spain; \\ C.N. Yang Institute for Theoretical Physics, State University of New York at Stony Brook, \\ Stony Brook, New York 11794-3840 \\ Yosef Nir \\ Department of Particle Physics, Weizmann Institute of Science, Rehovot 76100, Israel
}

(Published 26 March 2003)

\begin{abstract}
Measurements of various features of the fluxes of atmospheric and solar neutrinos have provided evidence for neutrino oscillations and therefore for neutrino masses and mixing. The authors review the phenomenology of neutrino oscillations in vacuum and in matter. They present the existing evidence from solar and atmospheric neutrinos as well as the results from laboratory searches, including the final status of the Liquid Scintillator Neutrino Detector (LSND) experiment. The theoretical inputs that are used to interpret the experimental results are described in terms of neutrino oscillations. The allowed ranges for the mass and mixing parameters are derived in two frameworks: First, each set of observations is analyzed separately in a two-neutrino framework; Second, the data from solar and atmospheric neutrinos are analyzed in a three-active-neutrino framework. The theoretical implications of these results are then discussed, including the existence of new physics, the estimate of the scale of this new physics, and the lessons for grand unified theories, for models of extra dimensions and singlet fermions in the bulk, and for flavor models.
\end{abstract}

\section{CONTENTS}

I. Introduction

D. Lepton mixing

III. Neutrino Oscillations

IV. Solar Neutrinos

II. The Standard Model and Neutrino Masses

A. The standard model implies $m_{\nu}=0$

B. Extensions of the standard model allow $m_{\nu} \neq 0$

C. Dirac and Majorana neutrino mass terms

A. Neutrino oscillations in vacuum

B. Neutrinos in matter: Effective potentials

C. Evolution equation in matter: Effective mass and mixing

D. Adiabatic versus nonadiabatic transitions

E. Propagation in the sun: Mikheyev-SmirnovWolfenstein effect

A. Solar neutrino experiments

1. Chlorine experiment: Homestake

2. Gallium experiments: SAGE and GALLEX/GNO

3. Water Cerenkov experiments: Kamiokande and SuperKamiokande

4. SNO

5. Future: Borexino and low-energy experiments

B. The solar neutrino problem

C. Solar neutrino oscillation probabilities

1. Quasivacuum oscillations and the dark side

2. Evolution in the Earth

D. Two-neutrino oscillation analysis

1. Predictions

2. Analysis of total event rates: allowed masses and mixing

346

347

348

348

349

350

351

351

352

354

355

357

358

360

360

360

360

361

362

363

364

364

365

366

366

367

3. Day-night spectra: Excluded masses and mixing
4. Global analysis

368

V. Atmospheric Neutrinos 369

A. Atmospheric neutrino fluxes 371

1. Cosmic-ray spectrum 372

2. Geomagnetic effects 372

3. The neutrino yield 373

4. The neutrino fluxes 373

B. Interaction cross sections 374

C. Two-neutrino oscillation analysis $\quad 375$

1. Predicted number of events 375

2. Conversion probabilities 376

3. Statistical analysis 376

4. $\nu_{\mu} \rightarrow \nu_{e} \quad 377$

5. $\nu_{\mu} \rightarrow \nu_{\tau}$ and $\nu_{\mu} \rightarrow \nu_{s}$

VI. Laboratory Experiments 379

A. Short-baseline experiments at accelerators 379

B. LSND and KARMEN 379

C. Disappearance experiments at reactors 381

D. Long-baseline experiments at accelerators 382

E. Direct determination of neutrino masses 382

VII. Three-Neutrino Mixing 383

A. Probabilities 384

B. Allowed masses and mixing 386

VIII. Implications of the Neutrino Mass Scale and Flavor Structure $\quad 387$

A. New physics $\quad 387$

B. The scale of new physics 388

C. Implications for flavor physics 388

1. The flavor parameters 388

2. Special features of the neutrino flavor 389

3. Large mixing and strong hierarchy 390

IX. Implications for Models of New Physics 393

A. Grand unified theories 393

B. Extra dimensions 393

1. Coupling to bulk fermions 394 


$\begin{array}{ll}\text { 2. Lepton number breaking on a distant brane } & 395 \\ \text { 3. The warp factor in the Randall-Sundrum } & \\ \quad \text { scenario } & 395 \\ \text { X. Conclusions } & 395 \\ \text { Note added } & 396 \\ \text { KamLAND results } & 396 \\ \text { Consequences for solar neutrinos } & 397 \\ \text { Effect on three-neutrino mixing scenarios } & 398 \\ \text { Acknowledgments } & 398 \\ \text { References } & 398\end{array}$

\section{INTRODUCTION}

In 1930 Wolfgang Pauli postulated the existence of the neutrino in order to reconcile data on the radioactive decay of nuclei with energy conservation. In radioactive decays, nuclei of atoms mutate into different nuclei when neutrons are transformed into slightly lighter protons with the emission of electrons:

$$
\text { neutron } \rightarrow \text { proton }+ \text { electron }+ \text { antineutrino. }
$$

Without the neutrino, energy conservation would require that the electron and proton share the neutron's energy. Each electron would therefore be produced with a fixed energy, while experiments indicated conclusively that electrons were not monoenergetic but were observed with a range of energies. This energy range corresponded exactly to the many ways the three particles in the final state of the reaction above could share energy while satisfying the conservation law. The postulated neutrino had no electric charge and, for all practical purposes, did not interact with matter; it just served as an agent to balance energy and momentum in the above reaction. In fact, Pauli pointed out that for the neutrino to do the job, it had to weigh less than one percent of the proton mass, thus establishing the first limit on neutrino mass.

Observing neutrinos is straightforward-in principle. Pauli had to wait a quarter of a century before Fred Reines and Clyde Cowan, Jr. observed neutrinos produced by a nuclear reactor. In the presence of protons, neutrinos occasionally initiate the inverse reaction of radioactive decay:

$$
\bar{\nu}+p \rightarrow n+e^{+} .
$$

Experimentally, one exposes a material rich in protons to a neutrino beam and simply looks for the coincident appearance of an electron and a neutron. In the alternative possibility where the incident neutrino carries muon flavor, a muon appears in the final state instead.

By the 1960s neutrino beams were no longer a futuristic dream, but had become one of the most important tools of particle physics. Neutrino physics contributed in important ways to the discovery of quarks, the constituents of protons and neutrons. The technique is conceptually simple, though technologically very challenging. A very intense beam of accelerated protons is shot into a beam dump which typically consists of a kilometer-long mound of earth or a 100-meter-long block of stainless steel. Protons interact with nuclei in the dump and produce tens of pions in each collision. Charged pions decay into a muon and a neutrino. The material in the dump will eventually absorb the muons, photons, and any other charged particles, so that only neutrinos exit at the opposite end, forming an intense and controlled beam.

Neutrinos are also produced in natural sources. From the 1960s on, neutrinos produced in the sun and in the atmosphere have been observed. As we shall see in this review, these observations play an important role in expanding our understanding of the detailed features of neutrinos. In 1987, neutrinos from a supernova in the Large Magellanic Cloud were also detected.

The properties of the neutrino and in particular the question of its mass have intrigued physicists ever since it was proposed (Kayser, Gibrat-Debu, and Perrier, 1989; Ramond, 1999). In the laboratory neutrino masses have been searched for in two types of experiments (Boehm and Vogel, 1987): (i) direct kinematic searches of neutrino mass, of which the most sensitive is the study of tritium beta decay, and (ii) neutrinoless double- $\beta$ decay experiments. Experiments have achieved higher and higher precision, reaching upper limits for the electron neutrino mass of $10^{-9}$ of the proton mass, rather than the $10^{-2}$ originally obtained by Pauli. This raised the question of whether neutrinos are truly massless like photons.

Can one go further below the $\mathrm{eV}$ scale (that is, $10^{-9}$ of the proton mass) in the search for neutrino masses? This is a very difficult task in direct measurements. In 1957, however, Bruno Pontecorvo realized that the existence of neutrino masses implied the possibility of neutrino oscillations. This phenomenon is similar to what happens in the quark sector, where neutral kaons oscillate. Flavor oscillations of neutrinos have been searched for using either neutrino beams from reactors or accelerators, or natural neutrinos generated at astrophysical sources (the Sun giving the largest flux) or in the atmosphere (as the by-products of cosmic-ray collisions). The longer the distance that the neutrinos travel from their production point to the detector, the smaller the masses that can be signalled by their oscillation. Indeed, the solar neutrinos allow us to search for masses that are as small as $10^{-5} \mathrm{eV}$, that is, $10^{-14}$ of the proton mass!

In recent years, experiments studying natural neutrino fluxes have provided us with the strongest evidence of neutrino masses and mixing. Experiments that measure the flux of atmospheric neutrinos have found results that suggest the disappearance of muon-neutrinos when propagating over distances of the order of hundreds of kilometers (or more). Experiments that measure the flux of solar neutrinos (Bahcall, 1989) suggest the disappearance of electron neutrinos while propagating within the Sun or between the Sun and the Earth. The disappearance of both atmospheric $\nu_{\mu}$ 's and solar $\nu_{e}$ 's is most easily explained in terms of neutrino oscillations. As concerns experiments performed with laboratory beams, most have given no evidence of oscillations. One exception is the Liquid Scintillator Neutrino Detector (LSND) experiment, which has observed the appear- 
ance of electron antineutrinos in a muon antineutrino beam. This signal has not been confirmed so far by any other experiment.

What can we learn from measurements of neutrino masses about our theories of particle physics? The standard model of particle physics is a mathematical description of the strong, weak, and electromagnetic interactions. Since it was conceived in the 1960 s by Glashow, Salam, and Weinberg, it has successfully passed numerous experimental tests. In the absence of any direct evidence for their mass, neutrinos were introduced in the standard model as truly massless fermions for which no gauge-invariant renormalizable mass term could be constructed. Consequently, in the standard model there is neither mixing nor $C P$ violation in the lepton sector. Therefore experimental evidence for neutrino masses or mixing or leptonic $C P$ violation provides an unambiguous signal of new physics.

The standard-model prediction of massless neutrinos is accidental: unlike photons, no profound principle prevents them from having a mass. On the contrary, modern elementary particle theories anticipate ways in which they might have small but definitely nonvanishing masses. In fact, there are good theoretical reasons to expect that neutrinos are massive but much lighter than all the charged fermions of the standard model. Specifically, it is very likely that neutrino masses are inversely proportional to the scale of new physics. Consequently, if neutrino masses are measured, we can estimate the relevant new scale.

All dimensionless flavor-blind parameters of the standard model, that is, the three gauge couplings and the quartic Higgs coupling, are of order one. In contrast, most of the flavor parameters-quark and chargedlepton masses (except for the mass of the top quark), and the three Cabibbo-Kobayashi-Maskawa (CKM) mixing angles-are small and hierarchical. This situation constitutes the flavor puzzle: Within the standard model, the hierarchical structure can be accommodated but is not explained. If neutrinos have Majorana (Dirac) masses, then there are nine (seven) flavor parameters beyond the thirteen of the standard model. If some of these extra parameters are measured, we will be able to test and refine theories that try to solve the flavor puzzle.

In grand unified theories (GUT's), lepton masses are often related to quark masses. Measurements of neutrino parameters provide further tests of these theories.

The values of neutrino parameters that explain the anomalies observed in atmospheric and solar neutrino fluxes can be used to address the many theoretical questions described above: they imply new physics, they suggest an energy scale at which this new physics takes place, they provide stringent tests of flavor models, and they probe GUT's.

In this review we first present the low-energy formalism for adding neutrino masses to the standard model and the induced leptonic mixing (Sec. II) and then describe the phenomenology associated with neutrino oscillations in vacuum and in matter (Sec. III). In Secs. IV and $\mathrm{V}$ we discuss the evidence from solar and atmospheric neutrinos (for further details we refer the reader to the recent review on atmospheric neutrino observations by Kajita and Totsuka, 2001). We review the theoretical modeling that is involved in interpreting the experimental results in terms of neutrino oscillations and briefly describe the techniques used in the derivation of the allowed ranges for the neutrino flavor parameters which, in these sections, is performed in the framework of mixing between two neutrinos. Section VI is devoted to the results from searches in laboratory experiments concentrating on the final status of the LSND experiment (a detailed review of the status of neutrino oscillation searches employing nuclear reactors as sources can be found in Bemporad, Gratta, and Vogel, 2002). The two most robust pieces of evidence, from solar and atmospheric neutrinos, can be accommodated assuming masses and mixing of three standard neutrinos: in Sec. VII we derive the allowed ranges of parameters in this case. In Secs. VIII and IX we discuss the various implications for theory based on this evidence, focusing on models that explain the atmospheric and solar neutrino data through mixing among three active neutrinos. In particular, we discuss flavor models, GUTs, and models of extra dimensions. Our conclusions are summarized in Sec. X.

\section{THE STANDARD MODEL AND NEUTRINO MASSES}

One of the most beautiful aspects of modern theories of particles physics is the relation between forces mediated by spin-1 particles and local (gauge) symmetries. Within the standard model, the strong, weak, and electromagnetic interactions are related to, respectively, $\mathrm{SU}(3), \mathrm{SU}(2)$, and $\mathrm{U}(1)$ gauge groups. Many features of the various interactions are then explained by the symmetry to which they are related. In particular, the way that the various fermions are affected by the different types of interactions is determined by their representations (or simply their charges in the case of Abelian gauge symmetries) under the corresponding symmetry groups.

Neutrinos are fermions that have neither strong nor electromagnetic interactions. In group theory language, they are singlets of $\mathrm{SU}(3)_{\mathrm{C}} \times \mathrm{U}(1)_{\mathrm{EM}}$. Active neutrinos have weak interactions, that is, they are not singlets of $\mathrm{SU}(2)_{\mathrm{L}}$. Sterile neutrinos have none of the standard model gauge interactions and they are singlets of the standard model gauge group.

The standard model has three active neutrinos. They reside in lepton doublets,

$$
L_{\ell}=\left(\begin{array}{c}
\nu_{L \ell} \\
\ell_{L}^{-}
\end{array}\right), \quad \ell=e, \mu, \tau .
$$

Here $e, \mu$, and $\tau$ are the charged-lepton mass eigenstates. The three neutrino interaction eigenstates, $\nu_{e}$, $\nu_{\mu}$, and $\nu_{\tau}$, are defined as the $\mathrm{SU}(2)_{\mathrm{L}}$ partners of these mass eigenstates. In other words, the charged-current interaction terms for leptons read 


$$
-\mathcal{L}_{\mathrm{CC}}=\frac{g}{\sqrt{2}} \sum_{\ell} \overline{\nu_{L \ell}} \gamma^{\mu} \ell_{L}^{-} W_{\mu}^{+}+\text {H.c. }
$$

In addition, standard model neutrinos have neutralcurrent interactions,

$$
-\mathcal{L}_{\mathrm{NC}}=\frac{g}{2 \cos \theta_{W}} \sum_{\ell} \overline{\nu_{L \ell}} \gamma^{\mu} \nu_{L \ell} Z_{\mu}^{0} .
$$

Equations (4) and (5) give all the neutrino interactions within the standard model.

The measurement of the decay width of the $Z^{0}$ boson into neutrinos makes the existence of three, and only three, light (that is, $m_{\nu} \leqslant m_{Z} / 2$ ) active neutrinos an experimental fact. In units of the standard model prediction for a single neutrino, one gets (Groom et al., 2000)

$$
N_{\nu}=2.994 \pm 0.012
$$

(standard model fits to LEP data),

$$
N_{\nu}=3.00 \pm 0.06
$$

(direct measurement of invisible $Z$ width).

\section{A. The standard model implies $m_{\nu}=0$}

The standard model is based on the gauge group

$$
G_{\mathrm{SM}}=\mathrm{SU}(3)_{\mathrm{C}} \times \mathrm{SU}(2)_{\mathrm{L}} \times \mathrm{U}(1)_{\mathrm{Y}},
$$

with three fermion generations, where a single generation consists of five different representations of the gauge group,

$$
\begin{array}{ll}
Q_{L}(3,2)_{+1 / 6}, & U_{R}(3,1)_{+2 / 3}, D_{R}(3,1)_{-1 / 3}, \\
L_{L}(1,2)_{-1 / 2}, & E_{R}(1,1)_{-1} .
\end{array}
$$

Our notation here means that, for example, a lefthanded lepton field $L_{L}$ is a singlet (1) of the $\mathrm{SU}(3)_{\mathrm{C}}$ group, a doublet (2) of the $\mathrm{SU}(2)_{\mathrm{L}}$ group, and carries hypercharge $-1 / 2$ under the $U(1)_{Y}$ group.

The vacuum expectations value of the single Higgs doublet $\phi(1,2)_{+1 / 2}$ breaks the symmetry,

$$
\langle\phi\rangle=\left(\begin{array}{c}
0 \\
\frac{v}{\sqrt{2}}
\end{array}\right) \Rightarrow G_{\mathrm{SM}} \rightarrow \mathrm{SU}(3)_{\mathrm{C}} \times \mathrm{U}(1)_{\mathrm{EM}} .
$$

Since the fermions reside in chiral representations of the gauge group, there can be no bare mass terms. Fermions masses arise from the Yukawa interactions,

$$
\begin{aligned}
-\mathcal{L}_{\text {Yukawa }}= & Y_{i j}^{d} \overline{Q_{L i}} \phi D_{R j}+Y_{i j}^{u} \overline{Q_{L i}} \tilde{\phi} U_{R j} \\
& +Y_{i j}^{\ell} \overline{L_{L i}} \phi E_{R j}+\text { H.c. }
\end{aligned}
$$

(where $\widetilde{\phi}=i \tau_{2} \phi^{\star}$ ) after spontaneous symmetry breaking. The Yukawa interactions of Eq. (10) lead to charged fermion masses but leave the neutrinos massless. One might think that neutrino masses would arise from loop corrections if these corrections induced effective terms,

$$
\frac{Y_{i j}^{v}}{v} \phi \phi L_{L i} L_{L j}
$$

This, however, cannot happen, as can be easily understood by examining the accidental symmetries of the standard model. (It often happens that, as a consequence of the symmetries that define a model and of its particle content, all renormalizable Lagrangian terms obey additional symmetries, which are not a priori imposed on the model. These are called accidental symmetries.) Within the standard model, with the gauge symmetry of Eq. (7) and the particle content of Eq. (8), the following accidental global symmetry arises (at the perturbative level):

$$
G_{\mathrm{SM}}^{\text {global }}=\mathrm{U}(1)_{B} \times \mathrm{U}(1)_{e} \times \mathrm{U}(1)_{\mu} \times \mathrm{U}(1)_{\tau} .
$$

Here $\mathrm{U}(1)_{B}$ is the baryon number symmetry, and $\mathrm{U}(1)_{e, \mu, \tau}$ are the three lepton flavor symmetries, with total lepton number given by $L=L_{e}+L_{\mu}+L_{\tau}$. Terms of the form of Eq. (11) violate $G_{\mathrm{SM}}^{\text {global }}$ and therefore cannot be induced by loop corrections. Furthermore, the $\mathrm{U}(1)_{B-L}$ subgroup of $G_{\mathrm{SM}}^{\text {global }}$ is nonanomalous. Terms of the form of Eq. (11) have $B-L=-2$ and therefore cannot be induced even by nonperturbative corrections.

It follows that the standard model predicts that neutrinos are precisely massless. Consequently, there is neither mixing nor $C P$ violation in the leptonic sector.

\section{B. Extensions of the standard model allow $m_{\nu} \neq 0$}

There are many good reasons to think that the standard model is not a complete picture of Nature. For example, the fine-tuning problem of the Higgs mass can be solved by supersymmetry; gauge coupling unification and the variety of gauge representations may find an explanation in GUT's; baryogenesis can be initiated by decays of heavy singlet fermions (leptogenesis); and the existence of gravity suggests that string theories are relevant to Nature. If any of these (or many other proposed) extensions is indeed realized in nature, the standard model must be thought of as an effective lowenergy theory. That means that it is a valid approximation up to the scale $\Lambda_{\mathrm{NP}}$ which characterizes new physics.

By thinking of the standard model as an effective lowenergy theory, we still retain the gauge group (7), the fermionic spectrum (8), and the pattern of spontaneous symmetry breaking [Eq. (9)] as valid ingredients to describe nature at energies $E \ll \Lambda_{\mathrm{NP}}$. The standard model predictions are, however, modified by small effects that are proportional to powers of $E / \Lambda_{\mathrm{NP}}$. In other words, the difference between the standard model as a complete description of Nature and as a low-energy effective theory is that, in the latter case, we must also consider nonrenormalizable terms.

There is no reason for generic new physics to respect the accidental symmetries of Eq. (12). Indeed, there is a single set of dimension-five terms that is made of standard-model fields and is consistent with gauge symmetry, and this set violates Eq. (12). It is given by 


$$
\frac{Z_{i j}^{\nu}}{\Lambda_{\mathrm{NP}}} \phi \phi L_{L i} L_{L j}
$$

While these terms have the same form as Eq. (11) they do not have radiative corrections as their source, but rather some heavy fields, related to new physics, which could induce such terms by tree or loop diagrams. In particular, Eq. (13) violates $L$ (and $B-L$ ) by two units and leads, upon spontaneous symmetry breaking, to neutrino masses:

$$
\left(M_{\nu}\right)_{i j}=\frac{Z_{i j}^{\nu}}{2} \frac{v^{2}}{\Lambda_{\mathrm{NP}}} .
$$

A few comments are in order, regarding Eq. (14):

(a) Since Eq. (14) would arise in a generic extension of the standard model, we learn that neutrino masses are very likely to appear if there is new physics.

(b) If neutrino masses arise effectively from nonrenormalizable terms, we gain an explanation not only for their existence but also for their smallness. The scale of neutrino masses is suppressed, compared to the scale of charged fermion masses, by $v / \Lambda_{\mathrm{NP}}$.

(c) The terms of Eq. (14) break not only total lepton number but also the lepton flavor symmetry $\mathrm{U}(1)_{e} \times \mathrm{U}(1)_{\mu} \times \mathrm{U}(1)_{\tau}$. Therefore we should expect lepton mixing and $C P$ violation.

The best known scenario that leads to Eq. (13) is the seesaw mechanism (Gell-Mann et al., 1979; Ramond, 1979; Yanagida, 1979). Here one assumes the existence of heavy sterile neutrinos $N_{i}$. Such fermions have, in general, bare mass terms and Yukawa interactions:

$$
-\mathcal{L}_{N}=\frac{1}{2} M_{N i j} \overline{N_{i}^{c}} N_{j}+Y_{i j}^{\nu} \overline{L_{L i}} \widetilde{\phi} N_{j}+\text { H.c. }
$$

The resulting mass matrix (see Sec. II.C for details) in the basis

$$
\left(\begin{array}{c}
\nu_{L i} \\
N_{j}
\end{array}\right)
$$

has the following form:

$$
M_{\nu}=\left(\begin{array}{cc}
0 & Y^{\nu} \frac{v}{\sqrt{2}} \\
\left(Y^{\nu}\right)^{T} \frac{v}{\sqrt{2}} & M_{N}
\end{array}\right) .
$$

If the eigenvalues of $M_{N}$ are all well above the electroweak breaking scale $v$, then the diagonalization of $M_{\nu}$ leads to three light mass eigenstates with a mass matrix of the form of Eq. (14). In particular, the scale $\Lambda_{\mathrm{NP}}$ is identified with the mass scale of the heavy sterile neutrinos, that is, the typical scale of the eigenvalues of $M_{N}$.

Two well-known examples of extensions of the standard model that lead to a seesaw mechanism for neutrino masses are SO(10) GUT's (Gell-Mann et al., 1979;
Ramond, 1979; Yanagida, 1979) and left-right symmetry (Mohapatra and Senjanovic, 1980).

\section{Dirac and Majorana neutrino mass terms}

If the only modification that we make to the standard model is to assume that it is a low-energy effective theory, that is, allowing for nonrenormalizable terms that are consistent with gauge symmetry and the fermionic content of the standard model, then the only way that neutrinos can gain masses is through terms of the form of Eq. (13). These are Majorana mass terms which, in particular, violate lepton number by two units.

One can, however, open up other possibilities by adding new fields. The most relevant extension is to add an arbitrary number $m$ of sterile neutrinos $\nu_{s i}(1,1)_{0}$ to the three standard generations of Eq. (8). Now there are, in general, two types of mass terms that arise from renormalizable terms:

$$
-L_{M_{\nu}}=M_{D i j} \overline{\nu_{L i}} \nu_{s j}+\frac{1}{2} M_{N i j} \overline{\nu_{s i}^{c}} \nu_{s j}+\text { H.c. }
$$

Here $\nu^{c}$ indicates a charge-conjugated field, $\nu^{c}=C \bar{\nu}^{T}$, and $C$ is the charge conjugation matrix.

The first term is a Dirac mass term. It has the following properties:

(i) Since it transforms as the doublet representation of $\mathrm{SU}(2)_{\mathrm{L}}$, it is generated after spontaneous electroweak symmetry breaking from the Yukawa interactions $Y_{i j}^{\nu} \overline{L_{L i}} \widetilde{\phi} \nu_{s j}$, similarly to the charged fermion masses discussed in Sec. II.A.

(ii) Since it has a neutrino field and an antineutrino field, it conserves total lepton number [though it breaks the lepton flavor number symmetries of Eq. (12)].

(iii) $M_{D}$ is a complex $3 \times m$ matrix.

The second term in Eq. (17) is a Majorana mass term. It is different from the Dirac mass terms in many important aspects:

(i) It is a singlet of the standard model gauge group. Therefore it can appear as a bare mass term. [Had we written a similar term for the active neutrinos, it would transform as a triplet of $\mathrm{SU}(2)_{\mathrm{L}}$. In the absence of a Higgs triplet, it cannot be generated by renormalizable Yukawa interactions. Such terms are generated for active neutrinos from the nonrenormalizable Yukawa interactions of Eq. (13).]

(ii) Since it involves two neutrino fields, it breaks lepton number conservation by two units. More generally, such a term is allowed only if the neutrinos carry no additive conserved charge. This is the reason that such terms are not allowed for any charged fermions which, by definition, carry $\mathrm{U}(1)_{\mathrm{EM}}$ charges.

(iii) $\quad M_{N}$ is a symmetric matrix (as follows from simple Dirac algebra) of dimension $m \times m$. 
It is convenient to define a $(3+m)$-dimensional neutrino vector $\vec{\nu}$,

$$
\vec{\nu}=\left(\begin{array}{c}
\nu_{L i} \\
\nu_{s j}
\end{array}\right) .
$$

That allows us to rewrite Eq. (17) in a unified way:

$$
-L_{M_{\nu}}=\frac{1}{2} \overline{\vec{\nu}^{c}} M_{\nu} \vec{\nu}+\text { H.c. }
$$

where

$$
M_{\nu}=\left(\begin{array}{cc}
0 & M_{D} \\
M_{D}^{T} & M_{N}
\end{array}\right) .
$$

The matrix $M_{\nu}$ is complex and symmetric. It can be diagonalized by a unitary matrix of dimension $(3+m)$. The resulting mass eigenstates, $\nu_{k}$, obey the Majorana condition, $\nu_{k}^{c}=\nu_{k}$.

There are three interesting cases, differing in the hierarchy of scales between $M_{N}$ and $M_{D}$ :

(1) One can assume that the scale of the mass eigenvalues of $M_{N}$ is much higher than the scale of electroweak symmetry breaking $\langle\phi\rangle$. This is the natural situation in various extensions of the standard model that are characterized by a high energy scale. We are back in the framework of the seesaw mechanism discussed in the previous section. If we simply integrate out the sterile neutrinos, we get a low-energy effective theory with three light, active neutrinos of the Majorana type.

(2) One can assume that the scale of some eigenvalues of $M_{N}$ is not higher than the electroweak scale. Now the standard model is not even a good low-energy effective theory: there are more than three light neutrinos, and they are mixtures of doublet and singlet fields. These light fields are all of the Majorana type.

(3) One can assume that $M_{N}=0$. This is equivalent to imposing lepton number symmetry on this model. Again, the standard model is not even a good lowenergy theory: both the fermionic content and the assumed symmetries are different. (Recall that within the standard model lepton number is an accidental symmetry.) Now only the first term in Eq. (17) is allowed, which is a Dirac mass term. It is generated by the Higgs mechanism in the same way that charged fermions masses are generated. If indeed it is the only neutrino mass term present and $m=3$, we can identify the three sterile neutrinos with the right-hand component of a four-component spinor neutrino field (actually with its charge conjugate). In this way, the six massive Majorana neutrinos combine to form three massive neutrino Dirac states, equivalent to the charged fermions. In this particular case the $6 \times 6$ diagonalizing matrix is block diagonal and it can be written in terms of a $3 \times 3$ unitary matrix.

From the phenomenological point of view, it will make little difference for our purposes whether the light neutrinos are of the Majorana or Dirac type. In particular, the analysis of neutrino oscillations is the same in both cases. Only in the discussion of neutrinoless double beta decay will the question of Majorana versus Dirac neutrinos be crucial.

From the theoretical model-building point of view, however, the two cases are very different. In particular, the seesaw mechanism provides a natural explanation for the lightness of Majorana neutrinos, while for Dirac neutrinos there is no such generic mechanism. (The situation can be different in models of extra dimensions; see Sec. IX.B.)

\section{Lepton mixing}

We briefly review here our notation for lepton mixing. We denote the neutrino mass eigenstates by $\left(\nu_{1}, \nu_{2}, \nu_{3}, \ldots, \nu_{n}\right)$ where $n=3+m$, and the chargedlepton mass eigenstates by $(e, \mu, \tau)$. The corresponding interaction eigenstates are denoted by $\left(e^{I}, \mu^{I}, \tau^{I}\right)$ and $\vec{\nu}$ $=\left(\nu_{L e}, \nu_{L \mu}, \nu_{L \tau}, \nu_{s 1}, \ldots, \nu_{s m}\right)$. In the mass basis, leptonic charged-current interactions are given by

$$
-\mathcal{L}_{\mathrm{CC}}=\frac{g}{\sqrt{2}}\left(\overline{e_{L}} \overline{\mu_{L}} \overline{\tau_{L}}\right) \gamma^{\mu} U\left(\begin{array}{c}
\nu_{1} \\
\nu_{2} \\
\nu_{3} \\
\vdots \\
\nu_{n}
\end{array}\right) W_{\mu}^{+}-\text {H.c. }
$$

Here $U$ is a $3 \times n$ matrix (Schechter and Valle, 1980a, 1980b).

Given the charged-lepton mass matrix $M_{\ell}$ and the neutrino mass matrix $M_{\nu}$ in some interaction basis,

$$
-\mathcal{L}_{M}=\left(\overline{e_{L}^{I}} \overline{\mu_{L}^{I}} \overline{\tau_{L}^{I}}\right) \quad M_{\ell}\left(\begin{array}{c}
e_{R}^{I} \\
\mu_{R}^{I} \\
\tau_{R}^{I}
\end{array}\right)+\frac{1}{2} \overline{\vec{\nu}^{c}} M_{\nu} \vec{\nu}+\text { H.c. }
$$

we can find the diagonalizing matrices $V^{\ell}$ and $V^{\nu}$ :

$$
\begin{aligned}
& V^{\ell \dagger} M_{\ell} M_{\ell}^{\dagger} V^{\ell}=\operatorname{diag}\left(m_{e}^{2}, m_{\mu}^{2}, m_{\tau}^{2}\right), \\
& V^{\nu^{\dagger}} M_{\nu}^{\dagger} M_{\nu} V^{\nu}=\operatorname{diag}\left(m_{1}^{2}, m_{2}^{2}, m_{3}^{2}, \ldots, m_{n}^{2}\right) .
\end{aligned}
$$

Here $V^{\ell}$ is a unitary $3 \times 3$ matrix while $V^{\nu}$ is a unitary $n \times n$ matrix. The $3 \times n$ mixing matrix $U$ can be found from these diagonalizing matrices:

$$
U_{i j}=P_{\ell, i i} V_{i k}^{\ell \dagger} V_{k j}^{\nu}\left(P_{\nu, j j}\right) \text {. }
$$

A few comments are in order:

(i) Note that the indices $i$ and $k$ run from 1 to 3, while $j$ runs from 1 to $n$. In particular, only the first three lines of $V^{\nu}$ play a role in Eq. (24).

(ii) $\quad P_{\ell}$ is a diagonal $3 \times 3$ phase matrix that is conventionally used to reduce by three the number of phases in $U$.

(iii) $P_{\nu}$ is a diagonal matrix with additional arbitrary phases (chosen to reduce the number of phases in $U$ ) only for Dirac states. For Majorana neutrinos, this matrix is simply a unit matrix. The reason for that is that if one rotates a Majorana neutrino by a phase, this phase will appear in its mass term, which will no longer be real. 
We conclude that the number of phases that can be absorbed by redefining the mass eigenstates depends on whether the neutrinos are Dirac or Majorana particles. In particular, if there are only three Majorana neutrinos, $U$ is a $3 \times 3$ matrix analogous to the CKM matrix for the quarks (Maki, Nakagawa, and Sakata, 1962; Kobayashi and Maskawa, 1973), but due to the Majorana nature of the neutrinos it depends on six independent parameters: three mixing angles and three phases. The two Majorana phases do not affect neutrino oscillations (Bilenky, Hosek, and Petcov, 1980; Langacker, Petcov, Steigman, and Toshev, 1987). [CP conservation implies that the three lepton phases are either zero or $\pi$ (Schechter and Valle, 1981; Wolfenstein, 1981).] This is to be compared to the case of three Dirac neutrinos, in which the number of physical phases is one, similarly to the CKM matrix. Note, however, that the two extra Majorana phases affect only lepton-number-violating processes. Such effects are suppressed by $m_{\nu} / E$ and are very hard to measure.

If no new interactions for the charged leptons are present, we can identify their interaction eigenstates with the corresponding mass eigenstates after phase redefinitions. In this case the charged-current lepton mixing matrix $U$ is simply given by a $3 \times n$ submatrix of the unitary matrix $V^{\nu}$.

\section{NEUTRINO OSCILLATIONS}

\section{A. Neutrino oscillations in vacuum}

Neutrino oscillations in vacuum would arise if neutrinos were massive and mixed. In other words, the neutrino state that is produced by electroweak interactions is not a mass eigenstate. This phenomenon was first pointed out by Pontecorvo in 1957 (Pontecorvo, 1957), while the possibility of arbitrary mixing between two massive neutrino states was first introduced by Maki, Nakagawa, and Sakata (1962).

From Eq. (21) we see that if neutrinos have masses, the weak eigenstates $\nu_{\alpha}$ produced in a weak interaction (i.e., an inverse beta reaction or a weak decay) are, in general, linear combinations of the mass eigenstates $\nu_{i}$,

$$
\left|\nu_{\alpha}\right\rangle=\sum_{i=1}^{n} U_{\alpha i}^{*}\left|\nu_{i}\right\rangle
$$

where $n$ is the number of light neutrino species and $U$ is the mixing matrix in Eq. (24). (Implicit in our definition of the state $|\nu\rangle$ is its energy-momentum and space-time dependence.) After traveling a distance $L$ (or, equivalently for relativistic neutrinos, time $t$ ), a neutrino originally produced with a flavor $\alpha$ evolves as follows:

$$
\left|\nu_{\alpha}(t)\right\rangle=\sum_{i=1}^{n} U_{\alpha i}^{*}\left|\nu_{i}(t)\right\rangle .
$$

It can be detected in the charged-current interaction $\nu_{\alpha}(t) N^{\prime} \rightarrow \ell_{\beta} N$ with a probability
TABLE I. Characteristic values of $L$ and $E$ for various neutrino sources and experiments.

\begin{tabular}{cccc}
\hline \hline Experiment & $L(\mathrm{~m})$ & $E(\mathrm{MeV})$ & $\Delta m^{2}\left(\mathrm{eV}^{2}\right)$ \\
\hline Solar & $10^{10}$ & 1 & $10^{-10}$ \\
Atmospheric & $10^{4}-10^{7}$ & $10^{2}-10^{5}$ & $10^{-1}-10^{-4}$ \\
Reactor & $10^{2}-10^{3}$ & 1 & $10^{-2}-10^{-3}$ \\
Accelerator & $10^{2}$ & $10^{3}-10^{4}$ & $\gtrsim 0.1$ \\
Long-baseline Accelerator & $10^{5}-10^{6}$ & $10^{4}$ & $10^{-2}-10^{-3}$ \\
\hline \hline
\end{tabular}

$$
P_{\alpha \beta}=\left|\left\langle\nu_{\beta} \mid \nu_{\alpha}(t)\right\rangle\right|^{2}=\left|\sum_{i=1}^{n} \sum_{j=1}^{n} U_{\alpha i}^{*} U_{\beta j}\left\langle\nu_{j}(0) \mid \nu_{i}(t)\right\rangle\right|^{2} .
$$

We use the standard approximation that $|\nu\rangle$ is a plane wave (for a pedagogical discussion of the possible quantum-mechanical problems in this naive description of neutrino oscillations we refer the reader to Lipkin, 1999, and Kim and Pevsner, 1993), $\left|\nu_{i}(t)\right\rangle$ $=e^{-i E_{i} t}\left|\nu_{i}(0)\right\rangle$. In all cases of interest to us, the neutrinos are relativistic:

$$
E_{i}=\sqrt{p_{i}^{2}+m_{i}^{2}} \simeq p_{i}+\frac{m_{i}^{2}}{2 E_{i}},
$$

where $E_{i}$ and $m_{i}$ are, respectively, the energy and the mass of the neutrino mass eigenstate $\nu_{i}$. Furthermore, we can assume that $p_{i} \simeq p_{j} \equiv p \simeq E$. Then we obtain the following transition probability (we include here only the $C P$-conserving piece):

$$
P_{\alpha \beta}=\delta_{\alpha \beta}-4 \sum_{i=1}^{n-1} \sum_{j=i+1}^{n} \operatorname{Re}\left[U_{\alpha i} U_{\beta i}^{*} U_{\alpha j}^{*} U_{\beta j}\right] \sin ^{2} x_{i j},
$$

where $x_{i j} \equiv \Delta m_{i j}^{2} L /(4 E)$ with $\Delta m_{i j}^{2} \equiv m_{i}^{2}-m_{j}^{2} . L=t$ is the distance between the source (that is, the production point of $\nu_{\alpha}$ ) and the detector (that is, the detection point of $\nu_{\beta}$ ). In deriving Eq. (29) we used the orthogonality relation $\left\langle\nu_{j}(0) \mid \nu_{i}(0)\right\rangle=\delta_{i j}$. It is convenient to use the following units:

$$
x_{i j}=1.27 \frac{\Delta m_{i j}^{2}}{\mathrm{eV}^{2}} \frac{L / E}{\mathrm{~m} / \mathrm{MeV}} .
$$

The transition probability [Eq. (29)] has an oscillatory behavior, with oscillation lengths

$$
L_{0, i j}^{\mathrm{osc}}=\frac{4 \pi E}{\Delta m_{i j}^{2}}
$$

and amplitude that is proportional to elements in the mixing matrix. Thus, in order to have oscillations, neutrinos must have different masses $\left(\Delta m_{i j}^{2} \neq 0\right)$ and they must mix $\left(U_{\alpha_{i}} U_{\beta i} \neq 0\right)$.

An experiment is characterized by the typical neutrino energy $E$ and by the source-detector distance $L$. In order to be sensitive to a given value of $\Delta m_{i j}^{2}$, the experiment has to be set up with $E / L \approx \Delta m_{i j}^{2}\left(L \sim L_{0, i j}^{\mathrm{osc}}\right)$. The typical values of $L / E$ for different types of neutrino sources and experiments are summarized in Table I. 
If $(E / L) \gg \Delta m_{i j}^{2}\left(L \ll L_{0, i j}^{\mathrm{osc}}\right)$, the oscillation does not have time to give an appreciable effect because $\sin ^{2} x_{i j}$ $\ll 1$. The case of $(E / L) \ll \Delta m_{i j}^{2}\left(L \gg L_{0, i j}^{\text {osc }}\right)$ requires more careful consideration. One must take into account that, in general, neutrino beams are not monochromatic. Thus, rather than measuring $P_{\alpha \beta}$, the experiments are sensitive to the average probability

$$
\begin{aligned}
\left\langle P_{\alpha \beta}\right\rangle & =\frac{\int d E_{\nu} \frac{d \Phi}{d E_{\nu}} \sigma_{C C}\left(E_{\nu}\right) P_{\alpha \beta}\left(E_{\nu}\right) \epsilon\left(E_{\nu}\right)}{\int d E_{\nu} \frac{d \Phi}{d E_{\nu}} \sigma_{C C}\left(E_{\nu}\right) \epsilon\left(E_{\nu}\right)} \\
& =\delta_{\alpha \beta}-4 \sum_{i=1}^{n-1} \sum_{j=i+1}^{n} \operatorname{Re}\left[U_{\alpha i} U_{\beta i}^{*} U_{\alpha j}^{*} U_{\beta j}\right]\left\langle\sin ^{2} x_{i j}\right\rangle,
\end{aligned}
$$

where $\Phi$ is the neutrino energy spectrum, $\sigma_{C C}$ is the cross section for the process in which the neutrino is detected (in general, a charged-current interaction), and $\epsilon\left(E_{\nu}\right)$ is the detection efficiency. For $L \gg L_{0, i j}^{\mathrm{osc}}$, the oscillating phase goes through many cycles before the detection and is averaged to $\left\langle\sin ^{2} x_{i j}\right\rangle=1 / 2$.

For a two-neutrino case, the mixing matrix depends on a single parameter,

$$
U=\left(\begin{array}{cc}
\cos \theta & \sin \theta \\
-\sin \theta & \cos \theta
\end{array}\right),
$$

and there is a single mass-squared difference $\Delta m^{2}$. Then $P_{\alpha \beta}$ of Eq. (29) takes the well-known form

$$
P_{\alpha \beta}=\delta_{\alpha \beta}-\left(2 \delta_{\alpha \beta}-1\right) \sin ^{2} 2 \theta \sin ^{2} x .
$$

The physical parameter space is covered with $\Delta m^{2} \geqslant 0$ and $0 \leqslant \theta \leqslant \pi / 2$ (or, alternatively, $0 \leqslant \theta \leqslant \pi / 4$ and either sign for $\left.\Delta m^{2}\right)$.

Changing the sign of the mass difference, $\Delta m^{2}$ $\rightarrow-\Delta m^{2}$, and changing the octant of the mixing angle, $\theta \rightarrow \pi / 2-\theta$, amounts to redefining the mass eigenstates, $\nu_{1} \leftrightarrow \nu_{2}: P_{\alpha \beta}$ must be invariant under such transformations. Equation (34) reveals, however, that $P_{\alpha \beta}$ is actually invariant under each of these transformations separately. This situation implies that there is a twofold discrete ambiguity in the interpretation of $P_{\alpha \beta}$ in terms of two-neutrino mixing: the two different sets of physical parameters, $\left(\Delta m^{2}, \theta\right)$ and $\left(\Delta m^{2}, \pi / 2-\theta\right)$, give the same transition probability in vacuum. One cannot tell from a measurement of, say, $P_{e \mu}$ in vacuum whether the larger component of $\nu_{e}$ resides in the heavier or in the lighter neutrino mass eigenstate.

Neutrino oscillation experiments measure $P_{\alpha \beta}$. It is common practice for the experiments to interpret their results in the two-neutrino framework. In other words, the constraints on $P_{\alpha \beta}$ are translated into allowed or excluded regions in the plane $\left(\Delta m^{2}, \sin ^{2} 2 \theta\right)$ by using Eq. (34). An example is given in Fig. 1. We now explain some of the typical features of these constraints.

When an experiment is taking data at fixed $\langle L\rangle$ and $\langle E\rangle$, as is the case for most laboratory searches, its result can always be accounted for by $\Delta m^{2}$ that is large enough

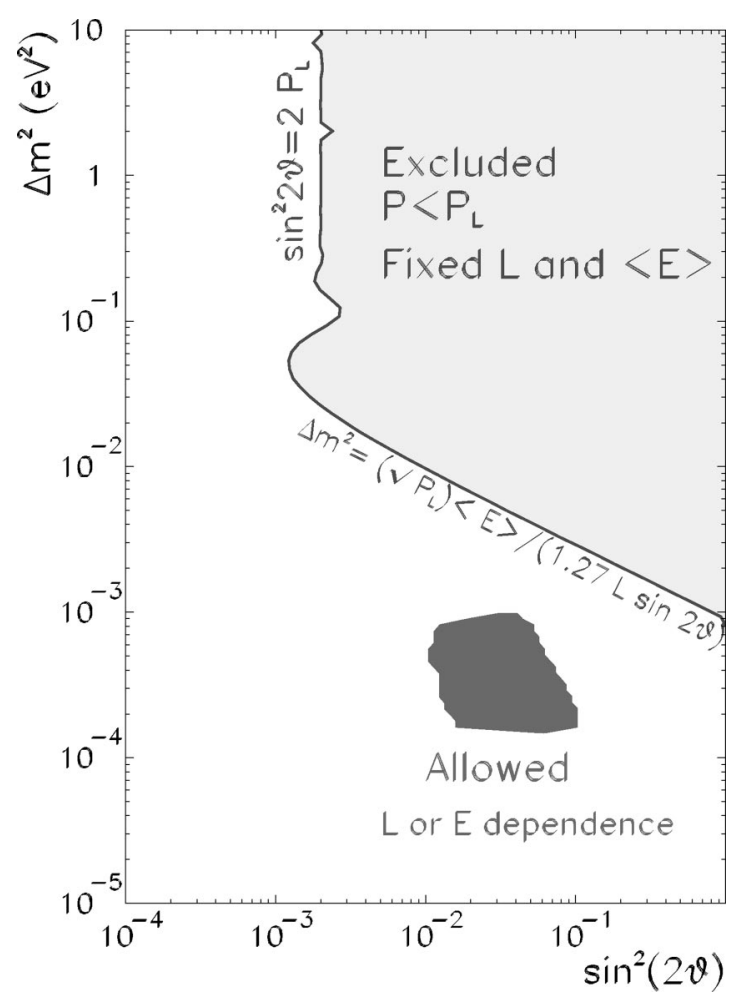

FIG. 1. The characteristic form of an excluded region from a negative search with fixed $L / E$ and of an allowed region from a positive search with varying $L / E$ in the two-neutrino oscillation parameter plane.

to be in the region of averaged oscillations, $\left\langle\sin ^{2} x_{i j}\right\rangle$ $=1 / 2$. Consequently, no upper bound on $\Delta m^{2}$ can be achieved by such an experiment. For negative searches that set an upper bound on the oscillation probability, $\left\langle P_{\alpha \beta}\right\rangle \leqslant P_{L}$, the excluded region always lies on the upper right side of the $\left(\Delta m^{2}, \sin ^{2} 2 \theta\right)$ plane, limited by the following asymptotic lines:

- for $\Delta m^{2} \gg 1 /\langle L / E\rangle$, a vertical line at $\sin ^{2} 2 \theta=2 P_{L}$;

- for $\Delta m^{2} \ll 1 /\langle L / E\rangle$, the oscillating phase can be expanded and the limiting curve takes the form $\Delta m^{2} \sin 2 \theta=4 \sqrt{P_{L}} /\langle L / E\rangle$, which in a log-log plot gives a straight line of slope $-1 / 2$.

If, instead, data are taken at several values of $\langle L\rangle$ and/or $\langle E\rangle$, the corresponding region may be closed, as it is possible to have direct information on the characteristic oscillation wavelength.

\section{B. Neutrinos in matter: Effective potentials}

When neutrinos propagate in dense matter, the interactions with the medium affect their properties. These effects are either coherent or incoherent. For purely incoherent inelastic $\nu-p$ scattering, the characteristic cross section is very small:

$$
\sigma \sim \frac{G_{F}^{2} s}{\pi} \sim 10^{-43} \mathrm{~cm}^{2}\left(\frac{E}{1 \mathrm{MeV}}\right)^{2} .
$$


The smallness of this cross section is demonstrated by the fact that if a beam of $10^{10}$ neutrinos with $E$ $\sim 1 \mathrm{MeV}$ was aimed at the Earth, only one would be deflected by the Earth's matter. It may seem then that for neutrinos matter is irrelevant. However, one must take into account that Eq. (35) does not contain the contribution from forward elastic coherent interactions. In coherent interactions, the medium remains unchanged and it is possible to have interference of scattered and unscattered neutrino waves which enhances the effect. Coherence further allows one to decouple the evolution equation of the neutrinos from the equations of the medium. In this approximation, the effect of the medium is described by an effective potential which depends on the density and composition of the matter (Wolfenstein, 1978).
As an example we derive the effective potential for the evolution of $\nu_{e}$ in a medium with electrons due to its charged-current interactions. The effective low-energy Hamiltonian describing the relevant neutrino interactions is given by

$$
\begin{aligned}
H_{W}= & \frac{G_{F}}{\sqrt{2}}\left[\overline{\nu_{e}}(x) \gamma_{\alpha}\left(1-\gamma_{5}\right) e(x)\right] \\
& \times\left[\bar{e}(x) \gamma_{\alpha}\left(1-\gamma_{5}\right) \nu_{e}(x)\right] .
\end{aligned}
$$

And the effective charged-current Hamiltonian due to the electrons in the medium is

$$
\begin{aligned}
H_{C}^{(e)} & =\frac{G_{F}}{\sqrt{2}} \int d^{3} p_{e} f\left(E_{e}, T\right)\left\langle\left\langle e\left(s, p_{e}\right)\left|\bar{e}(x) \gamma^{\alpha}\left(1-\gamma_{5}\right) \nu_{e}(x) \overline{\nu_{e}}(x) \gamma_{\alpha}\left(1-\gamma_{5}\right) e(x)\right| e\left(s, p_{e}\right)\right\rangle\right\rangle \\
& =\frac{G_{F}}{\sqrt{2}} \overline{\nu_{e}}(x) \gamma_{\alpha}\left(1-\gamma_{5}\right) \nu_{e}(x) \int d^{3} p_{e} f\left(E_{e}, T\right)\left\langle\left\langle e\left(s, p_{e}\right)\left|\bar{e}(x) \gamma_{\alpha}\left(1-\gamma_{5}\right) e(x)\right| e\left(s, p_{e}\right)\right\rangle\right\rangle,
\end{aligned}
$$

where $s$ is the electron spin and $p_{e}$ its momentum. The energy distribution function of the electrons in the medium, $f\left(E_{e}, T\right)$, is assumed to be homogeneous and isotropic and is normalized as $\int d^{3} p_{e} f\left(E_{e}, T\right)=1$. By $\langle\cdots\rangle$ we denote the averaging over electron spinors and summing over all electrons in the medium. Notice that coherence implies that $s, p_{e}$ are the same for initial and final electrons.

Expanding the electron fields $e(x)$ in plane waves we find

$$
\left\langle e\left(s, p_{e}\right)\left|\bar{e}(x) \gamma_{\alpha}\left(1-\gamma_{5}\right) e(x)\right| e\left(s, p_{e}\right)\right\rangle=\frac{1}{V}\left\langle e\left(s, p_{e}\right)\left|\overline{u_{s}}\left(p_{e}\right) a_{s}^{\dagger}\left(p_{e}\right) \gamma_{\alpha}\left(1-\gamma_{5}\right) a_{s}\left(p_{e}\right) u_{s}\left(p_{e}\right)\right| e\left(s, p_{e}\right)\right\rangle,
$$

where $V$ is a volume normalization factor. The averaging gives

$$
\frac{1}{V}\left\langle\left\langle e\left(s, p_{e}\right)\left|a_{s}^{\dagger}\left(p_{e}\right) a_{s}\left(p_{e}\right)\right| e\left(s, p_{e}\right)\right\rangle\right\rangle=N_{e}\left(p_{e}\right) \frac{1}{2} \sum_{s},
$$

where $N_{e}\left(p_{e}\right)$ is the number density of electrons with momentum $p_{e}$. We assumed here that the medium has equal numbers of spin $+1 / 2$ and spin $-1 / 2$ electrons, and we used the fact that $a_{s}^{\dagger}\left(p_{e}\right) a_{s}\left(p_{e}\right)=\mathcal{N}_{e}^{(s)}\left(p_{e}\right)$ is the number operator. We thus obtain

$$
\begin{aligned}
& \left\langle\left\langle e\left(s, p_{e}\right)\left|\bar{e}(x) \gamma_{\alpha}\left(1-\gamma_{5}\right) e(x)\right| e\left(s, p_{e}\right)\right\rangle\right\rangle \\
& \quad=N_{e}\left(p_{e}\right) \frac{1}{2} \sum_{s} \overline{u_{(s)}}\left(p_{e}\right) \gamma_{\alpha}\left(1-\gamma_{5}\right) u_{(s)}\left(p_{e}\right) \\
& \quad=\frac{N_{e}\left(p_{e}\right)}{2} \operatorname{Tr}\left[\frac{m_{e}+p}{2 E_{e}} \gamma_{\alpha}\left(1-\gamma_{5}\right)\right]=N_{e}\left(p_{e}\right) \frac{p_{e}^{\alpha}}{E_{e}} .
\end{aligned}
$$

Isotropy implies that $\int d^{3} p_{e} \vec{p}_{e} f\left(E_{e}, T\right)=0$. Thus only the $p^{0}$ term contributes upon integration, with $\int d^{3} p_{e} f\left(E_{e}, T\right) N_{e}\left(p_{e}\right)=N_{e}$ (the electron number density). Substituting Eq. (40) in Eq. (37) we obtain

$$
H_{C}^{(e)}=\frac{G_{F} N_{e}}{\sqrt{2}} \overline{\nu_{e}}(x) \gamma_{0}\left(1-\gamma_{5}\right) \nu_{e}(x)
$$

The effective potential for $\nu_{e}$ induced by its chargedcurrent interactions with electrons in matter is then given by

$$
\begin{aligned}
V_{C} & =\left\langle\nu_{e}\left|\int d^{3} x H_{C}^{(e)}\right| \nu_{e}\right\rangle=\frac{G_{F} N_{e}}{\sqrt{2}} \frac{2}{V} \int d^{3} x u_{\nu}^{\dagger} u_{\nu} \\
& =\sqrt{2} G_{F} N_{e} .
\end{aligned}
$$

For $\overline{\nu_{e}}$ the sign of $V$ is reversed. This potential can also be expressed in terms of the matter density $\rho$ :

$$
V_{C}=\sqrt{2} G_{F} N_{e} \simeq 7.6 Y_{e} \frac{\rho}{10^{14} \mathrm{~g} / \mathrm{cm}^{3}} \mathrm{eV},
$$

where $Y_{e}=N_{e} /\left(N_{p}+N_{n}\right)$ is the relative number density. Three examples that are relevant to observations are the following:

- At the Earth's core $\rho \sim 10 \mathrm{~g} / \mathrm{cm}^{3}$ and $V_{C} \sim 10^{-13} \mathrm{eV}$;

- At the solar core $\rho \sim 100 \mathrm{~g} / \mathrm{cm}^{3}$ and $V_{C} \sim 10^{-12} \mathrm{eV}$. 
Following the same procedure we can obtain the effective potentials for any flavor neutrino or antineutrino due to interactions with different particles in the medium (for a list, see, for instance, Kim and Pevsner, 1993). For $\nu_{\mu}$ and $\nu_{\tau}, V_{C}=0$ for most media, while for any active neutrino the effective potential due to neutral-current interactions in a neutral medium is $V_{N}$ $=-1 / \sqrt{2} G_{F} N_{n}$, where $N_{n}$ is the number density of neutrons. One can further generalize this analysis to other types of interactions (Bergmann, Grossman, and Nardi, 1999).

\section{Evolution equation in matter: Effective mass and mixing}

There are several derivations in the literature of the evolution equation of a neutrino system in matter (see, for instance, Halprin, 1986; Mannheim, 1988). We follow here the discussion in Baltz and Weneser (1988). Consider a state that is an admixture of two neutrino species $\left|\nu_{e}\right\rangle$ and $\left|\nu_{X}\right\rangle$ or, equivalently, of $\left|\nu_{1}\right\rangle$ and $\left|\nu_{2}\right\rangle$ :

$$
\begin{aligned}
|\Phi(x)\rangle & =\Phi_{e}(x)\left|\nu_{e}\right\rangle+\Phi_{X}(x)\left|\nu_{X}\right\rangle \\
& =\Phi_{1}(x)\left|\nu_{1}\right\rangle+\Phi_{2}(x)\left|\nu_{2}\right\rangle .
\end{aligned}
$$

The evolution of $\Phi$ in a medium is described by a system of coupled Dirac equations:

$$
\begin{aligned}
& E \Phi_{1}=\left[\frac{\hbar}{i} \alpha_{x} \frac{\partial}{\partial x}+\beta m_{1}+V_{11}\right] \Phi_{1}+V_{12} \Phi_{2}, \\
& E \Phi_{2}=\left[\frac{\hbar}{i} \alpha_{x} \frac{\partial}{\partial x}+\beta m_{2}+V_{22}\right] \Phi_{2}+V_{12} \Phi_{1},
\end{aligned}
$$

where $\beta=\gamma_{0}$ and $\alpha_{x}=\gamma_{0} \gamma_{1}$. The $V_{i j}$ terms give the effective potential for neutrino mass eigenstates. They can be simply derived from the effective potential for interaction eigenstates [such as $V_{e e}$ of Eq. (42)]:

$$
V_{i j}=\left\langle\nu_{i}\left|\int d^{3} x H_{i n t}^{\text {medium }}\right| \nu_{j}\right\rangle=U_{i \alpha} V_{\alpha \alpha} U_{j \alpha}^{*} .
$$

We decompose the neutrino state: $\Phi_{i}(x)=C_{i}(x) \phi_{i}(x)$. Here $\phi_{i}(x)$ is the Dirac spinor part satisfying

$$
\left(\alpha_{x}\left\{\left[E-V_{i i}(x)\right]^{2}-m_{i}^{2}\right\}^{1 / 2}+\beta m_{i}+V_{i i}\right) \phi_{i}(x)=E \phi_{i}(x) \text {. }
$$

So $\phi_{i}(x)$ has the form of a free-particle solution with local energy $\mathcal{E}_{i}(x)=E-V_{i i}(x)$ :

$$
\phi_{i}(x)=\left[\frac{\mathcal{E}_{i}+m_{i}}{2 \mathcal{E}_{i}}\right]^{1 / 2} \times\left[\begin{array}{c}
\chi \\
\frac{\sqrt{\mathcal{E}_{i}^{2}-m_{i}^{2}}}{\mathcal{E}_{i}+m_{i}} \sigma_{x} \chi
\end{array}\right],
$$

where $\chi$ is the Pauli spinor. We make the following approximations:

(i) The scale over which $V$ changes is much larger than the microscopic wavelength of the neutrino: $(\partial V / \partial x) / V \ll \hbar m / E^{2}$.

(ii) Expanding to first order in $V$ implies that $V_{12} \alpha_{x} \phi_{2}$ $\simeq \phi_{1}, \quad V_{12} \alpha_{x} \phi_{1} \simeq \phi_{2}$, and $\left\{\left[E-V_{i i}(x)\right]^{2}-m_{i}^{2}\right\}^{1 / 2}$ $\simeq E-V_{i i}(x)-m_{i}^{2} / 2 E$.

From (i) we find that the Dirac equations take the form

$E C_{1} \phi_{1}=\frac{\hbar}{i} \alpha_{x} \frac{\partial C_{1}}{\partial x} \phi_{1}+\left(\beta m_{1}+V_{11}\right) C_{1} \phi_{1}+V_{12} C_{2} \phi_{2}$,

$E C_{2} \phi_{2}=\frac{\hbar}{i} \alpha_{x} \frac{\partial C_{2}}{\partial x} \phi_{2}+\left(\beta m_{2}+V_{22}\right) C_{2} \phi_{2}+V_{12} C_{1} \phi_{1}$.

Then multiplying by $\alpha_{x}$ and using the equation of motion of $\phi_{i}$ and (ii), we can drop the dependence on the spinor $\phi$ and obtain

$$
\begin{aligned}
& \frac{\hbar}{i} \frac{\partial C_{1}}{\partial x}=\left(E-V_{11}(x)-\frac{m_{1}^{2}}{2 E}\right) C_{1}-V_{12} C_{2}, \\
& \frac{\hbar}{i} \frac{\partial C_{2}}{\partial x}=\left(E-V_{22}(x)-\frac{m_{2}^{2}}{2 E}\right) C_{2}-V_{12} C_{1} .
\end{aligned}
$$

Changing notations $C_{i, \alpha}(x) \rightarrow \nu_{i, \alpha}(x)$ (and $\hbar=1$ ), removing the diagonal piece that is proportional to $E$, and rotating to the flavor basis, we can rewrite Eq. (50) in matrix form (Wolfenstein, 1978):

$$
-i \frac{\partial}{\partial x}\left(\begin{array}{c}
\nu_{e} \\
\nu_{X}
\end{array}\right)=\left(-\frac{M_{w}^{2}}{2 E}\right)\left(\begin{array}{c}
\nu_{e} \\
\nu_{X}
\end{array}\right)
$$

where we have defined an effective mass matrix in matter

$$
M_{w}^{2}=\left(\begin{array}{cc}
\frac{m_{1}^{2}+m_{2}^{2}}{2}+2 E V_{e}-\frac{\Delta m^{2}}{2} \cos 2 \theta & \frac{\Delta m^{2}}{2} \sin 2 \theta \\
\frac{\Delta m^{2}}{2} \sin 2 \theta & \frac{m_{1}^{2}+m_{2}^{2}}{2}+2 E V_{X}+\frac{\Delta m^{2}}{2} \cos 2 \theta
\end{array}\right)
$$

Here $\Delta m^{2}=m_{2}^{2}-m_{1}^{2}$.

We define the instantaneous mass eigenstates in matter, $\nu_{i}^{m}$, as the eigenstates of $M_{w}$ for a fixed value of $x$ (or $t$ ). They are related to the interaction eigenstates through a unitary rotation,

$$
\left(\begin{array}{c}
\nu_{e} \\
\nu_{X}
\end{array}\right)=U\left(\theta_{m}\right)\left(\begin{array}{c}
\nu_{1}^{m} \\
\nu_{2}^{m}
\end{array}\right)=\left(\begin{array}{cc}
\cos \theta_{m} & \sin \theta_{m} \\
-\sin \theta_{m} & \cos \theta_{m}
\end{array}\right)\left(\begin{array}{c}
\nu_{1}^{m} \\
\nu_{2}^{m}
\end{array}\right)
$$

The eigenvalues of $M_{w}$, that is, the effective masses in 


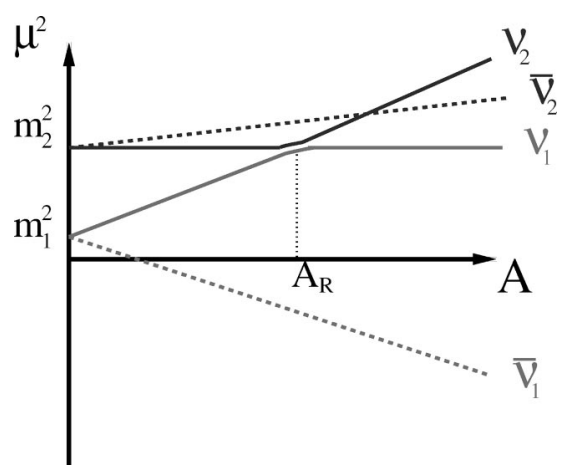

FIG. 2. Effective masses acquired in the medium by a system of two massive neutrinos as a function of the potential $A$ [see Eq. (54)].

matter are given by (Wolfenstein, 1978; Mikheyev and Smirnov, 1985)

$$
\begin{aligned}
\mu_{1,2}^{2}(x)= & \frac{m_{1}^{2}+m_{2}^{2}}{2}+E\left(V_{e}+V_{X}\right) \\
& \mp \frac{1}{2} \sqrt{\left(\Delta m^{2} \cos 2 \theta-A\right)^{2}+\left(\Delta m^{2} \sin 2 \theta\right)^{2}},
\end{aligned}
$$

while the mixing angle in matter is given by

$$
\tan 2 \theta_{m}=\frac{\Delta m^{2} \sin 2 \theta}{\Delta m^{2} \cos 2 \theta-A} \text {. }
$$

The quantity $A$ is defined by

$$
A \equiv 2 E\left(V_{e}-V_{X}\right) \text {. }
$$

In Figs. 2 and 3 we plot, respectively, the effective masses and the mixing angle in matter as functions of the potential $A$, for $A>0$ and $\Delta m^{2} \cos 2 \theta>0$. Notice that even massless neutrinos acquire nonvanishing effective masses in matter.

The resonant density (or potential) $A_{R}$ is defined as the value of $A$ for which the difference between the effective masses is minimal:

$$
A_{R}=\Delta m^{2} \cos 2 \theta \text {. }
$$

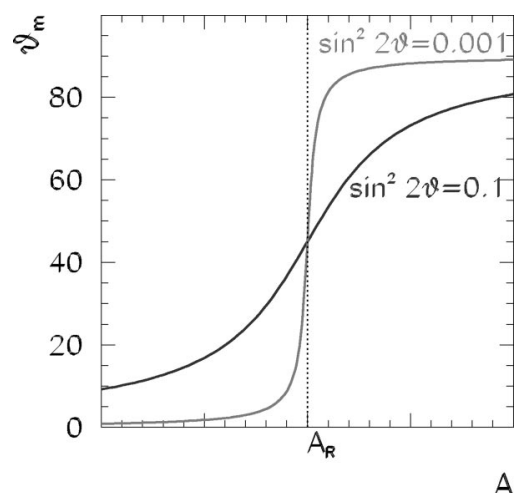

FIG. 3. The mixing angle in matter for a system of two massive neutrinos as a function of the potential $A$ for two different values of the mixing angle in vacuum [see Eq. (55)].
Notice that once the sign of $V_{e}-V_{X}$ (which depends on the composition of the medium and on the state $X$ ) is known, this resonance condition can only be achieved for a given sign of $\Delta m^{2} \cos 2 \theta$, i.e., for mixing angles in only one of the two possible octants. We learn that the symmetry present in vacuum oscillations is broken by matter potentials. Also, if the resonant condition is achieved for two neutrinos, it cannot be achieved for antineutrinos of the same flavor, and vice versa. The mixing angle $\tan 2 \theta_{m}$ changes sign at $A_{R}$. As can be seen in Fig. 3, for $A>A_{R}$ we have $\theta_{m} \gg \theta$.

We define an oscillation length in matter

$$
L^{\mathrm{osc}}=\frac{L_{0}^{\mathrm{osc}} \Delta m^{2}}{\sqrt{\left(\Delta m^{2} \cos 2 \theta-A\right)^{2}+\left(\Delta m^{2} \sin 2 \theta\right)^{2}}},
$$

where the oscillation length in vacuum, $L_{0}^{\text {osc }}$, was defined in Eq. (31). The oscillation length in matter presents a resonant behavior. At the resonance point the oscillation length is

$$
L_{R}^{\mathrm{osc}}=\frac{L_{0}^{\mathrm{osc}}}{\sin 2 \theta} .
$$

The width (in distance) of the resonance, $\delta r_{R}$, corresponding to $\delta A_{R}=2 \Delta m^{2} \sin ^{2} 2 \theta$, is given by

$$
\delta r_{R}=\frac{\delta A_{R}}{\left|\frac{d A}{d r}\right|_{R}}=\frac{2 \tan 2 \theta}{h_{R}}, \quad h_{R} \equiv\left|\frac{1}{A} \frac{d A}{d r}\right|_{R},
$$

where we have defined the resonance height $h_{R}$.

For constant $A$, i.e., for constant matter density, the evolution of the neutrino system is described just in terms of the masses and mixing in matter. But for varying $A$, this is in general not the case.

\section{Adiabatic versus nonadiabatic transitions}

Taking time derivative of Eq. (53), we find

$$
\frac{\partial}{\partial t}\left(\begin{array}{c}
\nu_{e} \\
\nu_{X}
\end{array}\right)=\dot{U}\left(\theta_{m}\right)\left(\begin{array}{c}
\nu_{1}^{m} \\
\nu_{2}^{m}
\end{array}\right)+U\left(\theta_{m}\right)\left(\begin{array}{c}
\dot{\nu}_{1}^{m} \\
\dot{\nu}_{2}^{m}
\end{array}\right) .
$$

Using the evolution equation in the flavor basis, Eq. (51), we get

$$
\begin{aligned}
i\left(\begin{array}{c}
\dot{\nu}_{1}^{m} \\
\dot{\nu}_{2}^{m}
\end{array}\right)= & \frac{1}{2 E} U^{\dagger}\left(\theta_{m}\right) M_{w}^{2} U\left(\theta_{m}\right)\left(\begin{array}{c}
\nu_{1}^{m} \\
\nu_{2}^{m}
\end{array}\right) \\
& -i U^{\dagger} \dot{U}\left(\theta_{m}\right)\left(\begin{array}{c}
\nu_{1}^{m} \\
\nu_{2}^{m}
\end{array}\right) .
\end{aligned}
$$

For constant matter density, $\theta_{m}$ is constant and the second term vanishes. In general, using the definition of the effective masses $\mu_{i}(t)$ in Eq. (54) and subtracting a diagonal piece $\left(\mu_{1}^{2}+\mu_{2}^{2}\right) / 2 E \times I$, we can rewrite the evolution equation as

$$
i\left(\begin{array}{c}
\dot{\nu}_{1}^{m} \\
\dot{\nu}_{2}^{m}
\end{array}\right)=\frac{1}{4 E}\left(\begin{array}{cc}
-\Delta(t) & -4 i E \dot{\theta}_{m}(t) \\
4 i E \dot{\theta}_{m}(t) & \Delta(t)
\end{array}\right)\left(\begin{array}{c}
\nu_{1}^{m} \\
\nu_{2}^{m}
\end{array}\right),
$$

where we defined $\Delta(t) \equiv \mu_{2}^{2}(t)-\mu_{1}^{2}(t)$. 
The evolution equations (63) constitute a system of coupled equations: the instantaneous mass eigenstates $\nu_{i}^{m}$ mix in the evolution and are not energy eigenstates. The importance of this effect is controlled by the relative size of the off-diagonal piece $4 E \dot{\theta}_{m}(t)$ with respect to the diagonal one $\Delta(t)$. When $\Delta(t) \gg 4 E \dot{\theta}_{m}(t)$, the instantaneous mass eigenstates, $\nu_{i}^{m}$, behave approximately as energy eigenstates and they do not mix in the evolution. This is the adiabatic transition approximation. From the definition of $\theta_{m}$ in Eq. (55), we find

$$
\dot{\theta}_{m}=\frac{\Delta m^{2} \sin 2 \theta}{2 \Delta(t)^{2}} \dot{A}
$$

The adiabaticity condition then reads

$$
\Delta(t) \gg \frac{2 E A \Delta m^{2} \sin 2 \theta}{\Delta(t)^{2}}\left|\frac{\dot{A}}{A}\right| \text {. }
$$

Since for small mixing angles the maximum of $\dot{\theta}_{m}$ occurs at the resonance point (as can be seen in Fig. 3), the strongest adiabaticity condition is obtained when Eq. (65) is evaluated at the resonance (the generalization of the condition of maximum adiabaticity violation to large mixings can be found in Friedland, 2001 and Lisi et al. 2001). We define the adiabaticity parameter $Q$ at the resonance as follows:

$$
Q=\frac{\Delta m^{2} \sin ^{2} 2 \theta}{E \cos 2 \theta h_{R}}=\frac{4 \pi \delta r_{R}}{L_{R}^{o s c}},
$$

where we used the definitions of $A_{R}, \delta r_{R}$, and $h_{R}$ in Eqs. (57) and (60). Writing it in this form, we see that the adiabaticity condition, $Q \gg 1$, implies that many oscillations take place in the resonant region. Conversely, when $Q \lesssim 1$ the transition is nonadiabatic.

The survival amplitude of a $\nu_{e}$ produced in matter at $t_{0}$ and exiting the matter at $t>t_{0}$ can be written as follows:

$$
\begin{aligned}
A\left(\nu_{e} \rightarrow \nu_{e} ; t\right)= & \sum_{i, j} A\left[\nu_{e}\left(t_{0}\right) \rightarrow \nu_{i}\left(t_{0}\right)\right] \\
& \times A\left[\nu_{i}\left(t_{0}\right) \rightarrow \nu_{j}(t)\right] A\left[\nu_{j}(t) \rightarrow \nu_{e}(t)\right]
\end{aligned}
$$

with

$$
\begin{aligned}
& A\left[\nu_{e}\left(t_{0}\right) \rightarrow \nu_{i}\left(t_{0}\right)\right]=\left\langle\nu_{i}\left(t_{0}\right) \mid \nu_{e}\left(t_{0}\right)\right\rangle=U_{e i}^{*}\left(\theta_{m, 0}\right), \\
& A\left[\nu_{j}(t) \rightarrow \nu_{e}(t)\right]=\left\langle\nu_{e}(t) \mid \nu_{j}(t)\right\rangle=U_{e j}(\theta),
\end{aligned}
$$

where $U_{e i}^{*}\left(\theta_{m, 0}\right)$ is the $(e i)$ element of the mixing matrix in matter at the production point and $U_{e j}(\theta)$ is the $(e j)$ element of the mixing matrix in vacuum.

In the adiabatic approximation the mass eigenstates do not mix, so

$$
\begin{aligned}
A\left[\nu_{i}\left(t_{0}\right) \rightarrow \nu_{j}(t)\right] & =\delta_{i j}\left\langle\nu_{i}(t) \mid \nu_{i}\left(t_{0}\right)\right\rangle \\
& =\delta_{i j} \exp \left\{i \int_{t_{0}}^{t} E_{i}\left(t^{\prime}\right) d t^{\prime}\right\} .
\end{aligned}
$$

Note that $E_{i}$ is a function of time because the effective mass $\mu_{i}$ is a function of time,

$$
E_{i}\left(t^{\prime}\right) \simeq p+\frac{\mu_{i}^{2}\left(t^{\prime}\right)}{2 p} .
$$

Thus the transition probability for the adiabatic case is given by

$$
\begin{aligned}
P\left(\nu_{e}\right. & \left.\rightarrow \nu_{e} ; t\right) \\
& =\left|\sum_{i} U_{e i}(\theta) U_{e i}^{*}\left(\theta_{m, 0}\right) \exp \left(-\frac{i}{2 E} \int_{t_{0}}^{t} \mu_{i}^{2}\left(t^{\prime}\right) d t^{\prime}\right)\right|^{2} .
\end{aligned}
$$

For the case of two-neutrino mixing, Eq. (70) takes the form

$$
\begin{aligned}
P\left(\nu_{e} \rightarrow \nu_{e} ; t\right)= & \cos ^{2} \theta_{m} \cos ^{2} \theta+\sin ^{2} \theta_{m} \sin ^{2} \theta \\
& +\frac{1}{2} \sin 2 \theta_{m} \sin 2 \theta \cos \left(\frac{\delta(t)}{2 E}\right),
\end{aligned}
$$

where

$$
\begin{aligned}
\delta(t) & =\int_{t_{0}}^{t} \Delta\left(t^{\prime}\right) d t^{\prime} \\
& =\int_{t_{0}}^{t} \sqrt{\left[\Delta m^{2} \cos 2 \theta-A\left(t^{\prime}\right)\right]^{2}+\left(\Delta m^{2} \sin 2 \theta\right)^{2}} d t^{\prime},
\end{aligned}
$$

which, in general, has to be evaluated numerically. There are some analytical approximations for specific forms of $A\left(t^{\prime}\right)$ : exponential, linear, etc. (see, for instance, Kuo and Pantaleone, 1989). For $\delta(t) \gg E$ the last term in Eq. (71) is averaged out and the survival probability takes the form

$$
P\left(\nu_{e} \rightarrow \nu_{e} ; t\right)=\frac{1}{2}\left[1+\cos 2 \theta_{m} \cos 2 \theta\right] .
$$

In Fig. 4 we plot isocontours of constant survival probability in the parameter plane $\left(\Delta m^{2}, \tan ^{2} \theta\right)$ for the particular case of the Sun's density, for which $A>0$. Notice that, unlike $\sin ^{2} 2 \theta, \tan ^{2} \theta$ is a single-valued function in the full parameter range $0 \leqslant \theta \leqslant \pi / 2$. Therefore it is a more appropriate variable once matter effects are included and the symmetry of the survival probability with respect to the change of octant for the mixing angle is lost. As can be seen in the figure, for $\theta<\pi / 4, P\left(\nu_{e}\right.$ $\rightarrow \nu_{e}$ ) in matter can be larger or smaller than $1 / 2$, in contrast to the case of vacuum oscillations where, in the averaged regime, $P_{e e}^{v a c}=1-\frac{1}{2} \sin ^{2} 2 \theta>\frac{1}{2}$.

In Fig. 4 we also plot the limiting curve for $Q=1$. To the left of and below this curve, the adiabatic approximation breaks down and the isocontours in Fig. 4 deviate from the expression in Eq. (73). In this region, the off-diagonal term $\dot{\theta}_{m}$ cannot be neglected, and the mixing between instantaneous mass eigenstates is important. In this case we can write

$$
\begin{aligned}
A\left[\nu_{i}\left(t_{0}\right) \rightarrow \nu_{j}(t)\right]= & \left\langle\nu_{j}(t) \mid \nu_{j}\left(t_{R}\right)\right\rangle\left\langle\nu_{j}\left(t_{R}\right) \mid \nu_{i}\left(t_{R}\right)\right\rangle \\
& \times\left\langle\nu_{i}\left(t_{R}\right) \mid \nu_{i}\left(t_{0}\right)\right\rangle,
\end{aligned}
$$

where $t_{R}$ is the point of maximum adiabaticity violation, which, for small mixing angles, corresponds to the reso- 


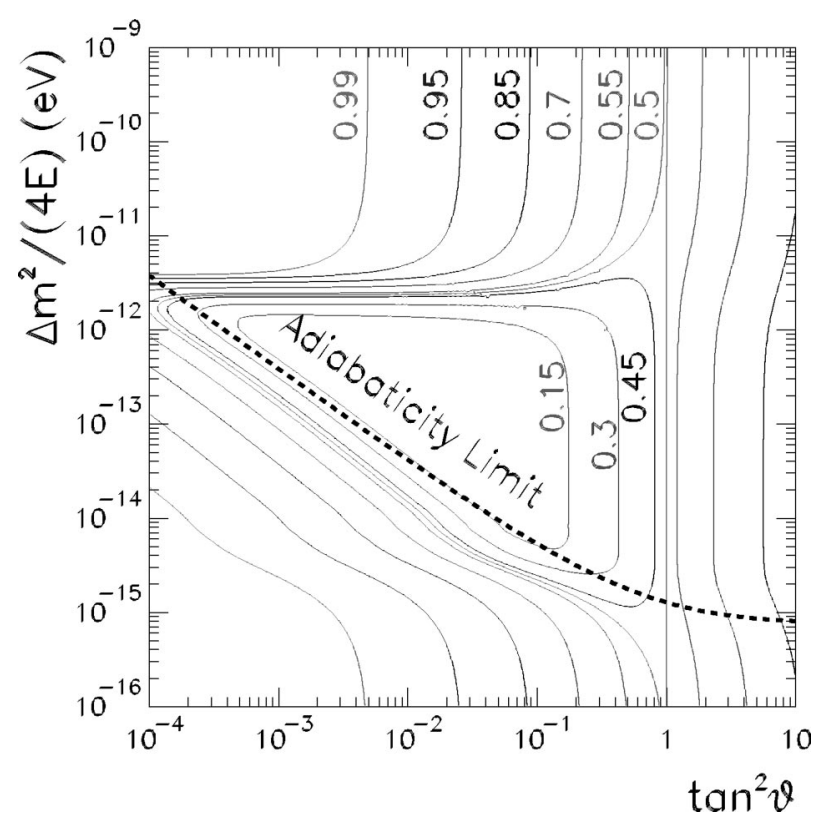

FIG. 4. Isocontours of the survival probability $P_{e e}$ in the Sun. Also shown is the limit of applicability of the adiabatic approximation $Q=1$ (dashed line).

nant point. The possibility of this level crossing can be described in terms of the Landau-Zener probability (Landau, 1932; Zener, 1932):

$$
P_{L Z}=\left|\left\langle\nu_{j}\left(t_{R}\right) \mid \nu_{i}\left(t_{R}\right)\right\rangle\right|^{2} \quad(i \neq j) .
$$

Introducing this transition probability in Eq. (67) we find that in the nonadiabatic regime (after averaging out the oscillatory term), the survival probability can be written as

$$
P\left(\nu_{e} \rightarrow \nu_{e} ; t\right)=\frac{1}{2}\left[1+\left(1-2 P_{L Z}\right) \cos 2 \theta_{m} \cos 2 \theta\right] .
$$

The physical interpretation of this expression is straightforward. An electron neutrino produced at $A>A_{R}$ consists of an admixture of $\nu_{1}$ with fraction $\cos ^{2} \theta_{m}$ and $\nu_{2}$ with fraction $\sin ^{2} \theta_{m}$. In particular, for very small mixing angles in vacuum, $\theta_{m} \sim \pi / 2$ (see Fig. 3) so $\nu_{e}$ is almost a pure $\nu_{2}\left(t_{0}\right)$ state. When the neutrino state reaches resonance, $\nu_{2}\left(\nu_{1}\right)$ can become $\nu_{2}\left(\nu_{1}\right)$ with probability [1 $\left.-P_{L Z}\right]$ or $\nu_{1}\left(\nu_{2}\right)$ with probability $P_{L Z}$. So after passing the resonance, the $\nu_{e}$ flux contains a fraction of $\nu_{1}$ : $P_{e 1}=\sin ^{2} \theta_{m} P_{L Z}+\cos ^{2} \theta_{m}\left(1-P_{L Z}\right)$, and a fraction of $\nu_{2}$ : $P_{e 2}=\cos ^{2} \theta_{m} P_{L Z}+\sin ^{2} \theta_{m}\left(1-P_{L Z}\right)$. At the exit, $\nu_{1}$ consists of $\nu_{e}$ with fraction $\cos ^{2} \theta$, and $\nu_{2}$ consists of $\nu_{e}$ with fraction $\sin ^{2} \theta$ so (Haxton, 1986; Parke, 1986; Petcov, 1987) $P_{e e}=\cos ^{2} \theta P_{e 1}+\sin ^{2} \theta P_{e 2}$ which reproduces Eq. (76).

The Landau-Zener probability can be evaluated in the WKB approximation (for details see, for instance, Kim and Pevsner, 1993). The general form of the LandauZener probability for an exponential density can be written as (Krastev and Petcov, 1988; Petcov, 1988a)

$$
P_{L Z}=\frac{\exp \left(-\gamma \sin ^{2} \theta\right)-\exp (-\gamma)}{1-\exp (-\gamma)},
$$

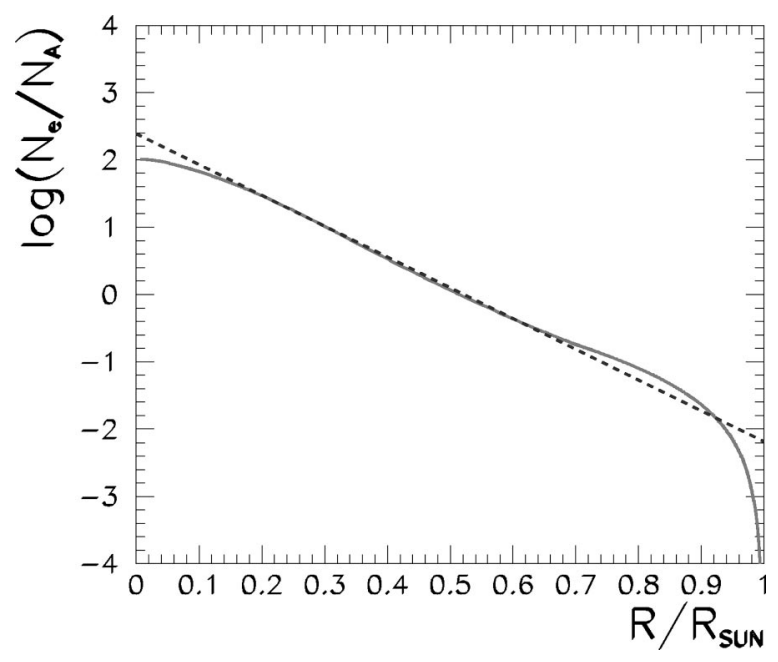

FIG. 5. The solar matter density profile: solid line, for the model of Bahcall, Pinsonneault, and Basu (2001); dashed line, the exponential approximation.

$$
\gamma \equiv \pi \frac{\Delta m^{2}}{E|\dot{A} / A|_{R}}=\pi Q \frac{\cos 2 \theta}{\sin ^{2} 2 \theta} .
$$

When $\nu_{e}$ is produced at $A \gg A_{R}$ and $\theta$ is small, $\theta_{m} \sim 90^{\circ}$. In this case $\gamma$ is very large and $P_{L Z}$ $\simeq \exp \left(-\gamma \sin ^{2} \theta\right) \simeq \exp [-(\pi / 4) Q], \quad$ and the survival probability is simply given by

$$
P\left(\nu_{e} \rightarrow \nu_{e} ; t\right) \simeq P_{L Z} \simeq \exp \left(-\frac{\pi}{4} Q\right),
$$

where $Q$ is the adiabaticity parameter defined in Eq. (66). Since $Q \sim \Delta m^{2} \sin ^{2} 2 \theta / E$, the isocontours of constant probability in this regime correspond to diagonal lines in the $\left(\Delta m^{2}, \tan ^{2} \theta\right)$ plane in a log-log plot, as illustrated in Fig. 4.

\section{E. Propagation in the sun: Mikheyev-Smirnov-Wolfenstein effect}

As an illustration of the matter effects discussed in the previous section we describe now the propagation of a $\nu_{e}-\nu_{X}$ neutrino system in the matter density of the Sun. For the sake of concreteness we assume that $X$ is some superposition of $\mu$ and $\tau$.

The solar density distribution decreases monotonically (see Fig. 5). For $R<0.9 R_{\odot}$ it can be approximated by an exponential $N_{e}(R)=N_{e}(0) \exp \left(-R / r_{0}\right)$, with $r_{0}$ $=R_{\odot} / 10.54=6.6 \times 10^{7} \mathrm{~m}=3.3 \times 10^{14} \mathrm{eV}^{-1}$.

After traversing this density, the dominant component of the exiting neutrino state depends on the value of the mixing angle in vacuum and the relative size of $\Delta m^{2} \cos 2 \theta$ versus $A_{0}=2 E G_{F} N_{e, 0}$ (at the neutrino production point):

(1) $\Delta m^{2} \cos 2 \theta \gg A_{0}$ : matter effects are negligible and the propagation occurs as in vacuum. The survival probability at the sunny surface of the Earth is

$$
P_{e e}\left(\Delta m^{2} \cos 2 \theta \gg A_{0}\right)=1-\frac{1}{2} \sin ^{2} 2 \theta>\frac{1}{2} .
$$


(2) $\Delta m^{2} \cos 2 \theta \geq A_{0}$ : the neutrino does not pass through the resonance point but its mixing is affected by the matter in the Sun. This effect is well described by an adiabatic propagation:

$$
P_{e e}\left(\Delta m^{2} \cos 2 \theta \gtrsim A_{0}\right)=\frac{1}{2}\left(1+\cos 2 \theta_{m} \cos 2 \theta\right) .
$$

Since the resonance point is not crossed, $\cos 2 \theta_{m}$ has the same sign as $\cos 2 \theta$ and the corresponding survival probability is also larger than $1 / 2$.

(3) $\Delta m^{2} \cos 2 \theta<A_{0}$ : the neutrino can cross the resonance point on its way out. In this case, as discussed in the previous section, for small mixing angle in vacuum, $\nu_{e} \sim \nu_{2}^{m}$ at the production point and remains $\nu_{2}^{m}$ till the resonance point (for larger mixing but still in the first octant, $\nu_{e}$ is a combination of $\nu_{1}^{m}$ and $\nu_{2}^{m}$ with larger $\nu_{2}^{m}$ component). It is important in this case to find whether the transition is adiabatic. For the solar density, $Q \sim 1$ corresponds to

$$
\frac{\left(\Delta m^{2} / \mathrm{eV}^{2}\right) \sin ^{2} 2 \theta}{(E / \mathrm{MeV}) \cos 2 \theta} \sim 3 \times 10^{-9} .
$$

For $Q \gg 1$ the transition is adiabatic and the neutrino state remains in the same linear combination of mass eigenstates after the resonance determined by $\theta_{m}$. As can be seen in Fig. 3, $\theta_{m}$ (that is, the $\nu_{e}$ component of the state) decreases after crossing the resonance and, consequently, so does the survival probability $P_{e e}$. In particular, for small mixing angle, $\nu_{2}$ at the exit point is almost a pure $\nu_{X}$ and, consequently, $P_{e e}$ can be very small. Explicitly,

$P_{e e}\left(\Delta m^{2} \cos 2 \theta<A_{0}, Q \gg 1\right)=\frac{1}{2}\left(1+\cos 2 \theta_{m, 0} \cos 2 \theta\right)$

can be much smaller than $1 / 2$ because $\cos 2 \theta_{m, 0}$ and $\cos 2 \theta \operatorname{can}$ have opposite signs. Note that the smaller the mixing angle in vacuum the larger is the deficit of electron neutrinos in the outgoing state. This is the Mikheyev-Smirnov-Wolfenstein (MSW) effect (Wolfenstein, 1978; Mikheyev and Smirnov, 1985). This behavior is illustrated in Fig. 6, where we plot the electron survival probability as a function of $\Delta m^{2} / E$ for different values of the mixing angle.

For smaller values of $\Delta m^{2} / E$ (right side of Fig. 6) we approach the regime where $Q<1$ and nonadiabatic effects start playing a role. In this case the state can jump from $\nu_{2}$ into $\nu_{1}$ (or vice versa) with probability $P_{L Z}$. For small mixing angle, at the surface $\nu_{1} \sim \nu_{e}$ and the $\nu_{e}$ component of the exiting neutrino increases. This can be seen from the expression for $P_{e e}$,

$$
\begin{aligned}
P_{e e} & \left(\Delta m^{2} \cos 2 \theta<A_{0}, Q \ll 1\right) \\
= & \frac{1}{2}\left[1+\left(1-2 P_{L Z}\right) \cos 2 \theta_{m} \cos 2 \theta\right],
\end{aligned}
$$

and from Fig. 6. For large mixing angles this expression is still valid.

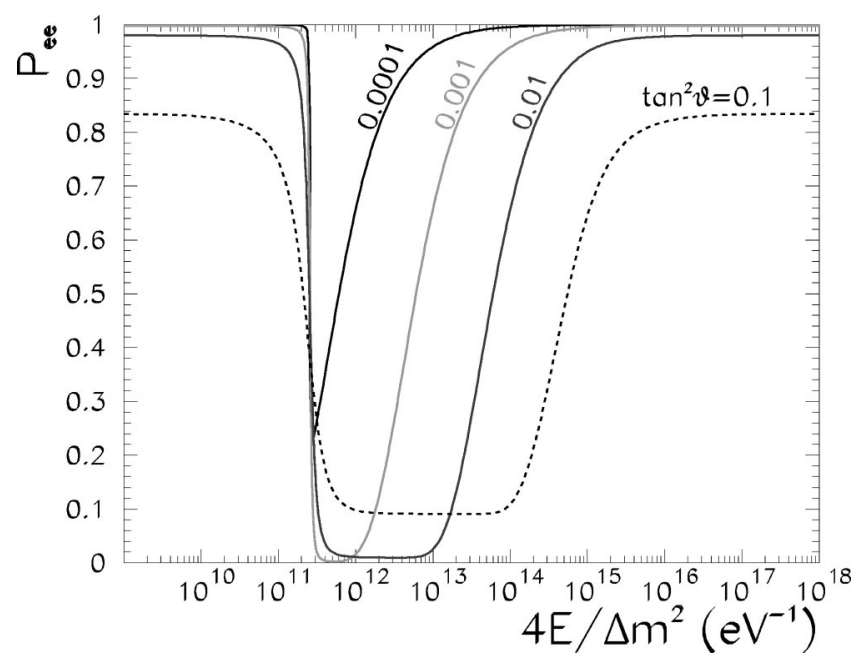

FIG. 6. The survival probability for a $\nu_{e}$ state produced in the center of the Sun as a function of $E / \Delta m^{2}$ for various values of the mixing angle.

\section{SOLAR NEUTRINOS}

Solar neutrinos are electron neutrinos produced in the thermonuclear reactions which generate the solar energy. These reactions occur via two main chains, the $p p$ chain and the CNO cycle, shown in Figs. 7 and 8, respectively. There are five reactions which produce $\nu_{e}$ in the $p p$ chain and three in the $\mathrm{CNO}$ cycle. Both chains result in the overall fusion of protons into ${ }^{4} \mathrm{He}$ :

$$
4 p \rightarrow{ }^{4} \mathrm{He}+2 e^{+}+2 \nu_{e}+\gamma,
$$

where the energy released in the reaction, $Q=4 m_{p}$ $-m_{4} \mathrm{He}^{-2} m_{e} \simeq 26 \mathrm{MeV}$, is mostly radiated through the photons and only a small fraction is carried by the neutrinos, $\left\langle E_{2 v_{e}}\right\rangle=0.59 \mathrm{MeV}$.

In order to determine precisely the rates of the different reactions in the two chains, which would give us the final neutrino fluxes and their energy spectrum, a detailed knowledge of the Sun and its evolution is needed. Solar models (Bahcall and Ulrich, 1988; Turck-Chieze, Cahen, Casse, and Doom, 1988; Bahcall and Pinsonneault, 1992, 1995; Bahcall, Basu, and Pinsonneault, 1998; Bahcall, Pinsonneault, and Basu, 2001) describe

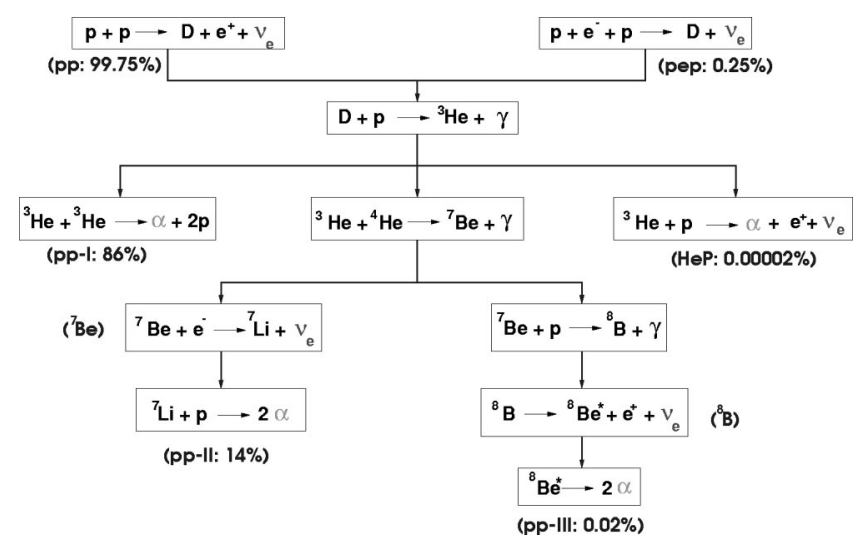

FIG. 7. The $p p$ chain in the Sun. 


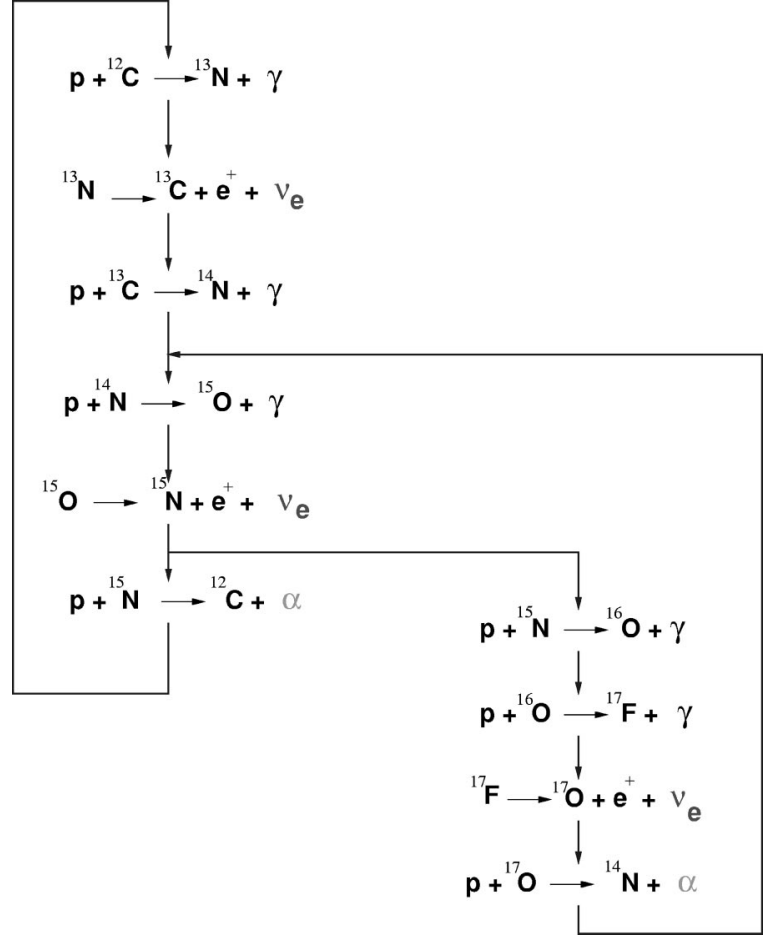

FIG. 8. The CNO cycle in the Sun.

the properties of the Sun and its evolution after entering the main sequence. The models are based on a set of observational parameters: surface luminosity (3.844 $\left.\times 10^{26} \mathrm{~W}\right)$, age $\left(4.5 \times 10^{9} \mathrm{yr}\right)$, radius $\left(6.961 \times 10^{8} \mathrm{~m}\right)$ and mass $\left(1.989 \times 10^{30} \mathrm{~kg}\right)$, and on several basic assumptions: spherical symmetry, hydrostatic and thermal equilibrium, equation of state of an ideal gas, and present surface abundances of elements similar to the primordial composition. Over the past four decades, the solar models have been steadily refined as the result of increased observational and experimental information about the input parameters (such as nuclear reaction rates and the surface abundances of different elements), more accurate calculations of constituent quantities (such as radiative opacity and equation of state), inclusion of new physical effects (such as element diffusion), and development of faster computers and more precise stellar evolution codes.

We use as Standard Solar Model (SSM), the most updated version of the model developed by Bahcall, Pinsonneault, and Basu, (2001, sometimes referred to as BP00). (At present, the various solar models give essentially the same results when interpreting the bulk of experimental data in terms of neutrino parameters. In this sense, the implications for particle physics discussed in this review are independent of the choice of solar model.) In Fig. 9 we show the energy spectrum of the fluxes from the five $p p$ chain reactions. In what follows we refer to the neutrino fluxes by the corresponding source reaction, so, for instance, the neutrinos produced from ${ }^{8} \mathrm{~B}$ decay are called ${ }^{8} \mathrm{~B}$ neutrinos. Most reactions produce a neutrino spectrum characteristic of $\beta$ decay. For ${ }^{8} \mathrm{~B}$ neutrinos the energy distribution presents deviations with respect to the maximum allowed energy be-

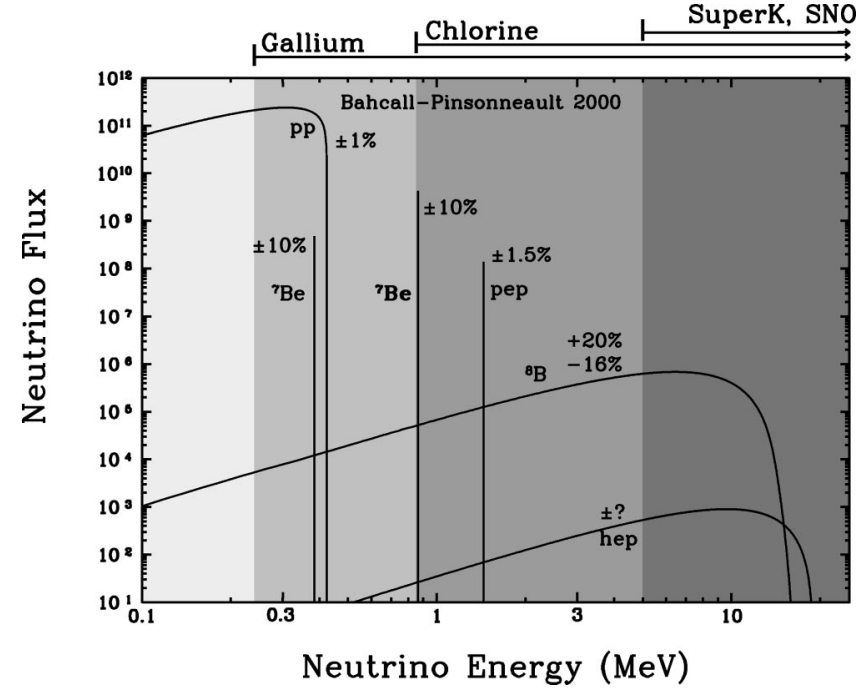

FIG. 9. Neutrino fluxes from the $p p$ chain reactions as a function of neutrino energy.

cause the final state, ${ }^{8} \mathrm{Be}$, is a wide resonance. On the other hand, the ${ }^{7} \mathrm{Be}$ neutrinos are almost monochromatic, with an energy width of about $2 \mathrm{keV}$, which is characteristic of the temperature in the core of the Sun.

As discussed in Sec. III, to describe the evolution of neutrinos in solar matter, one needs to know other quantities that are predicted by the SSM, such as the density and composition of solar matter, which give the solar electron number density. As discussed in Sec. III and shown in Fig. 5, the density of solar matter decreases monotonically and can be approximated by an exponential profile. Furthermore, in order to determine precisely the evolution of the neutrino system one also needs to know the production point distribution for the different neutrino fluxes. In Fig. 10 we show the prediction of Bahcall, Pinsonneault, and Basu (2001) for the distribution probability of the $p p$ chain.

The standard solar models have had notable successes. In particular, the comparison between the observed and the theoretically predicted sound speeds and

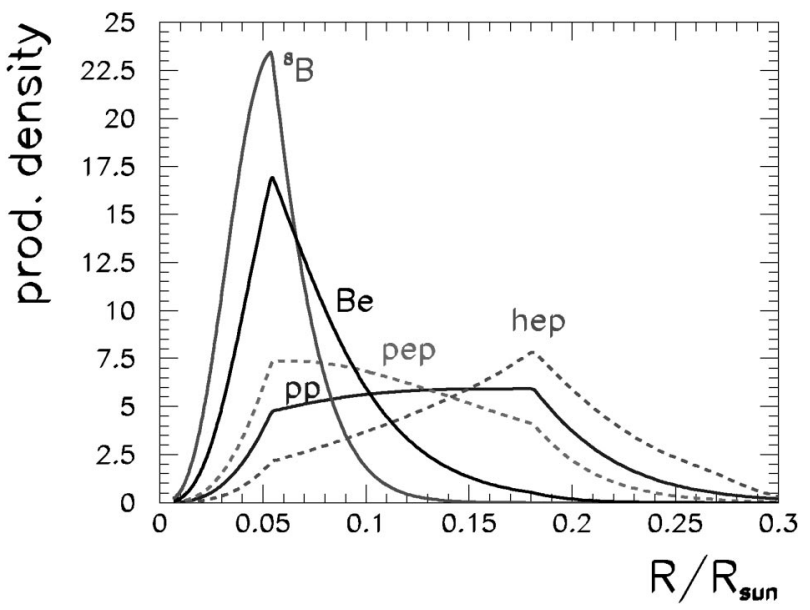

FIG. 10. Production-point distribution of $p p$ chain neutrinos as a function of the distance from the solar center. 
pressures has tested the SSM and provided accurate information on the solar interior. The solar quantities that have been determined by helioseismology include the sound velocity and matter density as a function of solar radius, the depth of the convective zone, the interior rotation rate, and the surface helium abundance. The excellent agreement between helioseismological observations and solar model calculations has made a convincing case that the large discrepancies between solar neutrino measurements and solar model predictions cannot be due to errors in the solar models.

\section{A. Solar neutrino experiments}

\section{Chlorine experiment: Homestake}

The first result on the detection of solar neutrinos was announced by Ray Davis, Jr. and his collaborators from Brookhaven in 1968 (Davis, Harmer, and Hoffman, 1968). In the Homestake gold mine in Lead, South Dakota, they installed a detector consisting of $\sim 615$ tons of $\mathrm{C}_{2} \mathrm{Cl}_{4}$. Solar $\nu_{e}$ 's are captured via

${ }^{37} \mathrm{Cl}\left(\nu, \mathrm{e}^{-}\right){ }^{37} \mathrm{Ar}$.

The energy threshold for this reaction is $0.814 \mathrm{MeV}$, so the relevant fluxes are the ${ }^{7} \mathrm{Be}$ and ${ }^{8} \mathrm{~B}$ neutrinos. ${ }^{7} \mathrm{Be}$ neutrinos excite the Gamow-Teller transition to the ground state with strength known from the lifetime of the electronic capture of ${ }^{37} \mathrm{Ar} .{ }^{8} \mathrm{~B}$ neutrinos can excite higher states of ${ }^{37} \mathrm{Ar}$, including the dominant transition to the isobaric state with transition energy $4.99 \mathrm{MeV}$. The strengths of the Gamow-Teller transitions to these excited states are determined from the isospin-related $\beta$ decay, ${ }^{37} \mathrm{Ca}\left(\beta^{+}\right){ }^{37} \mathrm{~K}$. For the SSM fluxes, $78 \%$ of the expected number of events are due to ${ }^{8} \mathrm{~B}$ neutrinos while $13 \%$ arise from ${ }^{7} \mathrm{Be}$ neutrinos. The produced ${ }^{37} \mathrm{Ar}$ was extracted radiochemically approximately every three months, and the number of ${ }^{37} \mathrm{Ar}$ decays $\left(t_{1 / 2}\right.$ $=34.8$ days) measured in a proportional counter.

The average event rate measured during the more than 20 years of operation is

$$
R_{\mathrm{Cl}}=2.56 \pm 0.16 \pm 0.16 \mathrm{SNU} \text {, }
$$

where $1 \mathrm{SNU}=10^{-36}$ captures/atom/sec. This corresponds to approximately one third of the SSM prediction (for the latest publications, see Cleveland et al., 1998, and Lande et al., 1999).

\section{Gallium experiments: SAGE and GALLEX/GNO}

In January 1990 and May 1991, two new radiochemical experiments using a ${ }^{71} \mathrm{Ga}$ target started taking data, SAGE and GALLEX. The SAGE detector is located in Baksan, Kaberdino-Balkaria, Russia, with 30 tons (increased to 57 tons from July 1991) of liquid metallic Ga. GALLEX is located in Gran Sasso, Italy, and consists of 30 tons of $\mathrm{GaCl}_{3}-\mathrm{HCl}$. In these experiments the solar neutrinos are captured via

${ }^{71} \mathrm{Ga}\left(\nu, e^{-}\right){ }^{71} \mathrm{Ge}$.
The special properties of this target include a low threshold $(0.233 \mathrm{MeV})$ and a strong transition to the ground level of ${ }^{71} \mathrm{Ge}$, which gives a large cross section for the lower-energy $p p$ neutrinos. According to the SSM, approximately $54 \%$ of the events are due to $p p$ neutrinos, while $26 \%$ and $11 \%$ arise from ${ }^{7} \mathrm{Be}$ and ${ }^{8} \mathrm{~B}$ neutrinos, respectively. The extraction of ${ }^{71} \mathrm{Ge}$ takes place every 3-4 weeks and the number of ${ }^{71} \mathrm{Ge}$ decays $\left(t_{1 / 2}=11.4\right.$ days $)$ is measured in a proportional counter.

The event rates measured by SAGE (Abdurashitov et al., 1999, 2002) and GALLEX (Hampel et al., 1999) are

$$
\begin{aligned}
& R_{\mathrm{SAGE}}=70.8_{-5.2-3.2}^{+5.3+3.7} \mathrm{SNU}, \\
& R_{\mathrm{GALLEX}}=77.5 \pm 6.2_{-4.7}^{+4.3} \mathrm{SNU},
\end{aligned}
$$

while the prediction of the SSM is 130 SNU.

The GALLEX program was completed in fall of 1997 and its successor, GNO, started taking data in spring of 1998. The latest combined GALLEX/GNO result is (Kirsten, 2002)

$$
R_{\mathrm{GALLEX}+\mathrm{GNO}}=70.8 \pm 5.9 \mathrm{SNU} \text {. }
$$

Since the $p p$ flux is directly constrained by the solar luminosity, in all stationary solar models there is a theoretical minimum of the expected number of events of 79 SNU. Furthermore, the largest uncertainties on the capture cross section for ${ }^{7} \mathrm{Be}$ neutrinos were considerably reduced after direct calibration using neutrinos from ${ }^{51} \mathrm{Cr}$ decay as a source.

\section{Water Cerenkov experiments: Kamiokande and SuperKamiokande}

Kamiokande and its successor SuperKamiokande (SK) in Japan are water Cerenkov detectors that are able to detect in real time the electrons scattered from the water by elastic interaction of the solar neutrinos,

$$
\nu_{a}+e^{-} \rightarrow \nu_{a}+e^{-} .
$$

The scattered electrons produce Cerenkov light, which is detected by photomultipliers. Notice that, while the detection process in radiochemical experiments is purely a charged-current ( $W$-exchange) interaction, the detection process of Eq. (84) goes through both charged- and neutral-current ( $Z$-exchange) interactions. Consequently, the detection process (84) is sensitive to all active neutrino flavors, although $\nu_{e}$ 's (which are the only ones to scatter via $W$ exchange) give a contribution that is about six times larger than that of $\nu_{\mu}$ 's or $\nu_{\tau}$ 's.

Kamiokande, with 2140 tons of water, started taking data in January 1987 and was terminated in February 1995. SK, with 45000 tons of water (of which 22500 are usable in solar neutrino measurements) started in May 1996, and it has analyzed so far events corresponding to 1258 days. The detection threshold in Kamiokande was $7.5 \mathrm{MeV}$ while SK started at a $6.5-\mathrm{MeV}$ threshold and is currently running at $5 \mathrm{MeV}$. This means that these experiments are able to measure only the ${ }^{8} \mathrm{~B}$ neutrinos (and the very small hep neutrino flux). Their results are presented in terms of measured ${ }^{8} \mathrm{~B}$ flux. 


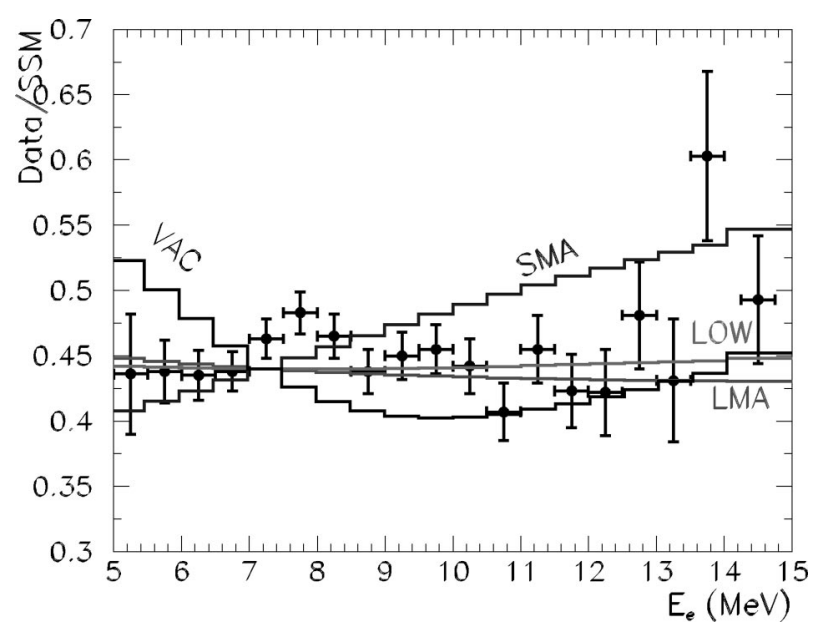

FIG. 11. The electron recoil energy spectrum measured at SuperKamiokande normalized to the Standard Solar Model prediction, and the expectations for the best-fit points for the solutions from Fig. 16 below: LMA, large mixing angle; SMA, small mixing angle; LOW, low mass ratio; VAC, vacuum oscillations.

The final result of Kamiokande (Fukuda et al., 1996) and the latest result of SK (Smy et al., 2002) are

$$
\begin{aligned}
& \Phi_{\mathrm{Kam}}=(2.80 \pm 0.19 \pm 0.33) \times 10^{6} \mathrm{~cm}^{-2} \mathrm{~s}^{-1}, \\
& \Phi_{\mathrm{SK}}=(2.35 \pm 0.02 \pm 0.08) \times 10^{6} \mathrm{~cm}^{-2} \mathrm{~s}^{-1}
\end{aligned}
$$

corresponding to about $40-50 \%$ of the SSM prediction.

There are three features unique to the water Cerenkov detectors. First, they are real-time experiments. Each event is individually recorded. Second, for each event the scattered electron keeps the neutrino direction within an angular interval which depends on the neutrino energy as $\sqrt{2 m_{e} / E_{\nu}}$. Thus it is possible, for example, to correlate the neutrino detection with the position of the Sun. Third, the amount of Cerenkov light produced by the scattered electron allows a measurement of its energy. In summary, the experiment provides information on the time, direction, and energy for each event. As we discuss below, signatures of neutrino oscillations might include distortion of the recoil electron energy spectrum, a difference between nighttime and daytime solar neutrino fluxes, or a seasonal variation in the neutrino flux. Observation of these effects would be strong evidence in support of solar neutrino oscillations independent of absolute flux calculations. Conversely, nonobservation of these effects could constrain oscillation solutions to the solar neutrino problem.

Over the years, the SK collaboration has presented information on the energy and time dependence of their event rates in different forms. In Fig. 11 we show their spectrum, corresponding to 1258 days of data, relative to the predicted spectrum (Ortiz, et al., 2000) normalized to the prediction of Bahcall, Pinsonneault, and Basu (2001). As seen from the figure, no significant distortion is observed and the data are compatible with a horizontal straight line.

The SK collaboration has also measured the event rates as a function of the time of day or night or, in other

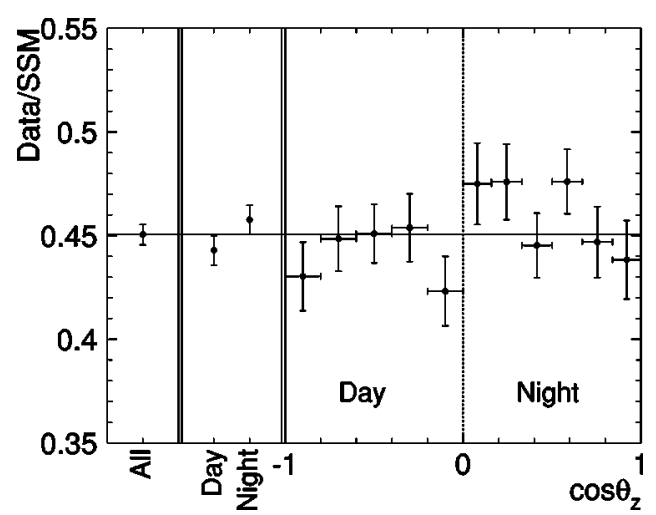

FIG. 12. The zenith-angle dependence of the solar neutrino flux (statistical error only). The width of the nighttime bins was chosen to separate solar neutrinos that pass through the Earth's dense core $\left(\cos \theta_{z} \geqslant 0.84\right)$ from those that pass through the mantle $\left(0<\cos \theta_{z}<0.84\right)$. The horizontal line shows the average flux.

words, of the position of the Sun in the sky. The results are presented as a nadir angle (the angle the Sun forms with the vertical) distribution of events. In Fig. 12 we show their nadir angle distribution, corresponding to 1258 days of data. SK have also presented their results on the day-night variation in the form of a day-night asymmetry,

$$
A_{N-D} \equiv 2 \frac{N-D}{D+N}=0.021 \pm 0.020 \text { (stat.) } \pm 0.013 \text { (syst.), }
$$

where $D(N)$ is the event rate during the day (night) period. The SK results show a small excess of events during the night but only at the $0.8 \sigma$ level.

In order to simultaneously account for the energy and day-night variation, SK present the observed energy spectrum during the day and during the night separately as well as in several night bins. This is the most convenient observable for including both time and energy dependence simultaneously in the analysis. The reason for that is that the separate distributions-energy spectrum averaged in time and time dependence averaged in energy — do not correspond to independent data samples and therefore have nontrivial correlations.

Finally, the SK collaboration has also measured the seasonal dependence of the solar neutrino flux. Their results after 1258 days of data are shown in Fig. 13. The points represent the measured flux, and the curve shows the expected variation due to the orbital eccentricity of the Earth (assuming no new physics, and normalized to the measured total flux). As can be seen from the figure, the data are consistent with the expected annual variation.

\section{SNO}

The Sudbury Neutrino Observatory (SNO) was first proposed in 1987 and it started taking data in November 1999 (McDonald et al., 2000). The detector, a great sphere surrounded by photomultipliers, contains ap- 


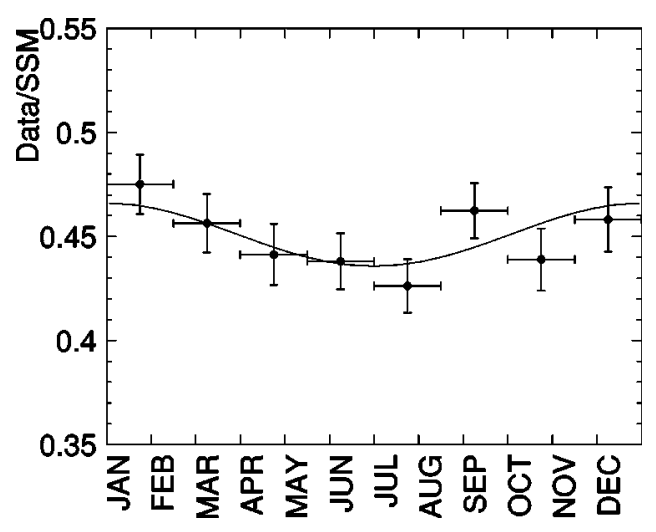

FIG. 13. Seasonal variation of the solar neutrino flux (statistical errors only). The curve shows the expected seasonal variation of the flux induced by the eccentricity of the Earth's orbit.

proximately 1000 tons of heavy water, $\mathrm{D}_{2} \mathrm{O}$, and is located at the Creighton mine, near Sudbury in Canada. It is immersed in ultrapure light water to provide shielding from radioactivity. SNO was designed to give a modelindependent test of the possible explanations of the observed deficit in the solar neutrino flux by having sensitivity to all flavors of active neutrinos and not just to $\nu_{e}$.

This sensitivity is achieved because energetic neutrinos can interact in the $\mathrm{D}_{2} \mathrm{O}$ of $\mathrm{SNO}$ via three different reactions. Electron neutrinos may interact via the charged-current reaction

$$
\nu_{e}+d \rightarrow p+p+e^{-}
$$

and can be detected above an energy threshold of a few $\mathrm{MeV}$ (presently $T_{e}>5 \mathrm{MeV}$ ). All active neutrinos $\left(\nu_{a}\right.$ $\left.=\nu_{e}, \nu_{\mu}, \nu_{\tau}\right)$ interact via the neutral-current reaction

$$
\nu_{a}+d \rightarrow n+p+\nu_{a},
$$

with an energy threshold of $2.225 \mathrm{MeV}$. Nonsterile neutrinos can also interact via elastic scattering, $\nu_{a}+e^{-}$ $\rightarrow \nu_{a}+e^{-}$, but with a smaller cross section.

The experimental plan of SNO consists of three phases, of approximately one year duration each. In its first year of operation, SNO has concentrated on the measurement of the charged-current reaction rate, while in a following phase, after the addition of $\mathrm{MgCl}_{2}$ salt to enhance the neutral-current signal, it will also perform a precise measurement of the neutral-current rate. In the third phase, the salt will be eliminated and a network of proportional counters filled with ${ }^{3} \mathrm{He}$ will be added with the purpose of directly measuring the neutral-current rate $\left.{ }^{3} \mathrm{He}(n, p)\right)^{3} \mathrm{H}$.

SNO can also perform measurements of the energy spectrum and time variation of the event rates. But the uniqueness of SNO lies in its ability to test directly whether the deficit of solar $\nu_{e}$ is due to changes in the flavor composition of the solar neutrino beam, since the charged-current/neutral-current ratio compares the number of $\nu_{e}$ interactions with those from all active flavors. This comparison is independent of the overall flux normalization.

In June 2001, SNO published their first results (Ahmad, 2001) on the charged-current measurement, and in
April 2002 they published their results from the first phase of the experiment, which included the day-night spectrum data in the full energy range above a threshold $T_{e}>5 \mathrm{MeV}$. Their spectrum is the result of the combination of the three possible signals. Assuming an undistorted energy spectrum, they extract the individual rates

$$
\begin{aligned}
& \Phi_{\mathrm{SNO}}^{C C}=\left(1.76_{-0.05}^{+0.06} \pm 0.09\right) \times 10^{6} \mathrm{~cm}^{-2} \mathrm{~s}^{-1} \\
& \Phi_{\mathrm{SNO}}^{E S}=(2.39 \pm 0.24 \pm 0.12) \times 10^{6} \mathrm{~cm}^{-2} \mathrm{~s}^{-1}, \\
& \Phi_{\mathrm{SNO}}^{N C}=\left(5.09_{-0.43-0.43}^{+0.44+0.46}\right) \times 10^{6} \mathrm{~cm}^{-2} \mathrm{~s}^{-1},
\end{aligned}
$$

and a day-night asymmetry $A_{N-D}=0.07 \pm 0.049$ (stat.) \pm 0.013 (syst.).

Notice that the neutral-current measured flux $\Phi_{\mathrm{SNO}}^{N C}$ is in excellent agreement with the prediction of the SSM, $\Phi_{{ }_{\mathrm{B}}}^{\mathrm{BP} 00}=\left(5.05_{-0.16}^{+0.20}\right) \times 10^{6} \mathrm{~cm}^{-2} \mathrm{~s}^{-1}$, which confirms that the observed flux of active neutrinos is consistent with the SSM.

\section{Future: Borexino and low-energy experiments}

The Borexino experiment (Oberauer, 1999) is designed to detect low-energy solar neutrinos in real time through the observation of the elastic-scattering process $\nu_{a}+e^{-} \rightarrow \nu_{a}+e^{-}$. The energy threshold for the recoil electrons is $250 \mathrm{keV}$. It will use 300 tons of liquid scintillator in an unsegmented detector with 2000 photomultiplier tubes. The event rate predicted by the SSM for a fiducial volume of about 100 tons is about 50 events per day, mostly generated by the $0.86-\mathrm{MeV}$ monoenergetic line of ${ }^{7} \mathrm{Be}$ solar neutrinos. Since this line gives a characteristic spectral signature in the elastic-scattering process, the flux on the earth of ${ }^{7} \mathrm{Be}$ solar neutrinos will be determined and it will be possible to check whether it is suppressed with respect to the flux predicted by the SSM, as is suggested by the results of current experiments. The Borexino experiment is under construction in the Laboratori Nazionali del Gran Sasso in Italy and is scheduled to start data taking in the near future.

A new generation of experiments aiming at a highprecision real-time measurement of the low-energy solar neutrino spectrum is now under study (Lanou et al., 1993; Arpesella, Broggini, and Cattadori, 1996; Raghavan, 1997; de Bellfon, 1999). Some of them, such as HELLAZ, HERON, and SUPER-MuNu, intend to detect the elastic scattering of electron neutrinos with the electrons of a gas and measure the recoil electron energy and its direction of emission. The proposed experiment LENS plans to detect the electron neutrino via its absorption in a heavy nuclear target, with the subsequent emission of an electron and a delayed gamma emission. The expected rates of these experiments for the proposed detector sizes are of the order of $\sim 1-10 \mathrm{pp}$ neutrinos a day. Consequently, with a running time of two years, they can reach a sensitivity of a few percent in the total neutrino rate at low energy, provided that they can achieve sufficient background rejection. 
TABLE II. Standard solar model predictions: solar neutrino fluxes and neutrino capture rates for the different experiments, with $1 \sigma$ uncertainties.

\begin{tabular}{lccccc}
\hline \hline Source & \multicolumn{1}{c}{$\begin{array}{c}\text { Flux } \\
\left(10^{10} \mathrm{~cm}^{-2} \mathrm{~s}^{-1}\right)\end{array}$} & $\begin{array}{c}\mathrm{Cl} \\
(\mathrm{SNU})\end{array}$ & $\begin{array}{c}\mathrm{Ga} \\
(\mathrm{SNU})\end{array}$ & $\begin{array}{c}\mathrm{SK} \\
\left(10^{6} \mathrm{~cm}^{-2} \mathrm{~s}^{-1}\right)\end{array}$ & $\begin{array}{c}\text { SNO(CC) SNO(NC) } \\
\left(10^{6} \mathrm{~cm}^{-2} \mathrm{~s}^{-1}\right)\end{array}$ \\
\hline pp & $5.95\left(1.00_{-0.01}^{+0.01}\right)$ & 0.0 & 69.7 & 0.0 & 0.0 \\
pep & $1.40 \times 10^{-2}\left(1.00_{-0.015}^{+0.015}\right)$ & 0.22 & 2.8 & 0.0 & 0.0 \\
hep & $9.3 \times 10^{-7}$ & 0.04 & 0.1 & 0.0093 & 0.0093 \\
${ }^{7} \mathrm{Be}$ & $4.77 \times 10^{-1}\left(1.00_{-0.10}^{+0.10}\right)$ & 1.15 & 34.2 & 0.0 & 0.0 \\
${ }^{8} \mathrm{~B}$ & $5.05 \times 10^{-4}\left(1.00_{-0.16}^{+0.20}\right)$ & 6.76 & 12.2 & 5.05 & 5.05 \\
${ }^{13} \mathrm{~N}$ & $5.48 \times 10^{-2}\left(1.00_{-0.17}^{+0.21}\right)$ & 0.09 & 3.4 & 0.0 & 0.0 \\
${ }^{15} \mathrm{O}$ & $4.80 \times 10^{-2}\left(1.00_{-0.19}^{+0.25}\right)$ & 0.33 & 5.5 & 0.0 & 0.0 \\
${ }^{17} \mathrm{~F}$ & $5.63 \times 10^{-4}\left(1.00_{-0.25}^{+0.25}\right)$ & 0.0 & 0.1 & 0.0 & 0.0 \\
Total & & $7.66_{-1.1}^{+1.3}$ & $128_{-7}^{+9}$ & $5.05_{-0.81}^{+1.01}$ & $5.05_{-0.81}^{+1.01}$ \\
Measured & & $2.56 \pm 0.226$ & $70.8 \pm 4.3$ & $2.35 \pm 0.083$ & $1.76 \pm 0.115 .09 \pm 0.64$ \\
Measured & & $0.337 \pm 0.065$ & $0.55 \pm 0.048$ & $0.465 \pm 0.094$ & $0.348 \pm 0.0731 .01 \pm 0.23$ \\
\multicolumn{2}{c}{ SSM }
\end{tabular}

\section{B. The solar neutrino problem}

Table II summarizes our present knowledge of the solar neutrino fluxes, their contribution to the expected rates, and the data from measurements of solar neutrino experiments. The predicted event rates are linear functions of the seven important neutrino fluxes: $p-p$, $p e p$, hep, ${ }^{7} \mathrm{Be},{ }^{8} \mathrm{~B},{ }^{13} \mathrm{~N}$, and ${ }^{15} \mathrm{O}$. We can make the following statements (see the last row of the table):

- Before the neutral-current measurement at SNO, all experiments observed a flux that was smaller than the SSM predictions, $\Phi^{\mathrm{obs}} / \Phi^{\mathrm{SSM}} \sim 0.3-0.6$.

- The deficit is not the same for the various experiments, which may indicate that the effect is energy dependent.

These two statements constitute the solar neutrino problem (Bahcall, Bahcall, and Shaviv, 1968; Bahcall and Davis, 1976). More quantitatively, a fit to the data using the model of Bahcall, Pinsonneault, and Basu (2001) shows a disagreement of $6.7 \sigma$, which means that the probability of explaining this result as a consequence of a statistical fluctuation of standard particle physics is $P$ $=3 \times 10^{-11}$ (we follow here the latest discussion by Bahcall, 2002a).

One may wonder about the model dependence of the solar neutrino problem. To answer this question one can allow all the solar neutrino fluxes, with undistorted energy spectra, to be free parameters in fitting the measured solar neutrino event rates, subject only to the condition that the total observed luminosity of the Sun is produced by nuclear fusion. This luminosity constraint can be written as a convenient linear equation in the neutrino fluxes:

$$
1=\sum_{i}\left(\frac{\alpha_{i}}{10 \mathrm{MeV}}\right)\left(\frac{\phi_{i}}{8.532 \times 10^{10} \mathrm{~cm}^{-2} \mathrm{~s}^{-1}}\right),
$$

where $\phi_{i}$ are the individual neutrino fluxes ( $i=p p$, pep, hep, ${ }^{7} \mathrm{Be},{ }^{8} \mathrm{~B},{ }^{13} \mathrm{~N}$, and ${ }^{15} \mathrm{O}$ ) and $\alpha_{i}$ is the luminous energy released in the corresponding reaction (the most recent determination of these parameters can be found in Bahcall, 2002b). Since there are at present six experiments, one cannot use all seven of the neutrino fluxes as free parameters, and some additional constraints must be used. For instance, the ratio of the $p$ ep to $p p$ fluxes is usually taken to be the same as in the SSM. Also, the $\mathrm{CNO}$ nuclear reactions are assumed to be in equilibrium, and the ${ }^{13} \mathrm{~N}$ and ${ }^{15} \mathrm{O}$ neutrino fluxes are taken to be exactly equal (or, in some analyses, both are taken to be zero). Finally the contribution from hep neutrinos is usually neglected.

A fit of this type (Bahcall, 2002b) shows that, previous to the SNO measurement, the best fit always corresponded to an unphysical, negative ${ }^{7}$ Be flux. Forcing all fluxes to be positive, the hypothesis of no new physics was rejected at the effective $2.5 \sigma$ level (99\% C.L.). Furthermore, it required that ${ }^{7} \mathrm{Be}$ neutrinos be entirely missing, a result which many authors have argued is not physically or astrophysically reasonable (for instance, Bahcall and Bethe, 1990; Hata, Bludman, and Langacker, 1994; Parke, 1995; Heeger and Robertson, 1996; Bahcall, Krastev, and Smirnov, 1998).

The results of SNO have provided further modelindependent evidence on the problem. Both SNO and SK are sensitive mainly to the ${ }^{8} \mathrm{~B}$ flux. Without new physics, the measured fluxes in any reaction at these two experiments should be equal. Villante, Fiorentini, and Lisi (1999) showed how one can choose the energy thresholds for the SK and SNO experiments in such a way that the response functions for the two experiments are made approximately equal (see also Fogli, Lisi, Montanino, and Palazzo, 2001). The advantage of this method is that some of the systematic errors are reduced, but there is a slight loss of statistical power (Bahcall, 2002a; Berezinski, 2001). The first reported SNO charged-current result compared with the elasticscattering rate from SK showed that the hypothesis of no flavor conversion was excluded at $\sim 3 \sigma$.

Finally, with the neutral-current measurement at SNO one finds that 


$$
\begin{aligned}
\phi_{\mu \tau}^{\mathrm{SNO}} & \equiv \phi_{{ }_{8} \mathrm{~B}}^{\mathrm{NC}, \mathrm{SNO}}-\phi_{{ }_{8}}^{\mathrm{CC}, \mathrm{SNO}} \\
& =\left(3.41 \pm 0.45_{-0.45}^{+0.48}\right) \times 10^{6} \mathrm{~cm}^{-2} \mathrm{~s}^{-1} .
\end{aligned}
$$

This result provides evidence for neutrino flavor transition (from $\nu_{e}$ to $\nu_{\mu, \tau}$ ) at the level of $5.3 \sigma$. This evidence is independent of the solar model.

\section{Solar neutrino oscillation probabilities}

The most generic and popular explanation of the solar neutrino anomaly is given by the introduction of neutrino masses and mixing, leading to oscillations of $\nu_{e}$ into an active $\left(\nu_{\mu}\right.$ and/or $\left.\nu_{\tau}\right)$ or a sterile $\left(\nu_{s}\right)$ neutrino. We now discuss some issues that have been raised in the literature concerning the computation of the corresponding neutrino survival probabilities in the full range of mass and mixing relevant to the solar neutrino problem.

\section{Quasivacuum oscillations and the dark side}

The presence of neutrino mass and mixing implies the possibility of neutrino oscillations. Solar electron neutrinos can undergo oscillations either in vacuum or via the matter-enhanced MSW mechanism, depending on the actual values of mass-squared differences and mixing angles. However, this simplified picture of solar neutrino oscillations contains a set of approximations that are not always valid in the context of solar neutrinos. In order to clarify this issue let us first review the calculation of the solar neutrino survival probability in the two-neutrino case.

The survival amplitude for a solar $\nu_{e}$ of energy $E$ at a terrestrial detector can be written as follows:

$$
A_{e e}=\sum_{i=1}^{2} A_{e i}^{S} A_{i e}^{E} \exp \left[-i m_{i}^{2}(L-r) /(2 E)\right] .
$$

Here $A_{e i}^{S}$ is the amplitude of the transition $\nu_{e} \rightarrow \nu_{i}$ from the production point to the surface of the Sun, and $A_{i e}^{E}$ is the amplitude of the transition $\nu_{i} \rightarrow \nu_{e}$ from the surface of the Earth to the detector. The propagation amplitude in vacuum from the surface of the Sun to the surface of the Earth is given by the exponential term, where $L$ is the distance between the center of the Sun and the surface of the Earth, and $r$ is the radius of the Sun. The corresponding survival probability $P_{e e}$ is then given by

$$
P_{e e}=P_{1} P_{1 e}+P_{2} P_{2 e}+2 \sqrt{P_{1} P_{2} P_{1 e} P_{2 e}} \cos \xi .
$$

Here $P_{i} \equiv\left|A_{e i}^{S}\right|^{2}$ is the probability that the solar neutrinos reach the surface of the Sun as $\left|\nu_{i}\right\rangle$, while $P_{i e}$ $\equiv\left|A_{i e}^{E}\right|^{2}$ is the probability of $\nu_{i}$ arriving at the surface of the Earth to be detected as a $\nu_{e}$. Unitarity implies $P_{1}$ $+P_{2}=1$ and $P_{1 e}+P_{2 e}=1$. The phase $\xi$ is given by

$$
\xi=\frac{\Delta m^{2}(L-r)}{2 E}+\delta,
$$

where $\delta$ contains the phases due to propagation in the Sun and in the Earth and can be safely neglected (an expression for this phase can be found in Lisi, Marrone, Montanino, Palazzo, and Petcov, 2001). In the evaluation of both $P_{i}$ and $P_{i e}$, the effect of coherent forward interaction with the matter of the Sun and the Earth must be taken into account.

From Eq. (91) one can recover more familiar expressions for $P_{e e}$ that hold in certain limits:

(1) For $\Delta m^{2} / E \lesssim 5 \times 10^{-17} \mathrm{eV}$, the matter effect suppresses flavor transitions in both the Sun and the Earth. Consequently, the probabilities $P_{1}$ and $P_{2 e}$ are simply the projections of the $\nu_{e}$ state onto the mass eigenstates: $P_{1}=\cos ^{2} \theta, P_{2 e}=\sin ^{2} \theta$. In this case we are left with the standard vacuum oscillation formula,

$$
P_{e e}^{\mathrm{vac}}=1-\sin ^{2} 2 \theta \sin ^{2}\left[\Delta m^{2}(L-r) / 4 E\right],
$$

which describes the oscillations on the way from the surface of the Sun to the surface of the Earth. The probability is symmetric under the change of octant, $\theta \leftrightarrow \pi / 2-\theta$, and change of sign $\Delta m^{2} \leftrightarrow-\Delta m^{2}$ (see Sec. III). This symmetry implies that each point in the $\left(\Delta m^{2}, \sin ^{2} 2 \theta\right)$ parameter space corresponds to two physically independent solutions, one in each octant.

Averaging Eq. (93) over the Earth's orbit, $L(t)$ $=L_{0}[1-\varepsilon \cos (2 \pi t / T)]$, one gets

$$
\begin{aligned}
\left\langle P_{e e}^{\mathrm{vac}}\right\rangle= & 1-\frac{1}{2} \sin ^{2} 2 \theta\left[1-\cos \left(\frac{\Delta m^{2} L_{0}}{2 E}\right)\right. \\
& \left.\times J_{0}\left(\frac{\varepsilon \Delta m^{2} L_{0}}{2 E}\right)\right],
\end{aligned}
$$

where $\varepsilon=0.0167$ is the orbit eccentricity and $J_{0}$ is the Bessel function. In Fig. 14(a) we display the value of $\left\langle P_{e e}^{\mathrm{vac}}\right\rangle$ as a function of $4 \mathrm{E} / \Delta \mathrm{m}^{2}$. As can be seen in the figure, for large values of $\Delta m^{2}$ the probability averages out to a constant value $1-\frac{1}{2} \sin ^{2}(2 \theta)$.

(2) For $\Delta m^{2} / E \gtrsim 10^{-14} \mathrm{eV}$, the last term in Eq. (91) vanishes and we recover the incoherent MSW survival probability. In this case, $P_{1}$ and $P_{2 e}$ must be obtained by solving the evolution equation of the neutrino states in the Sun and the Earth, respectively. As discussed in Sec. III.D, the approximate solution for the evolution in the Sun takes the well-known form

$$
P_{1}=\frac{1}{2}+\left(\frac{1}{2}-P_{L Z}\right) \cos 2 \theta_{m, 0} .
$$

Here $P_{L Z}$ denotes the standard Landau-Zener probability of Eq. (75) which, for the exponential profile, takes the form shown in Eq. (77), and $\theta_{m, 0}$ is the mixing angle in matter at the neutrino production point given in Eq. (55). For the approximation of an exponential density profile, $\gamma=\pi\left(\Delta m^{2} / E\right) r_{0}$, which is independent of the point in the Sun where the resonance takes place. Improvement over this constant-slope exponential density profile approximation can be obtained by numerically deriving the exact $N_{e}(r)$ profile at the resonant point. In this case $\gamma=\pi\left(\Delta m^{2} / E\right) r_{0}\left(r_{\text {res }}\right)$.

During the day there is no Earth-matter effect. The survival probabilities $P_{i e}$ are obtained by simple projec- 


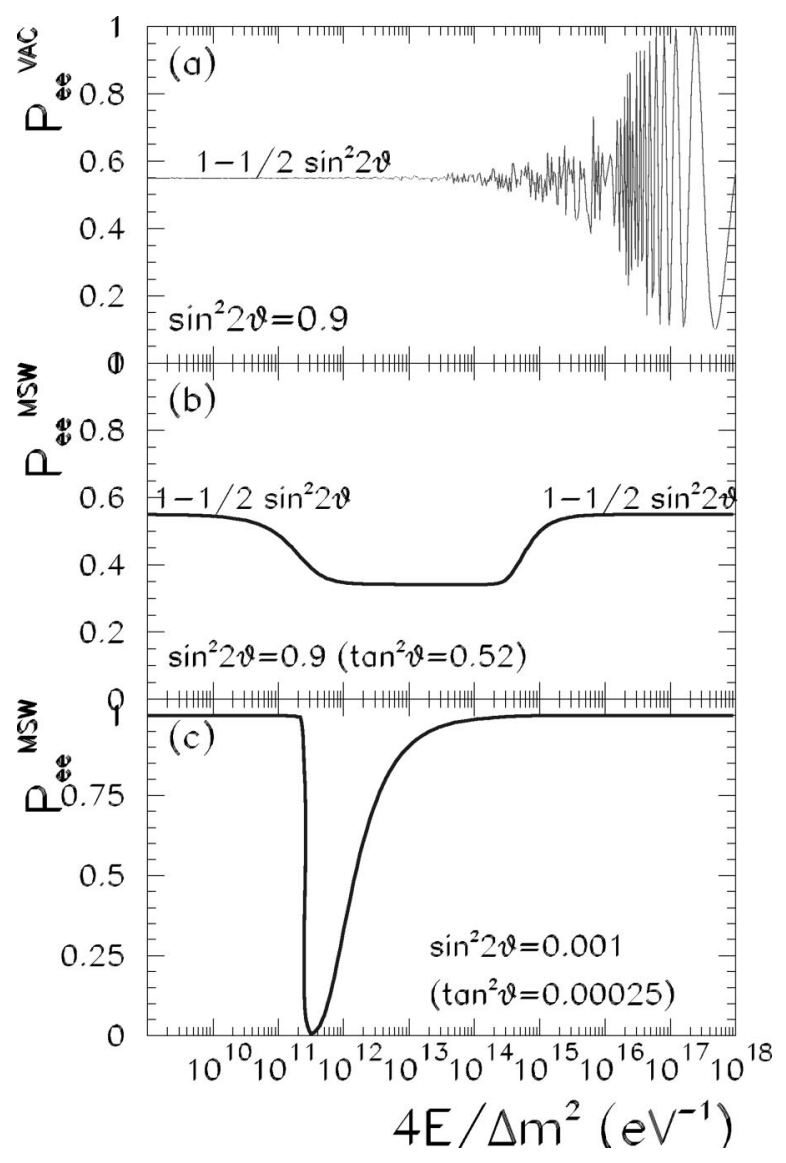

FIG. 14. $P_{e e}$ as a function of $4 E / \Delta m^{2}$. In panel (a) matter effects are ignored [see Eq. (94)]. Panels (b) (large mixing angle) and (c) (small mixing angle) take into account the MSW effect [see Eq. (96)].

tion of the $\nu_{e}$ state onto the mass eigenstates: $P_{2 e, D}=1$ $-P_{1 e, D}=\sin ^{2} \theta$. One obtains the expression in Eq. (76),

$$
P_{e e, D}^{\mathrm{MSW}}=\frac{1}{2}+\left(\frac{1}{2}-P_{L Z}\right) \cos 2 \theta_{m, 0} \cos 2 \theta .
$$

In Figs. 14(b) and (c) we plot the survival probability as a function of $4 E / \Delta m^{2}$ for a large and a small mixing angle, respectively.

Let us make some remarks concerning Eq. (96):

(i) In both the limits of large and very small $E / \Delta m^{2}$, $P_{e e}^{\mathrm{MSW}} \rightarrow 1-\frac{1}{2} \sin ^{2} 2 \theta$ [see Fig. 14(b)], which is the same expression as for averaged vacuum oscillations. This result, however, comes from very different reasons in the two regimes. For large $E / \Delta m^{2}, P_{L Z}=\cos ^{2} \theta$ and $\cos 2 \theta_{m, 0}=-1$. For small $E / \Delta m^{2}, P_{L Z}=0$ and $\cos 2 \theta_{m, 0}=\cos 2 \theta$.

(ii) Due to matter effects, $P_{e e}^{\mathrm{MSW}}$ is only symmetric under the simultaneous transformation $\left(\Delta m^{2}, \theta\right)$ $\rightarrow\left(-\Delta m^{2}, \pi / 2-\theta\right)$. For $\Delta m^{2}>0$, the resonance is only possible for $\theta<\pi / 4$, and MSW solutions are usually plotted in the $\left(\Delta m^{2}, \sin ^{2} 2 \theta\right)$ plane assuming that now each point on this plane represents only one physical solution with $\theta$ in the first octant. But in principle, nonresonant solutions are also possible for $\theta>\pi / 4$, the so-called dark side
(Fogli, Lisi, and Montanino, 1994, 1996; de Gouvea, Friedland, and Murayama, 2000; Fogli, Lisi, Montanino, and Palazzo, 2000a; Gonzalez-Garcia and Peña-Garay, 2000).

(iii) The analysis of the survival probability of solar neutrinos would be simplified if there were a region where $E / \Delta m^{2}$ were small enough that vacuum oscillations were averaged out and large enough to be in the extremely nonadiabatic MSW regime. This, however, is not the case, as can be seen from comparison of panels (a) and (b) of Fig. 14. In the intermediate range, $2 \times 10^{14} \leqslant 4 E / \Delta m^{2}$ $\lesssim 10^{16} \mathrm{eV}^{-1}$, adiabaticity is violated and the $\cos \xi$ coherent term should be taken into account. The result is similar to vacuum oscillations, but with small matter corrections. We define this case as quasivacuum oscillations (Petcov, 1988b; Petcov and Rich, 1989; Pakvasa and Pantaleone, 1990; Pantaleone, 1990; Fogli, Lisi, Montanino, and Palazzo 2000b; Friedland, 2000; Lisi, Marrone, Montanino, Palazzo, and Petcov, 2001). The range of $E / \Delta m^{2}$ for the quasivacuum oscillation regime depends on the value of $E / \Delta m^{2}$ for which the MSW probability in Eq. (96) acquires the asymptotic value $1-\frac{1}{2} \sin ^{2} 2 \theta$ : the smaller $E / \Delta m^{2}$, the more separated the MSW and vacuum regimes are, and the narrower the quasivacuum oscillation region.

It is clear from these considerations that in order to compute a survival probability for solar neutrinos that would be valid for any experiment and any value of the neutrino mass and mixing, one has to evaluate the full expression (91). The results that we show here were obtained using the general expression for the survival probability in Eq. (91), with $P_{1}$ and $P_{2 e}$ found by numerically solving the evolution equation for the Sun and the Earth. For the Sun, the evolution equation has to be solved from the neutrino production point to the edge of the Sun and averaged over the production point distribution shown in Fig. 10.

\section{Evolution in the Earth}

In this section we discuss matter effects in the Earth (for some of the original work see Bouchez et al., 1986; Baltz and Weneser, 1987, 1988; Mikheyev and Smirnov, 1987). The density of the Earth's matter, unlike that of the Sun, is not strongly varying. It consists mainly of two layers, the lower-density mantle and the higher-density core, each with approximately constant density. The core radius is $L=2896 \mathrm{~km}$.

When a neutrino crosses only the mantle, the effects of the Earth's matter are well approximated by evolution with a constant potential. In this case, the resulting probability simplifies to

$$
P_{2 e}=\sin ^{2} \theta+\frac{4 E V_{e}}{\Delta m^{2}} \sin ^{2} 2 \theta_{E} \sin ^{2} \frac{\pi L}{L_{m}},
$$


where $\theta_{E}$ is the mixing angle in the Earth [Eq. (55)], $L$ is the distance traveled by the neutrino within the Earth, and $L_{m}$ is the oscillation length in matter [Eq. (58)].

The right-hand side of Eq. (97) consists of two terms. The first term gives a simple projection of the mass to flavor state in vacuum (which corresponds to the probability during the daytime). The second is the regeneration term and will be denoted by $f_{\text {reg }}$ (for details see Gonzalez-Garcia, Peña-Garay, and Smirnov, 2001). It contains the Earth matter effects and it is always positive. Averaging out the distance-dependent term, we can now rewrite Eq. (91):

$$
P_{e e}^{N}=P_{e e}^{D}-\left(1-2 P_{L Z}\right) \cos 2 \theta_{m, 0} f_{\mathrm{reg}},
$$

where $P_{e e}^{D}$ and $P_{e e}^{N}$ are the $\nu_{e}$ survival probabilities during the day and night, respectively. Since $f_{\text {reg }}>0$ and, for the interesting cases of MSW transitions in the Sun, $\cos 2 \theta_{m, 0}<0$, the relative magnitude of $P_{e e}^{D}$ and $P_{e e}^{N}$ depends on the sign of $\left(1-2 P_{L Z}\right)$. For $P_{L Z}<0.5$ (and, in particular, for adiabatic MSW transitions, $\left.P_{L Z}=0\right)$ the survival probability during the night is larger than during the day. The opposite holds for large adiabaticity violations, $P_{L Z}>0.5$.

When neutrinos cross the core, adiabaticity may be violated in their evolution. The abrupt density change between the mantle and the core may induce a new form of resonance, different from the MSW resonance (Petcov, 1998; Akhmedov, 1999). This parametric resonance (Ermilova, Tsarev, and Chechin, 1986; Akhmedov, 1988) is relevant mainly for small mixing angles.

\section{Two-neutrino oscillation analysis}

\section{Predictions}

In an experiment $i$, the expected event rate in the presence of oscillations, $R_{i}^{\text {th }}$, can be written as follows:

$$
\begin{aligned}
R_{i}^{\mathrm{th}}= & \sum_{k=1,8} \phi_{k} \int d E_{\nu} \lambda_{k}\left(E_{\nu}\right) \times\left\{\sigma_{e, i}\left(E_{\nu}\right)\left\langle P_{e e}\left(E_{\nu}, t\right)\right\rangle\right. \\
& \left.+\sigma_{x, i}\left(E_{\nu}\right)\left[1-\left\langle P_{e e}\left(E_{\nu}, t\right)\right\rangle\right]\right\}
\end{aligned}
$$

where $E_{\nu}$ is the neutrino energy, $\phi_{k}$ is the total neutrino flux, and $\lambda_{k}$ is the neutrino energy spectrum (normalized to 1$)$ from the solar nuclear reaction $k$. Here $\sigma_{e, i}\left(\sigma_{x, i}\right)$ is the $\nu_{e}\left(\nu_{x}, x=\mu, \tau\right)$ interaction cross section in the standard model with the target corresponding to experiment $i$, and $\left\langle P_{e e}\left(E_{\nu}, t\right)\right\rangle$ is the time-averaged $\nu_{e}$ survival probability. The expected signal in the absence of oscillations, $R_{i}^{\mathrm{BP} 00}$, can be obtained from Eq. (99) by substituting $P_{e e}=1$. In Table II we give the expected rates at the different experiments which we obtain using the fluxes of Bahcall, Pinsonneault, and Basu (2001).

For the chlorine, gallium, and SNO (charged-current) measurements, only the electron neutrino contributes and the $\sigma_{x, i}$ term in Eq. (99) vanishes. For elastic scattering at $\mathrm{SK}$ or $\mathrm{SNO}$, there is a possible contribution from the neutral-current interaction of the other active neutrino flavors present in the beam. For the neutralcurrent rate at SNO, all active flavors contribute equally.
For absorption in chlorine and gallium, the cross sections $\sigma_{e, i}(E)$ can be found in Bahcall (1997). The cross sections for SNO and SK (elastic scattering) can be obtained from the differential cross sections of Nakamura et al. (2001) and Bahcall, Kamionkowsky, and Sirlin (1995), respectively, by integrating with the corresponding detector resolutions and for the given detection thresholds. In particular, for the SK and SNO energy spectrum data, to obtain $R^{\text {th }}$ in a given energy bin one has to integrate within the corresponding electron recoil energy bin and to take into account that finite energy resolution implies that the measured kinetic energy $T$ of the scattered electron is distributed around the true kinetic energy $T^{\prime}$ according to a resolution function $\operatorname{Res}\left(T, T^{\prime}\right)$ of the form (Bahcall, Krastev, and Lisi, 1997)

$$
\operatorname{Res}\left(T, T^{\prime}\right)=\frac{1}{\sqrt{2 \pi} s} \exp \left[-\frac{\left(T-T^{\prime}\right)^{2}}{2 s^{2}}\right],
$$

where $s=s_{0} \sqrt{T^{\prime} / \mathrm{MeV}}$, and $s_{0}=0.47 \mathrm{MeV}$ for SK (Faid et al., 1997). On the other hand, the distribution of the true kinetic energy $T^{\prime}$ for an interacting neutrino of energy $E_{\nu}$ is dictated by the differential cross section $d \sigma_{\alpha}\left(E_{\nu}, T^{\prime}\right) / d T^{\prime}$ and the kinematic limits are

$$
0 \leqslant T^{\prime} \leqslant \bar{T}^{\prime}\left(E_{\nu}\right)=\frac{E_{\nu}}{1+m_{e} / 2 E_{\nu}} .
$$

For assigned values of $s_{0}, T_{\min }$, and $T_{\max }$, the corrected cross section $\sigma_{a}(E)(a=e, x)$ is given as

$$
\begin{aligned}
\sigma_{a}\left(E_{\nu}\right)= & \int_{T_{\min }}^{T_{\max }} d T \int_{0}^{\bar{T}^{\prime}\left(E_{\nu}\right)} d T^{\prime} \\
& \times \operatorname{Res}\left(T, T^{\prime}\right) \frac{d \sigma_{\alpha}\left(E_{\nu}, T^{\prime}\right)}{d T^{\prime}} .
\end{aligned}
$$

For data taken within a given zenith angle period $i$, the expected number of events in the presence of oscillations is

$$
\begin{aligned}
R_{i}^{\mathrm{th}}= & \frac{1}{\Delta \tau_{i}} \int_{\tau\left(\cos \Phi_{\min , i}\right)}^{\tau\left(\cos \Phi_{\max , i}\right)} d \tau \sum_{k=1,8} \phi_{k} \int d E_{\nu} \lambda_{k}\left(E_{\nu}\right) \\
& \times\left\{\sigma_{e, i}\left(E_{\nu}\right)\left\langle P_{e e}\left(E_{\nu}, \tau\right)\right\rangle\right. \\
& \left.+\sigma_{x, i}\left(E_{\nu}\right)\left[1-\left\langle P_{e e}\left(E_{\nu}, \tau\right)\right\rangle\right]\right\},
\end{aligned}
$$

where $\tau$ measures the annual averaged length of the period $i$ normalized to $1: \Delta \tau_{i}=\tau\left(\cos \Phi_{\text {max }, i}\right)-\tau\left(\cos \Phi_{\min , i}\right)$. For instance, if the period $i$ corresponds to an entire day $(i=D)$ or night $(i=N)$ then $\Delta \tau_{D(N)}=0.5$. From these predictions one can easily build the corresponding expected day-night asymmetry. In Fig. 15 we show the isocontours of expected $A_{N-D}$ at SK in the $\left(\Delta m^{2}, \tan ^{2} \theta\right)$ plane for active neutrino oscillations (the results are very similar for sterile neutrinos). As discussed in Sec. IV.C, in most of the parameter space, the effect of traveling through the Earth is the regeneration of the $\nu_{e}$ component, resulting in a positive day-night asymmetry with the exception of the region where the nonadiabaticity of the oscillations in the Sun is important $\left(\tan ^{2} \theta\right.$ $\left.\sim 10^{-3}, \Delta m^{2} \sim 3 \times 10^{-6} \mathrm{eV}^{2}\right)$. As discussed above, SK 


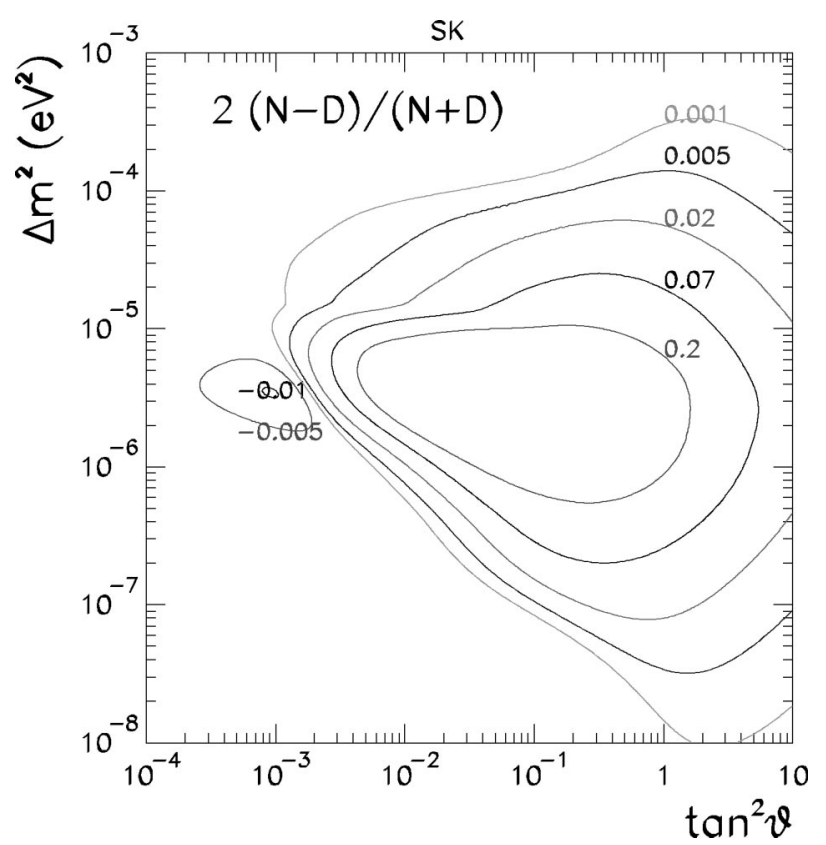

FIG. 15. Isocontours of the day-night asymmetry at SK.

has observed a very small day-night asymmetry. This observation implies that some of the oscillation parameter space can be excluded, as we discuss below.

In the same fashion, integrating Eq. (103) for the different electron recoil energy bins and for the day and night periods separately, one can obtain predictions for SK and SNO day-night spectra.

\section{Analysis of total event rates: allowed masses and mixing}

The goal of analyzing of the solar neutrino data in terms of neutrino oscillation is to determine which range of mass-squared difference and mixing angle is responsible for the observed deficit (see, for instance, Hata and Langacker, 1997; Bahcall, Krastev, and Smirnov, 1998; Fogli, Lisi, and Montanino, 1998; Gonzalez-Garcia, de Holanda, et al., 2000). In order to answer this question in a statistically meaningful way, one must compare the predictions in the different oscillation regimes with the observations, including all the sources of uncertainties and their correlations, by building, for instance, the $\chi^{2}$ function

$$
\chi_{R}^{2}=\sum_{i}\left(R_{i}^{\mathrm{th}}-R_{i}^{\mathrm{exp}}\right) \sigma_{i j}^{-2}\left(R_{j}^{\mathrm{th}}-R_{j}^{\exp }\right) .
$$

Here $R_{i}^{\text {th }}$ is the theoretical expectation (99), $R_{i}^{\exp }$ is the observed number of events in the experiment $i$, and $\sigma_{i j}$ is the error matrix, which contains both the theoretical uncertainties and the experimental statistical and systematic errors. The main sources of uncertainty are the theoretical errors in the prediction of solar neutrino fluxes for the different reactions. These errors are due to uncertainties in the 12 basic ingredients of the solar model, which include the nuclear reaction rates (parametrized in terms of the astrophysical factors $S_{11}, S_{33}$,

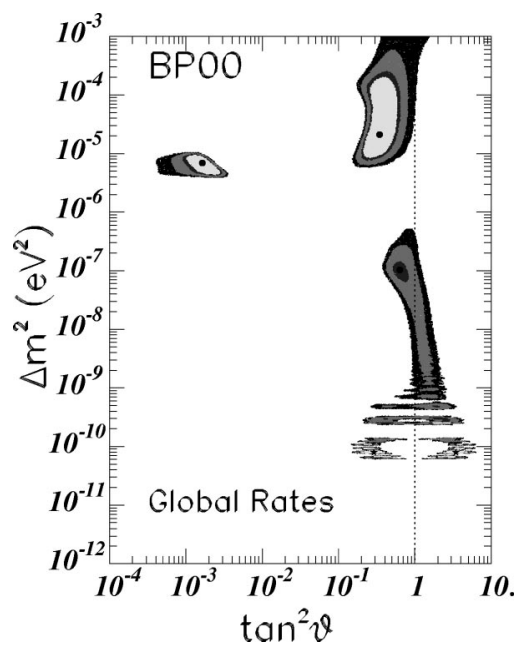

FIG. 16. Allowed oscillation parameters (at 90\%, 95\%, 99\%, and $99.7 \%$ C.L.) from the analysis of the total event rates of the chlorine, gallium, SK, and SNO charged-current experiments. The best-fit point is marked with a star.

$S_{34}, S_{1,14}$, and $S_{17}$ ), the solar luminosity, the metalicity $Z / X$, the Sun's age, the opacity, the diffusion, and the electronic capture of ${ }^{7} \mathrm{Be}, C_{\mathrm{Be}}$. These errors are strongly correlated as the same astrophysical factor can affect the different neutrino production rates. Another source of theoretical error arises from uncertainties in the neutrino interaction cross section for the different detection processes. For a detailed description of how to include all these uncertainties and correlations in the construction of $\sigma_{i j}$ we refer the reader to the work of Fogli and Lisi (1995a). Updated uncertainties can be found in Fogli, Lisi, Montanino, and Palazzo (2000a) and Gonzalez-Garcia and Peña-Garay (2001).

The results of the analysis of the total event rates are shown in Fig. 16, where we plot the allowed regions that correspond to $90 \%, 95 \%, 99 \%$, and $99.73 \%(3 \sigma)$ C.L. for $\nu_{e}$ oscillations into active neutrinos.

As can be seen in the figure, for oscillations into active neutrinos there are several oscillation regimes which are compatible within errors with the experimental data. These allowed parameter regions are labeled as $M S W$ small mixing angle (SMA), MSW large mixing angle (LMA), MSW low mass (LOW), and vacuum oscillations (VAC). Before including the SNO (chargedcurrent) data, the best fit corresponded to the SMA solution, but after SNO the best fit corresponds to the LMA solution. For the LMA solution, oscillations for the ${ }^{8} \mathrm{~B}$ neutrinos occur in the adiabatic regime, and the survival probability is higher for lower-energy neutrinos. This situation fits well with the higher rate observed at gallium experiments. For the LOW solution, the situation is opposite, but matter effects in the Earth for $p p$ and ${ }^{7} \mathrm{Be}$ neutrinos enhance the average annual survival probability for these lower-energy neutrinos. The combination of these effects still allows a reasonable description of the gallium rate.

Oscillations into pure sterile neutrinos are strongly 
disfavored by the SNO data since they would imply very similar neutrino fluxes for the neutral-current, chargedcurrent, and elastic-scattering rates in SNO as well as the elastic-scattering rate at SK. Schematically, in the presence of oscillations,

$$
\begin{aligned}
& \Phi^{\mathrm{CC}}=\Phi_{e}, \\
& \Phi^{\mathrm{ES}}=\Phi_{e}+r \Phi_{\mu \tau}, \\
& \Phi^{\mathrm{NC}}=\Phi_{e}+\Phi_{\mu \tau},
\end{aligned}
$$

where $r \equiv \sigma_{\mu} / \sigma_{e} \simeq 0.15$ is the ratio of the $\nu_{e}-e$ and $\nu_{\mu}$ $-e$ elastic scattering cross sections. The flux $\Phi_{\mu \tau}$ of active no-electron neutrinos in the beam would vanish if $\nu_{e}$ oscillate into a purely sterile state. Thus, if the beam comprises only $\nu_{e}$ 's and $\nu_{s}$ 's, the three observed rates should be equal (up to effects due to spectral distortions), a hypothesis that is now ruled out at more than $5 \sigma$ by the SNO data. Oscillations into an admixture of active and sterile states are still allowed provided that the ${ }^{8} \mathrm{~B}$ neutrino flux is allowed to be larger than the SSM expectation (Bahcall, Gonzalez-Garcia, and Peña-Garay, 2002b; Barger, Marfatia, and Whisnant, 2002).

\section{Day-night spectra: Excluded masses and mixing}

Further information on the different oscillation regimes can be obtained from the analysis of the energy and time dependence data from SK (for an early suggestion of the use of the day-night spectra see Maris and Petcov, 1997), which is currently presented in the form of the observed day-night or zenith spectrum (Fukuda et al., 2001: Smy et al., 2002). The way to treat these data statistically has been discussed, for instance, by Bahcall, Krastev, and Smirnov (2000), Gonzalez-Garcia, de Holanda, et al. (2000), Fukuda et al. (2001), and Gonzalez-Garcia and Peña-Garay (2001).

The observed day-night spectrum in SK is essentially undistorted in comparison to the SSM expectation and shows no significant differences between the day and the night periods. Consequently, a large region of the oscillation parameter space where these variations are expected to be large can be excluded. In Fig. 11 we show the SK spectrum corresponding to 1258 days of data relative to the Ortiz et al. (2000) spectrum normalized to the predictions of Bahcall, Pinsonneault, and Basu (2001), together with the expectations from the best-fit points for the large-mixing-angle, small-mixing-angle, low-mass, and vacuum oscillation solutions.

The various solutions give different predictions for the day-night spectrum. For large mixing angle and low mass, the expected spectrum is distorted very little. For small mixing angle, a positive slope is expected, with larger slope for larger mixing angle within the smallmixing-angle region. For vacuum oscillations, large distortions are expected. The details are dependent on the precise values of the oscillation parameters.

In Fig. 17 we show the excluded regions at the 99\% C.L. from the analysis of SK day-night spectrum data, together with the contours corresponding to the $95 \%$ and $99.73 \%(3 \sigma)$ C.L. In particular, the central region

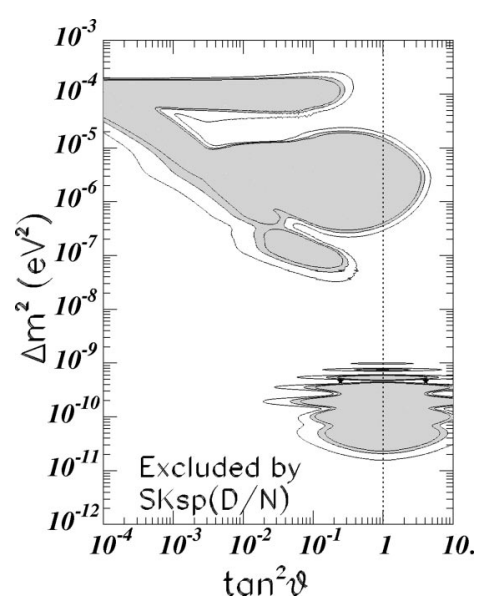

FIG. 17. Excluded oscillation parameters at 95\%, 99\% (shaded region), and $99.7 \%$ C.L. from the analysis of the daynight spectrum data.

$\left(2 \times 10^{-5}<\Delta m^{2}<3 \times 10^{-7}, \tan ^{2} \theta>3 \times 10^{-3}\right)$ is excluded due to the small observed day-night variation (compare to Fig. 15). The rest of the excluded region is due to the absence of any observed distortion of the energy spectrum.

Superimposing Figs. 17 and 16, we can deduce the main consequences of adding the day-night spectrum information to the analysis of the total event rates:

- SMA: within this region, the part with larger mixing angle fails to comply with the observed energy spectrum, while the part with smaller mixing angles gives a bad fit to the total rates.

- VAC: the observed flat spectrum cannot be accommodated.

- LMA and LOW: the small $\Delta m^{2}$ part of LMA and the large $\Delta m^{2}$ part of LOW are reduced because they predict a day-night variation that is larger than observed. Both active LMA and active LOW solutions predict a flat spectrum in agreement with the observation.

\section{Global analysis}

In order to quantitatively discuss the combined analysis of the full bulk of solar neutrino data, one must define a global statistical function. How to implement such a program is a question that is being discussed intensively in the literature. ${ }^{1}$ The situation at present is that, although one finds slightly different globally allowed regions, depending on the particular prescription used in the combination, the main conclusions are independent of the details of the analysis.

\footnotetext{
${ }^{1}$ See, for example, Bahcall, Gonzalez-Garcia, and PeñaGaray, 2001, 2002a, 2002c; Creminelli, Segnorelli, and Strumia, 2001; Garzelli and Giunti, 2001; Barger, Marfatia, Whisnant, and Wood, 2002; de Holanda and Smirnov, 2002; Fogli, Lisi, Marrone, Montanino, and Palazzo, 2002.
} 


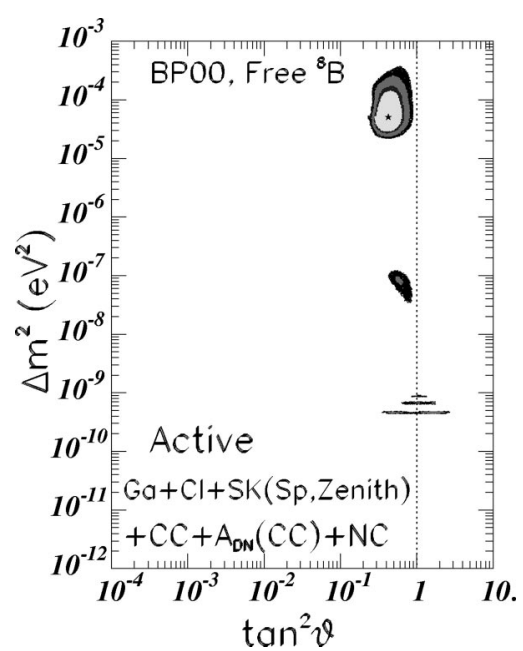

FIG. 18. Allowed oscillation parameters (at 90\%, 95\%, 99\%, and $99.7 \%$ C.L.) from the global analysis of the solar neutrino data. The best-fit point (large-mixing-angle active) is marked with a star.

We show in Fig. 18 and Table III the results from the global analysis of Bahcall, Gonzalez-Garcia, and PeñaGaray (2002c). In Fig. 18 we show the allowed regions which correspond to $90 \%, 95 \%, 99 \%$, and $99.73 \%(3 \sigma)$ C.L. for $\nu_{e}$ oscillations into active neutrinos. In Table III we list the local minima of the allowed regions, the value of $\chi_{\min }^{2}$ in each local minimum.

The results show that at present the most favored solution is the large-mixing-angle oscillation, while the low-mass solution provides the second best fit. There are some small allowed islands for vacuum oscillations. The active small-mixing-angle solution does not appear at $3 \sigma$ as a consequence of the incompatibility between the observed small charged-current rate at SNO, which would favor larger mixing, and the flat spectrum, which favors smaller mixing. Oscillations of solar neutrinos into a pure sterile state are also disfavored at the $5.4 \sigma$ level.

\section{ATMOSPHERIC NEUTRINOS}

Atmospheric neutrinos are produced in cascades initiated by collisions of cosmic rays with the Earth's atmosphere. Some of the mesons produced in these cascades, mostly pions and some kaons, decay into electron and muon neutrinos and antineutrinos. The predicted absolute fluxes of neutrinos produced by cosmic-ray interactions in the atmosphere are uncertain at the $20 \%$ level. The ratios of neutrinos of different flavor are, however, expected to be accurate to better than $5 \%$. Since $\nu_{e}$ is produced mainly from the decay chain $\pi \rightarrow \mu \nu_{\mu}$ followed by $\mu \rightarrow e \nu_{\mu} \nu_{e}$, one naively expects a 2:1 ratio of $\nu_{\mu}$ to $\nu_{e}$. (For higher-energy events, the expected ratio is smaller because some of the muons arrive at the Earth before they have had time to decay.) In practice, however, the theoretical calculation of the ratio of muonlike interactions to electronlike interactions in each experiment is more complicated.

Atmospheric neutrinos are observed in underground experiments using different techniques and leading to different type of events, which we briefly summarize here. They can be detected by the direct observation of their charged-current interaction inside the detector. These are the contained events. Contained events can be further classified into fully contained events, when the charged lepton (either electron or muon) that is produced in the neutrino interaction does not escape the detector, and partially contained muons, when the produced muon exits the detector. For fully contained events, the flavor, kinetic energy, and direction of the charged lepton can be best determined. As discussed later, some experiments further divide the contained data sample into sub- $\mathrm{GeV}$ and multi-GeV events, according to whether the visible energy is below or above $1.2 \mathrm{GeV}$. On average, sub-GeV events arise from neutrinos of several hundreds of $\mathrm{MeV}$, while multi-GeV events are originated by neutrinos with energies of the order of several GeV. Higher-energy muon neutrinos and antineutrinos can also be detected indirectly by observing the muons produced in their charged-current interactions in the vicinity of the detector. These are the socalled upgoing muons. Should the muon stop inside the detector, it is classified as a stopping muon (which arises from neutrinos $E_{\nu} \sim 10 \mathrm{GeV}$ ), while if the muon track crosses the full detector the event is classified as a through-going muon (which is originated by neutrinos with energies of the order of hundreds of GeV). Downgoing muons from $\nu_{\mu}$ interactions above the detector cannot be distinguished from the background of cosmic ray muons. Higher-energy $\nu_{e}$ 's cannot be detected this way as the produced $e$ showers immediately in the rock.

TABLE III. Best-fit global oscillation parameters with all solar neutrino data. This table corresponds to the global solution illustrated in Fig. 18. The last four solutions do not appear in Fig. 18 because their $\chi_{\min }^{2}$ is too large.

\begin{tabular}{lcccc}
\hline \hline Solution & $\Delta m^{2}\left[\mathrm{eV}^{2}\right]$ & $\tan ^{2} \theta$ & $f_{\mathrm{B}}$ & $\chi_{\min }^{2}$ (46 degrees of freedom) \\
\hline Large mixing angle & $5.0 \times 10^{-5}$ & $4.2 \times 10^{-1}$ & 1.07 & 45.5 \\
Low mass & $7.9 \times 10^{-8}$ & $6.1 \times 10^{-1}$ & 0.91 & 54.3 \\
Vacuum oscillations & $4.6 \times 10^{-10}$ & $1.8 \times 10^{0}$ & 0.77 & 52.0 \\
Small mixing angle & $5.0 \times 10^{-6}$ & $1.5 \times 10^{-3}$ & 0.89 & 62.7 \\
Just So & $5.8 \times 10^{-12}$ & $1.0 \times 10^{0}$ & 0.46 & 86.3 \\
Sterile vacuum oscillations & $4.6 \times 10^{-10}$ & $2.3 \times 10^{0}$ & 0.81 & 81.6 \\
Sterile small mixing angle & $3.7 \times 10^{-6}$ & $4.7 \times 10^{-4}$ & 0.55 & 89.3 \\
\hline \hline
\end{tabular}




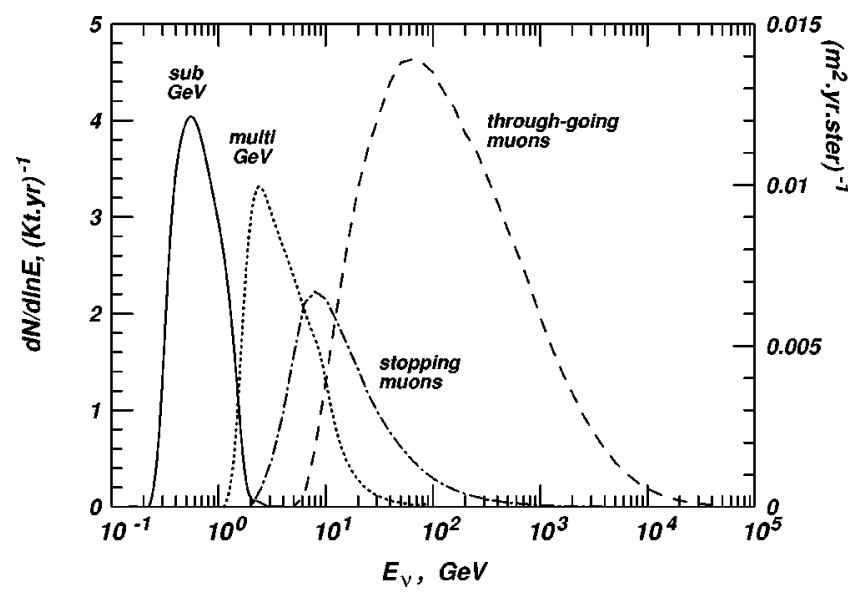

FIG. 19. Event rates as a function of neutrino energy for fully contained events, stopping muons, and through-going muons at SuperKamiokande. From Engel, Gaisser, and Stanev, 2000.

In Fig. 19 we display the characteristic neutrino energy distribution for these different type of events (taken from Engel, Gaisser, and Stanev, 2000).

Atmospheric neutrinos were first detected in the 1960s by the underground experiments in South Africa (Reines et al., 1965) and the Kolar Gold Field experiment in India (Achar et al., 1965). These experiments measured the flux of horizontal muons (they could not discriminate between downgoing and upgoing directions) and although the observed total rates were not in full agreement with theoretical predictions (Zatsepin and Kuzmin, 1962; Cowsik et al., 1965; Osborne, Said, and Wolfendale, 1965), the differences were not statistically significant.

Other experiments were proposed and built in the 1970s and 1980s. The original purpose was to search for nucleon decay, for which atmospheric neutrinos constitute background, although the possibility of using them to search for oscillation was also known (Ayres et al., 1984). Two different detection techniques were employed. In water Cerenkov detectors, the target is a large volume of water surrounded by photomultipliers which detect the Cerenkov ring produced by the charged leptons. The event is classified as an electronlike or muonlike event according to whether the ring is diffuse or sharp. In iron calorimeters, the detector is composed of a set of alternating layers of iron, which act as a target, and some tracking element (such as plastic drift tubes) which allows the reconstruction of the shower produced by the electrons or the tracks produced by muons. Both types of detectors allow for flavor classification of the events.

The two oldest iron calorimeter experiments, those of Fréjus (Daum et al., 1995) and NUSEX (Aglietta et al., 1989), found atmospheric neutrino fluxes in agreement with the theoretical predictions. On the other hand, two water Cerenkov detectors, IMB (Becker-Szendy et al., 1992) and Kamiokande, detected a ratio of $\nu_{\mu}$-induced events to $\nu_{e}$-induced events smaller than that expected by a factor of about 0.6. Kamiokande performed separate analyses for both sub-GeV neutrinos (Hirata et al.,

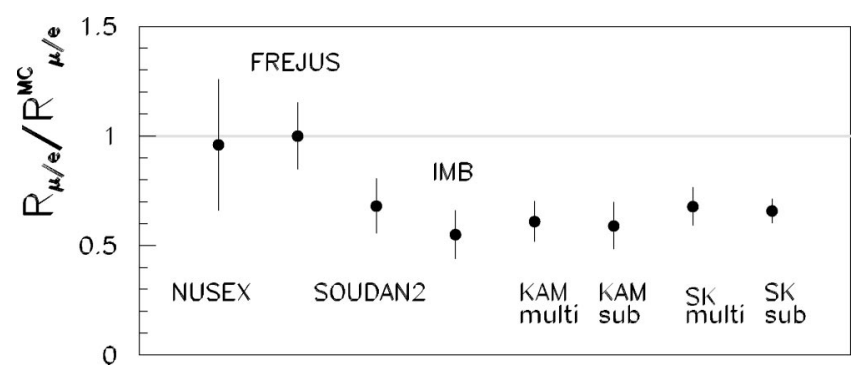

FIG. 20. The double ratio of $\nu_{\mu}$ to $\nu_{e}$ events, data divided by theoretical predictions, for various underground atmospheric neutrino detectors.

1992) and multi-GeV neutrinos (Fukuda et al., 1994), which showed the same deficit. This was the original formulation of the atmospheric neutrino anomaly. In Fig. 20 we show the values of the ratio $R_{\mu / e} / R_{\mu / e}^{M C}$, which denotes the double ratio of experimental-to-expected ratio of muonlike to electronlike events. Whether $R_{\mu / e} / R_{\mu / e}^{M C}$ was small because there was $\nu_{\mu}$ disappearance or $\nu_{e}$ appearance or a combination of both could not be determined. Furthermore, the fact that the anomaly appeared only in water Cerenkov and not in iron calorimeters left the window open for the suspicion of a possible systematic problem as the origin of the effect.

Kamiokande also presented the zenith angular dependence of the deficit for multi-GeV neutrinos (Fukuda et al., 1994). The zenith angle, parametrized in terms of $\cos \theta$, measures the direction of the reconstructed charged lepton with respect to the vertical of the detector. Vertically downgoing or upgoing particles correspond to $\cos \theta=+1$ or -1 . Horizontally arriving particles come at $\cos \theta=0$. Kamiokande results seemed to indicate that the deficit was mainly due to the neutrinos coming from below the horizon. Atmospheric neutrinos are produced isotropically at a distance of about $15 \mathrm{~km}$ above the surface of the Earth. Therefore neutrinos coming from the top of the detector have traveled approximately those $15 \mathrm{~km}$ before interacting, while those coming from the bottom of the detector have traversed the full diameter of the Earth, $\sim 10^{4} \mathrm{~km}$, before reaching the detector. The Kamiokande distribution suggested that the deficit increases with the distance between the neutrino production and interaction points.

In the last five years, the case for the atmospheric neutrino anomaly has become much stronger with the high precision and large statistics data from SK, and it has received important confirmation from the iron calorimeter detectors Soudan2 and MACRO.

SK is a 50-kiloton water Cerenkov detector constructed under Mt. Ikenoyama located in the central part of Japan, giving it a rock overburden of $2700 \mathrm{~m}$ water equivalent. The fiducial mass of the detector for atmospheric neutrino analysis is 22.5 kilotons. In June 1998, at the Neutrino98 conference, SK presented evidence of $\nu_{\mu}$ oscillations (Fukuda et al., 1998) based on the angular distribution for their contained event data sample. Since then SK has accumulated more statistics 

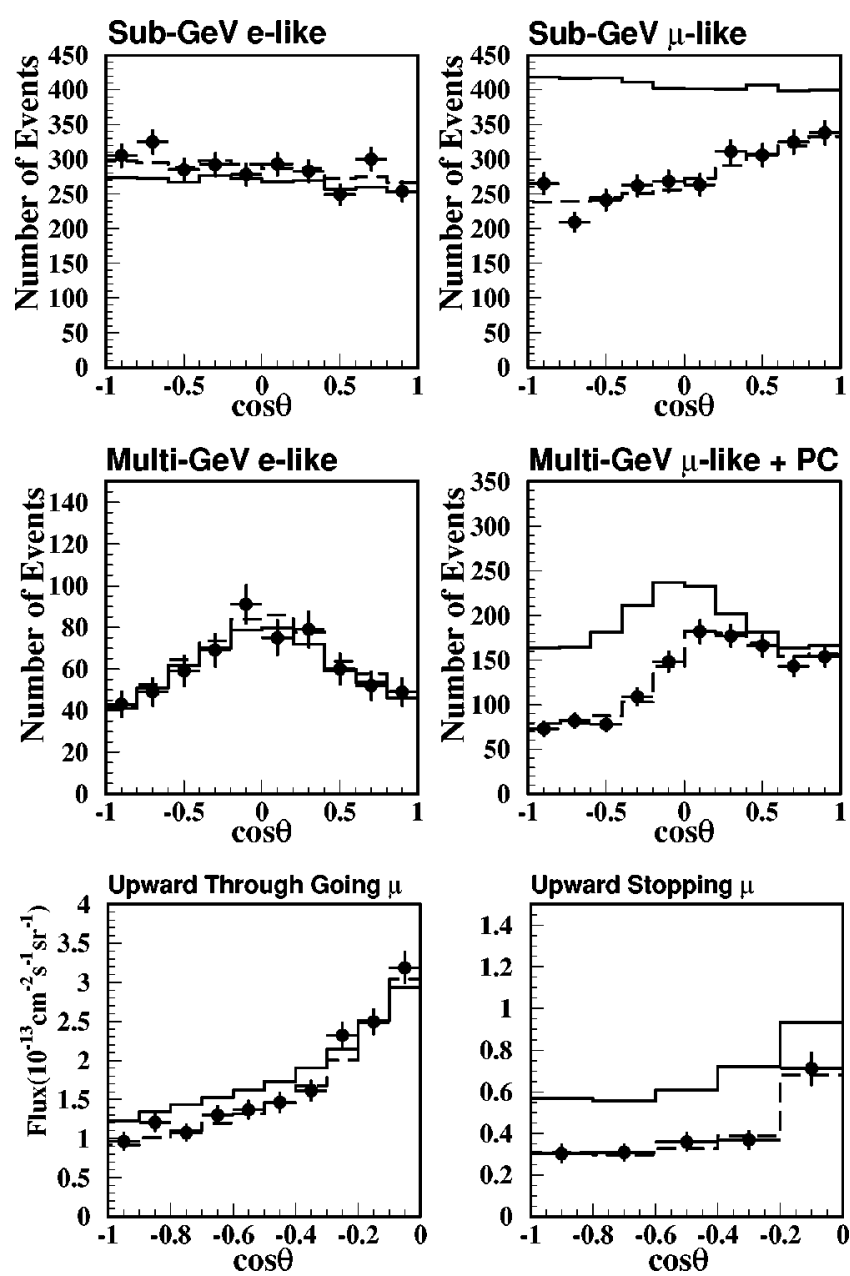

FIG. 21. Zenith-angle distribution of 1289 days' worth of SuperKamiokande data samples: dotted line, data; solid line, MC with no oscillation; dashed line, MC with best-fit oscillation parameters. From Toshito et al., 2001.

and has also studied the angular dependence of the upgoing muon sample (Fukuda et al., 1999a, 1999b). In Fig. 21 (from Toshito et al., 2001) we show their data as of summer 2001, corresponding to a 79-kiloton year (1289 days) exposure for their contained sub-GeV (2864 onering $e$-like events and 2788 one-ring $\mu$-like events) and multi-GeV (626 one-ring $e$-like events, 558 one-ring $\mu$-like events, and 754 events that were partially contained within the detector), as well as stopping muons and through-going muon samples (1416 events).

In the figure, we show the angular zenith distribution of the different samples. Comparing the observed and the expected Monte Carlo (MC) distributions, we can make the following statements:

$\nu_{e}$ distributions are well described by the $\mathrm{MC}$ while $\nu_{\mu}$ presents a deficit. Thus the atmospheric neutrino deficit is mainly due to disappearance of $\nu_{\mu}$ and not the appearance of $\nu_{e}$.

(ii) The suppression of contained $\mu$-like events is stronger for larger $\cos \theta$, which implies that the deficit grows with the distance traveled by the neutrino from its production point to the detector. This effect is more obvious for multi-GeV events because at higher energy the direction of the charged lepton is more aligned with the direction of the neutrino. It can also be described in terms of an up-down asymmetry:

$A_{\mu} \equiv \frac{U-D}{U+D}=-0.316 \pm 0.042$ (stat.) \pm 0.005 (syst.),

where $U(D)$ are the contained $\mu$-like events with zenith angle in the range $-1<\cos \theta<-0.2(0.2$ $<\cos \theta<1$ ). It deviates from the standard-model value, $A_{\mu}=0$, by 7.5 standard deviations.

(iii) The overall suppression of the flux of stopping muons, $\Phi_{\mathrm{ST}}$, is by a factor of about 0.6 , similar to that of contained events. However, for the flux of through-going muons, $\Phi_{\mathrm{TH}}$, the suppression is weaker, which implies that the effect is smaller at larger neutrino energy. This effect is also parametrized in terms of the double flux ratio:

$\frac{\Phi_{\mathrm{ST}} /\left.\Phi_{\mathrm{TH}}\right|_{\mathrm{obs}}}{\Phi_{\mathrm{ST}} /\left.\Phi_{\mathrm{TH}}\right|_{\mathrm{MC}}}=0.635 \pm 0.049$ (stat.) \pm 0.035 ( syst.)

$$
\pm 0.084 \text { (theo.) }
$$

which deviates from the standard-model value of 1 by about 3 standard deviations.

These effects have been confirmed by the results of the iron calorimeters Soudan2 and MACRO, which removed the suspicion that the atmospheric neutrino anomaly is simply a systematic effect in water detectors. In particular, Soudan2, which has mainly analyzed contained events (Allison et al., 1999), has measured a ratio $R_{\mu / e} / R_{\mu / e}^{\mathrm{MC}}=0.68 \pm 0.11 \pm 0.06$, in good agreement with the results from the water Cerenkov experiments. The main results from MACRO concern the angular distribution for through-going muons [see Ambrosio et al. (2001) for their latest data] and show good agreement with the results from SK. The Baksan experiment (Boliev et al., 1999) has also reported results on the angular distribution of through-going muons, but their data are less precise.

To analyze the atmospheric neutrino data in terms of oscillations, one needs to have a good understanding of the different elements entering into the theoretical predictions of the event rates: the atmospheric neutrino fluxes and their interaction cross section, which we describe next.

\section{A. Atmospheric neutrino fluxes}

Modern calculations of atmospheric neutrino fluxes consist of a Monte Carlo procedure that combines the measured energy spectra and chemical composition of the cosmic-ray flux at the top of the atmosphere with the properties of their hadronic interaction with light atmospheric nuclei followed by neutrino production from secondary $\pi, K$, and $\mu$ decay.

Present experiments use the neutrino flux calculations mainly from Honda et al. (1990, 1995, 1996), the Bartol group (Gaisser, Stanev, and Barr, 1988; Barr, Gaisser, and Stanev, 1989; Agrawal et al., 1996; Lipari, Gaisser, 
and Stanev, 1998) and Fiorentini, Naumov, and Villante (2001). These calculations have in common a onedimensional picture, in which all secondary particles in the showers, neutrinos included, are considered collinear with the primary cosmic rays (see also Bugaev and Naumov, 1989). The three-dimensional picture was first considered by Lee and Koh (1990) and most recently by Tserkovnyak et al. (2003) and Battistoni et al. (2000). [For detailed comparisons between the various simulations, see Gaisser et al. (1996), Lipari (2000), and Battistoni (2001).]

The flux of neutrinos of flavor $i$ coming from the direction $\Omega$ can be schematically written as $\phi_{\nu_{i}}(\Omega)$ $=\Sigma_{A} \Phi_{A} \otimes R_{A} \otimes Y_{p, n \rightarrow v_{i}}$. Here $\Phi_{A}$ is the primary cosmicray spectrum, $R_{A}$ is the geomagnetic cutoff for protons or light nuclei incident on the atmosphere from the direction $\Omega$, and $Y_{p, n \rightarrow \nu_{i}}$ is the yield per nucleon of $\nu_{i}$. The separation into different nuclear species of the cosmic-ray spectrum is necessary because the neutrino yield depends on the energy per nucleon of the incident cosmic rays, but the geomagnetic cutoff depends on the magnetic rigidity $(\mathcal{R}=p c / Z e)$ which, at the same energy per nucleon, depends on $A / Z$.

We now briefly summarize the uncertainties in each of these three ingredients of the calculation, namely, the primary cosmic-ray flux, the geomagnetic effects, and the hadronic interactions on light nuclei.

\section{Cosmic-ray spectrum}

The cosmic radiation incident at the top of the atmosphere includes all stable charged particles and nuclei. Apart from particles associated with solar flares, cosmic rays are assumed to come from outside the solar system. In Fig. 22 (from Simpson, 1983) we show a compilation of the major components of cosmic rays as a function of energy per nucleon. The following features of the cosmic-ray spectrum are relevant to atmospheric neutrino fluxes:

(i) Composition: Most of the primaries are protons, although the chemical composition varies with energy at low energies. Above a few $\mathrm{GeV}$ of energy per nucleon, about $79 \%$ of the primaries are protons, while helium, the next most abundant component, is about $3 \%$. This fraction remains constant till beyond $100 \mathrm{TeV}$.

(ii) Energy dependence: For energy above a few GeV, the cosmic-ray flux is a a steeply falling function of the energy: $d \phi / d E \propto E^{-3.7}$.

(iii) Solar modulation: At energies below $\sim 10 \mathrm{GeV}$, the incoming charged particles are modulated by the solar wind, which prevents the lower-energy cosmic rays of the inner solar system from reaching the Earth. Because of this effect there is an anticorrelation between the 11-year cycle of solar activity and the intensity of cosmic rays below 10 $\mathrm{GeV}$, which makes the cosmic-ray fluxes time dependent. The flux difference at solar maximum and solar minimum is more than a factor of 2 for

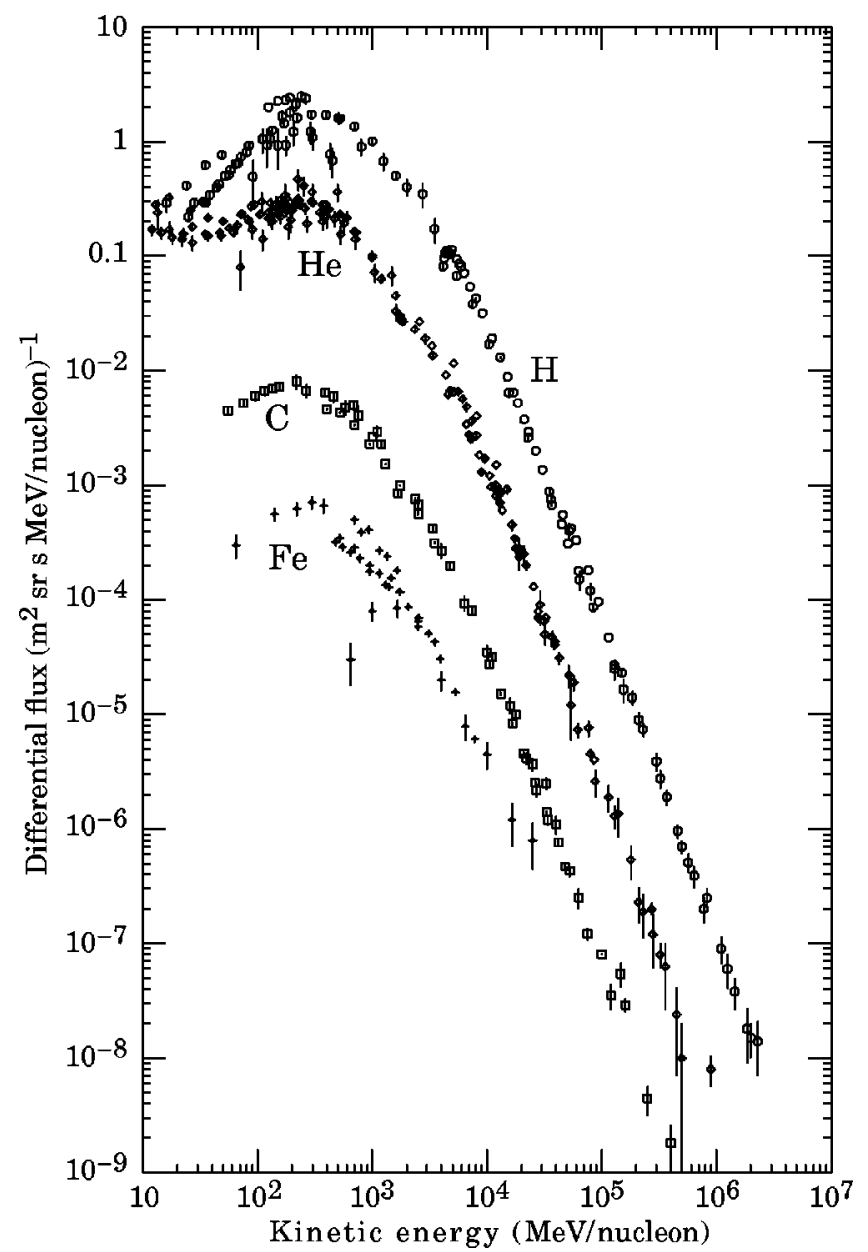

FIG. 22. The main components of the primary cosmic-ray spectrum. From Simpson, 1983.

$1-\mathrm{GeV}$ cosmic rays, and it decreases to $\sim 10 \%$ for $10-\mathrm{GeV}$ cosmic rays. The fluxes shown in Fig. 22 correspond to a particular epoch of the solar cycle.

\section{Geomagnetic effects}

Lower-energy cosmic rays are affected by the geomagnetic field that they must penetrate to reach the top of the atmosphere. This effect depends on the point where the cosmic rays arrive at the Earth (being stronger near the geomagnetic equator), and on their direction. Near the geomagnetic poles almost all primary particles can reach the atmosphere moving along field lines. In contrast, close to the geomagnetic equator the field restricts the flux at the top of the atmosphere to particles with energy larger than a few $\mathrm{GeV}$, the exact value depending on the direction of the particle trajectory. The relevant quantity is the magnetic rigidity, $\mathcal{R}=p c / Z e$, which parametrizes the characteristic radius of curvature of the particle trajectory in the presence of a magnetic field: particles with $\mathcal{R}$ below a local cutoff are bent away by the Earth's magnetic field and do not reach the atmosphere.

Given a map of the magnetic field around the Earth, we find that the value of the rigidity cutoff can be obtained from a computer simulation of cosmic-ray trajec- 
tories by using a backtracking technique, in which one determines whether a given primary particle's threemomentum and position correspond to an allowed or a forbidden trajectory. To do so, one integrates back the equation of motion of the cosmic-ray particle (actually one integrates the antiparticle equation) in the geomagnetic field and finds whether the past trajectory remains confined to a finite distance from the Earth-in which case it is a forbidden trajectory-or it originates from large enough $\left(\geq 10 R_{\oplus}\right)$ distances. The rigidity cutoff is calculated as the minimum $\mathcal{R}$ for which the trajectory is allowed or, in other words, the backtracked trajectory escapes from the magnetic field of the Earth.

The following features of the geomagnetic effects are relevant to the atmospheric neutrino fluxes:

(i) For a fixed direction, the cutoff rigidity grows monotonically from zero at the magnetic pole to a maximum value at the magnetic equator.

(ii) The cutoff rigidities for particles traveling toward the magnetic west are higher than those for particles traveling toward the magnetic east.

(iii) Thus the highest cutoff corresponds to westwardgoing, horizontal particles reaching the surface of the Earth at the magnetic equator.

\section{The neutrino yield}

As cosmic rays propagate in the atmosphere and interact with air nuclei, they create $\pi$ and $K$ mesons, which in turn decay and create atmospheric $\nu$ 's:

$$
\begin{aligned}
& A_{\mathrm{cr}}+A_{\mathrm{air}} \rightarrow \pi^{ \pm}, K^{ \pm}, K^{0}, \ldots ; \\
& \pi^{ \pm}, K^{ \pm} \rightarrow \mu^{ \pm}+\nu_{\mu}\left(\bar{\nu}_{\mu}\right), \\
& \mu^{ \pm} \rightarrow e^{ \pm}+\nu_{e}\left(\bar{\nu}_{e}\right)+\bar{\nu}_{\mu}\left(\nu_{\mu}\right) .
\end{aligned}
$$

We have displayed only the dominant channels. Since the decay distributions of the secondary mesons and muons are well known, the largest source of differences between the various calculations for the neutrino yield is the use of different models for the hadronic interactions (Gaisser et al., 1996). Since neutrinos produced by the decays of pions, kaons, and muons have different energy spectra, the main features of the interaction model which affect the $\nu_{\mu} / \nu_{e}$ composition and the energy and angular dependence of the neutrino fluxes are the $K / \pi$ ratio and their momentum distribution.

\section{The neutrino fluxes}

In Figs. 23 and 24 we show the atmospheric neutrino fluxes from the calculation of the Bartol group (Gaisser, Stanev, and Barr, 1988; Barr, Gaisser, and Stanev, 1989; Agrawal et al. 1996; Lipari, Gaisser, and Stanev, 1998) for the location of SK and for maximum solar activity. In the first figure we show the flux as a function of the neutrino energy averaged over the arrival direction. In the second figure we show the fluxes as a function of the zenith angle for various neutrino energies. We emphasize the following points:

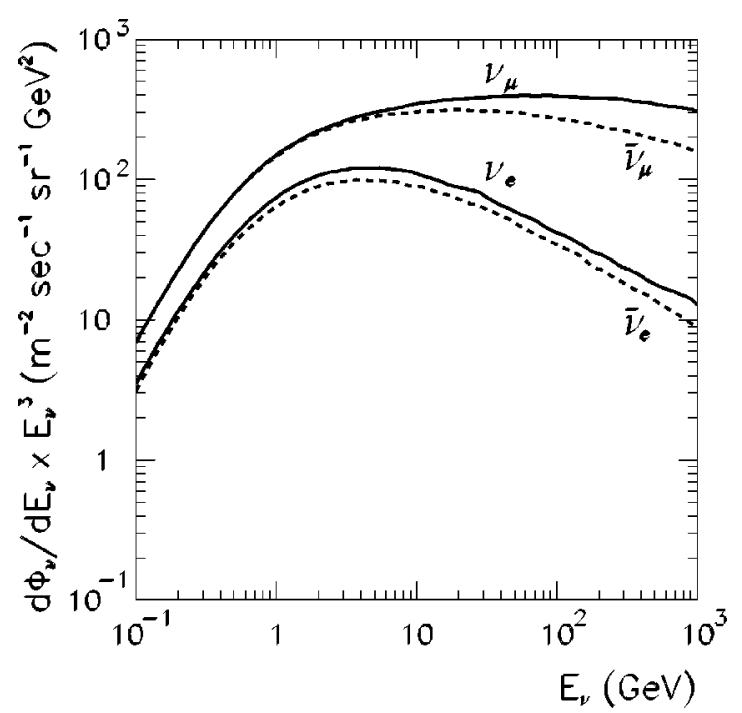

FIG. 23. Atmospheric neutrino fluxes as a function of neutrino energy.

(i) Energy dependence: For $E_{\nu} \gtrsim 1 \mathrm{GeV}$, the fluxes obey an approximate power law, $d \Phi / d E \propto E^{-\gamma}$ where $\gamma \sim 3$ (3.5) for muon (electron) neutrinos and antineutrinos. For $E_{\nu} \lesssim 1 \mathrm{GeV}$, the dependence on energy is weaker as a consequence of the bending of the cosmic-ray spectrum by geomagnetic effects and solar modulation.

(ii) $\bar{\nu} / \nu$ ratio: For $E \lesssim 1 \mathrm{GeV}$, when all pions and subsequent muons decay before reaching the Earth, the ratio $\bar{\nu}_{e} / \nu_{e}=\pi^{-} / \pi^{+}<1$. At these energies, the cosmic rays are mainly protons. The positively charged protons produce, on average, more $\pi^{+}$'s than $\pi^{-}$'s in their interactions. On the other hand, since $\pi^{+}\left(\mu^{+}\right)$produces a $\nu_{\mu}\left(\bar{\nu}_{\mu}\right)$ in its decay, we expect $\bar{\nu}_{\mu} / \nu_{\mu}=1$.

As energy increases, the secondary $\mu^{ \pm}$'s do not have time to decay before reaching the surface of the Earth and consequently $\bar{\nu}_{\mu} / \nu_{\mu}$ decreases.

(iii) $\left(\nu_{\mu}+\bar{\nu}_{\mu}\right) /\left(\nu_{e}+\bar{\nu}_{e}\right)$ ratio: At $E \lesssim 1 \mathrm{GeV}$, the ratio is very close to 2 , as expected from the chain decays in Eq. (108). At higher energies, the ratio decreases because, as mentioned above, the $\mu$ 's do

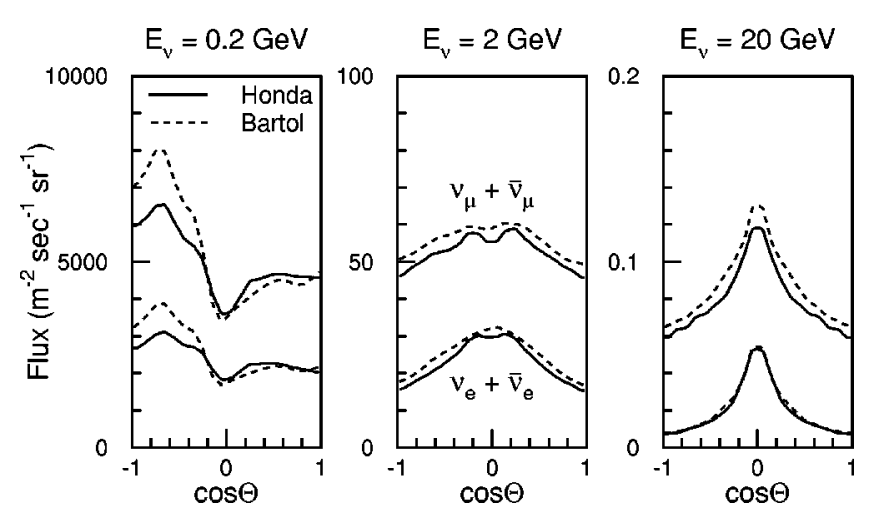

FIG. 24. Zenith-angle dependence of atmospheric neutrino fluxes. 


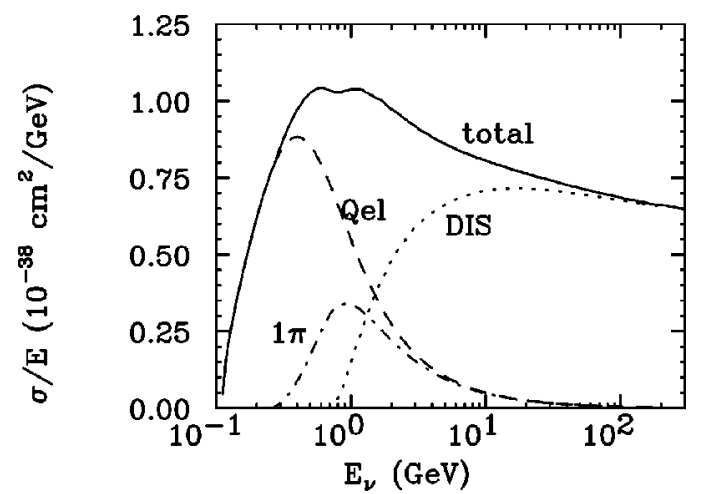

FIG. 25. The various contributions to the neutrino-nucleon cross section.

not have time to decay before reaching the Earth and fewer $\nu_{e}$ 's are produced.

(iv) Up-down asymmetry: At $E \lesssim \mathrm{a}$ few $\mathrm{GeV}$, the fluxes are up-down asymmetric due to geomagnetic effects. Geomagnetic effects are very small already at $E=2 \mathrm{GeV}$ (see Fig. 24) and the flux becomes up-down symmetric above that energy.

(v) Horizontal-vertical ratio: For $E \gtrsim$ a few $\mathrm{GeV}$, the fluxes are maximal for neutrinos arriving horizontally and minimal for neutrinos arriving vertically (see Fig. 24). This is due to the difference in the atmosphere density, which determines whether the pions decay before reinteracting with the air (thereby producing neutrinos). Pions arriving horizontally travel for a longer time in less dense atmosphere and are more likely to decay before reinteracting.

\section{B. Interaction cross sections}

In order to determine the expected event rates at the experiment, one needs to know the neutrino-nucleon interaction cross sections in the detector. The standard approach is to consider separately the contributions to this cross section from the exclusive channels of lower multiplicity (quasielastic scattering and single-pion production), and include all additional channels as part of the deep-inelastic scattering cross section:

$$
\sigma_{C C}=\sigma_{Q E}+\sigma_{1 \pi}+\sigma_{D I S} .
$$

In Fig. 25 we plot the cross sections for these processes.

The cross section for the quasielastic interaction is given by (Smith, 1972)

$$
\begin{aligned}
\frac{d \sigma_{Q E}}{d\left|q^{2}\right|}\left(\nu n \rightarrow \ell^{-} p\right)= & \frac{M^{2} G_{F}^{2} \cos ^{2} \theta_{c}}{8 \pi E_{\nu}^{2}} \\
& \times\left[A_{1}\left(q^{2}\right)-A_{2}\left(q^{2}\right) \frac{s-u}{M^{2}}\right. \\
& \left.+A_{3}\left(q^{2}\right) \frac{(s-u)^{2}}{M^{4}}\right],
\end{aligned}
$$

where $s-u=4 M E_{\nu}+q^{2}-m_{\ell}^{2}, M$ is the proton mass, $m_{\ell}$ is the charged-lepton mass, and $q^{2}$ is the momentum transfer. For $\bar{\nu} p \rightarrow \ell^{+} n$, a similar formula applies with the only change $A_{2} \rightarrow-A_{2}$. The functions $A_{1}, A_{2}$, and $A_{3}$ can be written in terms of axial and vector form factors:

$$
\begin{aligned}
A_{1}= & \frac{m_{\ell}^{2}-q^{2}}{4 M^{2}}\left[\left(4-\frac{q^{2}}{M^{2}}\right)\left|F_{A}\right|^{2}-\left(4+\frac{q^{2}}{M^{2}}\right)\left|F_{V}^{1}\right|^{2}\right. \\
& \left.-\frac{q^{2}}{M^{2}}\left|\xi F_{V}^{2}\right|^{2}-\frac{4 q^{2}}{M^{2}} \operatorname{Re}\left(F_{V}^{1 \star} \xi F_{V}^{2}\right)\right] \\
& -\frac{m_{\ell}^{2}}{M^{2}}\left(\left|F_{V}^{1}+\xi F_{V}^{2}\right|^{2}+\left|F_{A}\right|^{2}\right), \\
A_{2}= & -\frac{q^{2}}{M^{2}} \operatorname{Re}\left[F_{A}^{\star}\left(F_{V}^{1}+\xi F_{V}^{2}\right)\right], \\
A_{3}= & -\frac{1}{4}\left(\left|F_{A}\right|^{2}+\left|F_{V}^{1}\right|^{2}-\frac{q^{2}}{4 M^{2}}\left|\xi F_{V}^{2}\right|^{2}\right),
\end{aligned}
$$

where we neglected second-class currents and assumed conserved vector current. With this assumption all form factors are real and can be written as follows:

$$
\begin{aligned}
& F_{V}^{1}\left(q^{2}\right)=\left(1-\frac{q^{2}}{4 M^{2}}\right)^{-1}\left(1-\frac{q^{2}}{M_{V}^{2}}\right)^{-2} \\
& \times\left[1-\frac{q^{2}}{4 M^{2}}\left(1+\mu_{p}-\mu_{n}\right)\right], \\
& \xi F_{V}^{2}\left(q^{2}\right)=\left(1-\frac{q^{2}}{4 M^{2}}\right)^{-1}\left(1-\frac{q^{2}}{M_{V}^{2}}\right)^{-2}\left(\mu_{n}-\mu_{p}\right), \\
& F_{A}=F_{A}(0)\left(1-\frac{q^{2}}{4 M_{A}^{2}}\right)^{-2} .
\end{aligned}
$$

Here $\mu_{p}$ and $\mu_{n}$ are the proton and neutron anomalous magnetic moments and $M_{V}^{2}=0.71 \mathrm{GeV}^{2}$ is the vector mass, which is measured with high precision in electronscattering experiments. The largest uncertainties in this calculation are associated with the axial form factor. For example, the axial mass used by various collaborations varies in the range $M_{A}^{2}=0.71-1.06 \mathrm{GeV}^{2}$.

So far we have neglected nuclear effects. The most important of these effects is related to the Pauli principle and can be included by using a simple Fermi-gas model. In this approximation, the cross section of a bound nucleon is equal to the cross section of a free nucleon multiplied by a $q^{2}$-dependent factor which can be found, for instance, in Smith (1972). The effect of this factor is to decrease the cross section. The decrease is larger for smaller neutrino energy. For energies above 1 $\mathrm{GeV}$, the nuclear effects lead to an $8 \%$ decrease in the quasielastic cross section. As can be seen in Fig. 25, quasielastic interactions dominate for $E_{\nu} \lesssim 1 \mathrm{GeV}$ and induce most of the observed contained events.

In the interpretation of the zenith-angle distribution of contained events, an important role is played by the relative angle between the direction of the incoming 
neutrino (carrying the information on the neutrino path length) and the direction of the produced charged lepton (which is measured). For energies below $1 \mathrm{GeV}$, the opening angle is rather large: for sub-GeV events the correlation between the measured $\ell$ direction and the distance traveled by the neutrinos is weak. For $E \geq a$ few $\mathrm{GeV}$, the two directions are almost aligned (for example, it is on average $1^{\circ}$ for upgoing muons), and the $\ell$ direction gives a very good measurement of the neutrino path length.

Single-pion production, $\nu N \rightarrow \ell^{-} N^{\prime} \pi$, is dominated by the $\Delta(1232)$ resonance (Fogli and Nardulli, 1979; Nakahata et al., 1986). It is most relevant at $E_{\nu} \simeq 1 \mathrm{GeV}$ (see Fig. 25).

Deep-inelastic processes, $\nu N \rightarrow \ell^{-} X$ where $X$ represents any hadronic system, dominate atmospheric neutrino interactions for $E_{\nu} \gtrsim \mathrm{a}$ few $\mathrm{GeV}$. The parton model cross section is given by

$\frac{d \sigma_{D I S}(\nu)}{d x d y}=\frac{G_{F}^{2} s x}{4 \pi}\left[F_{1}-F_{3}+\left(F_{1}+F_{3}\right)(1-y)^{2}\right]$,

where $y=1-E_{\ell} / E_{\nu}$ and $x=-q^{2} /\left(2 M E_{\nu} y\right)$. For $\bar{\nu}$, a similar formula applies, with $F_{3} \rightarrow-F_{3} . F_{1}$ and $F_{3}$ are given in terms of the parton distributions. For isoscalar targets $F_{1}=2 \Sigma_{i}\left(q_{i}+\bar{q}_{i}\right)$ and $F_{3}=\Sigma_{i}\left(\bar{q}_{i}-q_{i}\right)$. In order to avoid double counting of the single-pion production, the deep-inelastic contribution must be integrated in the region of hadronic masses $W_{X}>W_{c} \quad\left(W_{c}=1.4 \mathrm{GeV}\right)$, which implies $2 M E_{\nu} y(1-x) \geqslant W_{c}^{2}-M^{2}$.

\section{Two-neutrino oscillation analysis}

The simplest and most direct interpretation of the atmospheric neutrino anomaly is that of muon neutrino oscillations (Barger and Whisnant, 1988; Hidaka, Honda, and Midorikawa, 1988; Learned, Pakvasa, and Weiler, 1988). The estimated value of the oscillation parameters can be easily derived in the following way:

- The angular distribution of contained events shows that, for $E \sim 1 \mathrm{GeV}$, the deficit comes mainly from $L$ $\sim 10^{2}-10^{4} \mathrm{~km}$. The corresponding oscillation phase must be maximal, $\Delta m^{2}\left(\mathrm{eV}^{2}\right) L(\mathrm{~km}) / 2 E(\mathrm{GeV}) \sim 1$, which requires $\Delta m^{2} \sim 10^{-4}-10^{-2} \mathrm{eV}^{2}$.

- Assuming that all upgoing $\nu_{\mu}$ 's which would lead to multi-GeV events oscillate into a different flavor while none of the downgoing ones do, the up-down asymmetry is given by $\left|A_{\mu}\right|=\sin ^{2} 2 \theta /\left(4-\sin ^{2} 2 \theta\right)$. The present one-sigma bound reads $\left|A_{\mu}\right|>0.27$ [see Eq. (106)], which requires that the mixing angle be close to maximal, $\sin ^{2} 2 \theta>0.85$.

In order to go beyond these rough estimates, one must compare in a statistically meaningful way the experimental data with the detailed theoretical expectations. We now describe how to obtain the allowed region. We consider two neutrino cases, where $\nu_{\mu}$ oscillates into either $\nu_{e}$ or $\nu_{\tau}$ or a sterile neutrino $\nu_{s}$.

\section{Predicted number of events}

For a given neutrino oscillation channel, the expected number of $\mu$-like and $e$-like contained events, $N_{\alpha}(\alpha$ $=\mu, e)$, can be computed as

$$
N_{\mu}=N_{\mu \mu}+N_{e \mu}, \quad N_{e}=N_{e e}+N_{\mu e},
$$

where

$$
\begin{aligned}
N_{\alpha \beta}= & n_{t} T \int \frac{d^{2} \Phi_{\alpha}}{d E_{\nu} d\left(\cos \theta_{\nu}\right)} \kappa_{\alpha}\left(h, \cos \theta_{\nu}, E_{\nu}\right) \\
& \times P_{\alpha \beta} \frac{d \sigma}{d E_{\beta}} \varepsilon\left(E_{\beta}\right) d E_{\nu} d E_{\beta} d\left(\cos \theta_{\nu}\right) d h .
\end{aligned}
$$

Here $P_{\alpha \beta}$ is the conversion probability of $\nu_{\alpha} \rightarrow \nu_{\beta}$ for given values of $E_{\nu}, \cos \theta_{\nu}$ and $h$, that is, $P_{\alpha \beta} \equiv P\left(\nu_{\alpha}\right.$ $\left.\rightarrow \nu_{\beta} ; E_{\nu}, \cos \theta_{\nu}, h\right)$. In the standard model, the only nonzero elements are the diagonal ones, i.e., $P_{\alpha \alpha}=1$ for all $\alpha$. In Eq. (115), $n_{t}$ denotes the number of target particles, $T$ is the experiment running time, $E_{\nu}$ is the neutrino energy, $\Phi_{\alpha}$ is the flux of atmospheric $\nu_{\alpha}$ 's, $E_{\beta}$ is the final charged-lepton energy, $\varepsilon\left(E_{\beta}\right)$ is the detection efficiency for such a charged lepton, $\sigma$ is the neutrinonucleon interaction cross section, and $\theta_{\nu}$ is the angle between the vertical direction and the incoming neutrinos ( $\cos \theta_{\nu}=1$ corresponds to the down-coming neutrinos). In Eq. (115), $h$ is the slant distance from the production point to sea level for $\alpha$-type neutrinos with energy $E_{\nu}$ and zenith angle $\theta_{\nu}$, and $\kappa_{\alpha}$ is the slant distance distribution, normalized to one. (We use in our calculations $\kappa_{\alpha}$ from Gaisser and Stanev, 1998.)

To obtain the expectation for the angular distribution of contained events, one must integrate the corresponding bins for $\cos \theta_{\beta}$, where $\theta_{\beta}$ is the angle of the detected lepton, taking into account the opening angle between the neutrino and the charged-lepton directions as determined by the kinematics of the neutrino interaction.

As discussed above, the neutrino fluxes, in particular those in the sub-GeV range, depend on the solar activity. In order to take this fact into account, one uses in Eq. (115) a linear combination of atmospheric neutrino fluxes $\Phi_{\alpha}^{\max }$ and $\Phi_{\alpha}^{\min }$ which correspond to the most (solar maximum) and least (solar minimum) active phases of the Sun, respectively, with different weights which depend on the specific running period.

Experimental results on upgoing muons are presented in the form of measured muon fluxes. To obtain the effective muon fluxes for both stopping and through-going muons, one must convolute the survival probabilities for $\nu_{\mu}$ 's with the corresponding muon fluxes produced by neutrino interactions with the Earth. One must further take into account the muon energy loss during propagation both in the rock and in the detector, and also the effective detector area for both types of events, stopping and through-going. Schematically,

$$
\begin{aligned}
\Phi_{\mu}(\theta)_{S, T}= & \frac{1}{A\left(L_{\min }, \theta\right)} \int_{E_{\mu, \min }}^{\infty} \frac{d \Phi_{\mu}\left(E_{\mu}, \cos \theta\right)}{d E_{\mu} d \cos \theta} \\
& \times A_{S, T}\left(E_{\mu}, \theta\right) d E_{\mu},
\end{aligned}
$$


where

$$
\begin{aligned}
\frac{d \Phi_{\mu}}{d E_{\mu} d \cos \theta}= & N_{A} \int_{E_{\mu}}^{\infty} d E_{\mu 0} \int_{E_{\mu 0}}^{\infty} d E_{\nu} \int_{0}^{\infty} d X \int_{0}^{\infty} d h \\
& \times \kappa_{\nu_{\mu}}\left(h, \cos \theta, E_{\nu}\right) \frac{d \Phi_{\nu_{\mu}}\left(E_{\nu}, \theta\right)}{d E_{\nu} d \cos \theta} \\
& \times P_{\mu \mu} \frac{d \sigma\left(E_{\nu}, E_{\mu 0}\right)}{d E_{\mu 0}} F_{\text {rock }}\left(E_{\mu 0}, E_{\mu}, X\right) .
\end{aligned}
$$

Here $N_{A}$ is the Avogadro number, $E_{\mu 0}$ is the energy of the muon produced in the neutrino interaction, $E_{\mu}$ is the muon energy when entering the detector after traveling a distance $X$ in the rock, and $\cos \theta$ labels both the neutrino and the muon directions which, at the relevant energies, are collinear to a very good approximation. We denote by $F_{\text {rock }}\left(E_{\mu 0}, E_{\mu}, X\right)$ the function that characterizes the energy spectrum of the muons arriving at the detector. The standard practice is to use an analytical approximation obtained by neglecting fluctuations during muon propagation in the Earth. In this case the three quantities $E_{\mu 0}, E_{\mu}$, and $X$ are not independent:

$$
\int_{0}^{\infty} F_{\text {rock }}\left(E_{\mu 0}, E_{\mu}, X\right) d X=\frac{1}{\left\langle d \mathcal{E}_{\mu}\left(E_{\mu}\right) / d X\right\rangle},
$$

where $\left\langle d \mathcal{E}_{\mu}\left(E_{\mu}\right) / d X\right\rangle$ is the average muon energy loss due to ionization, bremsstrahlung, $e^{+} e^{-}$pair production, and nuclear interactions in the rock (Lohmann, Kopp, and Voss, 1985).

For SK, the pathlength traveled by the muon inside the detector is given by the muon range function in water:

$$
L\left(E_{\mu}\right)=\int_{0}^{E_{\mu}} \frac{1}{\left\langle d \mathcal{E}_{\mu}\left(E_{\mu}^{\prime}\right) / d X\right\rangle} d E_{\mu}^{\prime} .
$$

In Eq. (116), $A\left(L_{\min }, \theta\right)=A_{S}\left(E_{\mu}, \theta\right)+A_{T}\left(E_{\mu}, \theta\right)$ is the projected detector area for internal path lengths longer than $L_{\text {min }}$ ( $=7 \mathrm{~m}$ in SK). Here $A_{S}$ and $A_{T}$ are the effective areas for stopping and through-going muon trajectories. These effective areas can be computed using a simple geometrical picture given, for instance, by Lipari and Lusignoli (1998). For a given angle, the threshold energy cut for SK muons is obtained by equating Eq. (119) to $L_{\min }$, i.e., $L\left(E_{\mu}^{\text {th }}\right)=L_{\min }$.

In contrast to $\mathrm{SK}$, MACRO present their results as muon fluxes for $E_{\mu}>1 \mathrm{GeV}$, after correcting for detector acceptances. Therefore in this case we compute the expected fluxes as in Eqs. (116) and (117) but without the inclusion of the effective areas.

\section{Conversion probabilities}

We consider a two-flavor scenario, $\nu_{\mu} \rightarrow \nu_{X}(X=e, \tau$, or $s$ ). The oscillation probabilities are obtained by solving the evolution equation of the $\nu_{\mu}-\nu_{X}$ system in the matter background of the Earth (see Sec. III.C):

$$
i \frac{d}{d t}\left(\begin{array}{c}
\nu_{\mu} \\
\nu_{X}
\end{array}\right)=\left(\begin{array}{cc}
H_{\mu} & H_{\mu X} \\
H_{\mu X} & H_{X}
\end{array}\right)\left(\begin{array}{c}
\nu_{\mu} \\
\nu_{X}
\end{array}\right),
$$

where

$$
\begin{aligned}
& H_{\mu}=V_{\mu}-\frac{\Delta m^{2}}{4 E_{\nu}} \cos 2 \theta, \\
& H_{X}=V_{X}+\frac{\Delta m^{2}}{4 E_{\nu}} \cos 2 \theta, \\
& H_{\mu X}=\frac{\Delta m^{2}}{4 E_{\nu}} \sin 2 \theta .
\end{aligned}
$$

The various neutrino potentials in matter are given by $V_{e}=V_{C}+V_{N}, \quad V_{\mu}=V_{\tau}=V_{N}$, and $V_{s}=0$ where the charged-current and neutral-current potentials $V_{C}$ and $V_{N}$ are proportional to the electron and neutron number density (see Sec. III.B), or equivalently, to the the matter density in the Earth (Dziewonski and Anderson, 1981). For antineutrinos, the signs of the potentials are reversed.

For $X=\tau$, we have $V_{\mu}=V_{\tau}$ and consequently these potentials can be removed from the evolution equation. The solution of Eq. (120) is then straightforward and the probability takes the well-known vacuum form [Eq. (34)], which is equal for neutrinos and antineutrinos. For $X=e$ or $s$, the effect of the matter potentials requires a numerical solution of the evolution equations in order to obtain $P_{\alpha \beta}$ which, furthermore, is different for neutrinos and antineutrinos. As a first approximation, one can use a constant Earth matter density. Then (see Sec. III) the solutions take the same form as the vacuum probability [Eq. (34)], but with the mixing angle and the oscillation length replaced by their effective values in matter [Eqs. (55) and (58) with $\left.A=2 E\left(V_{\mu}-V_{X}\right)\right]$. In Fig. 26 (from Lipari and Lusignoli, 1998) we show the survival probability of $\nu_{\mu}$ for the different oscillation channels for $\Delta m^{2}=5 \times 10^{-3} \mathrm{eV}^{2}, \sin ^{2} 2 \theta=1$, and various values of $E_{\nu}$. As can be seen in the figure, matter effects damp the oscillation amplitude. For the chosen mass difference, they are important for neutrino energies of few tens of $\mathrm{GeV}$ and therefore are relevant mainly for upgoing muons. We return to this point below when describing the results of the analysis.

\section{Statistical analysis}

In order to define in a statistically meaningful way the regions of neutrino flavor parameters that are allowed by a given set of atmospheric neutrino observables, one can construct, for example, a $\chi^{2}$ function, in a similar fashion to that described in Sec. IV.D.2 for the case of solar neutrino rate analysis. Details on the statistical analysis of the atmospheric neutrino data and results from the analyses with various data samples can be found in the literature. ${ }^{2}$

\footnotetext{
${ }^{2}$ See, for example, Fogli, Lisi, and Montanino (1994, 1995); Fogli and Lisi (1995b); Foot, Volkas, and Yasuda (1998); Gonzalez-Garcia, Nunokwa, et al. (1998); Yasuda (1998); Akhmedov et al. (1999); Gonzalez-Garcia, Nunokwa, et al. (1999); Gonzalez-Garcia, Fornengo, and Valle (2000).
} 


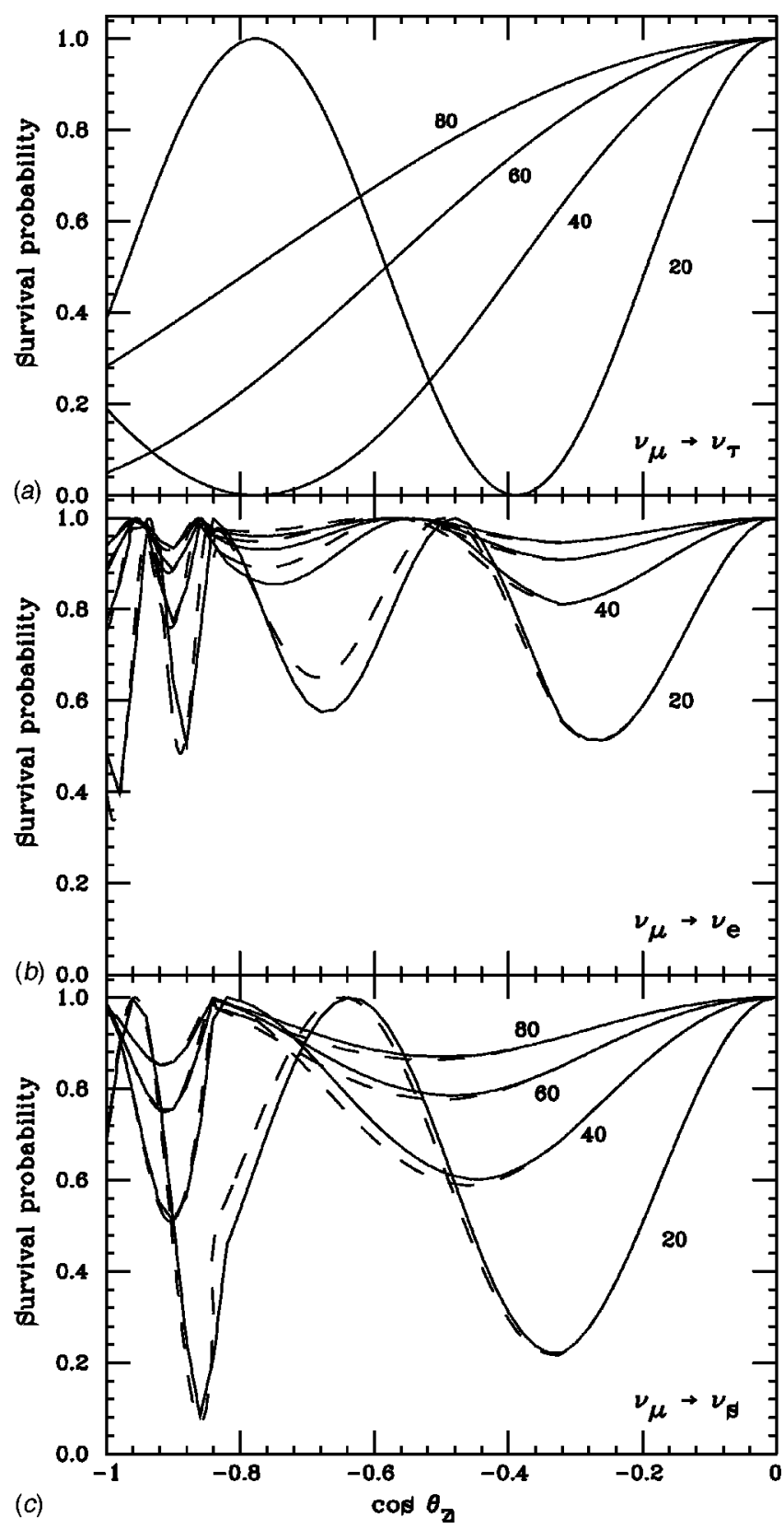

FIG. 26. $P_{\mu \mu}$ as a function of the zenith angle for maximal mixing of $\nu_{\mu}$ (a) with $\nu_{\tau}$, (b) $\nu_{e}$, and (c) $\nu_{s}$. For $\Delta m^{2}=5$ $\times 10^{-3} \mathrm{eV}^{2}$ the curves correspond to $E_{\nu}$ of $20,40,60$, and 80 $\mathrm{GeV}$. The dashed curves are calculated with the approximation of constant average densities in the mantle and in the core of the Earth. From Lipari and Lusignoli, 1998.

The general strategy is to compute the $\chi^{2}$ as a function of the neutrino parameters. By minimizing $\chi^{2}$ with respect to $\sin ^{2} 2 \theta$ and $\Delta m^{2}$, one determines the best-fit results, while the allowed regions are determined by the conditions: $\chi^{2} \equiv \chi_{\min }^{2}+4.61,6.1$, or 9.21 for a confidence level (C.L.) of $90 \%, 95 \%$, or $99 \%$, respectively.

\section{4. $\nu_{\mu} \rightarrow \nu_{e}$}

At present $\nu_{\mu} \rightarrow \nu_{e}$ is excluded with high C.L. as the explanation of the atmospheric neutrino anomaly for two different reasons:
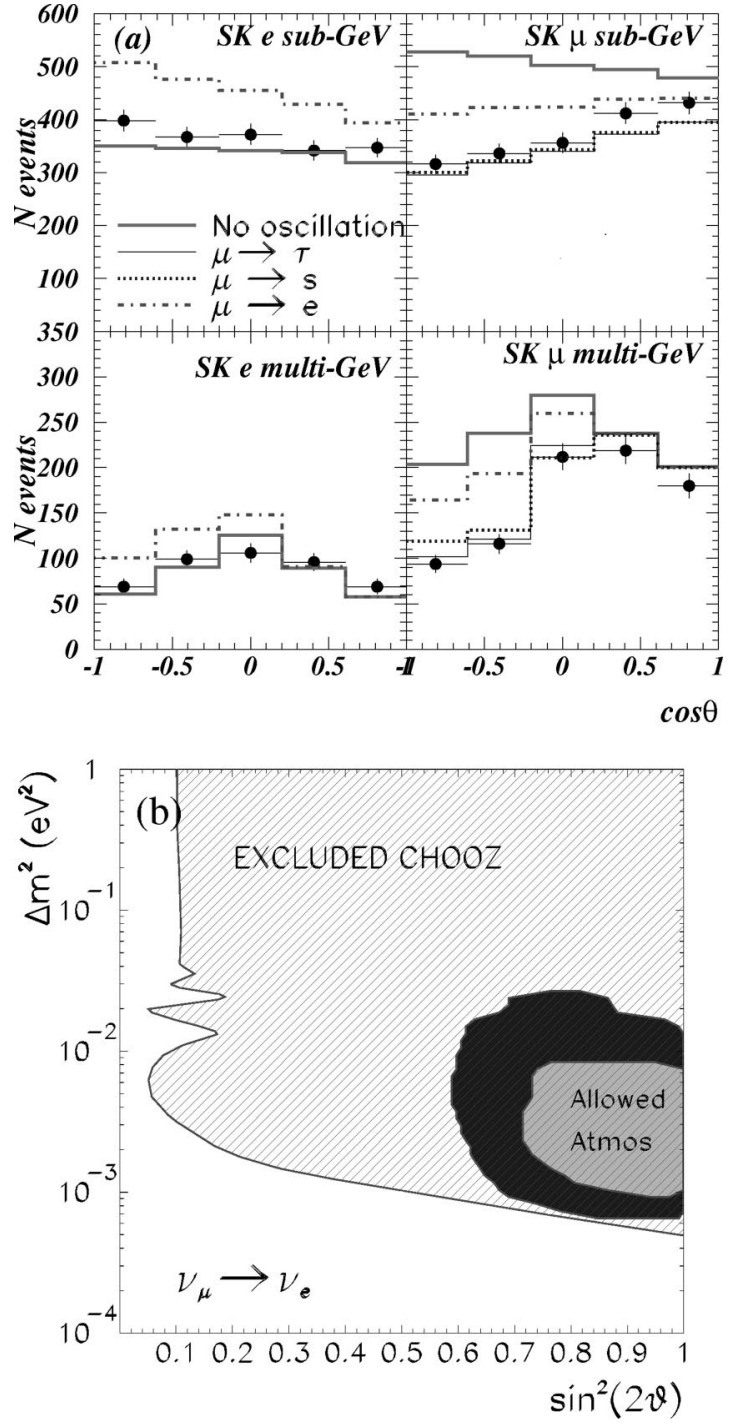

FIG. 27. The status of the $\nu_{\mu} \rightarrow \nu_{e}$ oscillation solution to the atmospheric neutrino anomaly.

(i) SK high-precision data show that the $\nu_{e}$ contained events are very well described by the standard-model prediction both in normalization and in their zenithangle dependence (see Fig. 21). The $\nu_{\mu}$ distribution, however, shows an angle-dependent deficit. $\nu_{\mu} \rightarrow \nu_{e}$ oscillations could explain the angular dependence of the $\nu_{\mu}$ flux only at the price of introducing angular dependence of the $\nu_{e}$ flux, in contrast to the data. Furthermore, even the best-fit point for $\nu_{\mu} \rightarrow \nu_{e}$ oscillations does not generate the observed up-down asymmetry in the multi-GeV muon sample. This is illustrated in Fig. 27, where we show the predicted angular distribution of contained events at SK for the best-fit points of the different oscillation channels. For $\nu_{\mu} \rightarrow \nu_{e}$ oscillations, the asymmetry in the multi-GeV muon distribution is much smaller than in the $\nu_{\mu} \rightarrow \nu_{\tau}$ or $\nu_{\mu} \rightarrow \nu_{s}$ channels.

(ii) Explaining the atmospheric data with $\nu_{\mu} \rightarrow \nu_{e}$ transition has direct implications for the $\bar{\nu}_{e} \rightarrow \bar{\nu}_{\mu}$ transition. In particular, there should be a $\bar{\nu}_{e}$ deficit in the CHOOZ reactor experiment. Thus the neutrino parameters not only give a poor fit to the atmospheric data but are ac- 


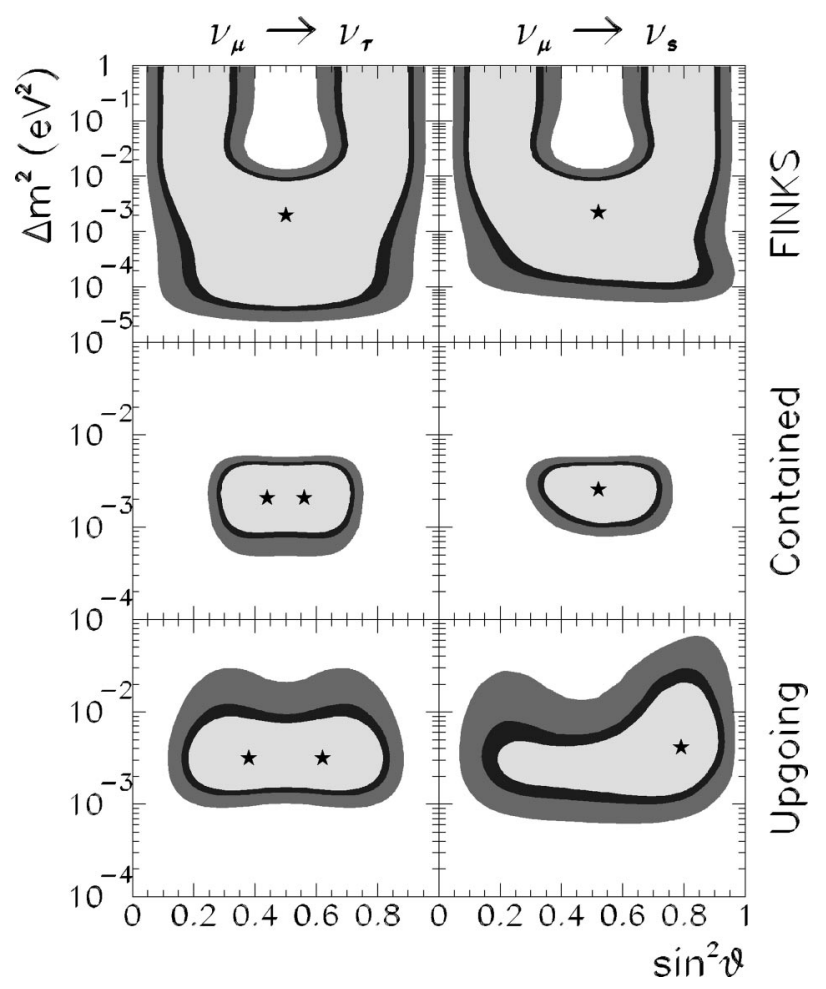

FIG. 28. Allowed regions (at 90\%, 95\%, and 99\% C.L.) from the analysis of various partial data samples of atmospheric neutrinos for the oscillation channels $\nu_{\mu} \rightarrow \nu_{\tau}$ and $\nu_{\mu} \rightarrow \nu_{s}$. The best-fit points are marked with a star (see text for details).

tually excluded by the negative results from the CHOOZ reactor experiment (see Fig. 27).

\section{5. $\nu_{\mu} \rightarrow \nu_{\tau}$ and $\nu_{\mu} \rightarrow \nu_{S}$}

In Fig. 28 we show the values of the oscillation parameters $\left(\Delta m^{2}, \sin ^{2} \theta\right)$ which describe various sets of data for these two oscillation channels. The upper panels (labeled as FINKS) and the central panels refer to contained events. The upper panels take into account only the total rates, and the central ones only the angular distribution (from both Kamiokande and SK). The lower panels correspond to the angular distribution for upgoing muons from SK. The three shaded regions are allowed at the 90\%, 95\%, and 99\% C.L. In Fig. 29 we plot the allowed regions from the global analysis, including all the atmospheric neutrino data. Also shown are the expected sensitivities from $\nu_{\mu}$ disappearance at the long-baseline experiments $\mathrm{K} 2 \mathrm{~K}$ and MINOS discussed in Sec. VI.D. (These figures are an update of the results presented by Gonzalez-Garcia, Fornengo, and Valle, 2000.) We emphasize the following points:

(i) The allowed regions from the various data samples overlap. The oscillation hypothesis can then consistently explain the atmospheric neutrino data.

(ii) The information from the total event rates alone is consistent with arbitrarily high $\Delta m^{2}$ values. The

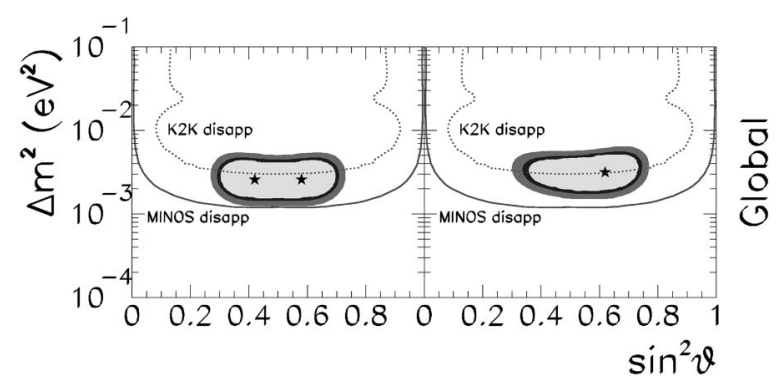

FIG. 29. Allowed regions (at 90\%, 95\%, and 99\% C.L.) from the analysis of the full data sample of atmospheric neutrinos for the oscillation channels $\nu_{\mu} \rightarrow \nu_{\tau}$ and $\nu_{\mu} \rightarrow \nu_{s}$. The best-fit points are marked with a star (see text for details). Also shown are the expected sensitivities from long-baseline experiments.

reason is that no information on the minimum oscillation length can be inferred from the data (see Sec. III.A).

(iii) The allowed regions for $\nu_{\mu} \rightarrow \nu_{\tau}$ transition are symmetric with respect to maximal mixing. This must be the case because the corresponding probabilities take the vacuum expression and therefore depend on $\sin ^{2} 2 \theta$.

(iv) The allowed regions for $\nu_{\mu} \rightarrow \nu_{s}$ transition are asymmetric due to Earth matter effects. These effects are more pronounced when the condition for maximal matter effect, $\Delta m^{2} \cos 2 \theta \sim 2 E V_{s}$, is fulfilled. Since in the chosen convention the potential difference $A=2 E\left(V_{\mu}-V_{s}\right)<0$, matter effects enhance neutrino oscillations for $\cos 2 \theta<0\left(\sin ^{2} \theta\right.$ $>0.5)$. The opposite situation holds for antineutrinos, but neutrino fluxes are larger and dominate in the resulting effect. As a result the allowed regions are wider on the $\sin ^{2} \theta>0.5$ side of the plot.

(v) The best fit to the full data for $\nu_{\mu} \rightarrow \nu_{\tau}$ corresponds to $\Delta m^{2}=2.6 \times 10^{-3} \mathrm{eV}^{2}$ and $\sin ^{2} 2 \theta=0.97$. The best fit for $\nu_{\mu} \rightarrow \nu_{s}$ lies at $\Delta m^{2}=3$ $\times 10^{-3} \mathrm{eV}^{2}$ and $\sin ^{2} \theta=0.61$.

In order to discriminate between the $\nu_{\mu} \rightarrow \nu_{\tau}$ and $\nu_{\mu}$ $\rightarrow \nu_{s}$ options, one can use the difference in the survival probabilities due to the presence of matter effects for oscillations into sterile neutrinos (Lipari and Lusignoli, 1998). As discussed above, the effect is important mainly for the higher-energy neutrinos which lead to throughgoing muons events. In Fig. 30 we plot the expected distributions for the best-fit points for the two channels. As can be seen in the figure, the distribution is steeper for $\nu_{\mu} \rightarrow \nu_{\tau}$, while for $\nu_{\mu} \rightarrow \nu_{s}$ a flattening is observed for neutrinos coming close to the vertical due to the damping of the oscillation amplitude (see Fig. 26). The data favor the steeper distributions and this translates into a better global fit for oscillations into $\nu_{\tau}$. For the global analysis, an update of the results of Gonzalez-Garcia, Fornengo, and Valle (2000) shows that for $\nu_{\tau}$ oscillations $\chi_{\min }^{2}=56 / 63$ degrees of freedom, while for $\nu_{s}$ oscillations $\chi_{\min }^{2}=72 / 63$ degrees of freedom.

SK has also used other methods to distinguish be- 


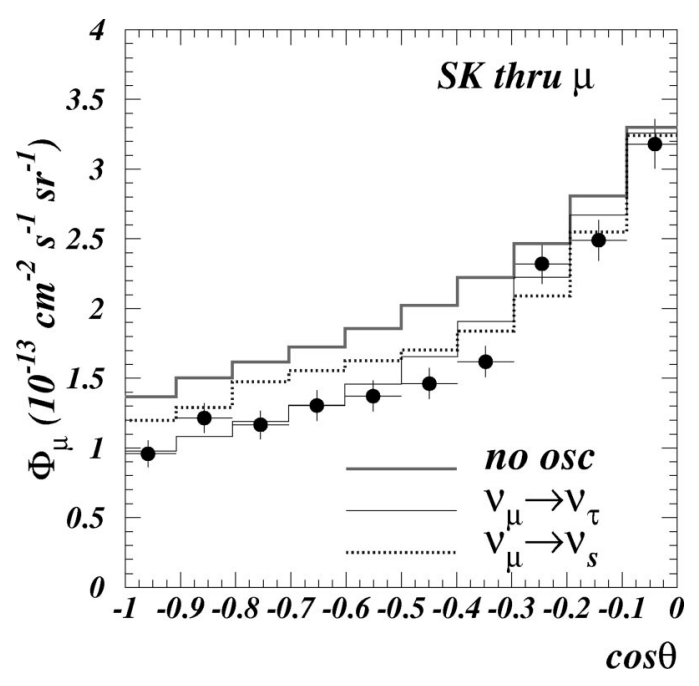

FIG. 30. Zenith-angle dependence for through-going muons at SuperKamiokande compared with the predictions in the case of no oscillation and for the best-fit points of $\nu_{\mu} \rightarrow \nu_{\tau}\left(\Delta m^{2}\right.$ $\left.=2.6 \times 10^{-3} \mathrm{eV}^{2}, \sin ^{2} 2 \theta=0.97\right)$ and $\nu_{\mu} \rightarrow \nu_{s} \quad\left(\Delta m^{2}=3 \times 10^{-3}\right.$ $\mathrm{eV}^{2}, \sin ^{2} \theta=0.61$ ) oscillations.

tween the $\nu_{\tau}$ and $\nu_{s}$ hypotheses for explaining atmospheric $\nu_{\mu}$ disappearance. One is to examine events likely to have been caused by neutral-current interactions. While $\nu_{\tau}$ 's readily undergo such interactions, $\nu_{s}$ 's do not, resulting in a relative suppression of the neutralcurrent signal (Hall and Murayama, 1998; Vissani and Smirnov, 1998). Another method attempts to observe the appearance of newly created $\nu_{\tau}$, even if only on a statistical basis, by selecting enriched samples. All methods strongly favor $\nu_{\mu} \leftrightarrow \nu_{\tau}$ oscillations over $\nu_{\mu} \leftrightarrow \nu_{S}$ (Fukuda et al., 2000; Toshito et al., 2001).

\section{LABORATORY EXPERIMENTS}

Laboratory experiments to search for neutrino oscillations are performed with neutrino beams produced at either accelerators or nuclear reactors. In disappearance experiments, using a neutrino beam primarily composed of a single flavor, one looks for attenuation due to mixing with other flavors. In appearance experiments, one searches for interactions by neutrinos of a flavor not present in the original neutrino beam.

Most of the past laboratory experiments did not have an oscillation signal, nor do most of the present ones. In such a case, as discussed in Sec. III.A, the experiment sets a limit on the corresponding oscillation probability. Appearance experiments set limits $\left\langle P_{\alpha \beta}\right\rangle\left\langle P_{L}\right.$ for given flavors $\alpha \neq \beta$. Disappearance experiments set limits $\left\langle P_{\alpha \alpha}\right\rangle>1-P_{L}$ for a given flavor $\alpha$ which, in the twoneutrino case, can be translated into $\left\langle P_{\alpha \beta}\right\rangle<P_{L}$ for $\beta$ $\neq \alpha$. The results are usually interpreted in a twoneutrino framework as exclusion regions in the $\left(\Delta m^{2}, \sin ^{2} 2 \theta\right)$ plane. One can take the upper bound on the mixing angle in the asymptotic large $\Delta m^{2}$ range and translate it back into the value of $P_{L}: \sin ^{2} 2 \theta_{\text {lim }}=2 P_{L}$ (see discussion in Sec. III.A). The probability $P_{L}$ is the relevant quantity when interpreting the results in the more-than-two-neutrino framework.

\section{A. Short-baseline experiments at accelerators}

Conventional neutrino beams from accelerators are mostly produced by $\pi$ decays, with the pions produced by the scattering of the accelerated protons on a fixed target:

$$
\begin{aligned}
& p+\text { target } \rightarrow \pi^{ \pm}+X, \\
& \pi^{ \pm} \rightarrow \mu^{ \pm}+\nu_{\mu}\left(\bar{\nu}_{\mu}\right), \\
& \mu^{ \pm} \rightarrow e^{ \pm}+\nu_{e}\left(\bar{\nu}_{e}\right)+\bar{\nu}_{\mu}\left(\nu_{\mu}\right) .
\end{aligned}
$$

Thus the beam can contain both $\mu$ and $e$ neutrinos and antineutrinos. The final composition and energy spectrum of the neutrino beam is determined by selecting the sign of the decaying $\pi$ and by stopping the produced $\mu$ in the beam line.

Most oscillation experiments performed so far with neutrino beams from accelerators have characteristic distances of the order of hundreds of meters. We call them short-baseline experiments. With the exception of the LSND experiment, which we discuss below, all searches have been negative. In Table IV we show the limits on the various transition probabilities from the negative results of the most restricting short-baseline experiments. As can be seen in the table, due to the short path length, these experiments are not sensitive to the low values of $\Delta m^{2}$ invoked to explain either the solar or the atmospheric neutrino data.

\section{B. LSND and KARMEN}

The only positive signature of oscillations in a laboratory experiment comes from the Liquid Scintillator Neutrino Detector (LSND) running at Los Alamos Meson Physics Facility (Athanassopoulos et al., 1995, 1996, 1998). The primary neutrino flux comes from $\pi^{+}$'s produced in a 30 -cm-long water target when hit by protons from the facility's linac with $800 \mathrm{MeV}$ kinetic energy. The detector is a tank filled with 167 metric tons of dilute liquid scintillator, located about $30 \mathrm{~m}$ from the neutrino source.

Most of the produced $\pi^{+}$'s come to rest and decay through the sequence $\pi^{+} \rightarrow \mu^{+} \nu_{\mu}$, followed by $\mu^{+}$ $\rightarrow e^{+} \nu_{e} \bar{\nu}_{\mu}$. The $\bar{\nu}_{\mu}$ 's so produced have a maximum energy of $52.8 \mathrm{MeV}$. This is called the decay at rest flux and is used to study $\bar{\nu}_{\mu} \rightarrow \bar{\nu}_{e}$ oscillations. The energy dependence of the $\bar{\nu}_{\mu}$ flux from decay at rest is very well known, and the absolute value is known to within $7 \%$. The open space around the target is short compared to the pion-decay length. Thus only 3\% of the $\pi^{+}$'s decay in flight. The decay in flight $\nu_{\mu}$ flux is used to study $\nu_{\mu}$ $\rightarrow \nu_{e}$ oscillations.

For decay-at-rest-related measurements, $\bar{\nu}_{e}$ 's are detected in the quasielastic process $\bar{\nu}_{e} p \rightarrow e^{+} n$, in correlation with a monochromatic photon of $2.2 \mathrm{MeV}$ arising from the neutron capture reaction $n p \rightarrow d \gamma$. The main 
TABLE IV. 90\% C.L. limit on the neutrino oscillation probabilities from negative searches at short-baseline experiments.

\begin{tabular}{|c|c|c|c|c|c|}
\hline Experiment & Beam & Channel & Limit (90\%) & $\Delta m_{\min }^{2}\left(\mathrm{eV}^{2}\right)$ & Ref. \\
\hline CDHSW & CERN & $\nu_{\mu} \rightarrow \nu_{\mu}$ & $P_{\mu \mu}>0.95$ & 0.25 & Dydak et al., 1984 \\
\hline E776 & $\mathrm{BNL}$ & $\nu_{\mu} \rightarrow \nu_{e}$ & $P_{e \mu}<1.5 \times 10^{-3}$ & 0.075 & Borodovsky et al., 1992 \\
\hline E734 & BNL & $\nu_{\mu} \rightarrow \nu_{e}$ & $P_{e \mu}<1.6 \times 10^{-3}$ & 0.4 & Ahrens et al., 1987 \\
\hline KARMEN2 & Rutherford & $\bar{\nu}_{\mu} \rightarrow \bar{\nu}_{e}$ & $P_{e \mu}<6.5 \times 10^{-4}$ & 0.05 & Wolf et al., 2001 \\
\hline E531 & FNAL & $\nu_{\mu} \rightarrow \nu_{\tau}$ & $P_{\mu \tau}<2.5 \times 10^{-3}$ & 0.9 & Ushida et al., 1986 \\
\hline \multirow[t]{3}{*}{ CCFR } & FNAL & $\nu_{\mu} \rightarrow \nu_{\tau}$ & $P_{\mu \tau}<4 \times 10^{-3}$ & 1.6 & McFarland et al., 1995 \\
\hline & & $\nu_{e} \rightarrow \nu_{\tau}$ & $P_{e \tau}<0.10$ & 20 & Naples et al., 1999 \\
\hline & & $\nu_{\mu} \rightarrow \nu_{e}$ & $P_{\mu e}<9 . \times 10^{-4}$ & 1.6 & Romosan et al., 1997 \\
\hline \multirow[t]{2}{*}{ Chorus } & CERN & $\nu_{\mu} \rightarrow \nu_{\tau}$ & $P_{\mu \tau}<3.4 \times 10^{-4}$ & 0.6 & Eskut et al., 2001 \\
\hline & & $\nu_{e} \rightarrow \nu_{\tau}$ & $P_{e \tau}<2.6 \times 10^{-2}$ & 7.5 & Eskut et al., 2001 \\
\hline \multirow[t]{3}{*}{ Nomad } & CERN & $\nu_{\mu} \rightarrow \nu_{\tau}$ & $P_{\mu \tau}<1.7 \times 10^{-4}$ & 0.7 & Astier et al., 2001 \\
\hline & & $\nu_{e} \rightarrow \nu_{\tau}$ & $P_{e \tau}<7.5 \times 10^{-3}$ & 5.9 & Astier et al., 2001 \\
\hline & & $\nu_{\mu} \rightarrow \nu_{e}$ & $P_{\mu e}<6 \times 10^{-4}$ & 0.4 & Valuev et al., 2001 \\
\hline
\end{tabular}

background is due to the $\bar{\nu}_{e}$ component in the beam that is produced in the decay chain starting with $\pi^{-}$s. This background is suppressed by three factors. First, the $\pi^{+}$ production rate is about eight times the $\pi^{-}$production rate in the beam stop. Second, $95 \%$ of the $\pi^{-}$'s come to rest and are absorbed before decay in the beam stop. Third, $88 \%$ of the $\mu^{-}$'s from $\pi^{-}$'s decay-in-flight are captured from atomic orbit, a process which does not give a $\bar{\nu}_{e}$. Thus, the relative yield, compared to the positive channel, is estimated to be $\sim(1 / 8) \times 0.05 \times 0.12$ $=7.5 \times 10^{-4}$. For decay-in-flight-related measurements, the $\nu_{e}$ 's are observed via the detection of electrons produced in the process $\nu_{e} C \rightarrow e^{-} X$ with energy $60<E_{e}$ $<200 \mathrm{MeV}$.

In both decay at rest and in flight measurements, an excess of events was observed as compared to the expected background while the excess was consistent with $\bar{\nu}_{\mu} \rightarrow \bar{\nu}_{e}$ oscillations. Further supporting evidence was provided by the signal in the $\nu_{\mu} \rightarrow \nu_{e}$ channel. In the latest results including the runs till 1998 (Aguilar et al., 2001 ) the total fitted excess is of $87.9 \pm 22.4 \pm 6$ events, corresponding to an oscillation probability of (2.64 $\pm 0.67 \pm 0.45) \times 10^{-3}$. In the two-family formalism these results lead to the oscillation parameters shown in Fig. 31. The shaded regions are the $90 \%$ and $99 \%$ likelihood regions from LSND. The best-fit point corresponds to $\Delta m^{2}=1.2 \mathrm{eV}^{2}$ and $\sin ^{2} 2 \theta=0.003$.

The region of parameter space which is favored by the LSND observations has been partly tested by other experiments like the old BNL E776 experiment (Borodovsky et al., 1992) and more recently by the KARMEN experiment (Gemmeke et al., 1990). The KARMEN experiment is performed at the neutron spallation facility ISIS of the Rutherford Appleton Laboratory. Neutrinos are produced by stopping the $800 \mathrm{MeV}$ protons in a massive beam stop target, thereby producing pions. The $\pi^{-}$'s are absorbed by the target nuclei whereas $\pi^{+}$'s decay at rest producing muon neutrinos via $\pi^{+} \rightarrow \mu^{+} \nu_{\mu}$. The low momentum $\mu^{+}$s are also stopped within the massive target and decay at rest, $\mu^{+} \rightarrow e^{+} \nu_{e} \bar{\nu}_{\mu}$. The $\pi^{+}-\mu^{+}$decay chain at rest gives a neutrino source with identical intensities for $\nu_{\mu}, \nu_{e}$, and $\bar{\nu}_{\mu}$ emitted isotropically. There is a minor fraction of $\pi^{-}$decaying in flight with the following $\mu^{-}$decay at rest in the target station which leads to a very small contamination of $\bar{\nu}_{e} / \nu_{e}$ $<6.2 \times 10^{-4}$. The energy spectra of the $\nu$ 's are well defined due to the decay at rest of both the $\pi^{+}$and $\mu^{+}$.

The neutrinos are detected in a rectangular tank filled with $56 \mathrm{t}$ of a liquid scintillator. The signature for the detection of $\bar{\nu}_{e}$ is a spatially correlated delayed coincidence of positrons from $\bar{p}\left(\bar{\nu}_{e}, e^{+}\right) n$ with energies up to $E_{e^{+}}=E_{\bar{\nu}_{e}}-Q=51.0 \mathrm{MeV}$ and $\gamma$ emission of either of

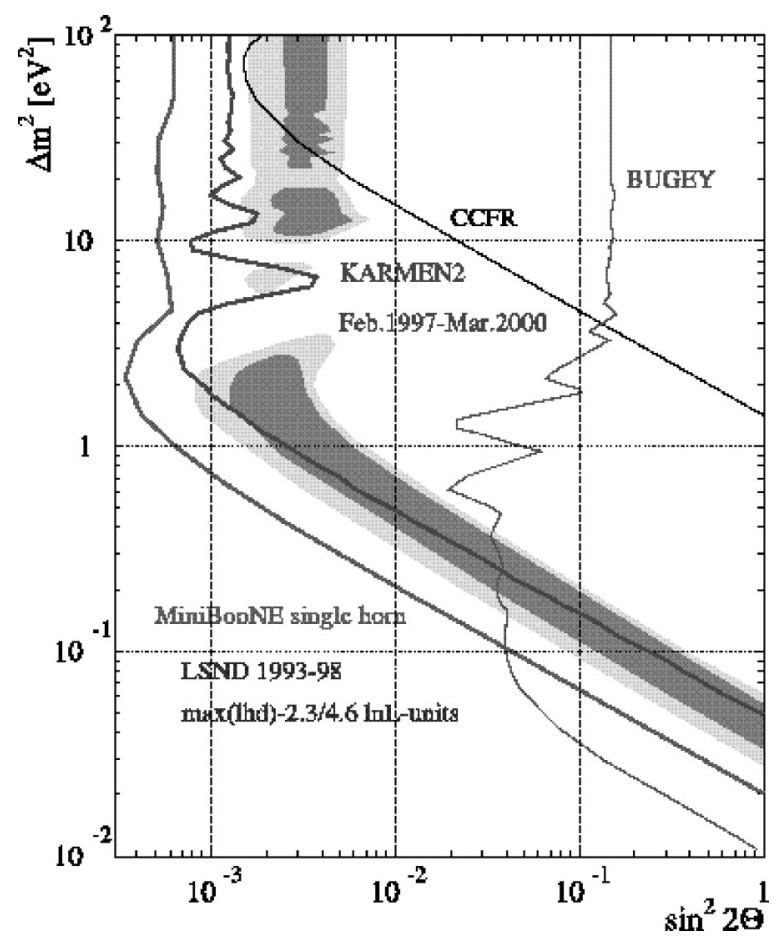

FIG. 31. Allowed regions (at 90\% and 99\% C.L.) for $\nu_{e} \rightarrow \nu_{\mu}$ oscillations from the LSND experiment compared with the exclusion regions (at 90\% C.L.) from KARMEN2 and other experiments. The $90 \%$ C.L. expected sensitivity curve for MiniBooNE is also shown. From Wolf et al., 2001. 
the two neutron capture processes: $p(n, \gamma) d$ with one $\gamma$ of $E(\gamma)=2.2 \mathrm{MeV}$ or $\operatorname{Gd}(n, \gamma)$ with $3 \gamma$ quanta on average and $\Sigma E(\gamma)=8 \mathrm{MeV}$.

The raw data presented at the EPS HEP 2001 conference (Wolf et al., 2001) correspond to $\sim 9400 \mathrm{C}$ protons on target. An analysis of the data results in 11 sequential events which satisfy all cuts. This number is in good agreement with the total background expectation of 12.3 \pm 0.6. Applying a Bayesian renormalization procedure, an upper limit of $N($ osc $)<6.3$ at $90 \%$ C.L. can be extracted. However, using the spectral information and a maximum-likelihood analysis, KARMEN find a best-fit value $N(\mathrm{osc})=0$ within the physically allowed range of parameters, which can be translated into an upper limit of 3.8 and 3.1 oscillation events for $\Delta m^{2}<1 \mathrm{eV}^{2}$ and $\Delta m^{2}>20 \mathrm{eV}^{2}$, respectively. These numbers are based on a complete frequentist approach as suggested by $\mathrm{G}$. Feldman and R. Cousins. The corresponding exclusion curve in the two-neutrino parameter space is given in Fig. 31 together with the favored region for the LSND experiment (from Wolf et al., 2001). At high $\Delta m^{2}$, KARMEN results exclude the region favored by LSND. At low $\Delta m^{2}$, KARMEN leaves some allowed space, but the reactor experiments at Bugey and $\mathrm{CHOOZ}$ add stringent limits for the larger mixing angles. This figure represents the final status of the LSND oscillation signal.

The MiniBooNE experiment (Bazarko et al., 2000) searches for $\nu_{\mu} \rightarrow \nu_{e}$ oscillations and is specially designed to make a conclusive statement about the LSND's neutrino oscillation evidence. They use a $\nu_{\mu}$ beam of energy $0.5-1.0 \mathrm{GeV}$ initiated by a primary beam of $8-\mathrm{GeV}$ protons from the Fermilab Booster, which contains only a small intrinsic $\nu_{e}$ component (less than $0.3 \%$ ). They search for an excess of electron neutrino events in a detector located approximately $500 \mathrm{~m}$ from the neutrino source. The MiniBooNE neutrino detector consists of 800 tons of pure mineral oil contained in a $12.2-\mathrm{m}$ diameter spherical tank. A structure in the tank supports phototubes, which detect neutrino interactions in the oil by the Cerenkov and scintillation light that they produce.

The $L / E$ ratio is similar to that of LSND, giving MiniBooNE sensitivity to the same mode of oscillations. However, neutrino energies are more than an order of magnitude higher than at LSND, so that the search at MiniBooNE employs different experimental techniques. In Fig. 31 we show the $90 \%$ C.L. limits that MiniBooNE can achieve. Should a signal be found then the next step would be the BooNe experiment.

\section{Disappearance experiments at reactors}

Neutrino oscillations are also searched for using neutrino beams from nuclear reactors. Nuclear reactors produce $\bar{\nu}_{e}$ beams with $E_{\nu} \sim \mathrm{MeV}$. Due to the low energy, $e$ 's are the only charged leptons which can be produced in the neutrino charged-current interaction. If the $\bar{\nu}_{e}$ oscillated to another flavor, its charged-current interaction could not be observed. Therefore oscillation experiments performed at reactors are disappearance experi-

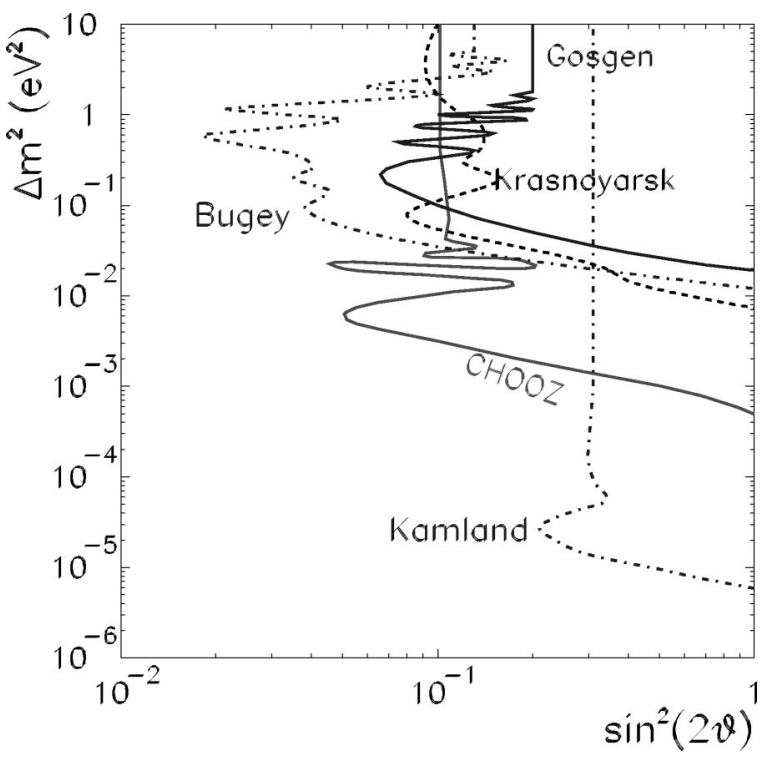

FIG. 32. Excluded regions at $90 \%$ C.L. for $\nu_{e}$ oscillations from reactors experiments and the expected sensitivity from the KamLAND experiment.

ments. They have the advantage that smaller values of $\Delta m^{2}$ can be accessed due to the lower neutrino beam energy.

In Fig. 32 we show the corresponding excluded regions in the parameter space for two neutrino oscillations from the negative results of the reactor experiments Gosgen (Zacek, 1986), Krasnoyarsk (Vidyakin et al., 1994), Bugey (Achkar et al., 1995), and CHOOZ (Apollonio et al., 1999). Gosgen, Krasnoyarks, and Bugey have relatively short baselines. From the figure we see that Bugey sets the strongest constraint on the allowed mixing in the $\Delta m^{2}$ range that is interesting for the LSND signal. CHOOZ, which can be considered the first long baseline reactor experiment $(L \simeq 1 \mathrm{~km})$, is sensitive to lower values of $\Delta m^{2}$. Its $90 \%$ C.L. limits include $\Delta m^{2}<7 \times 10^{-4} \mathrm{eV}^{2}$ for maximal mixing, and $\sin ^{2} 2 \theta<0.10$ for large $\Delta m^{2}$. The CHOOZ results are significant in excluding part of the region that corresponds to the large-mixing-angle solution of the solar neutrino problem (see Sec. IV). Furthermore, the CHOOZ bound rules out with high significance the possibility that $\nu_{\mu} \rightarrow \nu_{e}$ oscillations explain the atmospheric neutrino deficit. The $\mathrm{CHOOZ}$ constraint on the mixing angle is also relevant to the interpretation of the atmospheric neutrino anomaly in the framework of threeneutrino mixing. We return to this issue in Sec. V.

Smaller values of $\Delta m^{2}$ can be accessed at future reactor experiments using longer baseline. Pursuing this idea, the KamLAND experiment (Piepke et al., 2001), a 1000-ton liquid scintillation detector, is currently in operation in the Kamioka mine in Japan. This underground site is conveniently located at a distance of 150 $210 \mathrm{~km}$ from several Japanese nuclear power stations. The measurement of the flux and energy spectrum of the $\bar{\nu}_{e}$ 's emitted by these reactors will provide a test to the large-mixing-angle solution of the solar neutrino anomaly. In Fig. 32 we plot the expected $90 \%$ sensitivity 
for the KamLAND experiment after three years of data taking (from Piepke et al., 2001). The experiment will, for the first time, provide a completely solar-modelindependent test of this particle physics solution of the solar neutrino problem. After a few years of data taking, it should be capable of either excluding the entire largemixing-angle region or, not only establishing $\nu_{e} \leftrightarrow \nu_{\text {other }}$ oscillations, but also measuring the oscillation parameters with unprecedented precision. Data taking is expected to commence in 2002.

\section{Long-baseline experiments at accelerators}

Smaller values of $\Delta m^{2}$ can also be accessed using accelerator beams at long-baseline experiments. In these experiments the intense neutrino beam from an accelerator is aimed at a detector located underground at a distance of several hundred kilometers. The main goal of these experiments is to test the presently allowed solution for the atmospheric neutrino problem by searching for either $\nu_{\mu}$ disappearance or $\nu_{\tau}$ appearance. At present there are three such projects approved: K2K (Nishikawa et al., 1997; Ahn et al., 2001; Nishikawa, et al., 2001), which runs with a baseline of about $235 \mathrm{~km}$ from KEK to SK, MINOS (Ables et al., 1995; Wojcicki, 2001) under construction with a baseline of $730 \mathrm{~km}$ from Fermilab to the Soudan mine where the detector will be placed, and OPERA (Shibuya et al., 1997; Cocco et al., 2000), under construction with a baseline of $730 \mathrm{~km}$ from CERN to Gran Sasso. With their expected sensitivities, these experiments can cover either some fraction or all of the parameter region suggested by the atmospheric neutrino anomaly discussed in Sec. V.

In the $\mathrm{K} 2 \mathrm{~K}$ experiment, a wide-band, almost pure $\nu_{\mu}$ beam from $\pi^{+}$decays is generated in the KEK $12-\mathrm{GeV} / \mathrm{c}$ Proton Synchrotron and a neutrino beam line. The detector is in SK at a distance of $250 \mathrm{~km}$. Various beam monitors along the beam line and two different types of front detectors have also been constructed at the KEK site. The front detectors are a $1 \mathrm{kt}$ water Cerenkov detector, which is a miniature of the SK detector, and a so-called fine-grained detector which is composed of a scintillating fiber tracker trigger counters, lead glass counters and a muon range detector. The characteristics of the neutrino beam in the KEK sitedirection, intensity, stability, energy spectrum and $\nu_{e}$ $-\nu_{\mu}$ composition are examined using front detectors and beam monitors. They are then extrapolated to the SK site and used to obtain the expected number of events and the energy spectrum.

The K2K experiment had a successful start in early 1999, and data were recorded during several periods in 2000 and 2001. The accumulated beam intensity during the 2000 runs was $22.9 \times 10^{18}$ protons on target (Ahn et al., 2001), increased to $\sim 38 \times 10^{18}$ protons on target with the 2001 run till the summer (Nishikawa et al., 2001), which was about $40 \%$ of the goal of the experiment, $10^{20}$ protons on target. The no-oscillation prediction for this sample, based on the data from the front detectors, is $63.9_{-6.6}^{+6.1}$ events, while a total of 44 events have been observed in $22.5 \mathrm{kt}$ of the fiducial volume. The statistical probability that the observation would be equal to or smaller than 44 is $\sim 8 \%$. In the presence of oscillations the expected number of events would be 41.5 for $\Delta m^{2}=3 \times 10^{-3} \mathrm{eV}^{2}$ and maximal mixing. Although the central value of the number of observed events is consistent with the data from atmospheric neutrinos, the discrepancy with the no-oscillation prediction is still within the statistical error. $\mathrm{K} 2 \mathrm{~K}$ has also studied the energy distribution of these events compared to the expectation based on the pion monitor data and a Monte Carlo simulation, and finds good agreement with the expectation from oscillations with the atmospheric mass difference and maximal mixing, but the statistics are too poor to give any further constraint.

MINOS is designed to detect neutrinos delivered by the Main Injector accelerator at Fermilab (NuMI) with average energies of $\sim 5-15 \mathrm{GeV}$ depending on the beam configuration. Two detectors, functionally identical, will be placed in the NuMI neutrino beam: one at Fermilab and the second one in Soudan iron mine, 732 $\mathrm{km}$ away. The MINOS detectors are iron/scintillator sampling calorimeters with a toroidal magnetic field in the iron. Observed interactions of $\nu_{\mu}$ can be divided into two classes: charged-current-like, with an identified $\mu$ track, and NC-like, muonless. The ratio of the observed numbers of the charged-current- and neutral-currentlike events in the two detectors provides a sensitive test for oscillations. With its expected sensitivity, MINOS will be able to precisely measure (roughly at the level of $10 \%$ ) the oscillation parameters in the $\nu_{\mu} \rightarrow \nu_{\tau}$ channel. The primary measurement for this is the comparison of the rate and spectrum of the charged-current events in the Far Detector with those in the Near Detector. Comparing the neutral-current/charged-current ratios in the two detectors, the experiment can also be sensitive to the presence of $\nu_{\mu} \rightarrow \nu_{s}$ oscillations. MINOS is scheduled to start data taking at the end of 2004.

Both $\mathrm{K} 2 \mathrm{~K}$ and MINOS have also the capability for detecting the appearance of $\nu_{e}$ 's due to $\nu_{\mu} \rightarrow \nu_{e}$ oscillations. This signal however suffers from large backgrounds due to both the $\nu_{e}$ 's in the beam and the NC events with a topology similar to the $\nu_{e}$ interaction. These backgrounds can be partially suppressed using the information from the near detectors. In particular, MINOS may be able to improve the CHOOZ bounds.

OPERA is designed to search for $\nu_{\mu} \rightarrow \nu_{\tau}$ oscillations in the Gran Sasso Laboratory. It will study the interaction of $20-\mathrm{GeV}$ neutrinos produced at CERN. The goal is to observe the appearance of $\nu_{\tau}{ }^{\prime} \mathrm{s}$ in a pure $\nu_{\mu}$ beam. The detector is based on a massive lead/nuclear emulsion target. Nuclear emulsions are exploited for the direct observation of the decay of the $\tau$ in a very low background environment.

\section{E. Direct determination of neutrino masses}

Oscillation experiments have provided us with important information on the differences between the neu- 
trino masses-squared, $\Delta m_{i j}^{2}$, and on the leptonic mixing angles, $U_{i j}$. But they are insensitive to the absolute mass scale for the neutrinos, $m_{i}$.

Of course, the results of an oscillation experiment do provide a lower bound on the heavier mass in $\Delta m_{i j}^{2}$, $\left|m_{i}\right| \geqslant \sqrt{\Delta m_{i j}^{2}}$ for $\Delta m_{i j}^{2}>0$. But there is no upper bound on this mass. In particular, the corresponding neutrinos could be approximately degenerate at a mass scale that is much higher than $\sqrt{\Delta m_{i j}^{2}}$. Moreover, there is neither upper nor lower bound on the lighter mass $m_{j}$.

Information on the neutrino masses, rather than mass differences, can be extracted from kinematic studies of reactions in which a neutrino or an antineutrino is involved. In the absence of mixing, the present limits are (Groom et al., 2000)

$$
\begin{aligned}
& m_{\nu_{\tau}}<18.2 \mathrm{MeV}\left(95 \% \text { C.L.) from } \tau^{-} \rightarrow n \pi+\nu_{\tau},\right. \\
& m_{\nu_{\mu}}<190 \mathrm{keV} \text { (90\% C.L.) from } \pi^{-} \rightarrow \mu^{-}+\bar{\nu}_{\mu},
\end{aligned}
$$

$$
m_{\nu_{e}}<2.2 \mathrm{eV}\left(95 \% \text { C.L.) from }{ }^{3} \mathrm{H} \rightarrow{ }^{3} \mathrm{He}+e^{-}+\bar{\nu}_{e}\right. \text {, }
$$

where for the bound on $m_{v_{e}}$ we take the latest limit from the Mainz experiment (Bonn et al., 2001). A similar bound is obtained by the Troitsk experiment (Lobashev et al., 2001). A new experimental project, KATRIN, is under consideration with an estimated sensitivity limit: $m_{\nu_{e}} \sim 0.3 \mathrm{eV}$.

In the presence of mixing these limits have to be modified and in general they involve more than a single flavor parameter. The limit that is most relevant to our purposes is the most sensitive one from tritium beta decay. In presence of mixing, the electron neutrino is a combination of mass eigenstates and the tritium beta decay spectrum is modified as (Shrock, 1980)

$\frac{d N}{d E}=R(E) \sum_{i}\left|U_{e i}\right|^{2}\left[\left(E_{0}-E\right)^{2}-m_{i}^{2}\right]^{1 / 2} \Theta\left(E_{0}-E-m_{i}\right)$,

where $E$ is the energy of electron, $E_{0}$ is the total decay energy, and $R(E)$ is $m_{\nu}$ independent. The step function, $\Theta\left(E_{0}-E-m_{i}\right)$, reflects the fact that a given neutrino can only be produced if the available energy is larger than its mass. According to Eq. (126), there are two important effects, sensitive to the neutrino masses and mixings, on the electron energy spectrum: (i) kinks at the electron energies $E_{e}^{(i)}=E \sim E_{0}-m_{i}$ with sizes that are determined by $\left|U_{e i}\right|^{2}$; (ii) a shift of the end point to $E_{\mathrm{ep}}=E_{0}-m_{1}$, where $m_{1}$ is the lightest neutrino mass. The situation simplifies considerably if we are interested in constraining the possibility of quasidegenerate neutrinos with mass $\sim m_{\nu}$. In this case the distortion of the spectrum can be described by a single parameter (Farzan, Peres, and Smirnov, 2001; Vissani, 2001a), $m_{\beta}$ $=\Sigma_{i} m_{i}\left|U_{e i}\right|^{2} / \Sigma_{i}\left|U_{e i}\right|^{2} \sim m_{\nu}$. So the limit in Eq. (125) applies to the unique neutrino mass scale.

Direct information on neutrino masses can also be obtained from neutrinoless double beta decay $(2 \beta 0 \nu)$ searches $(A, Z) \rightarrow(A, Z+2)+e^{-}+e^{-}$. The rate of this process is proportional to the effective Majorana mass of $\nu_{e}$,

$$
m_{e e}=\left|\sum_{i} m_{i} U_{e i}^{2}\right|,
$$

which, in addition to five parameters that affect the tritium beta decay spectrum, depends also on the three leptonic $C P$-violating phases. Notice that in order to induce the $2 \beta 0 \nu$ decay, $\nu_{e}$ must be a Majorana particle.

The present strongest bound from $2 \beta 0 \nu$ decay is obtained by the Heidelberg-Moscow group (KlapdorKleingrothaus et al., 2001):

$$
m_{e e}<0.34(0.26) \mathrm{eV}, \quad 90 \% \text { (68\%) C.L. }
$$

Taking into account systematic errors related to nuclear matrix elements, the bound may be weaker by a factor of about 3. A sensitivity of $m_{e e} \sim 0.1 \mathrm{eV}$ is expected to be reached by the currently running NEMO3 experiment (Marquet et al., 2000), while a series of new experiments (CUORE, EXO, GENIUS) is planned with sensitivity of up to $m_{e e} \sim 0.01 \mathrm{eV}$.

The knowledge of $m_{e e}$ will provide information on the mass and mixing parameters that is independent of the $\Delta m_{i j}^{2}$ s. However, to infer the values of neutrino masses, additional assumptions are required. In particular, the mixing elements are complex and may lead to strong cancellation, $m_{e e} \ll m_{1}$. Yet, the combination of results from $2 \beta 0 \nu$ decays and tritium beta decay can test and, in some cases, determine the mass parameters of given schemes of neutrino masses (Vissani, 1999; Bilenky, Pascoli, and Petcov, 2001a, 2001b; Farzan, Peres, and Smirnov, 2001; Klapdor-Kleingrothaus, Pas, and Smirnov, 2001).

\section{THREE-NEUTRINO MIXING}

In the previous sections we discussed the three pieces of evidence for neutrino masses and mixing (solar neutrinos, atmospheric neutrinos, and the LSND results) as usually formulated in the framework of two-neutrino oscillations. The results are summarized in Fig. 33 where we show the ranges of masses and mixing implied by these signals at $90 \%$ and $99 \%$ C.L. for 2 degrees of freedom, as well as relevant constraints from negative searches in laboratory experiments.

The three pieces of evidence correspond to three values of mass-squared differences of different orders of magnitude. Consequently, there is no consistent explanation for all three signals based on oscillations among the three known neutrinos. The argument supporting this statement is very simple. With three neutrinos, there are only two independent mass-squared differences, since the following relation must hold:

$$
\Delta m_{21}^{2}+\Delta m_{32}^{2}+\Delta m_{13}^{2}=0 \text {. }
$$

This relation cannot be satisfied by three $\Delta m_{i j}^{2}$ that are of different orders of magnitude. One may wonder if this naive extrapolation from the two-neutrino oscilla- 


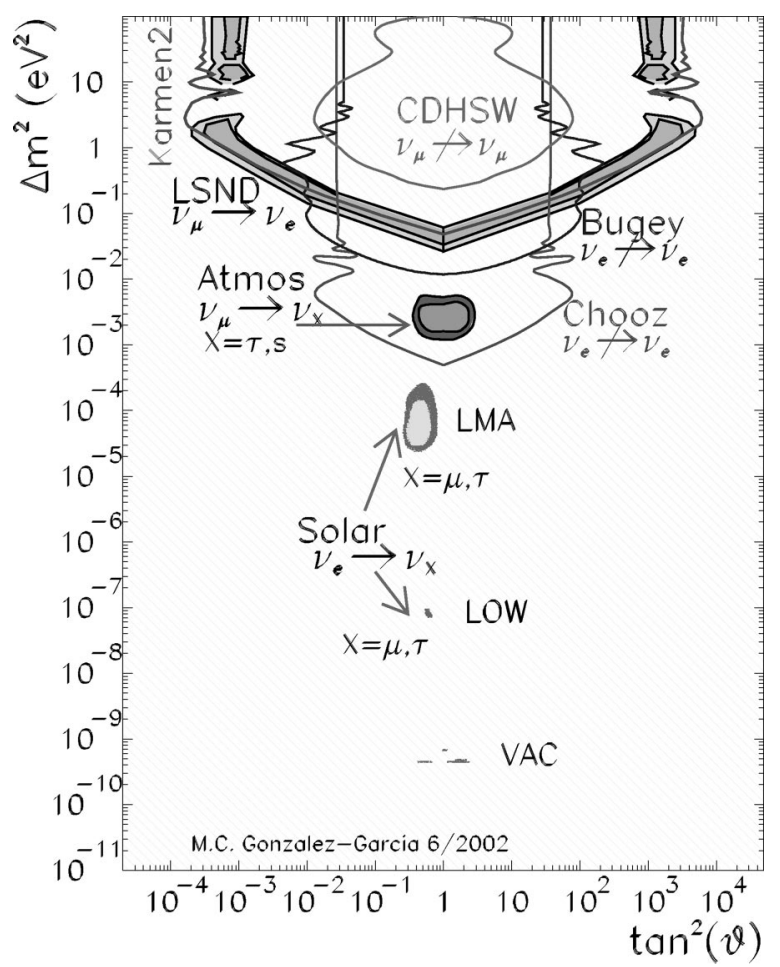

FIG. 33. Summary of the present pieces of evidence for neutrino masses and mixing as obtained from $2-\nu$ oscillation analyses. The allowed regions correspond to $90 \%$ and $99 \%$ C.L. for 2 degrees of freedom. We also show relevant bounds from laboratory experiments.

tion picture holds once the full mixing structure of a three-neutrino oscillation is taken into account or, conversely, once some special configuration of the threeneutrino parameters fits the three pieces of evidence. The combined fit to the data performed by Fogli, Lisi, Marrone, and Scioscia (1999a) and by Gonzalez-Garcia and Maltoni (2002) shows that this is not the case.

Whereas in the case of the solar and atmospheric neutrino indications several experiments agree on the existence of the effect, the third indication is presently found only by the LSND experiment. In many studies the LSND result is therefore left out and the analysis of the solar and atmospheric data is performed in the framework of mixing between the three known neutrinos. We follow this approach and discuss next the derived masses and mixing in these scenarios. Alternatively, a possible explanation of the four pieces of evidence with only three neutrinos has been proposed assuming that $C P T$ is violated in the neutrino sector (see, for instance, Barenboim, Borissov, Lykken, and Smirnov, 2001; Murayama and Yanagida, 2001).

The combined description of both solar and atmospheric anomalies requires that all three known neutrinos take part in the oscillations. The mixing parameters are encoded in the $3 \times 3$ lepton mixing matrix (Maki, Nakagawa, and Sakata, 1962; Kobayashi and Maskawa, 1973). The two Majorana phases do not affect neutrino oscillations (Bilenky, Hosek, and Petcov, 1980; Langacker et al., 1987). The Dirac phase (that is, the analog of the KM phase of the quark sector) does affect neu- trino oscillations in general, but for the purposes of this section we can set it to zero.

In this case the mixing matrix can be conveniently parametrized in the standard form (Groom et al., 2000):

$$
U=\left(\begin{array}{ccc}
c_{13} c_{12} & s_{12} c_{13} & s_{13} \\
-s_{12} c_{23}-s_{23} s_{13} c_{12} & c_{23} c_{12}-s_{23} s_{13} s_{12} & s_{23} c_{13} \\
s_{23} s_{12}-s_{13} c_{23} c_{12} & -s_{23} c_{12}-s_{13} s_{12} c_{23} & c_{23} c_{13}
\end{array}\right),
$$

where $c_{i j} \equiv \cos \theta_{i j}$ and $s_{i j} \equiv \sin \theta_{i j}$.

As we have seen in the previous sections, in most of the parameter space of solutions for solar and atmospheric oscillations, the required mass differences satisfy

$$
\Delta m_{\odot}^{2} \ll \Delta m_{\text {atm }}^{2} .
$$

In this approximation the angles $\theta_{i j}$ can be taken without loss of generality to lie in the first quadrant, $\theta_{i j}$ $\in[0, \pi / 2]$. There are two possible mass orderings which we chose as

$$
\begin{aligned}
& \Delta m_{21}^{2}=\Delta m_{\odot}^{2} \ll \Delta m_{32}^{2} \simeq \Delta m_{31}^{2}=\Delta m_{\mathrm{atm}}^{2}>0 ; \\
& \Delta m_{21}^{2}=\Delta m_{\odot}^{2} \ll-\Delta m_{31}^{2} \simeq-\Delta m_{32}^{2}=\left|\Delta m_{\mathrm{atm}}^{2}\right|>0 .
\end{aligned}
$$

We refer to the first option, Eq. (132), as the direct scheme, and to the second one, Eq. (133), as the inverted scheme. The direct scheme is naturally related to hierarchical masses, $m_{1} \ll m_{2} \ll m_{3}$, for which $m_{2} \simeq \sqrt{\Delta m_{21}^{2}}$ and $m_{3} \simeq \sqrt{\Delta m_{32}^{2}}$, or to quasidegenerate masses, $m_{1} \simeq m_{2}$ $\simeq m_{3} \gg \Delta m_{21}^{2}, \Delta m_{32}^{2}$. On the other hand, the inverted scheme implies that $m_{3}<m_{1} \simeq m_{2}$.

One may wonder how good an approximation it is to set the $C P$-violating phases to zero. It turns out to be an excellent approximation for the analysis of solar, atmospheric, and laboratory data if Eq. (131) holds. In this case, as discussed below, no simultaneous effect of the two mass differences is observable in any $\nu$-appearance transition.

\section{A. Probabilities}

The determination of the oscillation probabilities for both solar and atmospheric neutrinos requires that one solves the evolution equation of the neutrino system in the matter background of the Sun or the Earth. In a three-flavor framework, this equation reads

$$
i \frac{d \vec{\nu}}{d t}=H \vec{\nu}, \quad H=U \cdot H_{0}^{d} \cdot U^{\dagger}+V,
$$

where $U$ is the lepton mixing matrix, $\vec{\nu} \equiv\left(\nu_{e}, \nu_{\mu}, \nu_{\tau}\right)^{T}$, $H_{0}^{d}$ is the vacuum Hamiltonian,

$$
H_{0}^{d}=\frac{1}{2 E_{\nu}} \operatorname{diag}\left(-\Delta m_{21}^{2}, 0, \Delta m_{32}^{2}\right)
$$

and $V$ is the effective potential that describes chargedcurrent forward interactions in matter:

$$
V=\operatorname{diag}\left( \pm \sqrt{2} G_{F} N_{e}, 0,0\right) \equiv \operatorname{diag}\left(V_{e}, 0,0\right) .
$$

In Eq. (136), the sign $+(-)$ refers to neutrinos (antineutrinos), and $N_{e}$ is electron number density in the Sun or the Earth. 
In what follows we focus on the direct scheme of Eq. (132), for which the five relevant parameters are related to experiments in the following way:

$$
\begin{aligned}
& \Delta m_{\odot}^{2}=\Delta m_{21}^{2}, \quad \Delta m_{\text {atm }}^{2}=\Delta m_{32}^{2}, \\
& \theta_{\odot}=\theta_{12}, \quad \theta_{\text {atm }}=\theta_{23}, \quad \theta_{\text {reactor }}=\theta_{13} .
\end{aligned}
$$

For transitions in vacuum, the results apply also to the inverted scheme of Eq. (133). In the presence of matter effects, the direct and inverted schemes are no longer equivalent, although the difference is hardly recognizable in the current solar and atmospheric neutrino phenomenology as long as Eq. (131) holds (Fogli, Lisi, Montanino, and Scioscia, 1997; Gonzalez-Garcia and Maltoni, 2002). Under this approximation, the results obtained for the direct scheme can be applied to the inverted scheme by replacing $\Delta m_{32}^{2} \rightarrow-\Delta m_{32}^{2}$.

In general the transition probabilities present an oscillatory behavior with two oscillation lengths. However, the hierarchy in the splittings, Eq. (131), leads to important simplifications.

Let us first consider the analysis of solar neutrinos. A first simplification occurs because $L_{32}^{\text {osc }}=4 \pi E / \Delta m_{32}^{2}$ is much shorter than the distance between the Sun and the Earth. Consequently, the oscillations related to $L_{32}^{\text {osc }}$ are averaged in the evolution from the Sun to the Earth. A second simplification occurs because, for the evolution in both the Sun and the Earth, $\Delta m_{32}^{2}$ $\gg 2 \sqrt{2} G_{F} N_{e} E_{\nu} \sin ^{2} 2 \theta_{13}$. Consequently, matter effects on the evolution of $\nu_{3}$ can be neglected. The result of these two approximations is that the three-flavor evolution equations decouple into an effective two-flavor problem for the basis (Kuo and Pantaleone, 1986; Shi and Schramm, 1992)

$$
\nu_{e^{\prime}}=c_{12} \nu_{1}+s_{12} \nu_{2}, \quad \nu_{\mu^{\prime}}=-s_{12} \nu_{1}+c_{12} \nu_{2},
$$

with the substitution of $N_{e}$ by the effective density

$$
N_{e} \Rightarrow N_{e} \cos ^{2} \theta_{13} \text {. }
$$

Thus the survival probability takes the following form:

$$
P_{e e, \odot}^{3 \nu}=\sin ^{4} \theta_{13}+\cos ^{4} \theta_{13} P_{e^{\prime} e^{\prime}, \odot}^{2 \nu},
$$

where $P_{e^{\prime} e^{\prime}, \odot}^{2 \nu}$ is the two-flavor survival probability in the $\left(\Delta m_{21}^{2}, \theta_{12}\right)$ parameter space, but with the modified matter density of Eq. (140). We conclude that the analysis of the solar data constrains three of the five independent oscillation parameters: $\Delta m_{21}^{2}, \theta_{12}$, and $\theta_{13}$.

Equation (141) reveals the dominant effect of a nonvanishing $\theta_{13}$ in the solar neutrino survival probability: the energy-dependent part of the probability, $P_{e^{\prime} e^{\prime}, \odot}^{2 \nu}$, gets damped by the factor $\cos ^{4} \theta_{13}$, while an energyindependent term, $\sin ^{4} \theta_{13}$, is added. Thus increasing $\theta_{13}$ makes the solar neutrino survival probability more and more energy independent.

Let us now consider the analysis of atmospheric neutrinos. Here $L_{21}^{\text {osc }}=4 \pi E / \Delta m_{21}^{2}$ is much larger than the relevant distance scales. Consequently, the corresponding oscillating phase is negligible. In this approximation one can rotate away the corresponding angle $\theta_{12}$. Thus the resulting survival probabilities do not depend on $\Delta m_{21}^{2}$ and $\theta_{12}$. For instance, for constant Earth matter density, the various $P_{\alpha \beta}$ can be written as follows:

$$
\begin{aligned}
P_{e e}=1 & -4 s_{13, m}^{2} c_{13, m}^{2} S_{31}, \\
P_{\mu \mu}=1 & -4 s_{13, m}^{2} c_{13, m}^{2} s_{23}^{4} S_{31}-4 s_{13, m}^{2} s_{23}^{2} c_{23}^{2} S_{21} \\
& -4 c_{13, m}^{2} s_{23}^{2} c_{23}^{2} S_{32}, \\
P_{\tau \tau}=1 & -4 s_{13, m}^{2} c_{13, m}^{2} c_{23}^{4} S_{31}-4 s_{13, m}^{2} s_{23}^{2} c_{23}^{2} S_{21} \\
& -4 c_{13, m}^{2} s_{23}^{2} c_{23}^{2} S_{32}, \\
P_{e \mu}= & 4 s_{13, m}^{2} c_{13, m}^{2} s_{23}^{2} S_{31}, \\
P_{e \tau}= & 4 s_{13, m}^{2} c_{13, m}^{2} c_{23}^{2} S_{31}, \\
P_{\mu \tau}= & -4 s_{13, m}^{2} c_{13, m}^{2} s_{23}^{2} c_{23}^{2} S_{31}+4 s_{13, m}^{2} s_{23}^{2} c_{23}^{2} S_{21} \\
& +4 c_{13, m}^{2} s_{23}^{2} c_{23}^{2} S_{32} .
\end{aligned}
$$

Here $\theta_{13, m}$ is the effective mixing angle in matter,

$\sin 2 \theta_{13, m}=\frac{\sin 2 \theta_{13}}{\sqrt{\left(\cos 2 \theta_{13}-2 E_{\nu} V_{e} / \Delta m_{32}^{2}\right)^{2}+\left(\sin 2 \theta_{13}\right)^{2}}}$,

and $S_{i j}$ are the oscillating factors in matter,

$$
S_{i j}=\sin ^{2}\left(\frac{\Delta \mu_{i j}^{2}}{4 E_{\nu}} L\right) .
$$

In Eq. (144), $\Delta \mu_{i j}^{2}$ are the effective mass-squared differences in matter:

$$
\begin{aligned}
& \Delta \mu_{21}^{2}=\frac{\Delta m_{32}^{2}}{2}\left(\frac{\sin 2 \theta_{13}}{\sin 2 \theta_{13, m}}-1\right)-E_{\nu} V_{e}, \\
& \Delta \mu_{32}^{2}=\frac{\Delta m_{32}^{2}}{2}\left(\frac{\sin 2 \theta_{13}}{\sin 2 \theta_{13, m}}+1\right)+E_{\nu} V_{e}, \\
& \Delta \mu_{31}^{2}=\Delta m_{32}^{2} \frac{\sin 2 \theta_{13}}{\sin 2 \theta_{13, m}},
\end{aligned}
$$

and $L$ is the path length of the neutrino within the Earth, which depends on its direction. We conclude that the analysis of the atmospheric data constrains three of the five independent oscillation parameters: $\Delta m_{32}^{2}, \theta_{23}$, and $\theta_{13}$.

So we find that, in the approximation of Eq. (131), the mixing angle $\theta_{13}$ is the only parameter common to both solar and atmospheric neutrino oscillations and which may potentially allow for some mutual influence. The main effect of the three-neutrino mixing is that now atmospheric neutrinos can oscillate simultaneously in both the $\nu_{\mu} \rightarrow \nu_{\tau}$ and $\nu_{\mu} \rightarrow \nu_{e}$ (and, similarly, $\nu_{e} \rightarrow \nu_{\tau}$ and $\nu_{e}$ $\rightarrow \nu_{\mu}$ ) channels. The oscillation amplitudes for channels involving $\nu_{e}$ are controlled by the size of $\sin ^{2} \theta_{13}$ $=\left|U_{e 3}\right|^{2}$. We learn that in the approximation of Eq. (131), solar and atmospheric neutrino oscillations decouple in the limit $\theta_{13}=0$. This angle is constrained by the $\mathrm{CHOOZ}$ reactor experiment. To analyze the CHOOZ constraints we need to evaluate the survival probability for $\bar{\nu}_{e}$ of average energy $E \sim$ few $\mathrm{MeV}$ at a 
distance of $L \sim 1 \mathrm{~km}$. For these values of energy and distance, one can safely neglect Earth matter effects. The survival probability takes the analytical form

$$
\begin{aligned}
P_{e e}^{\mathrm{CHOOZ}=} & 1-\cos ^{4} \theta_{13} \sin ^{2} 2 \theta_{12} \sin ^{2}\left(\frac{\Delta m_{21}^{2} L}{4 E_{\nu}}\right) \\
& -\sin ^{2} 2 \theta_{13}\left[\cos ^{2} \theta_{12} \sin ^{2}\left(\frac{\Delta m_{31}^{2} L}{4 E_{\nu}}\right)\right. \\
& \left.+\sin ^{2} \theta_{12} \sin ^{2}\left(\frac{\Delta m_{32}^{2} L}{4 E_{\nu}}\right)\right] \\
\simeq & 1-\sin ^{2} 2 \theta_{13} \sin ^{2}\left(\frac{\Delta m_{32}^{2} L}{4 E_{\nu}}\right),
\end{aligned}
$$

where the second equality holds under the approximation $\Delta m_{21}^{2} \ll E_{\nu} / L$ which can only be safely made for $\Delta m_{21}^{2} \lesssim 3 \times 10^{-4} \mathrm{eV}^{2}$. Thus in general the analysis of the $\mathrm{CHOOZ}$ reactor date involves four oscillation parameters: $\Delta m_{32}^{2}, \theta_{13}, \Delta m_{21}^{2}$, and $\theta_{12}$ (Bilenky, Nicolo, and Petcov, 2001; Gonzalez-Garcia and Maltoni, 2002).

\section{B. Allowed masses and mixing}

There are several three-neutrino oscillation analyses in the literature which include either solar (Fogli, Lisi, and Montanino, 1996; Fogli, Lisi, Montanino, and Palazzo, 2000a, 2000b; Gago, Nunokawa, and Zukanovich Funchal, 2001) or atmospheric (Fogli, Lisi, Montanino, and Scioscia, 1997; Fogli, Lisi, Marrone, and Scioscia, 1999b; Teshima and Sakai, 1999; de Rujula, Gavela, and Hernandez, 2001; Fogli, Lisi, and Marrone, 2001) neutrino data. Combined studies have also been performed (Barger, Whistnant, and Phillips, 1980; Fogli, Lisi, and Montanino, 1994, 1995; Barger and Whisnant, 1999). We follow and update here the results from the latest analysis of Gonzalez-Garcia, Maltoni, Peña-Garay, and Valle (2001).

As discussed above, in the approximation of Eq. (131), solar and atmospheric neutrino oscillations decouple in the limit $\left|U_{e 3}\right|=\sin \theta_{13}=0$. In this limit, the allowed values of the parameters can be obtained directly from the results of the analyses in terms of twoneutrino oscillations presented in Secs. IV and V. Deviations from the two-neutrino scenario are then determined by the size of $\theta_{13}$. Thus the first question to answer is how the presence of this additional angle affects the analysis of the solar and atmospheric neutrino data.

In Fig. 34 we show the allowed regions for the oscillation parameters $\Delta m_{21}^{2}$ and $\tan ^{2} \theta_{12}$ from the global analysis of the solar neutrino data in the framework of three-neutrino oscillations for different values of $\sin ^{2} \theta_{13}$ (updated from Gonzalez-Garcia, Maltoni, PeñaGaray, and Valle, 2001). The allowed regions for a given confidence level are defined as the set of points satisfying the condition $\chi^{2}\left(\Delta m_{12}^{2}, \tan ^{2} \theta_{12}, \tan ^{2} \theta_{13}\right)-\chi_{\min }^{2}$ $\leqslant \Delta \chi^{2}$ (C.L. 3 degrees of freedom) where, for instance, $\Delta \chi^{2}($ C.L. 3 degrees of freedom $)=6.25,7.83$, and 11.36

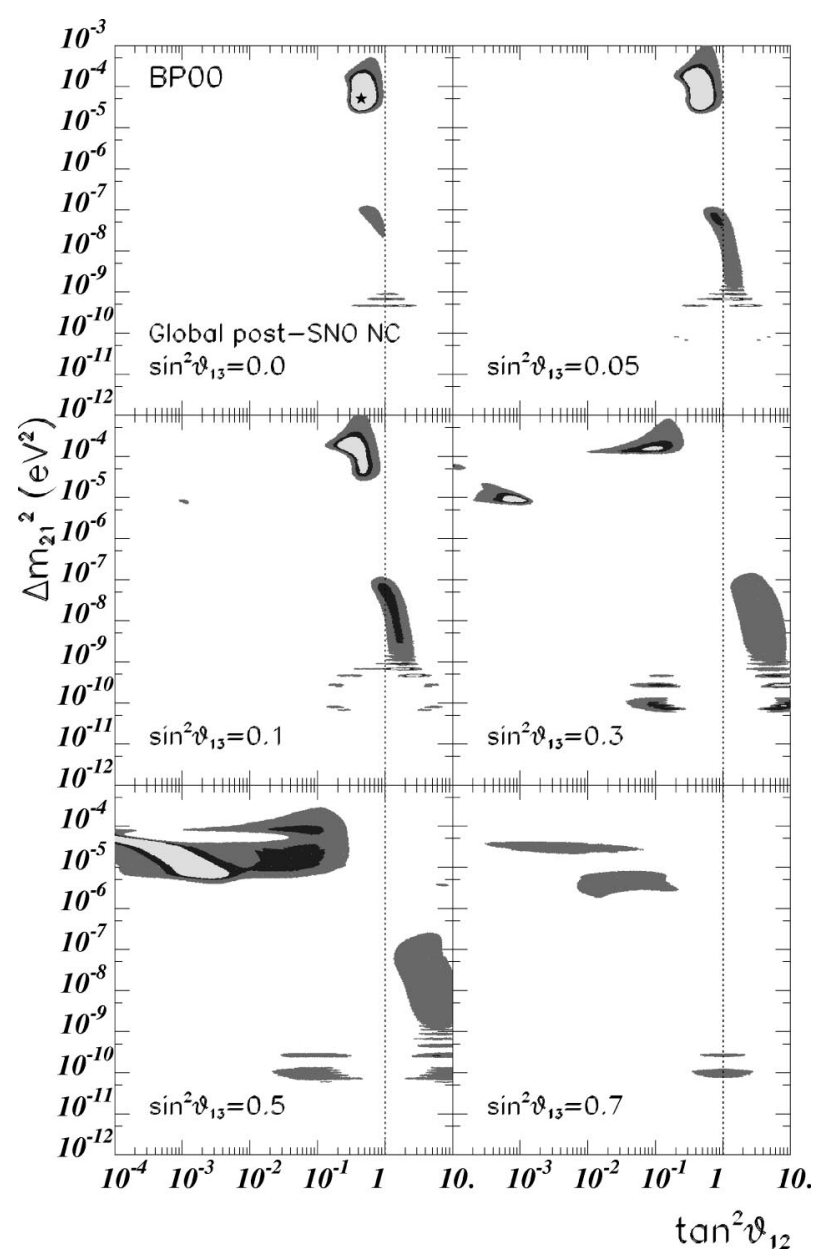

FIG. 34. Allowed regions (at 90\%, 95\%, and 99\% C.L.) in the $\left(\Delta m_{21}^{2}, \tan ^{2} \theta_{12}\right)$ plane from a global analysis of solar neutrino data in the framework of three-neutrino oscillations for various values of $\sin ^{2} \theta_{13}$. The global best-fit point is marked by the star.

for C.L. $=90 \%, 95 \%$, and 99\%, respectively. The global minimum used in the construction of the regions lies in the large-mixing-angle region and corresponds to $\tan ^{2} \theta_{13}=0$, that is, to the decoupled scenario.

As seen in the figure, the modifications to the decoupled case are significant only if $\theta_{13}$ is large. As $\sin ^{2} \theta_{13}$ increases, all the allowed regions disappear, leading to an upper bound on $\sin ^{2} \theta_{13}$ that is independent of the values taken by the other parameters in the threeneutrino mixing matrix. For instance, no region of parameter space is allowed (at 99\% C.L. for 3 degrees of freedom) for $\sin ^{2} \theta_{13}=\left|U_{e 3}\right|^{2}>0.80$. This fact is also illustrated in Fig. 35 where we plot the shift in $\chi^{2}$ as a function of $\sin ^{2} \theta_{13}$ when the mass and mixing parameters $\Delta m_{12}^{2}$ and $\tan ^{2} \theta_{12}$ are chosen to minimize $\chi^{2}$.

In Fig. 36 we show the allowed regions for the oscillation parameters $\Delta m_{32}^{2}$ and $\tan ^{2} \theta_{23}$ from the analysis of the atmospheric neutrino data in the framework of three-neutrino oscillations for different values of $\sin ^{2} \theta_{13}$ (updated from Gonzalez-Garcia, Maltoni, Peña-Garay, and Valle, 2001). In the upper-left panel $\tan ^{2} \theta_{13}=0$, which corresponds to pure $\nu_{\mu} \rightarrow \nu_{\tau}$ oscillations. Note the 


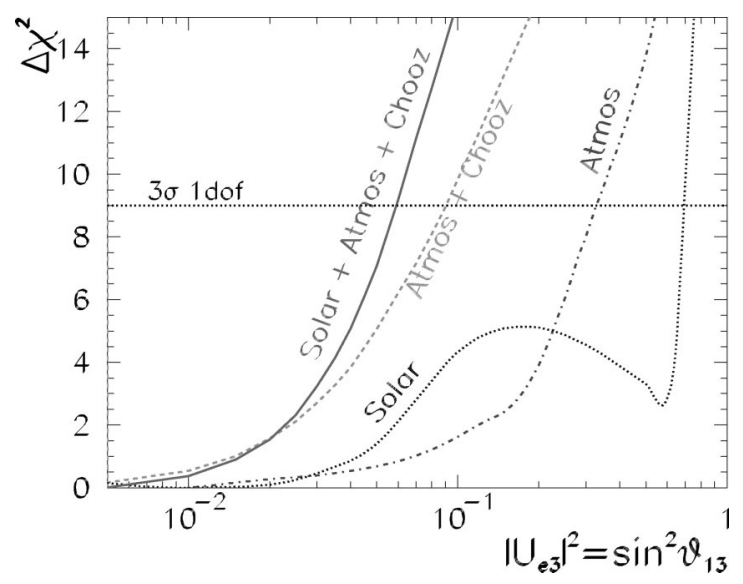

FIG. 35. Dependence of $\Delta \chi^{2}$ on $\sin ^{2} \theta_{13}$ in the analysis of the atmospheric, solar, and $\mathrm{CHOOZ}$ neutrino data. The dotted horizontal line corresponds to the $3 \sigma$ limit for a single parameter.

exact symmetry of the contour regions under the transformation $\theta_{23} \rightarrow \pi / 4-\theta_{23}$. This symmetry follows from the fact that in the pure $\nu_{\mu} \rightarrow \nu_{\tau}$ channel, matter effects cancel out and the oscillation probability depends on $\theta_{23}$ only through the double-valued function $\sin ^{2} 2 \theta_{23}$ (see Sec. V.C). For $\theta_{13} \neq 0$ this symmetry breaks due to the three-neutrino mixing structure even if matter effects are neglected. The analysis of the full atmospheric neutrino data in the framework of three-neutrino oscilla-

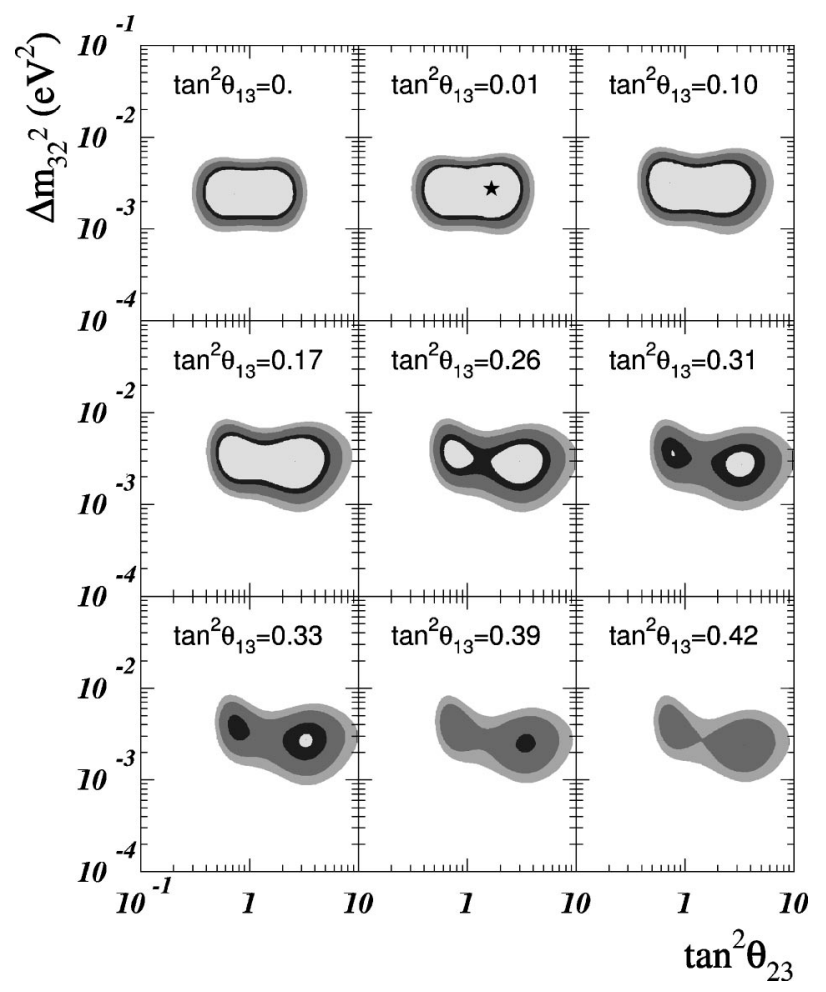

FIG. 36. Allowed regions (at 90\%, 95\%, 99\%, and 99.7\% C.L. $)$ in the $\left(\Delta m_{32}^{2}, \tan ^{2} \theta_{23}\right)$ plane from a global analysis of the atmospheric neutrino data in the framework of three-neutrino oscillations for various values of $\tan ^{2} \theta_{13}$. The global best-fit point is marked by the star. tions clearly favors the $\nu_{\mu} \rightarrow \nu_{\tau}$ oscillation hypothesis. As a matter of fact, the best fit corresponds to a very small value, $\theta_{13}=6^{\circ}$, but it is statistically indistinguishable from the decoupled scenario, $\theta_{13}=0^{\circ}$. No region of parameter space is allowed (at $99 \%$ C.L. for 3 degrees of freedom) for $\sin ^{2} \theta_{13}=\left|U_{e 3}\right|^{2}>0.40$. The physics reason for this limit is clear from the discussion of the $\nu_{\mu} \rightarrow \nu_{e}$ oscillation channel in Sec. V.C: large values of $\theta_{13}$ imply too large a contribution of the $\nu_{\mu} \rightarrow \nu_{e}$ channel and would spoil the otherwise successful description of the angular distribution of contained events. This situation is illustrated also in Fig. 35 where we plot the shift of $\chi^{2}$ for the analysis of atmospheric data in the framework of oscillations between three neutrinos as a function of $\sin ^{2} \theta_{13}$ when the mass and mixing parameters $\Delta m_{32}^{2}$ and $\tan ^{2} \theta_{23}$ are chosen to minimize $\chi^{2}$.

For any value of the mixing parameters, the masssquared difference relevant for the atmospheric analysis is restricted to lie in the interval $1.25 \times 10^{-3}$ $<\Delta m_{32}^{2} / \mathrm{eV}^{2}<8 \times 10^{-3}$ at $99 \%$ C.L. Thus it is within the range of sensitivity of the $\mathrm{CHOOZ}$ experiment. Indeed, as illustrated in Fig. 35, the limit on $\sin ^{2} \theta_{13}$ becomes stronger when the $\mathrm{CHOOZ}$ data are combined with the atmospheric and solar neutrino results.

One can finally perform a global analysis in the fivedimensional parameter space combining the full set of solar, atmospheric, and reactor data. Such analysis leads to the following allowed $3 \sigma$ ranges for individual parameters (that is, when the other four parameters have been chosen to minimize the global $\chi^{2}$ ):

$2.4 \times 10^{-5}<\Delta m_{21}^{2} / \mathrm{eV}^{2}<2.4 \times 10^{-4} \quad$ large mixing angle, $0.27<\tan ^{2} \theta_{12}<0.77$ large mixing angle,

$1.4 \times 10^{-3}<\Delta m_{32}^{2} / \mathrm{eV}^{2}<6.0 \times 10^{-3}$,

$0.4<\tan ^{2} \theta_{23}<3.0$,

$\sin ^{2} \theta_{13}<0.06$.

These results can be translated into our present knowledge of the moduli of the mixing matrix $U$ :

$$
|U|=\left(\begin{array}{ccc}
0.73-0.89 & 0.45-0.66 & <0.24 \\
0.23-0.66 & 0.24-0.75 & 0.52-0.87 \\
0.06-0.57 & 0.40-0.82 & 0.48-0.85
\end{array}\right) .
$$

In conclusion, we learn that at present the large mixingtype solutions provide the best fit. As concerns $\left|U_{e 3}\right|$, both solar and atmospheric data favor small values, and this trend is strengthened by the reactor data.

\section{IMPLICATIONS OF THE NEUTRINO MASS SCALE AND FLAVOR STRUCTURE}

\section{A. New physics}

The simplest and most straightforward lesson of the evidence for neutrino masses is also the most striking one: there is new physics beyond the standard model. This is the first experimental result that is inconsistent with the standard model. 
Most likely, the new physics is related to the existence of singlet fermions at some high energy scale that induce, at low energies, the effective terms Eq. (13) through the seesaw mechanism. The existence of heavy singlet fermions is predicted by many extensions of the standard model, especially by GUT's [beyond SU(5)] and left-right symmetric theories.

There are of course other possibilities. One could induce neutrino masses without introducing any new fermions beyond those of the standard model. This would require the existence of a scalar $\Delta_{L}(1,3)_{+1}$, that is, a triplet of $\mathrm{SU}(2)_{L}$. The smallness of neutrino masses would then be related to the smallness of the vacuum expectation value $\left\langle\Delta_{L}^{0}\right\rangle$ (required also by the success of the $\rho=1$ relation) and would not have a generic natural explanation.

In left-right symmetric models, however, where the breaking of $\mathrm{SU}(2)_{R} \times \mathrm{U}(1)_{B-L} \rightarrow \mathrm{U}(1)_{Y}$ is induced by the vacuum expectation value of an $\mathrm{SU}(2)_{R}$ triplet, $\Delta_{R}$, there must exist also an $\mathrm{SU}(2)_{L}$ triplet scalar. Furthermore, the Higgs potential leads to an order-ofmagnitude relation between the various vacuum expectation values $\left\langle\Delta_{L}^{0}\right\rangle\left\langle\Delta_{R}^{0}\right\rangle \sim v^{2}$ (where $v$ is the electroweak breaking scale), and the smallness of $\left\langle\Delta_{L}^{0}\right\rangle$ is related to the high scale of $\mathrm{SU}(2)_{R}$ breaking. This situation can be thought of as a seesaw of vacuum expectation values. In this model there are, however, also singlet fermions. The light neutrino masses arise from both the seesaw mechanism and the triplet vacuum expectation value.

Neutrino masses could also be of the Dirac type. Here, again, singlet fermions have to be introduced, but lepton number conservation needs to be imposed by hand. This possibility is disfavored by theorists since it is likely that global symmetries are violated by gravitational effects. Furthermore, the lightness of neutrinos (compared to charged fermions) is again unexplained.

Another possibility is that neutrino masses are generated by mixing with singlet fermions but the mass scale of these fermions is not high. Here again the lightness of neutrino masses remains a puzzle. The best known example of such a scenario is the framework of supersymmetry without $R$ parity.

Let us emphasize that the seesaw mechanism or, more generally, the extension of the standard model with nonrenormalizable terms, is the simplest explanation of neutrino masses. Models in which neutrino masses are produced by new physics at low energy imply a much more dramatic modification of the standard model. Furthermore, the existence of seesaw masses is an unavoidable prediction of various extensions of the standard model. In contrast, many (but not all) of the low-energy mechanisms are introduced for the specific purpose of generating neutrino masses.

In this and in the next section, where we discuss the implications of the experimental data for theories beyond the standard model, we choose to focus on models that explain the atmospheric and solar neutrino data through mixing among three active neutrinos. In other words, we assume three-neutrino mixing with the oscillation parameters derived in Sec. VII. We do not review models that try to incorporate the LSND data by adding light sterile neutrinos and only comment on this possibility in the context of theories with new extra dimensions.

\section{B. The scale of new physics}

Given Eq. (14), $m_{\nu} \sim v^{2} / \Lambda_{\mathrm{NP}}$, it is straightforward to use measured neutrino masses to estimate the scale of new physics that is relevant to their generation. In particular, if there is no quasidegeneracy in the neutrino masses, the heaviest of the active neutrino masses can be estimated,

$$
m_{h}=m_{3} \sim \sqrt{\Delta m_{\text {atm }}^{2}} \approx 0.05 \mathrm{eV} .
$$

(In the case of an inverted hierarchy the implied scale is $m_{h}=m_{2} \sim \sqrt{\left|\Delta m_{\mathrm{atm}}^{2}\right|} \approx 0.05 \mathrm{eV}$.) It follows that the scale in the nonrenormalizable term (13) is given by

$$
\Lambda_{\mathrm{NP}} \sim v^{2} / m_{h} \approx 10^{15} \mathrm{GeV} \text {. }
$$

We should clarify two points regarding Eq. (150):

(1) There could be some level of degeneracy between the neutrino masses that is relevant to atmospheric neutrino oscillations. In such a case (see Sec. VI.E), Eq. (149) is modified into a lower bound and, consequently, Eq. (150) becomes an upper bound on the scale of the new physics.

(2) It could be that the $Z_{i j}$ couplings of Eq. (13) are much smaller than 1. In such a case, again, Eq. (150) becomes an upper bound on the scale of the new physics. On the other hand, in models of approximate flavor symmetries, there are relations between the structures of the charged lepton and neutrino mass matrices that give quite generically $Z_{33} \geq m_{\tau}^{2} / v^{2} \sim 10^{-4}$. We conclude that the likely range of $\Lambda_{\mathrm{NP}}$ that is implied by the atmospheric neutrino results is given by

$$
10^{11} \lesssim \Lambda_{\mathrm{NP}} \lesssim 10^{15} \mathrm{GeV} \text {. }
$$

The estimates (150) and (151) are very exciting. First, the upper bound on the scale of the new physics is well below the Planck scale. This means that there is a new scale in Nature which is intermediate between the two known scales, the Planck scale, $m_{\mathrm{P1}} \sim 10^{19} \mathrm{GeV}$, and the electroweak breaking scale, $v \sim 10^{2} \mathrm{GeV}$.

It is amusing to note in this regard that the solar neutrino problem does not necessarily imply such a new scale. If its solution is related to vacuum oscillations with $\Delta m_{21}^{2} \sim 10^{-10} \mathrm{eV}^{2}$, it can be explained by $\Lambda_{\mathrm{NP}} \sim m_{\mathrm{Pl}}$. However (see Sec. IV.D.4), the favored explanation for the solar neutrino deficit is the large-mixing-angle solution, which again points towards a new physics scale in the range of Eq. (151).

Second, the scale $\Lambda_{\mathrm{NP}} \sim 10^{15} \mathrm{GeV}$ is intriguingly close to the scale of gauge coupling unification. We shall say more about this fact when we discuss GUT's.

\section{Implications for flavor physics}

1. The flavor parameters

Flavor physics refers to interactions that are not universal in generation space. In the standard-model inter- 
action basis, this is the physics of the Yukawa couplings. In the mass basis, this term refers to fermion masses and mixing parameters. There are two main reasons for the interest in flavor physics:

(1) The standard model has 13 flavor parameters. These are the nine charged-fermion masses or, equivalently, Yukawa couplings, $Y_{i}=m_{i} / v / \sqrt{2}$,

$$
\begin{aligned}
& Y_{t} \sim 1, \quad Y_{c} \sim 10^{-2}, \quad Y_{u} \sim 10^{-5}, \\
& Y_{b} \sim 10^{-2}, \quad Y_{s} \sim 10^{-3}, \quad Y_{d} \sim 10^{-4}, \\
& Y_{\tau} \sim 10^{-2}, \quad Y_{\mu} \sim 10^{-3}, \quad Y_{e} \sim 10^{-6},
\end{aligned}
$$

and the four CKM parameters,

$$
\left|V_{u s}\right| \sim 0.2,\left|V_{c b}\right| \sim 0.04,\left|V_{u b}\right| \sim 0.004, \sin \delta_{\mathrm{KM}} \sim 1 .
$$

One can easily see that the flavor parameters are hierarchical (that is, they have very different magnitudes from each other), and all but two (the top Yukawa and the $C P$-violating phase) are small. The unexplained smallness and hierarchy pose the flavor puzzle of the standard model. Its solution may direct us to physics beyond the standard model.

(2) The smallness of flavor-changing neutral-current (FCNC) processes, such as $\Delta m_{K}$ and $\mu \rightarrow e \gamma$, is explained within the standard model by the GlashowIliopoulos-Maiani (GIM) mechanism. In many extensions of the standard model there is, generically, no such mechanism. In some cases, experimental bounds on flavor-changing processes are violated. The solution of such flavor problems leads to refinement of the models. In other cases, the model predictions are within present bounds but well above the standard-model range. We can hope, then, to probe this new physics through future measurements of flavor-changing processes.

Many mechanisms have been proposed in response to either or both of these flavor aspects. For example, approximate horizontal symmetries, broken by a small parameter, can lead to selection rules that both explain the hierarchy of the Yukawa couplings and suppress flavorchanging couplings in sectors of new physics.

In the extension of the standard model with three active neutrinos that have Majorana-type masses, there are nine new flavor parameters in addition to those of the standard model with massless neutrinos. These are three neutrino masses, three lepton mixing angles, and three phases in the mixing matrix. The counting of parameters is explained in Sec. II.D. Of these, four are determined from existing measurements of solar and atmospheric neutrino fluxes: two mass-squared differences and two mixing angles. This adds significantly to the input data on flavor physics and provides an opportunity to test and refine flavor models.

If neutrino masses arise from effective terms of the form of Eq. (13), then the overall scale of neutrino masses is related to the scale $\Lambda_{\mathrm{NP}}$ and, in most cases, does not tell us anything about flavor physics. More significant information for flavor models can be written in terms of three dimensionless parameters whose values can be read from the results of the global analysis in Eq. (147): First, the mixing angles that are relevant to atmospheric neutrinos,

$$
\left|U_{\mu 3} U_{\tau 3}^{*}\right| \sim 0.4-0.5
$$

second, the mixing angles that are relevant to solar neutrinos,

$$
\left|U_{e 1} U_{e 2}^{*}\right| \sim 0.35-0.5
$$

third, the ratio between the respective mass-squared differences,

$$
\frac{\Delta m_{21}^{2}}{\left|\Delta m_{32}^{2}\right|} \sim \begin{cases}10^{-1}-10^{-3} & \text { large mixing angle } \\ 10^{-4}-10^{-5} & \text { low mass. }\end{cases}
$$

In addition, the upper bound on the third mixing angle from the $\mathrm{CHOOZ}$ experiments often plays a significant role in flavor model building:

$$
\left|U_{e 3}\right| \lesssim 0.24 \text {. }
$$

\section{Special features of the neutrino flavor parameters}

There are several features in the numerical values of these parameters that have drawn much attention and have driven numerous investigations:

(i) Large mixing and strong hierarchy: The mixing angle that is relevant to the atmospheric neutrino problem, $U_{\mu 3}$, is large, of order one. On the other hand, the ratio of mass-squared differences $\Delta m_{21}^{2} /\left|\Delta m_{32}^{2}\right|$ is small. If there is no degeneracy in the neutrino sector then the small ratio of mass-squared differences implies a small ratio between the masses themselves, $m_{2} / m_{3} \ll 1$. (In the case of inverted hierarchy, the implied hierarchy is $m_{3} / m_{2} \ll 1$.) It is difficult to explain in a natural way a situation where, in the 2-3-generation sector, there is large mixing but the corresponding masses are hierarchical. Below we discuss in detail the difficulties and various possible solutions.

(ii) If the large-mixing-angle solution to the solar neutrino problem holds, then the data can be interpreted in a very different way. In this case, the two measured mixing angles are of order one. Moreover, $\Delta m_{21}^{2} /\left|\Delta m_{32}^{2}\right|$ $\sim 10^{-2}$ means that the ratio between the masses themselves (which, for fermions, are after all the fundamental parameters) is not very small, $m_{2} / m_{3} \sim 10^{-1}$. Such a value could easily be an accidentally small number, without any parametric suppression. If this is the correct way of reading the data, the measured neutrino parameters may actually reflect the absence of any hierarchical structure in the neutrino mass matrices [Hall, $\mathrm{Mu}$ rayama, and Weiner (2000); Berger and Siyeon (2001); Haba and Murayama (2001); Hirsch and King (2001)]. Obviously, this interpretation is plausible only if the large-mixing-angle solution to the solar neutrino problem holds, and will be excluded if the small-mixing-angle or low-mass-ratio solutions hold. Another important test of this idea will be provided by a measurement of $\left|U_{e 3}\right|$. If indeed the entries in $M_{\nu}$ have random values of the same order, all three mixing angles are expected to be of order one. If experiments measure $\left|U_{e 3}\right| \sim 10^{-1}$, that is, 
close to the present $\mathrm{CHOOZ}$ bound, it can be again argued that its smallness is accidental. The stronger the upper bound on this angle becomes, the more difficult it will be to maintain this view.

(iii) A special case of large mixing is that of maximal mixing. In a two-generation case, with a single mixing angle, maximal mixing is defined as $\sin ^{2} 2 \theta=1$. In the three-generation case, what we mean by maximal mixing is that the disappearance probability is equivalent to that for maximal two-neutrino mixing at the relevant mass scale (Barger et al., 1998b). Maximal atmospheric neutrino mixing corresponds to $4\left|U_{\mu 3}\right|^{2}\left(1-\left|U_{\mu 3}\right|^{2}\right)=1$, which leads to

$$
\left|U_{\mu 3}\right|^{2}=1 / 2 \text {. }
$$

Maximal solar neutrino mixing corresponds to $4\left|U_{e 1} U_{e 2}\right|^{2}=1$, which leads to

$$
\left|U_{e 1}\right|^{2}=\left|U_{e 2}\right|^{2}=1 / 2,\left|U_{e 3}\right|^{2}=0 .
$$

As can be seen in Sec. VII, the present data are consistent with near-maximal atmospheric neutrino mixing and large but not maximal solar neutrino mixing. The possibility that both mixings are near maximal is, however, not entirely excluded. This scenario, in which both atmospheric and solar neutrino mixing are near maximal, means that the structure of the leptonic mixing matrix is given to a good approximation by

$$
U=\left(\begin{array}{ccc}
\frac{1}{\sqrt{2}} & -\frac{1}{\sqrt{2}} & 0 \\
\frac{1}{2} & \frac{1}{2} & -\frac{1}{\sqrt{2}} \\
\frac{1}{2} & \frac{1}{2} & \frac{1}{\sqrt{2}}
\end{array}\right) .
$$

The case of Eq. (160) is commonly called bimaximal mixing. We should like to make the following comments, regarding maximal or bimaximal mixing:

(a) Theoretically, it is not difficult to construct models that explain near-maximal solar neutrino mixing in a natural way. We shall encounter some examples below. Experimentally, it may be difficult to make a convincing case for near-maximal (rather than just order-one) solar neutrino mixing (GonzalezGarcia, Peña-Garay, Nir, and Smirnov, 2001).

(b) It is highly nontrivial to construct models that explain near-maximal atmospheric neutrino mixing in a natural way. The reason is that near-maximal mixing is often related to quasidegeneracy. So when solar neutrino data are also taken into account, approximate degeneracy among all three neutrinos is required, and models of three quasidegenerate neutrinos are, in general, not easy to construct in a natural way.

(c) The case of bimaximal mixing is also very challenging for theory. Many of the attempts in the literature involve fine-tuning. (Alternatively, the term bimaximal mixing is sometimes used to denote the case of two large, rather than maximal, mixing angles.)

\section{Large mixing and strong hierarchy}

In this subsection, we focus on the large-mixingstrong-hierarchy problem. We explain the challenge to theory from this feature and present various solutions that have been proposed to respond to this challenge.

A large mixing angle by itself should not be a surprise. After all, the naive guess would be that all dimensionless couplings [such as the $Z_{i j}$ couplings of Eq. (13)] are naturally of order one and consequently all mixing angles are of order one. However, the quark mixing angles are small, and this situation has led to a prejudice that the lepton mixing angles would also be so. Second, and more importantly, flavor models have a built-in mechanism for naturally inducing smallness and hierarchy in the quark parameters (and perhaps also in charged-lepton masses). For example, a mechanism that has been intensively studied in the literature is that of selection rules due to an approximate symmetry. Within such frameworks, numbers of order one are as difficult (or as easy) to account for as small numbers: one can assign charges in such a way that small flavor parameters correspond to terms in the Lagrangian that carry charges different from zero, while the order-one parameters correspond to terms that carry no charge.

The combination of a large mixing angle and strong hierarchy is, however, a true puzzle. To understand the difficulty in this combination, let us assume that both the mixing and the hierarchy can be understood from the relevant $2 \times 2$ block in the mass matrix for light (Majorana) neutrinos. The generic form of this block is

$$
M_{\nu}=\frac{v^{2}}{\Lambda_{\mathrm{NP}}}\left(\begin{array}{ll}
A & B \\
B & C
\end{array}\right) .
$$

This matrix would lead to a large mixing angle if

$$
|B| \geq|C-A|,
$$

and to a strong mass hierarchy if (for simplicity we assume real entries)

$$
\left|A C-B^{2}\right| \ll \max \left(A^{2}, B^{2}, C^{2}\right) .
$$

If we examine the two-neutrino sector alone, these conditions mean fine-tuning. Hence the challenge is to find models in which the hierarchy is naturally induced.

To make the problem even sharper, let us explicitly consider models with a horizontal $\mathrm{U}(1)_{H}$ symmetry (Froggatt and Nielsen, 1979). A horizontal symmetry is one in which different generations may carry different charges, in contrast to the standard-model gauge group. We assume that the symmetry is broken by a small parameter $\lambda$. To be concrete, we take $\lambda=0.2$, close to the value of the Cabibbo angle. One can derive selection rules by attributing charge to the breaking parameter. Our normalization is such that this charge is $H(\lambda)$ $=-1$. While coefficients of order one are not determined in this framework, one can derive the parametric 
suppression of all couplings and consequently have an order-of-magnitude estimate of the physical parameters. Take, for example, the case in which the $H$ charges of the left-handed lepton doublets $L_{i}$ are positive. The parametric suppression of an entry in the neutrino mass matrix is determined by the charge that it carries, $\left(M_{\nu}\right)_{i j} \sim \lambda^{H\left(L_{i}\right)+H\left(L_{j}\right)+2 H(\phi)}$. The parametric suppression of the mixing angles and mass ratios can then be estimated (see, for example, Leurer et al., 1993),

$$
\begin{aligned}
& \left|U_{i j}\right| \sim \lambda^{H\left(L_{i}\right)-H\left(L_{j}\right),} \\
& m\left(\nu_{i}\right) / m\left(\nu_{j}\right) \sim \lambda^{2\left[H\left(L_{i}\right)-H\left(L_{j}\right)\right]} \quad(i \leqslant j) .
\end{aligned}
$$

If the various generations of left-handed fields (quarks or leptons) carry different $H$ charges, then, in general, the (quark or lepton) mixing angles are suppressed. For example, $\sin \theta_{C} \sim \lambda$ is naturally induced if the charges of the first two quark-doublet generations are chosen appropriately: $H\left(Q_{1}\right)-H\left(Q_{2}\right)=1$. A mixing angle of order one can be naturally induced if the charges of the corresponding lepton-doublet fields are equal to each other. Equation (164) shows, however, that in this class of models, independent of the charge assignments (as long as they are all positive), we have (Grossman and Nir, 1995)

$$
m\left(\nu_{i}\right) / m\left(\nu_{j}\right) \sim\left|U_{i j}\right|^{2} .
$$

Hence, for order-one mixing, there is no parametric suppression of the corresponding neutrino mass ratio and no hierarchy induced.

There is another possibility for inducing large lepton mixing which is unique to the case of Majorana neutrinos. Here one assigns, for example, opposite charges, $H\left(L_{i}\right)=-H\left(L_{j}\right)$, to the relevant lepton-doublet fields. The selection rules lead to the following structure of the mass matrix:

$$
M_{\nu} \sim \frac{v^{2}}{\Lambda_{\mathrm{NP}}}\left(\begin{array}{cc}
A \lambda^{2\left|H\left(L_{i}\right)\right|} & B \\
B & C \lambda^{2\left|H\left(L_{i}\right)\right|}
\end{array}\right), A, B, C=\mathcal{O}(1) .
$$

[In a supersymmetric framework, the combination of supersymmetry and the horizontal symmetry (Leurer et al., 1993, 1994) leads to a situation in which either $A$ or $C$ vanish.] This mass matrix has a pseudo-Dirac structure and it leads to near-maximal mixing and neardegenerate masses [see, for example, Joshipura and Rindani (2000b); Nir (2000)]:

$$
\sin ^{2} \theta=1 / 2-\mathcal{O}\left(\lambda^{2\left|H\left(L_{i}\right)\right|}\right), \quad \frac{\Delta m^{2}}{m^{2}}=\mathcal{O}\left(\lambda^{2\left|H\left(L_{i}\right)\right|}\right) .
$$

Of course, a pseudo-Dirac structure is inconsistent with mass hierarchy.

Here are a few mechanisms that have been suggested in the literature to induce strong hierarchy simultaneously with large mixing angle:

(i) Accidental hierarchy: the mass matrix (161) has $A, B, C=\mathcal{O}(1)$, and Eq. (163) is accidentally fulfilled. In the context of Abelian horizontal symmetries, this means that the mass ratio is numerically but not para- metrically suppressed (Binetruy et al., 1996; Elwood et al., 1998; Irges et al., 1998; Vissani, 1998, 2001b; Ellis et al., 1999; Sato and Yanagida, 2000). We should like to emphasize that, if the large-mixing-angle solution of the solar neutrino problem is correct, this is not an unlikely solution. While oscillation experiments give the ratio of masses-squared, the relevant quantity for theories is the ratio of masses. For the large-mixing-angle solution, $m_{2} / m_{3} \sim 0.1$, which is not a particularly small number and could easily arise from a combination of order-one terms.

(ii) Several sources for neutrino masses: the leading contribution to the neutrino mass matrix, which is responsible for the order-one mixing, is rank one. The lighter generation masses come from a different, subdominant source. This is, for example, the generic situation in supersymmetric models without $R$ parity (see, for example, Banks et al., 1995; Borzumati et al., 1996; Davidson and Losada, 2000). There, tree-level mixing with neutralinos can lead to large mixing, but gives a mass to only one neutrino generation, while the lighter masses arise at the loop level. Another realization of this principle would be the scenario of a seesaw mechanism with a single right-handed neutrino (Davidson and King, 1998; King, 1998, 1999, 2000; de Gouvea and Valle, 2001). For another example, within supersymmetric theories, see Borzumati and Nomura (2001).

(iii) Large mixing from the charged-lepton sector: it is possible that the neutrino mass matrix is hierarchical and nearly diagonal in the 2-3 sector, and the large mixing is coming from the diagonalization of the chargedlepton mass matrix. A variety of models have been constructed that give this structure of mass matrices, based on discrete horizontal symmetries (Grossman et al., 1998; Tanimoto, 1999b), holomorphic zeros (Grossman et al., 1998) and $\mathrm{U}(1)_{H}$ with two breaking parameters (Nir and Shadmi, 1999). For example, Grossman et al. (1998) work in the supersymmetric framework with a horizontal $\mathrm{U}(1) \times \mathrm{U}(1)$ symmetry. By an appropriate choice of horizontal charges, they obtain the following structure of lepton mass matrices (arbitrary coefficients of order one are omitted in the various entries):

$$
\begin{aligned}
& M_{\nu} \sim \frac{\left\langle\phi_{u}\right\rangle^{2}}{\Lambda_{\mathrm{NP}}}\left(\begin{array}{ccc}
\lambda^{2} & \lambda & \lambda \\
\lambda & 0 & 0 \\
\lambda & 0 & 1
\end{array}\right), \\
& M_{\ell^{ \pm}} \sim\left\langle\phi_{d}\right\rangle\left(\begin{array}{ccc}
\lambda^{8} & \lambda^{6} & \lambda^{4} \\
\lambda^{7} & \lambda^{5} & \lambda^{3} \\
\lambda^{7} & \lambda^{5} & \lambda^{3}
\end{array}\right),
\end{aligned}
$$

where the zero entries are a consequence of holomorphy. These matrices lead to

$$
\Delta m_{21}^{2} /\left|\Delta m_{32}^{2}\right| \sim \lambda^{3}, \quad\left|U_{\mu 3}\right| \sim 1,
$$

where the dominant contribution to the mixing angle comes from diagonalization of $M_{\ell^{ \pm}}$. The solar neutrino parameters of this model correspond to the largemixing-angle solution. 
(iv) Large mixing from the seesaw mechanism: it is possible that sterile neutrinos play a significant role in the flavor parameters of light, dominantly active neutrinos. In other words, an effective mass matrix of the form (161) with the condition (163) could be a result of selection rules applied in the full high-energy theory to a mass matrix that included both active and sterile neutrinos and the heavy, dominantly sterile ones integrated out (Altarelli and Feruglio, 1998; Barbieri et al., 1998; Eyal and Nir, 1999). It is interesting to note that in a large class of such models, the induced hierarchy would be too strong for the large-mixing-angle and smallmixing-angle solutions, unless at least three sterile neutrinos played a role in determining the low-energy parameters (Nir and Shadmi, 1999). An interesting example of a model of this type is presented by Altarelli and Feruglio (1998). They consider a horizontal U(1) symmetry broken by two parameters of opposite charges. By an appropriate choice of horizontal charges for the lepton fields, they obtain the following neutrino Dirac mass matrix $M_{D}$ and Majorana mass matrix for the sterile neutrinos $M_{N}$ :

$$
\begin{aligned}
& M_{D} \sim\left\langle\phi_{u}\right\rangle\left(\begin{array}{ccc}
\lambda^{3} & \lambda & \lambda^{2} \\
\lambda & \lambda^{\prime} & 1 \\
\lambda & \lambda^{\prime} & 1
\end{array}\right), \\
& M_{N} \sim \Lambda_{\mathrm{NP}}\left(\begin{array}{ccc}
\lambda^{2} & 1 & \lambda \\
1 & \lambda^{\prime 2} & \lambda^{\prime} \\
\lambda & \lambda^{\prime} & 1
\end{array}\right) .
\end{aligned}
$$

Integrating out of the three heavy neutrinos, the following Majorana mass matrix for the light neutrinos is obtained:

$$
\begin{aligned}
& M_{\nu} \sim \frac{\left\langle\phi_{u}\right\rangle^{2}}{\Lambda_{\mathrm{NP}}}\left(\begin{array}{ccc}
\lambda^{4} & \lambda^{2} & \lambda^{2} \\
\lambda^{2} & A & B \\
\lambda^{2} & B & C
\end{array}\right), \quad A, B, C=\mathcal{O}(1), \\
& \left|A C-B^{2}\right|=\mathcal{O}\left(\lambda \lambda^{\prime}\right) .
\end{aligned}
$$

If one were to apply the selection rules directly to $M_{\nu}$, the generic structure of Eq. (171) would be reproduced, but not the relation between the order-one coefficients in Eq. (172).

(v) A three-generation mechanism: approximate $L_{e}$ $-L_{\mu}-L_{\tau}$. One of the more interesting frameworks that produces all the observed features of neutrino flavor parameters is intrinsically a three-generation framework. One applies an approximate $L_{e}-L_{\mu}-L_{\tau}$ symmetry to the mass matrices of light, active neutrinos and of charged leptons. ${ }^{3}$ For the most general case, the symmetry is broken by small parameters, $\epsilon_{+}$and $\epsilon_{-}$, of charges +2 and -2 , respectively. The lepton mass matrices have the following form:

\footnotetext{
${ }^{3}$ Examples of this approach include Barbieri et al., 1998, 1999; Frampton and Glashow, 1999; Nir and Shadmi, 1999; Cheung and Kong, 2000; Joshipura and Rindani, 2000a; Mohapatra et al., 2000a; Nir, 2000; Shafi and Tavartkiladze, 2000d.
}

$$
\begin{aligned}
& M_{\nu} \sim \frac{\left\langle\phi_{u}\right\rangle^{2}}{\Lambda_{\mathrm{NP}}}\left(\begin{array}{ccc}
\epsilon_{-} & 1 & 1 \\
1 & \epsilon_{+} & \epsilon_{+} \\
1 & \epsilon_{+} & \epsilon_{+}
\end{array}\right), \\
& M_{\ell} \sim\left\langle\phi_{d}\right\rangle\left(\begin{array}{ccc}
\lambda_{e} & \lambda_{\mu} \epsilon_{-} & \lambda_{\tau} \epsilon_{-} \\
\lambda_{e} \epsilon_{+} & \lambda_{\mu} & \lambda_{\tau} \\
\lambda_{e} \epsilon_{+} & \lambda_{\mu} & \lambda_{\tau}
\end{array}\right),
\end{aligned}
$$

where the $\lambda_{i}$ 's allow for generic approximate symmetry that acts on the SU(2)-singlet charged leptons. The resulting neutrino masses are as follows:

$$
m_{1,2}=m\left\{1 \pm \mathcal{O}\left[\max \left(\boldsymbol{\epsilon}_{+}, \boldsymbol{\epsilon}_{-}\right)\right]\right\}, \quad m_{3}=m \mathcal{O}\left(\boldsymbol{\epsilon}_{+}\right) .
$$

Note that the quasidegenerate pair of mass eigenstates involved in the solar neutrino anomaly is heavier than the third, separated state. For the mixing angles, one finds

$$
\begin{aligned}
& \left|U_{\mu 3}\right|=\mathcal{O}(1), \quad U_{e 3}=\mathcal{O}\left[\max \left(\epsilon_{+}, \epsilon_{-}\right)\right], \\
& \left|U_{e 2}\right|^{2}=1 / 2-\mathcal{O}\left[\max \left(\epsilon_{+}, \epsilon_{-}\right)\right] .
\end{aligned}
$$

The overall picture is that, somewhat surprisingly, the lepton parameters (154)-(156) are not easy to account for with Abelian flavor symmetries. The simplest and most predictive models have difficulties in accommodating the large 2-3 mixing together with the strong 2-3 hierarchy. One can find more complicated models that naturally induce these parameters, but often at the cost of losing predictive power. In particular, it may be the case that, specifically for neutrinos, one cannot ignore the existence of heavy degrees of freedom (sterile neutrinos), well beyond the reach of direct experimental production, that affect the flavor parameters of the lowenergy effective theory. If true, this situation would mean that measuring the low-energy neutrino parameters cannot by itself make a convincing case for the idea of Abelian flavor symmetries. (An exception are models of approximate $L_{e}-L_{\mu}-L_{\tau}$ symmetry.)

Similar difficulties are encountered in the framework of non-Abelian symmetries. Again, the simplest models do not work and have to be extended in rather complicated ways (Barbieri, Hall, Kane, and Ross, 1999; Barbieri, Hall, and Strumia, 1999). In some cases, the nonAbelian symmetries can give testable (almost) exact relations between masses and mixing angles. For example, the model of Barbieri, Creminelli, and Romanino (1999) predicts $\sin \theta_{12}=\sqrt{m_{e} / m_{\mu}} \cos \theta_{23}$ and $\sin \theta_{13}=\sqrt{m_{e} / m_{\mu}} \sin \theta_{23}$. If it turns out that all three light neutrinos are quasidegenerate, non-Abelian symmetries will become an unavoidable ingredient in flavor model building, but the task of constructing realistic models will be very challenging. ${ }^{4}$ Radiative corrections

\footnotetext{
${ }^{4}$ See, for example, Fritzsch and Xing, 1996, 2000; Carone and Sher, 1998; Fukugita, Tanimoto, and Yanagida, 1998, 1999; Barbieri, Ross, and Strumia, 1999; Tanimoto, 1999a, 2000; Tanimoto, Watari, and Yanagida, 1999; Wetterich, 1999; Wu, 1999a, 1999b, 1999c; Perez, 2000; Ma and Rajasekaran, 2001.
} 
are an important issue when examining the naturalness of various models that account for quasidegeneracy among neutrinos (see, for example, Casas et al., 1999, 2000).

\section{IMPLICATIONS FOR MODELS OF NEW PHYSICS}

In this section we review the implications of neutrino physics for various extensions of the standard model. We do not attempt to describe all relevant models of new physics, but take two representative frameworks. We choose to focus on well-motivated models that were not constructed for the special purpose of explaining the neutrino parameters. Thus the neutrino parameters either test these frameworks or provide further guidance in distinguishing among different options for model building within each framework. The two frameworks are the following:

GUT's: here the overall scale of the neutrino masses and some features of the mixing provide interesting tests.

(ii) Large extra dimensions: in the absence of a high energy scale, the seesaw mechanism is not operative in this framework. The lightness of the neutrinos is a challenge.

\section{A. Grand unified theories}

There are two significant facts about the gauge symmetries of the standard model and the structure of its fermionic representations that motivate the idea of supersymmetric grand unification. First, GUT's provide a unification of the standard-model multiplets (for a recent review, see Wilczek, 2001). Second, in the framework of the supersymmetric standard model, the three gauge couplings unify (Dimopoulos, Raby, and Wilczek, 1981; Ibañez and Ross, 1981). The unification scale is given by (see, for example, Langacker and Polonsky, 1995)

$$
\Lambda_{\mathrm{GU}} \sim 3 \times 10^{16} \mathrm{GeV} .
$$

Further support for grand unification comes from the flavor sector: the masses of the bottom quark and the tau lepton are consistent with equal masses at $\Lambda_{\mathrm{GU}}$, as predicted by $\mathrm{SU}(5)$ and its extensions.

The evidence for neutrino masses from atmospheric neutrinos, the implied scale of $\Delta m_{\mathrm{atm}}^{2}$, and the required mixing of order one should be considered as three further triumphs of the grand unification idea.

First, as mentioned above, $\mathrm{SO}(10)$ theories and their extensions require that there exist singlet fermions. Neutrino masses are then a prediction of these theories.

Second, $\mathrm{SO}(10)$ theories naturally give the singlet fermions heavy masses at the $\mathrm{SO}(10)$ breaking scale and, furthermore, relate the neutrino Dirac mass matrix $M_{\nu}^{D}$ to the up-quark mass matrix $M_{u}$. Specifically, the naive $\mathrm{SO}(10)$ relation reads $M_{\nu}^{D}=M_{u}$. It follows that the mass of the heaviest among the three light neutrinos can be estimated:

$$
m_{3} \sim m_{t}^{2} / \Lambda_{\mathrm{SO}(10)} \sim 10^{-3} \mathrm{eV} .
$$

It requires only that the lightest of the three singlet fermion masses be a factor of 50 below the unification scale to induce neutrino masses at the scale appropriate for the atmospheric neutrino anomaly. In other words, the intriguing proximity of $\Lambda_{\mathrm{NP}}$ of Eq. (150) to $\Lambda_{\mathrm{GU}}$ of Eq. (176) finds a natural explanation in the framework of $\mathrm{SO}(10)$. In this sense the seesaw mechanism of GellMann, Ramond, and Slansky (1979) and of Yanagida (1979) predicted neutrino masses at a scale that is relevant to atmospheric neutrinos.

The third triumph, $\left|U_{\mu 3}\right|=\mathcal{O}(1)$, is more subtle but still quite impressive (Harvey, Reiss, and Ramond, 1982; Babu and Barr, 1996; Sato and Yanagida, 1998). Unlike the previous points, which were related to $\mathrm{SO}(10)$ GUT's, here the consistency relates to SU(5) GUT's. $\mathrm{SU}(5)$ theories relate the charged-lepton mass matrix $M_{\ell}$ to the down-quark mass matrix $M_{d}$. Specifically, the naive SU(5) relation reads $M_{\ell}=M_{d}^{T}$. It follows that

$$
\left|U_{\mu 3} V_{c b}\right| \sim m_{s} / m_{b} \text {. }
$$

Given the experimental values $\left|V_{c b}\right| \sim 0.04$ and $m_{s} / m_{b}$ $\sim 0.03$ we conclude that the naive $\mathrm{SU}(5)$ relations predict $\left|U_{\mu 3}\right| \sim 1$. Of course, $\left|U_{\mu 3}\right|$ is also affected by the diagonalization of the light-neutrino mass matrix, but there is no reason to assume that this contribution cancels out the charged-lepton sector in such a way that $\left|U_{\mu 3}\right| \ll 1$.

Many of the other naive $\mathrm{SO}(10)$ relations fail, as do many of the naive SU(5) relations. Specifically, $\mathrm{SO}(10)$ predicts vanishing CKM mixing and mass ratios such as $m_{c} / m_{t}=m_{s} / m_{b}$. SU(5) predicts $m_{s}=m_{\mu}$ and $m_{d}=m_{e}$. It is possible, however, that these bad predictions are corrected by subleading effects, while all the successful predictions (particularly $m_{b}=m_{\tau}, m_{\mu} / m_{\tau} \sim m_{s} / m_{b}$, and $\left.\left|U_{\mu 3} V_{c b}\right| \sim m_{s} / m_{b}\right)$ are retained since they depend on the leading contributions. This is demonstrated in a number of specific GUT models. ${ }^{5}$

The flavor structure of the first two neutrino generations depends on both the Majorana mass matrix for the singlet fermions and the subdominant effects that correct the flavor parameters of the first two generations of quarks and charged leptons. This part of GUT's is therefore much more model dependent. Explicit GUT models have been constructed that accommodate the various solutions of the solar neutrino problem. We shall not describe them in any detail here.

\section{B. Extra dimensions}

New ideas concerning the possibility of large additional dimensions and the world on a brane can lead to

\footnotetext{
${ }^{5}$ See, for example, Albright, Babu, and Barr, 1998; Albright and Barr, 1998, 1999a, 1999b, 2000a, 2000b, 2001; Altarelli and Feruglio, 1998, 1999a, 1999b; Berezhiani and Rossi, 1999, 2001; Hagiwara and Okamura, 1999; Shafi and Tavartkiladze, 1999, 2000a, 2000b, 2000c; Altarelli, Feruglio, and Masina, 2000a, 2000b; Babu, Pati, and, Wilczek, 2000; Maekawa, 2001.
} 
sources of neutrino masses that are very different from those we have discussed so far. For example, the small mass could be related to the large volume factor of the extra dimensions which suppresses the coupling to bulk fermions (Dienes et al., 1999; Mohapatra et al., 1999, 2000b; Lukas et al., 2000, 2001; Mohapatra and PerezLorenzana, 2000; Dienes and Sarcevic, 2001; ArkaniHamed et al., 2002), to the breaking of lepton number on a distant brane (Arkani-Hamed et al., 2002), or to the warp factor in the Randall-Sundrum framework (Grossman and Neubert, 2000). In this section, we briefly describe these three mechanisms.

The existence of large extra dimensions not only provides new ways of generating small neutrino masses but can also lead to interesting phenomenological consequences. In particular, the phenomenology of matter oscillations in the Sun can be affected (Dvali and Smirnov, 1999; Barbieri et al., 2000; Lukas et al., 2000; Ma et al., 2000; Caldwell et al., 2001a, 2001b). Other phenomenological implications can be used to constrain the parameters of the extra dimensions (Faraggi and Pospelov, 1999; Das and Kong, 1999; Ioannisian and Pilaftsis, 2000).

\section{Coupling to bulk fermions}

It is possible that we live on a brane with three spatial dimensions that is embedded in a spacetime with $n$ additional large spatial dimensions (Arkani-Hamed et al., 1998). This idea has the potential of providing an understanding of the hierarchy between the gravitational mass scale $M_{\mathrm{Pl}}$ and the electroweak scale $m_{Z}$. The hope is to solve the hierarchy problem by avoiding a fundamental high energy (that is Planck or GUT) scale altogether. The observed Planck scale, $M_{\mathrm{Pl}}=\left(G_{N}\right)^{-1 / 2} \sim 10^{19} \mathrm{GeV}$, is related to the fundamental Planck scale (most likely the string scale) of the $4+n$ dimensional theory, $M_{*}$, by the Gauss law,

$$
M_{\mathrm{Pl}}^{2}=M_{*}^{n+2} V_{n},
$$

where $V_{n}$ is the volume of the $n$-dimensional extra space. This picture has dramatic phenomenological consequences for particle physics and cosmology. Such a situation also poses an obvious problem for neutrino physics. If there is no scale of physics as high as Eq. (151), the seesaw mechanism for suppressing light neutrino masses cannot be implemented. Conversely, if there are singlet neutrinos that are confined to the threedimensional brane where the active neutrinos live, one expects their mass to be at the string scale, $M_{*}$, which in these models is much smaller than the four-dimensional $M_{\mathrm{Pl}}$, perhaps as small as a few $\mathrm{TeV}$, and the resulting light-neutrino masses are well above the scales of atmospheric or solar neutrinos.

Within this framework the first implication of the evidence for neutrino masses is that there had better be no singlet fermions confined to the brane. Alternatively, the model must include some special ingredients to avoid ordinary seesaw masses.

On the other hand, it is typical in this framework that there be singlet fermions in the bulk. This would be the case, for example, with modulinos, the Fermionic partners of the moduli fields that are generic to string theories. The crucial point is that the Yukawa interaction between bulk singlet fermions and brane active neutrinos is highly suppressed by a large volume factor of the $n$ extra dimensions, $1 / \sqrt{V_{n}}$. This suppression factor reflects the small overlap between the wave functions of the sterile neutrino in the bulk and the active one on the brane. By construction, this factor provides a suppression of the neutrino Dirac mass by the ratio $v / M_{\mathrm{Pl}}$. Consequently, large extra dimensions provide a natural source of very small Dirac mass terms for the active neutrinos.

The consequences for neutrino masses and mixing depend on the details of the physics of the bulk neutrinos. The possible scenarios are clearly described by Lukas et al. (2000). There an explicit derivation of the effective four-dimensional action from a five-dimensional one is given. The final result for the Dirac mass is as follows:

$$
m_{i}^{\text {Dir }}=\frac{v Y_{i}}{\sqrt{V_{n} M_{*}^{n}}}=Y_{i} v \frac{M_{*}}{M_{\mathrm{Pl}}} .
$$

Here $Y_{i}$ is the Yukawa coupling between the lepton doublet $L_{i}$ and a bulk singlet, $M_{*}$ is the string scale, and $V_{n}$ is the volume of the $n$-dimensional extra space. The usual Dirac mass is of order $Y v$, but in Eq. (180) we see explicitly the $\left(M_{*} / M_{\mathrm{Pl}}\right)$ suppression factor in the effective four-dimensional Yukawa couplings, leading to highly suppressed Dirac mass terms.

Being a bulk state, the singlet fermion has a whole tower of Kaluza-Klein associated states which are all coupled to the left-handed brane neutrinos. In the simplest scenario and for one extra dimension of radius $R$, the masses of all Kaluza-Klein states are determined by the scale $1 / R$ :

$$
m_{n}^{2}=\frac{n^{2}}{R^{2}}
$$

In more general scenarios there can be other bulk mass terms and the masses Kaluza-Klein states receive additional contributions. Then the lightest Kaluza-Klein mass can be taken as an independent parameter, while the mass splitting between the states is still determined by the scale $1 / R$.

Let us denote by $M_{\min }$ the lightest mass in the KaluzaKlein spectrum and by $\Delta m$ the mass scale that is relevant to atmospheric or to solar neutrino oscillations. Then one can distinguish three cases:

(a) $1 / R \gg \Delta m$ and $M_{\min } \gg \Delta m$. The Kaluza-Klein states play no direct role in these oscillations. Their main effect is to give seesaw masses to the active brane neutrinos.

(b) $1 / R \gg \Delta m$ and $M_{\min } \lesssim \Delta m$. The situation is equivalent to conventional models containing a small number of sterile states. In particular, for $M_{\min }=0$ it is a possible framework for light Dirac neutrinos. For small but nonvanishing $M_{\min }$ it may lead to the 
four-neutrino mixing scenarios. However these scenarios are not particularly favored.

(c) $1 / R \leq \Delta m$ and $M_{\min } \leq \Delta m$. A large number of bulk modes can take direct part in the oscillation phenomena. This situation modifies in a very interesting way the solution to the solar neutrino problem that involves MSW resonance conversion into a sterile neutrino. Now the $\nu_{e}$ can oscillate into a whole set of Kaluza-Klein states. However, as discussed in Sec. IV, solar oscillations into sterile states are now strongly disfavored by the comparison of the charged-current and neutral-current fluxes at SNO.

In summary, large extra dimensions with sterile bulk fermions provide a natural explanation for light neutrino masses. In the simplest realization, in which there are no bulk mass terms and lepton number conservation is imposed in the bulk-brane couplings, light Dirac masses are generated. In the general case, the light neutrinos can be either Dirac or Majorana particles, depending on the details of the physics of the bulk neutrinos.

\section{Lepton number breaking on a distant brane}

In its simplest realization, the mechanism described above provides a natural way of generating light Dirac masses for neutrinos. In the framework of extra dimensions one could also generate small Majorana masses by an alternative mechanism in which lepton number is broken on a distant brane (Arkani-Hamed et al., 2002). Imagine that lepton number is spontaneously broken at the scale $M_{*}$ on a different brane located a distance $r$ from our brane. Further assume that the information about this breaking is communicated to our brane by a bulk field with a mass $m$. For the sake of concreteness we take the case that $m r \gg 1$ and there are two extra dimensions. The resulting Majorana mass for the active neutrinos can be estimated as follows:

$$
m_{\nu} \sim \frac{v^{2}}{M_{*}} e^{-m r}
$$

We learn that the naive seesaw mass, of order $v^{2} / M_{*}$, is suppressed by a small exponential factor. The consequences for the neutrino spectrum depend on various model-dependent features: the number of large extra dimensions, the string scale, the distance between our brane and the brane where lepton number is broken, and the mass of the mediating bulk field.

A variant of the above two mechanisms can be implemented if the standard-model fields are confined to a thick wall (Arkani-Hamed and Schmaltz, 2000). Dirac masses are suppressed if left-handed and right-handed neutrinos are located at different points in the wall and consequently there is an exponentially small overlap of their wave functions. Majorana masses are suppressed if lepton number is spontaneously broken by a vacuum expectation value that is localized within the wall but at some distance from the lepton doublet fields.

\section{The warp factor in the Randall-Sundrum scenario}

A different setup for the extra dimensions (leading to a different explanation of the $v / M_{\mathrm{Pl}}$ hierarchy) was proposed by Randall and Sundrum (1999). They considered one extra dimension parametrized by a coordinate $y$ $=r_{c} \phi$, where $-\pi \leqslant \phi \leqslant+\pi . r_{c}$ is the radius of the compact dimension, and the points $(x, \phi)$ and $(x,-\phi)$ are identified. A visible brane is located at $\phi=\pi$ and a hidden brane at $\phi=0$. This setup leads to the following nonfactorizable metric:

$$
d s^{2}=e^{-2 k r_{c}|\phi|} \eta_{\mu \nu} d x^{\mu} d x^{\nu}-r_{c}^{2} d \phi^{2} .
$$

The parameter $k$ is the bulk curvature. All dimensionful parameters in the effective theory on the visible brane are suppressed by the warp factor, $\epsilon \equiv e^{-k r_{c} \pi}$. With $k r_{c}$ $\approx 12$, that is, $\epsilon \sim 10^{-16}$, this mechanism produces physical masses of order $v$ from fundamental masses of order $M_{\mathrm{Pl}}$.

The two mechanisms described above to generate small neutrino masses do not work in this scenario because the extra dimensions are small and there is no volume suppression factor available. Grossman and Neubert (2000) proposed a different mechanism for generating small masses in the Randall-Sundrum framework. (For a different mechanism, see Huber and Shafi, 2001.) With appropriate choice of orbifold boundary conditions, it is possible to locate the zero mode of a right-handed bulk neutrino on the hidden brane. If the fundamental mass scale $m$ of the bulk fermions is larger than half the curvature $k$ of the compact dimension, the wave function of the right-handed zero mode on the visible brane is power-suppressed in the ratio $v / M_{\mathrm{Pl}}$. Coupling the bulk fermions to the Higgs and lepton doublet fields yields

$$
m_{\nu} \sim v\left(\frac{v}{M_{\mathrm{Pl}}}\right)^{m / k-1 / 2}
$$

Note that the relation between the neutrino mass and the weak scale is generically different from the seesaw mechanism (except for the special case $m / k=3 / 2$ ).

To summarize, in the presence of large extra dimensions, neutrino masses could be suppressed by the large volume factor if the left-handed neutrinos couple to the bulk fermions (being Dirac for massless bulk fermions), or by the distance to a brane where lepton number is broken (which generate Majorana masses). In the Randall-Sundrum framework, the suppression can be induced by a power of the warp factor. The detailed structure of a neutrino mass hierarchy and mixing is often related to the parameters that describe the extra dimensions.

\section{CONCLUSIONS}

Strong evidence for neutrino masses and mixing has been coming from various neutrino oscillation experiments in recent years. First, atmospheric neutrinos show a deviation from the expected ratio between the $\nu_{\mu}$ and $\nu_{e}$ fluxes. Furthermore, the $\nu_{\mu}$ flux has strong zenith- 
angle dependence. The simplest interpretation of these results is that there are $\nu_{\mu}-\nu_{\tau}$ oscillations with the following mass and mixing parameters:

$$
\begin{aligned}
& 1.4 \times 10^{-3}<\Delta m_{\mathrm{atm}}^{2}<6 \times 10^{-3} \mathrm{eV}^{2}, \\
& 0.4<\tan ^{2} \theta_{\mathrm{atm}}<3.0 .
\end{aligned}
$$

The ranges quoted here correspond to the results of the global three-neutrino analysis presented in Sec. VII [Eq. (147)]. Second, the total rates of solar neutrino fluxes are smaller than the theoretical expectations. The suppression is different in the various experiments (which are sensitive to different energy ranges). The recently announced measurements of neutral-current and charged-current fluxes at SNO provide a $5.3 \sigma$ signal for neutrino flavor transition that is independent of the solar model. The simplest interpretation of these results is that there are $\nu_{e}-\nu_{a}$ oscillations (where $\nu_{a}$ is some combination of $\nu_{\mu}$ and $\nu_{\tau}$ ) with the following set of mass and mixing parameters:

$$
\begin{aligned}
& 2.4 \times 10^{-5}<\Delta m_{\odot}^{2}<2.4 \times 10^{-4} \mathrm{eV}^{2}, \\
& 0.27<\tan ^{2} \theta_{\odot}<0.77 \text { (large mixing angle), }
\end{aligned}
$$

where again the ranges quoted correspond to the results of the global analysis given in Eq. (147). From the global analysis, an upper bound on a third mixing angle arises, driven mainly by the negative results of the reactor experiments in combination with the deduced $\Delta m_{\mathrm{atm}}^{2}$ from the atmospheric neutrino data:

$$
\sin \theta_{\text {reactor }} \leqslant 0.24 \text {. }
$$

The smallness of this angle guarantees that the results of the three-neutrino analysis combining the atmospheric and solar neutrino data will be close to the two separate two-neutrino analyses.

The evidence for neutrino masses implies that the standard model cannot be a complete picture of Nature. In particular, if the standard model is only a low-energy effective theory, very light neutrino masses are expected. The scale at which the standard-model picture is no longer valid is inversely proportional to the scale of neutrino masses. Specifically,

$$
m_{\nu} \geq \sqrt{\Delta m_{\mathrm{atm}}^{2}} \sim 0.05 \mathrm{eV} \Rightarrow \Lambda_{\mathrm{NP}} \lesssim 10^{15} \mathrm{GeV} .
$$

We learn that there is a scale of new physics well below the Planck scale.

The scale of new physics in Eq. (188) is intriguingly close to the scale of coupling unification. Indeed, since GUT's with an $\mathrm{SO}(10)$ (or larger) gauge group predict neutrino masses, plausibly at the $0.1 \mathrm{eV}$ scale, the atmospheric neutrino data can be taken as additional support for the GUT idea. The large mixing angle in Eq. (185) also finds a natural and quite generic place in GUT's.

The measured values of the neutrino flavor parameters are useful in testing various ideas to explain the flavor puzzle. Quite a few of the simplest models that explain the smallness and hierarchy in the quark-sector parameters fail to explain the neutrino parameters. The neutrino parameters have some features that are quite unique. In particular, the two mixing angles $\theta_{\mathrm{atm}}$ and $\theta_{\odot}$ are large. As concerns the mass hierarchy, $\Delta m_{\odot}^{2} / \Delta m_{\text {atm }}^{2}$, the situation is still ambiguous. If there is strong hierarchy, it is not easy to accommodate it together with large mixing angles. If the hierarchy is mild enough to be consistent with just accidental suppression, and if, in addition, the third mixing angle (bounded by reactor experiments) is not very small, it could well be that there is no hierarchy in the neutrino parameters at all. That would call for flavor frameworks that gave a different structure for charged and neutral fermion parameters. Other possibilities, such as quasidegeneracy in masses or bimaximal mixing, also call for special structure in the neutrino sector that is very different from that of the quark sector.

The mass scales involved in Eqs. (185) and (186) have implications for many other frameworks of new physics. In particular, they can help discriminate between various options in the framework of models with extra dimensions, models of supersymmetry without $R$ parity, leftright symmetric models, etc.

Another hint for neutrino masses comes from the LSND experiment. Here, however, the signal is presently observed by a single experiment, and further experimental testing is required. The simplest interpretation of the LSND data is that there are $\nu_{e} \rightarrow \nu_{\mu}$ oscillations with $\Delta m_{\mathrm{LSND}}^{2}=\mathcal{O}\left(1 \mathrm{eV}^{2}\right)$ and $\sin ^{2} 2 \theta_{\mathrm{LSND}}$ $=\mathcal{O}(0.003)$. The fact that $\Delta m_{\mathrm{LSND}}^{2} \gg \Delta m_{\mathrm{atm}}^{2}, \Delta m_{\odot}^{2}$ means that the three results cannot be explained by oscillations among the three active neutrinos alone.

If the LSND result is confirmed, then more dramatic modifications of the standard model will be required. The simplest extension, that is, the addition of a light singlet neutrino, is not excluded but it does run into difficulties related to the fact that oscillations into purely sterile neutrinos fit neither the atmospheric nor the solar neutrino data.

The good news is that there has been a lot of progress in neutrino physics in recent years. Measurements of both atmospheric and solar neutrino fluxes make the case for neutrino masses and mixing more and more convincing. Many theoretical ideas are being excluded, while others have at last experimental guidance in their construction. The other piece of good news is that there is a lot of additional experimental information concerning neutrino physics to come in the near future. We are guaranteed to learn much more.

\section{Note added}

In December 2002, the KamLAND Collaboration released their first data on the measurement of the flux of $\bar{\nu}_{e}$ 's from distant nuclear reactors (Eguchi et al., 2002). Below we briefly present the main implications of this result.

\section{KamLAND results}

KamLAND have measured the flux of $\bar{\nu}_{e}$ 's from distant nuclear reactors. In an exposure of 162 ton yr (145.1 
days), the ratio of the number of observed inverse $\beta$-decay events to the number of events expected without oscillations is

$$
R_{\text {KamLAND }}=0.611 \pm 0.094 \text {, }
$$

for $E_{\bar{\nu}_{e}}>3.4 \mathrm{MeV}$. This deficit is inconsistent with the expected rate for massless $\bar{\nu}_{e}$ 's at the $99.95 \%$ confidence level.

KamLAND have also presented the energy dependence of these events in the form of the prompt energy $\left(E_{\text {prompt }} \simeq E_{\bar{v}_{e}}+m_{p}-m_{n}\right)$ spectrum with 17 bins of width $0.425 \mathrm{MeV}$ for $E_{\text {prompt }}>0.9 \mathrm{MeV}$. To eliminate the background from geo-neutrinos, only bins with $E_{\text {prompt }}$ $>2.6 \mathrm{MeV}$ are used. The measured spectrum shows a clear deficit, but there is no significant signal of any energy dependence of this effect.

The KamLAND results can be interpreted in terms of $\bar{\nu}_{e}$ oscillations with parameters shown in Fig. 37(a) (from Bahcall, Gonzalez-Garcia, and Peña-Garay, 2002d). Since KamLAND is a disappearance experiment and, furthermore, matter effects are small, the experiment is not sensitive to the flavor into which $\bar{\nu}_{e}$ oscillates. The allowed region has three local minima and it is separated into "islands." These islands correspond to oscillations with wavelengths that are approximately tuned to the average distance between the reactors and the detector, $180 \mathrm{~km}$. The local minimum in the lowest-mass island $\left(\Delta m^{2}=1.5 \times 10^{-5} \mathrm{eV}^{2}\right)$ corresponds to an approximate first maximum in the oscillation probability (minimum in the event rate). The overall best-fit point $\left(\Delta m^{2}=7.1\right.$ $\times 10^{-5} \mathrm{eV}^{2}$ and $\tan ^{2} \theta=0.52,1.92$ ) corresponds approximately to the second maximum in the oscillation probability. For the same $\Delta m^{2}$, maximal mixing is only slightly less favored, $\Delta \chi^{2}=0.4$. Thus with the present statistical accuracy, KamLAND cannot discriminate between a large-but-not-maximal and maximal mixing.

Because there is no significant evidence of energy distortion, the allowed regions for the KamLAND analysis extend to high $\Delta m^{2}$ values for which oscillations would be averaged and the event reduction would be energy independent. These solutions, however, are ruled out by the nonobservation of a deficit at shorter baselines, and in particular by the negative results of $\mathrm{CHOOZ}$, which rule out solutions with $\Delta m^{2} \gtrsim 1(0.8) \times 10^{-3} \mathrm{eV}^{2}$ at $3 \sigma$ (99\% C.L.).

\section{Consequences for solar neutrinos}

The most important aspect of Fig. 37(a) is the demonstration by the KamLAND results that antineutrinos oscillate with parameters that are consistent with the large-mixing-angle solar neutrino solution. Under the assumption that $C P T$ is satisfied, the antineutrino measurements by KamLAND apply directly to the neutrino sector, and the two sets of data can be combined to obtain the globally allowed oscillation parameters (in fact, the KamLAND results already show that $C P T$ is satisfied in the neutrino sector to a good approximation).
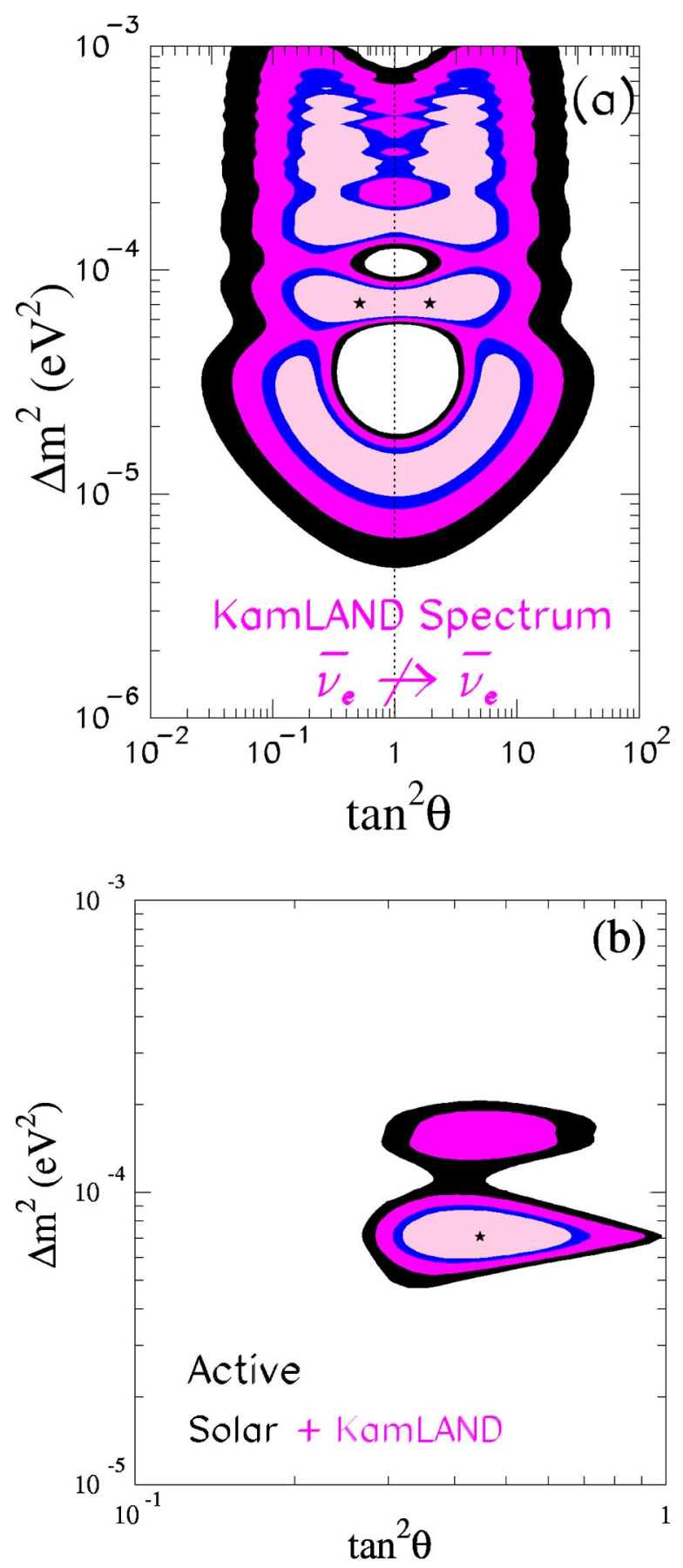

FIG. 37. The impact of KamLAND results on the allowed regions (at 90\%, 95\%, 99\%, and 99.7\% C.L.) in the $\left(\Delta m_{21}^{2}, \tan ^{2} \theta_{12}\right)$ plane. (a) A combined analysis of KamLAND and $\mathrm{CHOOZ}$ results. (b) A combined analysis of KamLAND and solar data (Color in online edition).

The results of such an analysis are shown in Fig. 37(b) (from Bahcall, Gonzalez-Garcia, and Peña-Garay, 2002d).

Comparing Fig. 37(a) with Fig. 37(b), we can see that the impact of the KamLAND results is to narrow down the allowed parameter space in a very significant way. In particular, large-mixing-angle region is the only remaining allowed solution. The low-mass solution is excluded at $4.8 \sigma$ and vacuum solutions are excluded at $4.9 \sigma$. The 
once-favored small-mixing-angle solution is now excluded at $6.1 \sigma$. Within the low-mixing-angle region, the main effect of KamLAND is the reduction in the allowed range of $\Delta m^{2}$, while the impact on the determination of the mixing angle is marginal.

Furthermore, various alternative solutions to the solar neutrino problem which predict that there should be no deficit in the KamLAND experiment (such as neutrino decay or new flavor-changing interactions) are now excluded at $3.6 \sigma$.

\section{Effect on three-neutrino mixing scenarios}

The $\bar{\nu}_{e}$ survival probability at KamLAND in the three-neutrino framework is

$$
\begin{aligned}
P\left(\bar{\nu}_{e} \leftrightarrow \bar{\nu}_{e}\right)= & \sin ^{4} \theta_{13}+\cos ^{4} \theta_{14}\left[1-\sum_{i} f_{i} \sin ^{2} 2 \theta_{12}\right. \\
& \left.\times \sin ^{2}\left(\frac{1.27 \Delta m_{21}^{2}\left[\mathrm{eV}^{2}\right] L_{i}[\mathrm{~km}]}{E_{\nu}[\mathrm{GeV}]}\right)\right],
\end{aligned}
$$

where $L_{i}$ is the distance of reactor $i$ to KamLAND, and $f_{i}$ is the fraction of the total neutrino flux that comes from reactor $i$. Equation (190) is obtained with two approximations: (a) matter effects are neglected at KamLAND-like baselines, and (b) for $\Delta m^{2}$ $\geq 10^{-4} \mathrm{eV}^{2}$, the KamLAND energy resolution is not good enough to resolve the corresponding oscillation wavelength, so the oscillation phase associated to $\Delta m_{31}^{2}$ is averaged to $\frac{1}{2}$.

Equation (190) shows that a small $\theta_{13}$ does not significantly affect the shape of the measured spectrum. On the other hand, the overall normalization is scaled by $\cos ^{4} \theta_{13}$ and this factor may introduce a non-negligible effect. Within its present accuracy, however, the KamLAND experiment cannot provide any further significant constraint on $\tan ^{2} \theta_{13}$ and the range in Eq. (190) still holds (see also Fogli et al., 2002).

Thus the main effect of the KamLAND results on the global three-neutrino analysis of solar, atmospheric, and reactor data is to modify the allowed range of $\Delta m_{21}^{2}$ in Eq. (190) to

$$
5.5 \times 10^{-5}<\Delta m_{21}^{2} / \mathrm{eV}^{2}<1.9 \times 10^{-4} \text {. }
$$

The allowed range of $\theta_{12}$ and the corresponding entries of the mixing matrix $U$ are only marginally affected by the inclusion of the KamLAND data.

\section{ACKNOWLEDGMENTS}

We thank M. Maltoni and C. Peña-Garay for providing us with updates of our previous published results. We thank Michael Dine and Yuval Grossman for critical reading of the manuscript. M.C.G.-G. thanks the Weizmann Institute, where this review was finalized, for their warm hospitality and the Albert Einstein Minerva Center for Theoretical Physics for financial support. M.C.G.-G. was supported by the European Union HPMF-CT-2000-00516. This work was also supported by the Spanish DGICYT under Grants Nos. PB98-0693 and FPA2001-3031, and by the ESF network 86. Y.N. was supported by the Israel Science Foundation founded by the Israel Academy of Sciences and Humanities.

\section{REFERENCES}

Abdurashitov, J. N., et al. (SAGE Collaboration), 1999, Phys. Rev. C 60, 055801.

Abdurashitov, J. N., et al. (SAGE Collaboration), 2002, J. Exp. Theor. Phys. 95, 181.

Ables, E., et al. (MINOS Collaboration), 1995, FERMILABPROPOSAL-P-875.

Achar, H., et al., 1965, Phys. Lett. 18, 196.

Achkar, B., et al. (Bugey Collaboration), 1995, Nucl. Phys. B 434, 503.

Aglietta, M., et al. (Nusex Collaboration), 1989, Europhys. Lett. 8, 611.

Agrawal, V., T. Gaisser, P. Lipari, and T. Stanev, 1996, Phys. Rev. D 53, 1314.

Aguilar, A., et al. (LSND Collaboration), 2001, Phys. Rev. D 64, 112007.

Ahmad, Q. R., et al. (SNO Collaboration), 2001, Phys. Rev. Lett. 87, 071301.

Ahmad, Q. R., et al. (SNO Collaboration), 2002a, Phys. Rev. Lett. 89, 011301.

Ahmad, Q. R., et al. (SNO Collaboration), 2002b, Phys. Rev. Lett. 89, 011302.

Ahn, S. H., et al. (K2K Collaboration), 2001, Phys. Lett. B 511, 178.

Ahrens, L. A., et al. (BNL E734 Collaboration), 1987, Phys. Rev. D 36, 702.

Akhmedov, E. K., 1988, Yad. Fiz. 47, 475 [Sov. J. Nucl. Phys. 47, 301 (1988)].

Akhmedov, E. K., 1999, Nucl. Phys. B 538, 25.

Akhmedov, E. K., A. Dighe, P. Lipari, and A. Yu. Smirnov, 1999, Nucl. Phys. B 542, 3.

Albright, C. H., K. S. Babu, and S. M. Barr, 1998, Phys. Rev. Lett. 81, 1167.

Albright, C. H., and S. M. Barr, 1998, Phys. Rev. D 58, 013002. Albright, C. H., and S. M. Barr, 1999a, Phys. Lett. B 452, 287. Albright, C. H., and S. M. Barr, 1999b, Phys. Lett. B 461, 218. Albright, C. H., and S. M. Barr, 2000a, Phys. Rev. D 62, 093008.

Albright, C. H., and S. M. Barr, 2000b, Phys. Rev. Lett. 85, 244. Albright, C. H., and S. M. Barr, 2001, Phys. Rev. D 64, 073010. Allison, W. W. M., et al. (Soudan Collaboration), 1999, Phys. Lett. B 449, 137.

Altarelli, G., and F. Feruglio, 1998, J. High Energy Phys. 9811, 021.

Altarelli, G., and F. Feruglio, 1999a, Phys. Lett. B 451, 388. Altarelli, G., and F. Feruglio, 1999b, Phys. Rep. 320, 295.

Altarelli, G., F. Feruglio, and I. Masina, 2000a, Phys. Lett. B 472, 382.

Altarelli, G., F. Feruglio, and I. Masina, 2000b, J. High Energy Phys. 0011, 040.

Ambrosio, M., et al. (MACRO Collaboration), 2001, Phys. Lett. B 517, 59.

Apollonio, M., et al. (CHOOZ Collaboration), 1999, Phys. Lett. B 466, 415.

Arkani-Hamed, N., S. Dimopoulos, and G. Dvali, 1998, Phys. Lett. B 429, 263. 
Arkani-Hamed, N., S. Dimopoulos, G. Dvali, and J. MarchRussell, 2002, Phys. Rev. D 65, 024032.

Arkani-Hamed, N., and M. Schmaltz, 2000, Phys. Rev. D 61, 033005.

Arpesella, C., C. Broggini, and C. Cattadori, 1996, Astropart. Phys. 4, 333.

Astier, P., et al. (NOMAD Collaboration), 2001, Nucl. Phys. B 611, 3 .

Athanassopoulos, C., et al., 1995, Phys. Rev. Lett. 75, 2650.

Athanassopoulos, C., et al., 1996, Phys. Rev. Lett. 77, 3082.

Athanassopoulos, C., et al., 1998, Phys. Rev. Lett. 81, 1774.

Ayres, D. S., B. Cortez, T. K. Gaisser, A. K. Mann, R. E. Shrock, and L. R. Sulak, 1984, Phys. Rev. D 29, 902.

Babu, K. S., and S. M. Barr, 1996, Phys. Lett. B 381, 202.

Babu, K. S., J. C. Pati, and F. Wilczek, 2000, Nucl. Phys. B 566, 33.

Bahcall, J. N., 1989, Neutrino Astrophysics (Cambridge University Press, Cambridge).

Bahcall, J. N., 1997, Phys. Rev. C 56, 3391.

Bahcall, J. N., 2002a, Phys. Rev. C 65, 015802.

Bahcall, J. N., 2002b, Phys. Rev. C 65, 025801.

Bahcall, J. N., N. A. Bahcall, and G. Shaviv, 1968, Phys. Rev. Lett. 20, 1209.

Bahcall, J. N., S. Basu, and H. M. Pinsonneault, 1998, Phys. Lett. B 433, 1.

Bahcall, J. N., and H. A. Bethe, 1990, Phys. Rev. Lett. 65, 2233.

Bahcall, J. N., and R. Davis, 1976, Science 191, 264.

Bahcall, J. N., M. C. Gonzalez-Garcia, and C. Peña-Garay, 2001, J. High Energy Phys. 0108, 014.

Bahcall, J. N., M. C. Gonzalez-Garcia, and C. Peña-Garay, 2002a, J. High Energy Phys. 0204, 007.

Bahcall, J. N., M. C. Gonzalez-Garcia, and C. Peña-Garay, 2002b, Phys. Rev. C 66, 035802.

Bahcall, J. N., M. C. Gonzalez-Garcia, and C. Peña-Garay, 2002c, J. High Energy Phys. 0207, 054.

Bahcall, J. N., M. C. Gonzalez-Garcia, and C. Peña-Garay, 2002d, J. High Energy Phys. 0302, 009.

Bahcall, J. N., M. Kamionkowsky, and A. Sirlin, 1995, Phys. Rev. D 51, 6146.

Bahcall, J. N., P. I. Krastev, and E. Lisi, 1997, Phys. Rev. C 55, 494.

Bahcall, J. N., P. I. Krastev, and A. Yu. Smirnov, 1998, Phys. Rev. D 58, 096016.

Bahcall, J. N., P. I. Krastev, and A. Yu. Smirnov, 2000, Phys. Rev. D 62, 093004.

Bahcall, J. N., and M. H. Pinsonneault, 1992, Rev. Mod. Phys. 64, 885.

Bahcall, J. N., and H. M. Pinsonneault, 1995, Rev. Mod. Phys. 67, 781.

Bahcall, J. N., H. M. Pinsonneault, and S. Basu, 2001, Astrophys. J. 555, 990.

Bahcall, J. N., and R. K. Ulrich, 1988, Rev. Mod. Phys. 60, 297. Baltz, A. J., and J. Weneser, 1987, Phys. Rev. D 35, 528.

Baltz, A. J., and J. Weneser, 1988, Phys. Rev. D 37, 3364.

Banks, T., Y. Grossman, E. Nardi, and Y. Nir, 1995, Phys. Rev. D 52, 5319.

Barbieri, R., P. Creminelli, and A. Romanino, 1999, Nucl. Phys. B 559, 17.

Barbieri, R., P. Creminelli, and A. Strumia, 2000, Nucl. Phys. B $\mathbf{5 8 5}, 28$.

Barbieri, R., L. J. Hall, G. L. Kane, and G. G. Ross, 1999, e-print hep-ph/9901228.
Barbieri, R., L. J. Hall, D. Smith, A. Strumia, and N. Weiner, 1998, J. High Energy Phys. 9812, 017.

Barbieri, R., L. J. Hall, and A. Strumia, 1999, Phys. Lett. B 445, 407.

Barbieri, R., G. G. Ross, and A. Strumia, 1999, J. High Energy Phys. 9910, 020.

Barenboim, G., L. Borissov, J. Lykken, and A. Yu. Smirnov, 2001, J. High Energy Phys. 0210, 001.

Barger, V., D. Marfatia, and K. Whisnant, 2002, Phys. Rev. Lett. 88, 011302.

Barger, V., D. Marfatia, K. Whisnant, and B. P. Wood, 2002, Phys. Lett. B 537, 179.

Barger, V., S. Pakvasa, T. J. Weiler, and K. Whisnant, 1998a, Phys. Rev. D 58, 093016.

Barger, V., S. Pakvasa, T. J. Weiler, and K. Whisnant, 1998b, Phys. Lett. B 437, 107.

Barger, V., and K. Whisnant, 1988, Phys. Lett. B 209, 365.

Barger, V., and K. Whisnant, 1999, Phys. Rev. D 59, 093007.

Barger, V., K. Whisnant, and R. J. N. Phillips, 1980, Phys. Rev. D 22, 1636.

Barr, G., T. K. Gaisser, and T. Stanev, 1989, Phys. Rev. D 39, 3532.

Battistoni, G., 2001, Nucl. Phys. B, Proc. Suppl. 100, 101.

Battistoni, G., A. Ferrari, P. Lipari, T. Montaruli, P. R. Sala, and T. Rancati, 2000, Astropart. Phys. 12, 315.

Bazarko, A., et al. (MiniBooNE Collaboration), 2000, Nucl. Phys. B, Proc. Suppl. 91, 210.

Becker-Szendy, R., et al. (IMB Collaboration), 1992, Phys. Rev. D 46, 3720.

Bemporad, C., G. Gratta, and P. Vogel, 2002, Rev. Mod. Phys. 74, 297.

Berezhiani, Z., and A. Rossi, 1999, J. High Energy Phys. 9903, 002.

Berezhiani, Z., and A. Rossi, 2001, Nucl. Phys. B 594, 113.

Berezinsky, V., 2001, Astropart. Phys. 17, 509.

Berger, M. S., and K. Siyeon, 2001, Phys. Rev. D 63, 057302.

Bergmann, S., Y. Grossman, and E. Nardi, 1999, Phys. Rev. D 60, 093008.

Bilenky, S. M., J. Hosek, and S. T. Petcov, 1980, Phys. Lett. B 94, 495.

Bilenky, S. M., D. Nicolo, and S. T. Petcov, 2001, Phys. Lett. B 538, 77.

Bilenky, S. M., S. Pascoli, and S. T. Petcov, 2001a, Phys. Rev. D 64, 053010 .

Bilenky, S. M., S. Pascoli, and S. T. Petcov, 2001b, Phys. Rev. D 64, 113003.

Binetruy, P., S. Lavignac, and P. Ramond, 1996, Nucl. Phys. B 477, 353.

Boehm, F., and P. Vogel, 1987, Physics of Massive Neutrinos (Cambridge University Press, Cambridge).

Boliev, M. M., A. V. Butkevich, A. E. Chudakov, S. P. Mikheev, O. V. Suvorova, and V. N. Zakidyshev, 1999, Nucl. Phys. B, Proc. Suppl. 70, 371.

Bonn, J., et al., 2001, Nucl. Phys. B, Proc. Suppl. 91, 273.

Borodovsky, L., et al. (BNL E776 Collaboration), 1992, Phys. Rev. Lett. 68, 274.

Borzumati, F. M., Y. Grossman, E. Nardi, and Y. Nir, 1996, Phys. Lett. B 384, 123.

Borzumati, F., and Y. Nomura, 2001, Phys. Rev. D 64, 053005. Bouchez, J., et al., 1986, Z. Phys. C 32, 499.

Bugaev, E. N., and V. A. Naumov, 1989, Phys. Lett. B 232, 391. Caldwell, D. O., R. N. Mohapatra, and S. J. Yellin, 2001a, Phys. Rev. Lett. 87, 041601. 
Caldwell, D. O., R. N. Mohapatra, and S. J. Yellin, 2001b, Phys. Rev. D 64, 073001.

Carone, C. D., and M. Sher, 1998, Phys. Lett. B 420, 83.

Casas, J. A., J. R. Espinosa, A. Ibarra, and I. Navarro, 1999, Nucl. Phys. B 556, 3.

Casas, J. A., J. R. Espinosa, A. Ibarra, and I. Navarro, 2000, Nucl. Phys. B 569, 82.

Cheung, K., and O. C. Kong, 2000, Phys. Rev. D 61, 113012.

Cleveland, B. T., et al., 1998, Astrophys. J. 496, 505.

Cocco, A. G., et al. (OPERA Collaboration), 2000, Nucl. Phys. B, Proc. Suppl. 85, 125.

Cowsik, R., Yash Pal, T. N. Rengarajan, and S. N. Tandon, 1965, in Proceedings of the International Conference on Cosmic Rays, Jaipur, 1963, edited by R. R. Daniels, Vol. 6, p. 211. Creminelli, P., G. Signorelli, and A. Strumia, 2001, J. High Energy Phys. 0105, 052.

Das, A., and O. C. Kong, 1999, Phys. Lett. B 470, 149.

Davidson, S., and S. F. King, 1998, Phys. Lett. B 445, 191.

Davidson, S., and M. Losada, 2000, J. High Energy Phys. 0005, 021.

Davis, R., Jr., D. S. Harmer, and K. C. Hoffman, 1968, Phys. Rev. Lett. 20, 1205.

Daum, K., et al. (Frejus Collaboration), 1995, Z. Phys. C 66, 417.

de Bellfon, A. (for the HELLAZ Collaboration), 1999, Nucl. Phys. B, Proc. Suppl. 70, 386.

de Gouvea, A., A. Friedland, and H. Murayama, 2000, Phys. Lett. B 490, 125.

de Gouvea, A., and J. W. Valle, 2001, Phys. Lett. B 501, 115. de Holanda, P. C., and A. Yu. Smirnov, 2002, Phys. Rev. D 66, 113005.

de Rujula, A., M. B. Gavela, and P. Hernandez, 2001, Phys. Rev. D 63, 033001.

Dienes, K. R., E. Dudas, and T. Gherghetta, 1999, Nucl. Phys. B 557, 25.

Dienes, K. R., and I. Sarcevic, 2001, Phys. Lett. B 500, 133.

Dimopoulos, S., S. Raby, and F. Wilczek, 1981, Phys. Rev. D 24, 1681.

Dvali, G., and A. Yu. Smirnov, 1999, Nucl. Phys. B 563, 63.

Dydak, F., et al. (CDHS Collaboration), 1984, Phys. Lett. 134B, 281.

Dziewonski, A. M., and D. L. Anderson, 1981, Phys. Earth Planet. Inter. 25, 297.

Eguchi, K., et al. (KamLAND Collaboration), 2002, e-print hep-ex/0212021.

Ellis, J., G. K. Leontaris, S. Lola, and D. V. Nanopoulos, 1999, Eur. Phys. J. C 9, 389.

Elwood, J. K., N. Irges, and P. Ramond, 1998, Phys. Rev. Lett. 81, 5064.

Engel, R., T. K. Gaisser, and T. Stanev, 2000, Phys. Lett. B 472, 113.

Ermilova, V. K., V. A. Tsarev, and V. A. Chechin, 1986, Kratk. Soobshch. Fiz. 5, 26.

Eskut, E., et al. (CHORUS Collaboration), 2001, Phys. Lett. B 497, 8.

Eyal, G., and Y. Nir, 1999, J. High Energy Phys. 9906, 024.

Faid, B., G. L. Fogli, E. Lisi, and D. Montanino, 1997, Phys. Rev. D 55, 1353.

Faraggi, A. E., and M. Pospelov, 1999, Phys. Lett. B 458, 237. Farzan, Y., O. L. Peres, and A. Yu. Smirnov, 2001, Nucl. Phys. B 612, 59 .

Fiorentini, G., V. A. Naumov, and F. L. Villante, 2001, Phys. Lett. B 510, 173.
Fogli, G. L., and E. Lisi, 1995a, Astropart. Phys. 3, 185.

Fogli, G. L., and E. Lisi, 1995b, Phys. Rev. D 52, 2775.

Fogli, G. L., E. Lisi, and A. Marrone, 2001, Phys. Rev. D 64, 093005.

Fogli, G. L., E. Lisi, A. Marrone, D. Montanino, and A. Palazzo, 2002, Phys. Rev. D 66, 053010.

Fogli, G. L., E. Lisi, A. Marrone, and G. Scioscia, 1999a, e-print hep-ph/9906450

Fogli, G. L., E. Lisi, A. Marrone, and G. Scioscia, 1999b, Phys. Rev. D 59, 033001.

Fogli, G. L., E. Lisi, and D. Montanino, 1994, Phys. Rev. D 49, 3626.

Fogli, G. L., E. Lisi, and D. Montanino, 1995, Astropart. Phys. 4, 177.

Fogli, G. L., E. Lisi, and D. Montanino, 1996, Phys. Rev. D 54, 2048.

Fogli, G. L., E. Lisi, and D. Montanino, 1998, Astropart. Phys. 9, 119.

Fogli, G. L., E. Lisi, D. Montanino, and A. Palazzo, 2000a, Phys. Rev. D 62, 013002.

Fogli, G. L., E. Lisi, D. Montanino, and A. Palazzo, 2000b, Phys. Rev. D 62, 113004.

Fogli, G. L., E. Lisi, D. Montanino, and A. Palazzo, 2001, Phys. Rev. D 64, 093007.

Fogli, G. L., E. Lisi, D. Montanino, and G. Scioscia, 1997, Phys. Rev. D 55, 4385.

Fogli, G. L., and G. Nardulli, 1979, Nucl. Phys. B 160, 116.

Fogli, G. L., et al., 2002, e-print hep-ph/0212127.

Foot, R., R. R. Volkas, and O. Yasuda, 1998, Phys. Rev. D 58, 013006.

Frampton, P. H., and S. L. Glashow, 1999, Phys. Lett. B 461, 95.

Friedland, A., 2000, Phys. Rev. Lett. 85, 936.

Friedland, A., 2001, Phys. Rev. D 64, 013008.

Fritzsch, H., and Z. Xing, 1996, Phys. Lett. B 372, 265.

Fritzsch, H., and Z. Xing, 2000, Phys. Rev. D 61, 073016.

Froggatt, C. D., and H. B. Nielsen, 1979, Nucl. Phys. B 147, 277.

Fukuda, S., et al. (SuperKamiokande Collaboration), 2000, Phys. Rev. Lett. 85, 3999.

Fukuda, S., et al. (SuperKamiokande Collaboration), 2001, Phys. Rev. Lett. 86, 5651.

Fukuda, Y., et al. (Kamiokande Collaboration), 1994, Phys. Lett. B 335, 237.

Fukuda, Y., et al. (Kamiokande Collaboration), 1996, Phys. Rev. Lett. 77, 1683.

Fukuda, Y., et al. (SuperKamiokande Collaboration), 1998, Phys. Rev. Lett. 81, 1562.

Fukuda, Y., et al. (SuperKamiokande Collaboration), 1999a, Phys. Lett. B 467, 185.

Fukuda, Y., et al. (SuperKamiokande Collaboration), 1999b, Phys. Rev. Lett. 82, 5194.

Fukugita, M., M. Tanimoto, and T. Yanagida, 1998, Phys. Rev. D 57, 4429.

Fukugita, M., M. Tanimoto, and T. Yanagida, 1999, Phys. Rev. D 59, 113016.

Gago, A. M., H. Nunokawa, and R. Zukanovich Funchal, 2001, Phys. Rev. D 63, 013005.

Gaisser, T. K., M. Honda, K. Kasahara, H. S. Lee, S. Midorikawa, V. Naumov, and T. Stanev, 1996, Phys. Rev. D 54, 5578.

Gaisser, T. K., and T. Stanev, 1998, Phys. Rev. D 57, 1977.

Gaisser, T. K., T. Stanev, and G. Barr, 1988, Phys. Rev. D 38, 85. 
Garzelli, M. V., and C. Giunti, 2001, J. High Energy Phys. 0112, 017.

Gell-Mann, M., P. Ramond, and R. Slansky, 1979, in Supergravity, edited by P. van Nieuwenhuizen and D. Z. Freedman (North-Holland, Amsterdam), p. 315.

Gemmeke, H., et al., 1990, Nucl. Instrum. Methods Phys. Res. A 289, 490.

Gonzalez-Garcia, M. C., P. C. de Holanda, C. Peña-Garay, and J. W. F. Valle, 2000, Nucl. Phys. B 573, 3.

Gonzalez-Garcia, M. C., N. Fornengo, and J. W. F. Valle, 2000, Nucl. Phys. B 580, 58.

Gonzalez-Garcia, M. C., and M. Maltoni, 2002, Eur. Phys. J C 26, 417.

Gonzalez-Garcia, M. C., M. Maltoni, C. Peña-Garay, and J. W. F. Valle, 2001, Phys. Rev. D 63, 033005.

Gonzalez-Garcia, M. C., H. Nunokawa, O. L. G. Peres, T. Stanev, and J. W. F. Valle, 1998, Phys. Rev. D 58, 033004.

Gonzalez-Garcia, M. C., H. Nunokawa, O. L. G. Peres, and J. W. F. Valle, 1999, Nucl. Phys. B 543, 3.

Gonzalez-Garcia, M. C., and C. Peña-Garay, 2000, Phys. Rev. D 62, 031301.

Gonzalez-Garcia, M. C., and C. Peña-Garay, 2001, Phys. Rev. D 63, 073013.

Gonzalez-Garcia, M. C., C. Peña-Garay, Y. Nir, and A. Yu. Smirnov, 2001, Phys. Rev. D 63, 013007.

Gonzalez-Garcia, M. C., C. Peña-Garay, and A. Yu. Smirnov, 2001, Phys. Rev. D 63, 113004.

Groom, D. E., et al. (Particle Data Group), 2000, Eur. Phys. J. C 15, 1 .

Grossman, Y., and M. Neubert, 2000, Phys. Lett. B 474, 361.

Grossman, Y., and Y. Nir, 1995, Nucl. Phys. B 448, 30.

Grossman, Y., Y. Nir, and Y. Shadmi, 1998, J. High Energy Phys. 9810, 007.

Haba, N., and H. Murayama, 2001, Phys. Rev. D 63, 053010. Hagiwara, K., and N. Okamura, 1999, Nucl. Phys. B 548, 60.

Hall, L., and H. Murayama, 1998, Phys. Lett. B 436, 323.

Hall, L., H. Murayama, and N. Weiner, 2000, Phys. Rev. Lett. 84, 2572.

Halprin, A., 1986, Phys. Rev. D 34, 3462.

Hampel, W., et al. (GALLEX Collaboration), 1999, Phys. Lett. B 447, 127.

Harvey, J. A., D. B. Reiss, and P. Ramond, 1982, Nucl. Phys. B 199, 223.

Hata, N., S. Bludman, and P. Langacker, 1994, Phys. Rev. D 49, 3622 .

Hata, N., and P. Langacker, 1997, Phys. Rev. D 56, 6107.

Haxton, W. C., 1986, Phys. Rev. Lett. 57, 1271.

Heeger, K. M., and R. G. H. Robertson, 1996, Phys. Rev. Lett. 77, 3720 .

Hidaka, K., M. Honda, and S. Midorikawa, 1988, Phys. Rev. Lett. 61, 1537.

Hirata, S. H., et al. (Kamiokande Collaboration), 1992, Phys. Lett. B 280, 146.

Hirsch, M., and S. F. King, 2001, Phys. Lett. B 516, 103.

Honda, M., T. Kajita, K. Kasahara, and S. Midorikawa, 1996, Prog. Theor. Phys. Suppl. 123, 483.

Honda, M., T. Kajita, S. Midorikawa, and K. Kasahara, 1995, Phys. Rev. D 52, 4985.

Honda, M., K. Kasahara, K. Hidaka, and S. Midorikawa, 1990, Phys. Lett. B 248, 193.

Huber, S. J., and Q. Shafi, 2001, Phys. Lett. B 512, 365.

Ibanez, L. E., and G. G. Ross, 1981, Phys. Lett. B 105, 439.

Ioannisian, A., and A. Pilaftsis, 2000, Phys. Rev. D 62, 066001.
Irges, N., S. Lavignac, and P. Ramond, 1998, Phys. Rev. D 58, 035003.

Joshipura, A. S., and S. D. Rindani, 2000a, Eur. Phys. J. C 14, 85.

Joshipura, A. S., and S. D. Rindani, 2000b, Phys. Lett. B 494, 114.

Kajita, T., and Y. Totsuka, 2001, Rev. Mod. Phys. 73, 85.

Kayser, B., F. Gibrat-Debu, and F. Perrier, 1989, The Physics of Massive Neutrinos, World Scientific Lecture Notes in Physics No. 25 (World Scientific, Singapore), p. 1.

Kim, C. W. and A. Pevsner, 1993, Neutrinos in Physics and Astrophysics, Contemporary Concepts in Physics, No. 8 (Harwood Academic, Chur, Switzerland).

King, S. F., 1998, Phys. Lett. B 439, 350.

King, S. F., 1999, Nucl. Phys. B 562, 57.

King, S. F., 2000, Nucl. Phys. B 576, 85.

Kirsten, T. (GNO Collaboration), 2002, Talk given at $\nu$-2002 conference, Munich, May 2002.

Klapdor-Kleingrothaus, H. V., H. Pas, and A. Yu. Smirnov, 2001, Phys. Rev. D 63, 073005.

Klapdor-Kleingrothaus, H. V., et al., 2001, Eur. Phys. J. A 12, 147.

Kobayashi, M., and T. Maskawa, 1973, Prog. Theor. Phys. 49, 652.

Krastev, P. I., and S. T. Petcov, 1988, Phys. Lett. B 207, 64.

Kuo, T. K., and J. Pantaleone, 1986, Phys. Rev. Lett. 57, 1805.

Kuo, T. K., and J. Pantaleone, 1989, Rev. Mod. Phys. 61, 937.

Landau, L., 1932, Phys. Z. Sowjetunion 2, 46.

Lande, K., et al., 1999, Nucl. Phys. B, Proc. Suppl. 77, 13.

Langacker, P., S. T. Petcov, G. Steigman, and S. Toshev, 1987, Nucl. Phys. B 282, 589.

Langacker, P., and N. Polonsky, 1995, Phys. Rev. D 52, 3081.

Lanou, R. E., et al., 1993, J. Low Temp. Phys. 93, 785.

Learned, J. G., S. Pakvasa, and T. J. Weiler, 1988, Phys. Lett. B 207, 79 .

Lee, H., and Y. S. Koh, 1990, Nuovo Cimento Soc. Ital. Fis., B 105, 883.

Leurer, M., Y. Nir, and N. Seiberg, 1993, Nucl. Phys. B 398, 319.

Leurer, M., Y. Nir, and N. Seiberg, 1994, Nucl. Phys. B 420, 468.

Lipari, P., 2000, Astropart. Phys. 14, 153.

Lipari, P., T. K. Gaisser, and T. Stanev, 1998, Phys. Rev. D 58, 073003.

Lipari, P., and M. Lusignoli, 1998, Phys. Rev. D 58, 073005.

Lipkin, H. J., 1999, e-print hep-ph/9901399.

Lisi, E., A. Marrone, D. Montanino, A. Palazzo, and S. T. Petcov, 2001, Phys. Rev. D 63, 093002.

Lobashev, V. M., et al., 2001, Nucl. Phys. B, Proc. Suppl. 91, 280.

Lohmann, W., R. Kopp, and R. Voss, 1985, CERN Yellow Report EP/85-03.

Lukas, A., P. Ramond, A. Romanino, and G. G. Ross, 2000, Phys. Lett. B 495, 136.

Lukas, A., P. Ramond, A. Romanino, and G. G. Ross, 2001, J. High Energy Phys. 0104, 010.

Ma, E., M. Raidal, and U. Sarkar, 2000, Phys. Rev. Lett. 85, 3769.

Ma, E., and G. Rajasekaran, 2001, Phys. Rev. D 64, 113012.

Maekawa, N., 2001, Prog. Theor. Phys. 106, 401.

Maki, Z., M. Nakagawa, and S. Sakata, 1962, Prog. Theor. Phys. 28, 870.

Mannheim, P. D., 1988, Phys. Rev. D 37, 1935. 
Maris, M., and S. T. Petcov, 1997, Phys. Rev. D 56, 7444.

Marquet, C., et al. (NEMO Collaboration), 2000, Nucl. Phys. B, Proc. Suppl. 87, 298.

McDonald, A. B., et al. (SNO Collaboration), 2000, Nucl. Phys. B, Proc. Suppl. 91, 21.

McFarland, K. S., et al. (CCFR Collaboration), 1995, Phys. Rev. Lett. 75, 3993.

Mikheyev, S. P., and A. Yu. Smirnov, 1985, Yad. Fiz. 42, 1441 [Sov. J. Nucl. Phys. 42, 913 (1985)].

Mikheyev, S. P., and A. Yu. Smirnov, 1987, Sov. Phys. Usp. 30, 759.

Mohapatra, R. N., S. Nandi, and A. Perez-Lorenzana, 1999, Phys. Lett. B 466, 115.

Mohapatra, R. N., and A. Perez-Lorenzana, 2000, Nucl. Phys. B 576, 466.

Mohapatra, R. N., A. Perez-Lorenzana, and C. A. de Sousa Pires, 2000a, Phys. Lett. B 474, 355.

Mohapatra, R. N., A. Perez-Lorenzana, and C. A. de Sousa Pires, 2000b, Phys. Lett. B 491, 143.

Mohapatra, R. N., and G. Senjanovic, 1980, Phys. Rev. Lett. 44, 912.

Murayama, H., and T. Yanagida, 2001, Phys. Lett. B 520, 263.

Nakahata, M., et al., 1986, J. Phys. Soc. Jpn. 55, 3786.

Nakamura, S., T. Sato, V. Gudkov, and K. Kubodera, 2001, Phys. Rev. C 63, 034617.

Naples, D., et al. (CCFR Collaboration), 1999, Phys. Rev. D 59, 031101.

Nir, Y., 2000, J. High Energy Phys. 0006, 039.

Nir, Y., and Y. Shadmi, 1999, J. High Energy Phys. 9905, 023. Nishikawa, K., et al., 1997, KEK-PS proposal, Nucl. Phys. B, Proc. Suppl. 59, 289.

Nishikawa, K., et al. (K2K Collaboration), 2001, J. High Energy Phys., Conference Proceedings, PRHEP-hep2001/298.

Oberauer, L., 1999, Nucl. Phys. B, Proc. Suppl. 77, 48.

Ortiz, C. E., A. Garcia, R. A. Waltz, M. Bhattacharya, and A.

K. Komives, 2000, Phys. Rev. Lett. 85, 2909.

Osborne, J. L., S. S. Said, and A. W. Wolfendale, 1965, Proc. Phys. Soc. London 86, 93.

Pakvasa, S., and J. Pantaleone, 1990, Phys. Rev. Lett. 65, 2479.

Pantaleone, J., 1990, Phys. Lett. B 251, 618.

Parke, S. J., 1986, Phys. Rev. Lett. 57, 1275.

Parke, S. J., 1995, Phys. Rev. Lett. 74, 839.

Perez, G., 2000, J. High Energy Phys. 0012, 027.

Petcov, S. T., 1987, Phys. Lett. B 191, 299.

Petcov, S. T., 1988a, Phys. Lett. B 200, 373.

Petcov, S. T., 1988b, Phys. Lett. B 214, 139.

Petcov, S. T., 1998, Phys. Lett. B 434, 321.

Petcov, S. T., and J. Rich, 1989, Phys. Lett. B 224, 426.

Piepke, A., et al., 2001, Nucl. Phys. B, Proc. Suppl. 91, 99.

Pontecorvo, B., 1957, Zh. Eksp. Teor. Fiz. 33, 549 [Sov. Phys. JETP 6, 429 (1958)].

Raghavan, R. S., 1997, Phys. Rev. Lett. 78, 3618.

Ramond, P., 1979, preprint CALT-68-709, Invited talk given at Sanibel Symposium, hep-ph/9809459.

Ramond, P., 1999, Journeys Beyond The Standard Model (Perseus, Reading, MA).

Randall, L., and R. Sundrum, 1999, Phys. Rev. Lett. 83, 3370.

Reines, F., M. F. Crouch, T. L. Jenkins, W. R. Kropp, H. S. Gurr, G. R. Smith, J. P. F. Sellschop, and B. Meyer, 1965, Phys. Rev. Lett. 15, 429.
Romosan, C., et al. (CCFR Collaboration), 1997, Phys. Rev. Lett. 78, 2912.

Sato, J., and T. Yanagida, 1998, Phys. Lett. B 430, 127.

Sato, J., and T. Yanagida, 2000, Phys. Lett. B 493, 356.

Schechter, J., and J. W. F. Valle, 1980a, Phys. Rev. D 21, 309.

Schechter, J., and J. W. F. Valle, 1980b, Phys. Rev. D 22, 2227.

Schechter, J., and J. W. F. Valle, 1981, Phys. Rev. D 24, 1883.

Shafi, Q., and Z. Tavartkiladze, 1999, Phys. Lett. B 451, 129.

Shafi, Q., and Z. Tavartkiladze, 2000a, Phys. Lett. B 473, 272.

Shafi, Q., and Z. Tavartkiladze, 2000b, Phys. Lett. B 487, 145.

Shafi, Q., and Z. Tavartkiladze, 2000c, Nucl. Phys. B 573, 40.

Shafi, Q., and Z. Tavartkiladze, 2000d, Phys. Lett. B 482, 145.

Shi, X., and D. N. Schramm, 1992, Phys. Lett. B 283, 305.

Shibuya, H., et al., 1997, CERN-SPSC-97-24.

Shrock, R., 1980, Phys. Lett. B 96, 159.

Simpson, J. A., 1983, Annu. Rev. Nucl. Part. Sci. 33, 323.

Smith, C. L., 1972, Phys. Rep. 3, 261.

Smy, M., 2002, Talk given at $\nu$-2002 conference, Munich, May 2002.

Tanimoto, M., 1999a, Phys. Rev. D 59, 017304.

Tanimoto, M., 1999b, Phys. Lett. B 456, 220.

Tanimoto, M., 2000, Phys. Lett. B 483, 417.

Tanimoto, M. T., T. Watari, and T. Yanagida, 1999, Phys. Lett. B 461, 345.

Teshima, T., and T. Sakai, 1999, Prog. Theor. Phys. 101, 147.

Toshito, T., et al., 2001, e-print hep-ex/0105023.

Tserkovnyak, Y., R. Komar, C. Nally, and C. Waltham, 2003, Astropart. Phys. 18, 449.

Turck-Chieze, S., S. Cahen, M. Casse, and C. Doom, 1988, Astrophys. J. 335, 415.

Ushida, N., et al. (BNL E531 Collaboration), 1986, Phys. Rev. Lett. 57, 2897.

Valuev, V., et al. (NOMAD Collaboration), 2001, J. High Energy Phys., Conference Proceedings, PRHEP-hep2001/190.

Vidyakin, G. S., et al., 1994, JETP Lett. 59, 390.

Villante, F. L., G. Fiorentini, and E. Lisi, 1999, Phys. Rev. D 59, 013006.

Vissani, F., 1999, J. High Energy Phys. 9906, 022.

Vissani, F., 1998, J. High Energy Phys. 9811, 025.

Vissani, F., 2001a, Nucl. Phys. B, Proc. Suppl. 100, 273.

Vissani, F., 2001b, Phys. Lett. B 508, 79.

Vissani, F., and A. Yu. Smirnov, 1998, Phys. Lett. B 432, 376.

Wetterich, C., 1999, Phys. Lett. B 451, 397.

Wilczek, F., 2001, Int. J. Mod. Phys. A 16, 1653.

Wojcicki, S. G., 2001, Nucl. Phys. B, Proc. Suppl. 91, 216.

Wolf, J., et al. (KARMEN Collaboration), 2001, J. High Energy Phys., Conference Proceedings, PRHEP-hep2001/301.

Wolfenstein, L., 1978, Phys. Rev. D 17, 2369.

Wolfenstein, L., 1981, Phys. Lett. 107B, 77.

Wu, Y., 1999a, Phys. Rev. D 60, 073010.

Wu, Y., 1999b, Eur. Phys. J. C 10, 491.

Wu, Y., 1999c, Int. J. Mod. Phys. A 14, 4313.

Yanagida, T., 1979, in Proceedings of Workshop on Unified Theory and Baryon Number in the Universe, edited by $\mathrm{O}$. Sawada and A. Sugamoto, KEK Report KEK-97-18, p. 315. Yasuda, O., 1998, Phys. Rev. D 58, 091301.

Zacek, G., et al., 1986, Phys. Rev. D 34, 2621.

Zatsepin, G. T., and V. A. Kuzmin, 1962, Sov. J. Nucl. Phys. 14, 1294.

Zener, C., 1932, Proc. R. Soc. London, Ser. A 137, 696. 